\title{
SNARE-mediated membrane fusion on pore-spanning membranes - several fusion pathways analyzed by single-vesicle content release
}

\section{Dissertation}

for the award of the degree

\section{Doctor rerum naturalium}

of the Georg-August-Universität Göttingen

within the doctoral program

Physics of Biological and Complex Systems

of the Georg-August University School of Science (GAUSS)

submitted by

Peter Mühlenbrock

from Göttingen

Göttingen 2020 



\section{SNARE-mediated membrane fusion on pore-spanning membranes - several fusion pathways analyzed by single-vesicle content release}

\section{Dissertation}

for the award of the degree

\section{Doctor rerum naturalium}

of the Georg-August-Universität Göttingen

within the doctoral program

Physics of Biological and Complex Systems

of the Georg-August University School of Science (GAUSS)

submitted by

Peter Mühlenbrock

from Göttingen

Göttingen 2020 



\section{Members of the thesis advisory committee}

Prof. Dr. Claudia Steinem

Institut für Organische und Biomolekulare Chemie

Georg-August-Universität Göttingen

Prof. Dr. Tim Salditt

Institut für Röntgenphysik

Georg-August-Universität Göttingen

Prof. Dr. Silvio O. Rizzoli

Abteilung für Neuro- und Sinnesphysiologie

Universitätsmedizin Göttingen

\section{Members of the examination board}

First Referee: Prof. Dr. Claudia Steinem

Institut für Organische und Biomolekulare Chemie

Georg-August-Universität Göttingen

Second Referee: Prof. Dr. Tim Salditt

Institut für Röntgenphysik

Georg-August-Universität Göttingen

Prof. Dr. Silvio O. Rizzoli

Abteilung für Neuro- und Sinnesphysiologie

Universitätsmedizin Göttingen

Prof. Dr. Michael Meinecke

Institut für Zelluläre Biochemie

Universitätsmedizin Göttingen

Prof. Dr. Reinhard Jahn

Abteilung für Neurobiologie

Max-Planck-Institut für Biophysikalische Chemie Göttingen

Prof. Dr. Jörg Enderlein

Drittes Physikalisches Institut

Georg-August-Universität Göttingen

\section{Date of oral Examination}

18.12 .2020 



\section{Declaration}

I, Peter Mühlenbrock, hereby certify that my doctoral thesis entitled "SNARE-mediated membrane fusion on pore-spanning membranes - several fusion pathways analyzed by single-vesicle content release" has been written independently and with no other sources or aids than quoted.

Göttingen, 2020

Peter Mühlenbrock 



\section{Contents}

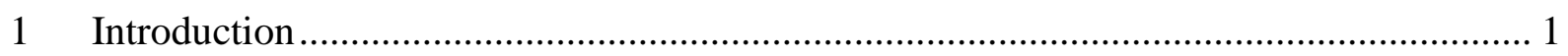

1.1 SNARE-mediated membrane fusion ............................................................... 1

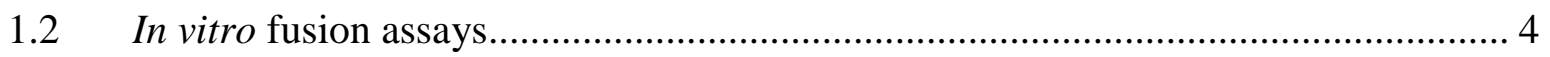

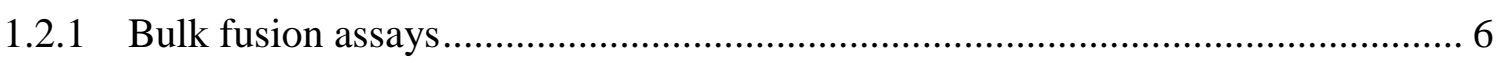

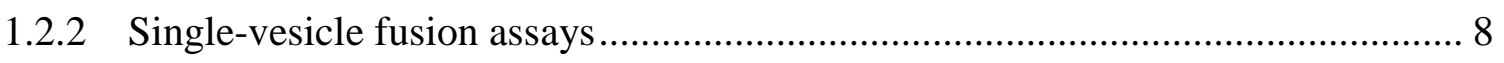

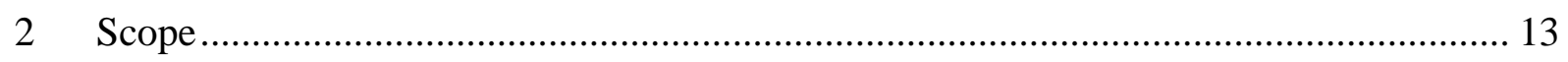

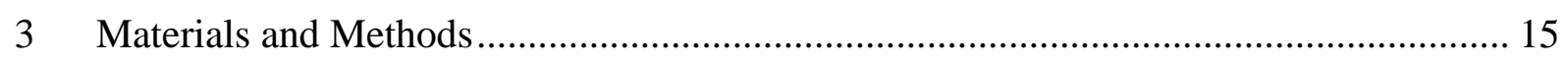

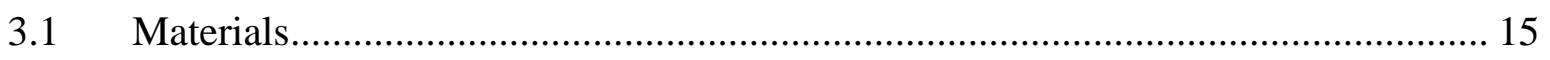

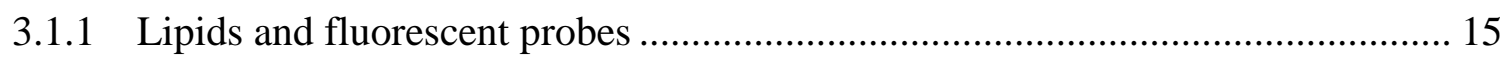

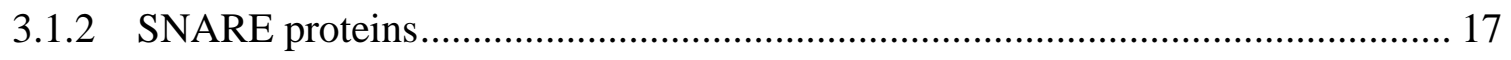

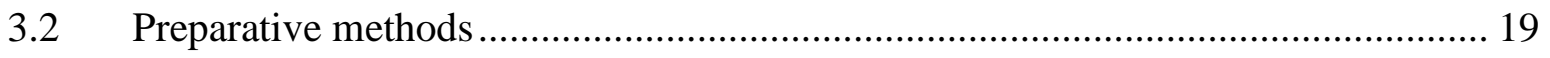

3.2.1 SDS-polyacrylamide gel electrophoresis ................................................. 19

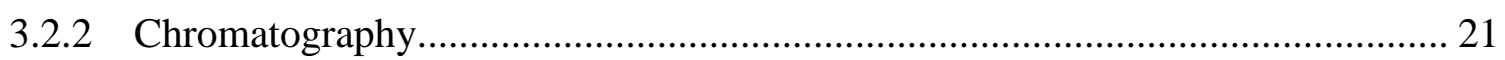

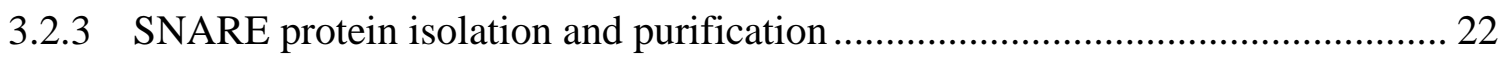

3.2.4 SNARE protein reconstitution into liposomes ............................................... 35

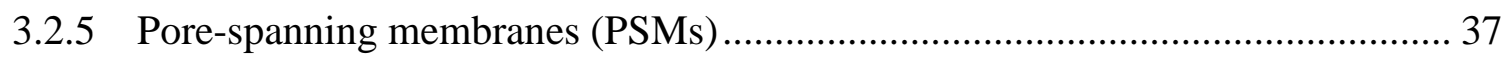

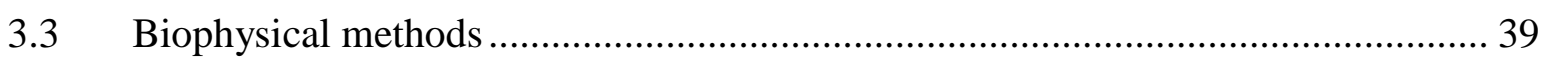

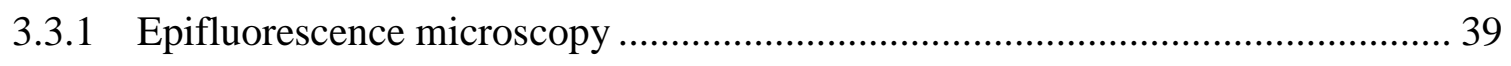

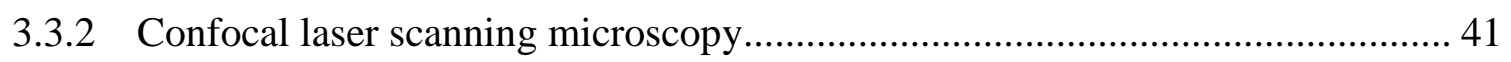

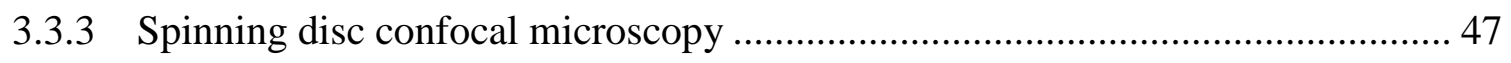

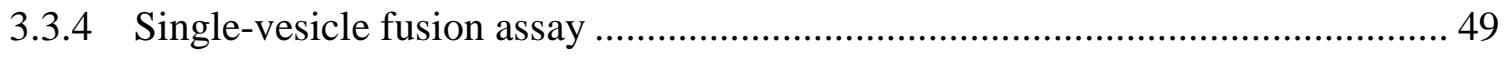

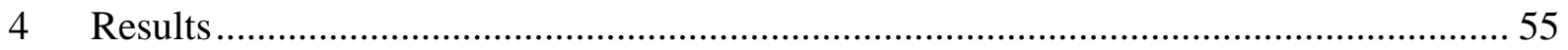

4.1 Functional reconstitution of SNAREs into liposomes ...................................... 56

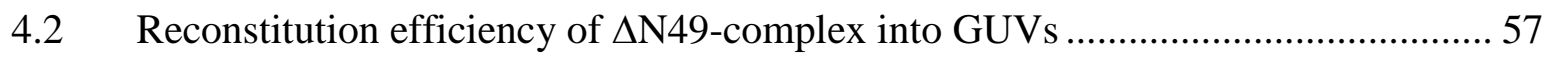

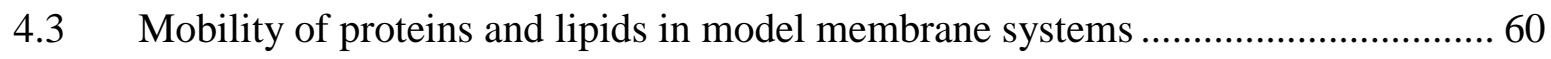

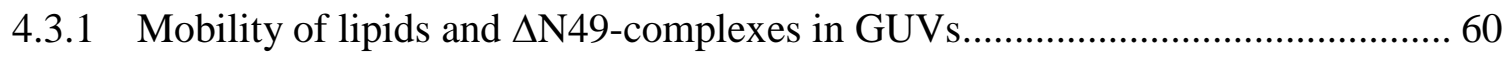


4.3.2 Mobility of lipids and $\Delta$ N49-complexes in PSMs

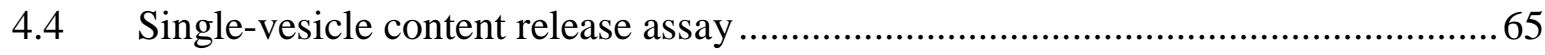

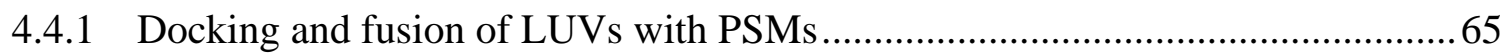

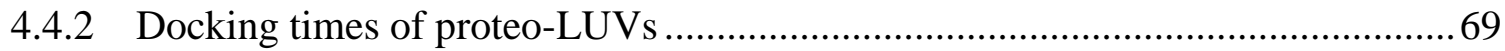

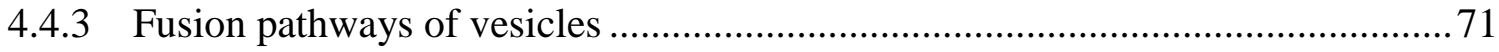

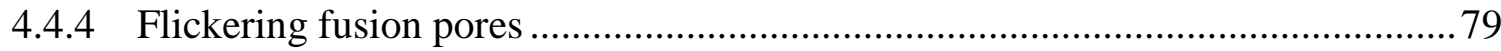

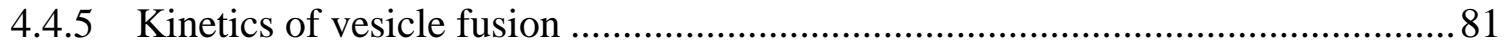

4.4.6 Docking and fusion of vesicles with the f-PSM .............................................. 86

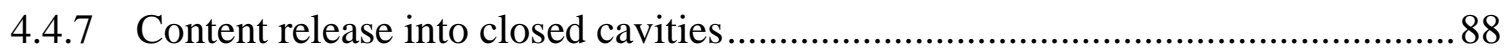

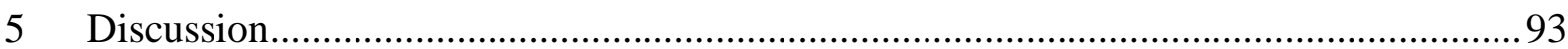

5.1 Reconstitution efficiency of SNAREs into GUVs ............................................ 93

5.2 Mobility of proteins and lipids in model membrane systems..............................95

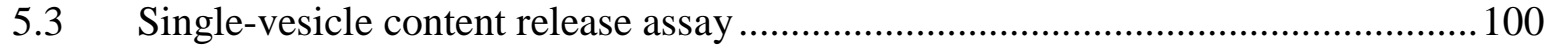

5.3.1 SNARE-mediated docking and fusion of LUVs with PSMs.............................100

5.3.2 Docking times and fusion efficiencies of proteo-LUVs .................................. 107

5.3.3 Pathways and kinetics of vesicle fusion ......................................................... 111

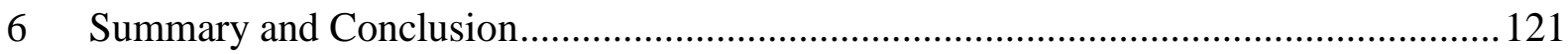

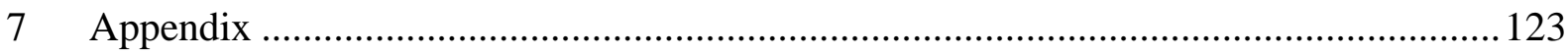

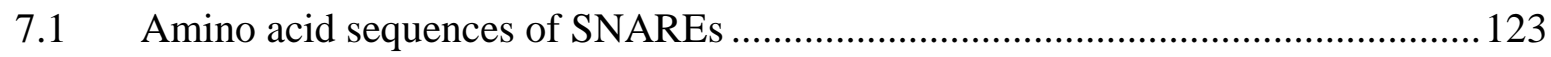

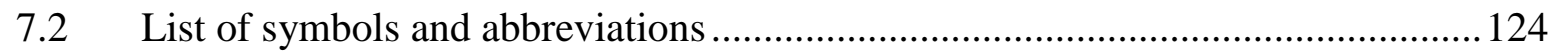

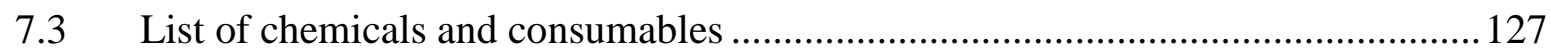

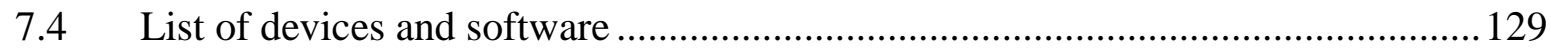

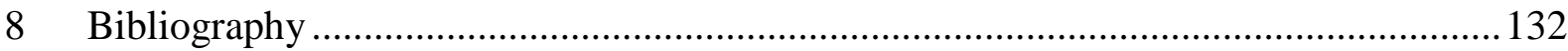


Abstract. The fusion of neurotransmitter filled vesicles with the presynaptic membrane is the key step in the neuronal signaling cascade and is mediated by soluble $N$-ethylmaleimidesensitive factor attachment receptor proteins (SNAREs). The interaction of the three SNARE proteins synaptobrevin 2 (syb 2), syntaxin 1A, and SNAP25 (synaptosomal associated protein of $25 \mathrm{kDa}$ ) is pivotal to overcome the energy barrier that leads to merging of the opposing lipid bilayers and results in the transfer of neurotransmitters across the presynaptic membrane and into the synaptic cleft.

To investigate this fundamental process, pore-spanning membranes (PSMs) were utilized in this work as a model system of the presynaptic membrane. PSMs are continuous lipid bilayers with solid supported parts (s-PSM) as well as freestanding membranes spanning large aqueous compartments (f-PSM). Thus they are suitable to monitor the process of content transfer through a fusion pore of added vesicles filled with a water soluble dye by means of fluorescence microscopy. Simultaneous imaging of lipid dye diffusion from the PSM into the vesicular membrane via a fusion stalk was used to quantify different fusion pathways.

The surface of porous substrates with pore diameters of $1.2 \mu \mathrm{m}$ or $3.5 \mu \mathrm{m}$ was hydrophilized with a self-assembled monolayer of 6-mercapto-1-hexanol formed on gold and PSMs were then produced by spreading of acceptor complex containing giant unilamellar vesicles. Differences in densities of target SNAREs inside PSMs were noticeable in variations of docking efficiencies of syb 2 containing, sulforhodamine B (SRB) filled liposomes to the PSMs. The fusion pore formation was then directly visualized by imaging the transfer of SRB from inside the vesicle into the space underneath the f-PSM. This process proved to be very rapid and distinguishable from rarely occurring burst events. Furthermore, it mainly occurred simultaneously with lipid diffusion from the PSM into the vesicular membrane and resulted predominantly in a full release of vesicular content. Additionally, this main fusion pathway was more likely for smaller vesicles and included a rapid and full collapse of the fusing vesicle into the PSM and thus shows distinct features of full-collapse fusion observed in vivo. Apart from this, the premature closing of the fusion pore could lead to a stable three-dimensional postfusion structure that was often times accompanied by a partial SRB release. From this state the fusion pore could open again leading to the complex fusion behavior of a flickering fusion pore. In summary, the results of this study show that the diverse fusion pathways observed in vivo likely are an intrinsic property of the minimal fusion machinery and not caused by the interplay of additional proteins. 
Zusammenfassung. Die Fusion von Neurotransmitter gefüllten Vesikeln mit der präsynaptischen Membran ist der Schlüsselschritt in der neuronalen Signaltransduktion und wird durch SNARE- (soluble N-ethylmaleimide-sensitive factor attachment receptor) Proteine vermittelt. Die Interaktion der drei SNARE-Proteine Synaptobrevin 2, Syntaxin 1A und SNAP25 (synaptosomal associated protein of $25 \mathrm{kDa}$ ) überwindet die Energiebarriere der Verschmelzung beider Lipid Doppelschichten, was zum Transfer von Neurotransmittern über die präsynaptische Membran und in den synaptischen Spalt führt.

Um diesen essenziellen Schritt zu untersuchen, wurden in dieser Arbeit porenüberspannende Membranen (PSMs) als Modellsystem der präsynaptischen Membran genutzt. PSMs sind Lipiddoppelschichten, die aus festkörperunterstützten (s-PSM) und porenüberspannenden, freitragenden Bereichen (f-PSM) bestehen. Durch Letztere sind sie geeignet, den Transferprozess von wasserlöslichen Fluorophoren durch eine Fusionspore aus dem Inneren von hinzugegebenen Vesikeln über die PSM mittels Fluoreszenzmikroskopie zu beobachten. Durch die gleichzeitige Detektion der Lipiddiffusion von der PSM in den Vesikel wurden verschiedene Fusionswege quantifiziert.

Die Oberfläche von porösen Substraten mit Porendurchmessern von $1.2 \mu$ m oder $3.5 \mu \mathrm{m}$ wurde hydrophilisiert, indem eine selbstorganisierte Monoschicht aus 6-Mercapto-1-hexanol auf Gold gebildet wurde. PSMs wurden dann durch das Spreiten von target-SNARE enthaltenden riesigen unilamellaren Vesikeln erzeugt. Unterschiede in der Konzentration der t-SNAREs in PSMs waren als Schwankungen der Dockingeffizienz von syb 2 enthaltenden Liposomen an die PSMs sichtbar. Anschließend wurde die Ausbildung einer Fusionspore durch den Ausstrom von Sulforhodamin B (SRB) vom Vesikelinnern in die Kompartimente unterhalb der f-PSM beobachtet. Dieser Prozess geschah quasi simultan mit der Lipidvermischung der beiden Membranen und führte bevorzugt zu einer vollständigen Ausschüttung des Vesikelinhalts. Zudem war dieser Hauptfusionsweg wahrscheinlicher für kleinere Vesikel und endete in einem raschen, vollständigen Kollaps des fusionierten Vesikels in die PSM und zeigt somit charakteristische Eigenschaften der full-collapse Fusion, welche in vivo beobachtbar ist. Abgesehen davon konnte das vorzeitige Schließen der Fusionspore zu einem unvollständigen Ausstrom von SRB und einer stabilen, dreidimensionalen Postfusionsstruktur führen. Aus diesem Zustand konnte der Vesikel erneut fusionieren, was zum komplexen Fusionsweg einer flackernden Fusionspore führte. Die Komplexität des Fusionsprozesses, wie er in vivo beobachtet wird, scheint somit nicht durch die Vielzahl an Proteinen, sondern bereits durch die minimale Fusionsmaschinerie herbeigeführt zu werden. 


\section{Introduction}

Membranes - two-dimensional bilayers consisting of lipids, proteins, and other molecules - are one of the key building blocks in various different life forms and are impermeable for most larger molecules and ions. They separate the interior of cells from the surrounding media and form highly structured compartments inside the cell where different metabolic reactions can take place simultaneously. The controlled exchange of small molecules and ions with the cell exterior and their transport between the compartments is mediated by ion channels and carrier proteins embedded inside the membrane. In contrast to that, larger molecules are filled into vesicles that are released from the host membrane via a process called budding and deliver the cargo by fusing with the target membrane. These two processes need to be highly regulated in order to prevent the fusion of liposomes with the wrong compartment and thus interfering with its functionality.

\subsection{SNARE-mediated membrane fusion}

A special case - the fusion of vesicles with the plasma membrane - is also referred to as exocytosis and can be found at the presynaptic membrane of synaptic boutons. Here, neurotransmitter filled vesicles fuse with the target membrane to release their cargo into the synaptic cleft. However, at the resting state membranes are $~ 10-20 \mathrm{~nm}$ apart from each other, a distance that has to be overcome for fusion to occur. This renders the process highly endergonic, as the reduction to a distance of few nanometers is e.g. concomitant with the dehydration of the lipid head groups. This raises the question, what drives the fusion of synaptic vesicles and how is it controlled?

The way for identifying the key players of membrane fusion was cleared by Rothman and coworkers by isolating the $N$-ethylmaleimide-sensitive factor (NSF) due to its ability to restore vesicle transport after deactivation with $N$-ethylmaleimide (NEM) and identifying its role in the fusion process. ${ }^{[1,2]}$ Further studies showed that NSF binds to the target membrane via the soluble NSF attachment protein (SNAP) and that functional transport requires SNAP activity. ${ }^{[3]}$ This led to the discovery of the receptors for SNAP in synapses called soluble $N$-ethylmaleimidesensitive factor attachment receptor proteins (SNAREs): syntaxin 1A (syx 1A), SNAP25 (synaptosomal associated protein of $25 \mathrm{kDa}$ ), and synaptobrevin 2 (syb 2). ${ }^{[4]}$ These proteins turned out to catalyze the process of neurotransmitter release at the presynaptic membrane together with different regulatory proteins, such as muncs, complexin, and synaptotagmin 
(Figure 1.1). ${ }^{[5,6]}$ Briefly, vesicles are docked and primed in the active zone, also called readily releasable pool, the arriving of an action potential from the axon then triggers the opening of $\mathrm{Ca}^{2+}$ channels at the presynaptic membrane, and the influx of $\mathrm{Ca}^{2+}$ in turn stimulates the release of neurotransmitters into the synaptic cleft. ${ }^{[7,8]}$ While this process is highly regulated by different proteins, early studies revealed that only the aforementioned three SNARE proteins are needed to drive membrane fusion. ${ }^{[9,10]}$

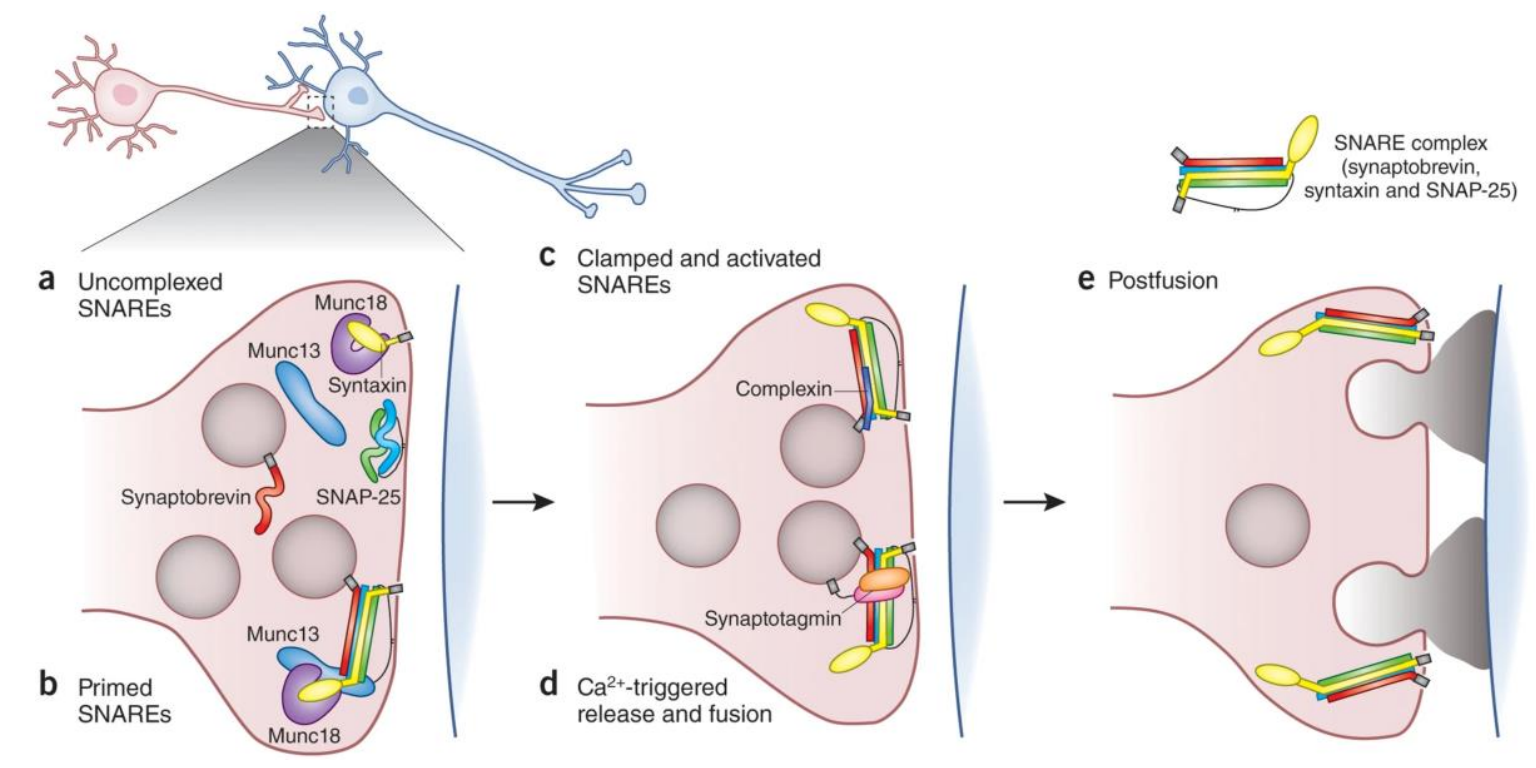

Figure 1.1 Schematic illustration of the process of exocytosis at synapses. (a,b) Priming of SNAREs through Munc 13 and 18 close to the presynaptic membrane. (c,d) SNAREs do not undergo fusion yet but are held in place by presumably complexin and synaptotagmin, until the influx of $\mathrm{Ca}^{2+}$ leads to the fusion of the opposing membranes. (d) Consequently, vesicles release the neurotransmitters into the synaptic cleft where they dock to receptors at the postsynaptic membrane. Adapted from Munson. ${ }^{[6]}$

But what is the underlying mechanisms by which the interaction of syb 2, syx 1A, and SNAP25 overcomes the energy barrier of fusion? Söllner et al. discovered a 1:1:1 stoichiometry of the three SNARE proteins, of which syb 2 is anchored inside the vesicular membrane via a transmembrane domain (TMD) and is therefore also called vesicular SNARE (v-SNARE). ${ }^{[4,8]}$ On the other site syx 1A and SNAP25 can be found at the presynaptic membrane (t-SNAREs), of which syx 1A also contains a TMD while SNAP25 is connected via a palmitoylated linker region to the target membrane. ${ }^{[8]}$ This linker region connects two 60-70 aa long coiled coil structures named SNARE-motifs that can also be found in syb 2 and syx 1A, the latter of which additionally contains a regulatory Habc domain connected to the $N$-terminus. ${ }^{[11]}$ These in total four SNARE motifs are highly conserved in the family of SNARE proteins and are, except for syx 1A, largely unstructured in solution (Figure 1.2 I) but form a very stable tetrameric complex with largely alphahelical content upon interaction (Figure 1.2 III). ${ }^{[5,12-15]}$ This complex contains 16 layers of mainly hydrophobic residues facing inwards, except for one hydrophilic 0-layer 
with three glutamine $(\mathrm{Q})$ and one arginine $(\mathrm{R})$ residue. ${ }^{[16-18]}$ Based on this 0-layer SNAREs are also divided into the four classes Qa,b,c and R-SNARE. The transformation from largely unstructured to a tight $4 \alpha$-helix bundle starts with the interaction of the $N$-termini of the SNARE domains and proceeds to the $C$-terminal end in a zippering kind of fashion (Figure 1.2 II). ${ }^{[19,20]}$ This process pulls the opposing membranes into close proximity and thus the energy released during complex formation subsequently leads to membrane fusion. ${ }^{[13,21,22]}$ During fusion the SNARE-complex changes its conformation from a high energy trans-configuration into a low energy cis-configuration where both TMDs are located inside the same lipid bilayer (Figure 1.2 II, III). ${ }^{[8]}$ The SNARE-complex is afterwards disassembled by the AAA+-ATPase NSF together with its SNAP receptor, vesicles and syb 2 retrieved from the target membrane, and liposomes reloaded with neurotransmitters to be ready for another fusion cycle. ${ }^{[7,8]}$

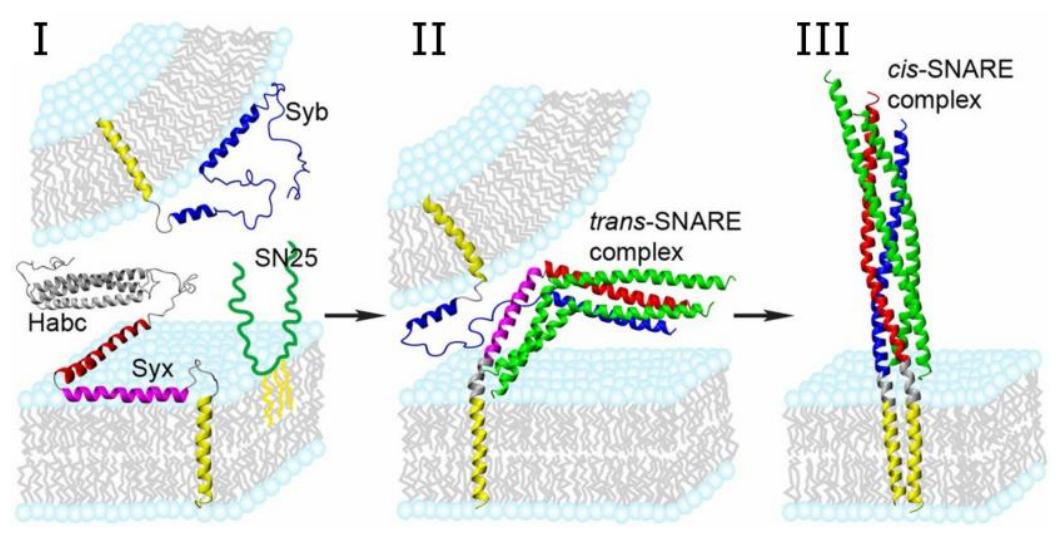

Figure 1.2 Models of the minimal fusion machinery at three stages of the fusion process derived from different microscopy and crystallography techniques (I) Prefusion structures of SNAP25 and syntaxin $1 \mathrm{~A}^{[11,23]}$ inside the target membrane and synaptobrevin 2 inside the vesicular membrane. ${ }^{[24]}$ (II) Models of the partly zippered transSNARE complex ${ }^{[20,23,24]}$ anchored in both membranes and (III) the cis-SNARE complex after fusion with both TMDs inside one bilayer. ${ }^{[20]}$ Modified according to Liang et al. ${ }^{[23]}$

Different mechanisms have been discussed of how exactly SNARE-zippering results in fusion pore formation and what the individual steps from vesicle docking until recycling are. ${ }^{[7,25,26]}$ In the first scenario SNAREs play a direct role in fusion pore formation, as their TMDs line the fusion pore and connect the two lumen under exclusion of lipids. ${ }^{[27]}$ However, this early idea proved to be unlikely due to the low number of SNAREs found to be necessary to drive membrane fusion. ${ }^{[28-30]}$ In the second scenario the close proximity of the two bilayers after SNARE-zippering leads to lipid splaying and the formation of a lipidic fusion stalk with lifetimes of $<1 \mathrm{~ms}$ (Figure 1.3). ${ }^{[31,32]}$ The stalk might enlarge into a hemifusion intermediate where only the lipids of the two outer leaflets of the opposing membranes are mixed. ${ }^{[33,34]}$ Whether this hemifusion state is a stable intermediate and when exactly an aqueous fusion pore is formed is, however, of constant debate. ${ }^{[33,35-37]}$ After a first aqueous connection the fusion 
pore can expand and the vesicle collapses into the target membrane. This classic fusion pathway is often referred to as full-collapse fusion (FC) and is accompanied by clathrin-mediated endocytosis and recycling of vesicles in endosomes or inside the cell plasma. ${ }^{[7]}$ However, an alternative fusion mode called kiss-and-run (KR) exocytosis involves a fusion pore that rapidly closes again before the vesicle collapses into the target membrane. ${ }^{[38]}$ In that way the shape of the liposome, also referred to as $\Omega$-shape, remains intact and proteins as well as lipid material are preserved. Subsequently, the vesicle is directly retrieved from the plasma membrane in a fast endocytosis mode. ${ }^{[26]}$ It is thought that KR is the dominant fusion mode at low frequency stimulation, however, the underlying mechanisms are still elusive and the relevance of KR is unclear. ${ }^{[38-41]}$

The large number of different proteins involved in these processes makes it difficult to unravel the molecular mechanisms that influence the fusion process in vivo. Consequently, it became obvious that in vitro studies are crucial to dissect the individual steps in the life cycle of the synaptic vesicle and to investigate the role of certain proteins, lipids, and other factors in a welldefined environment.

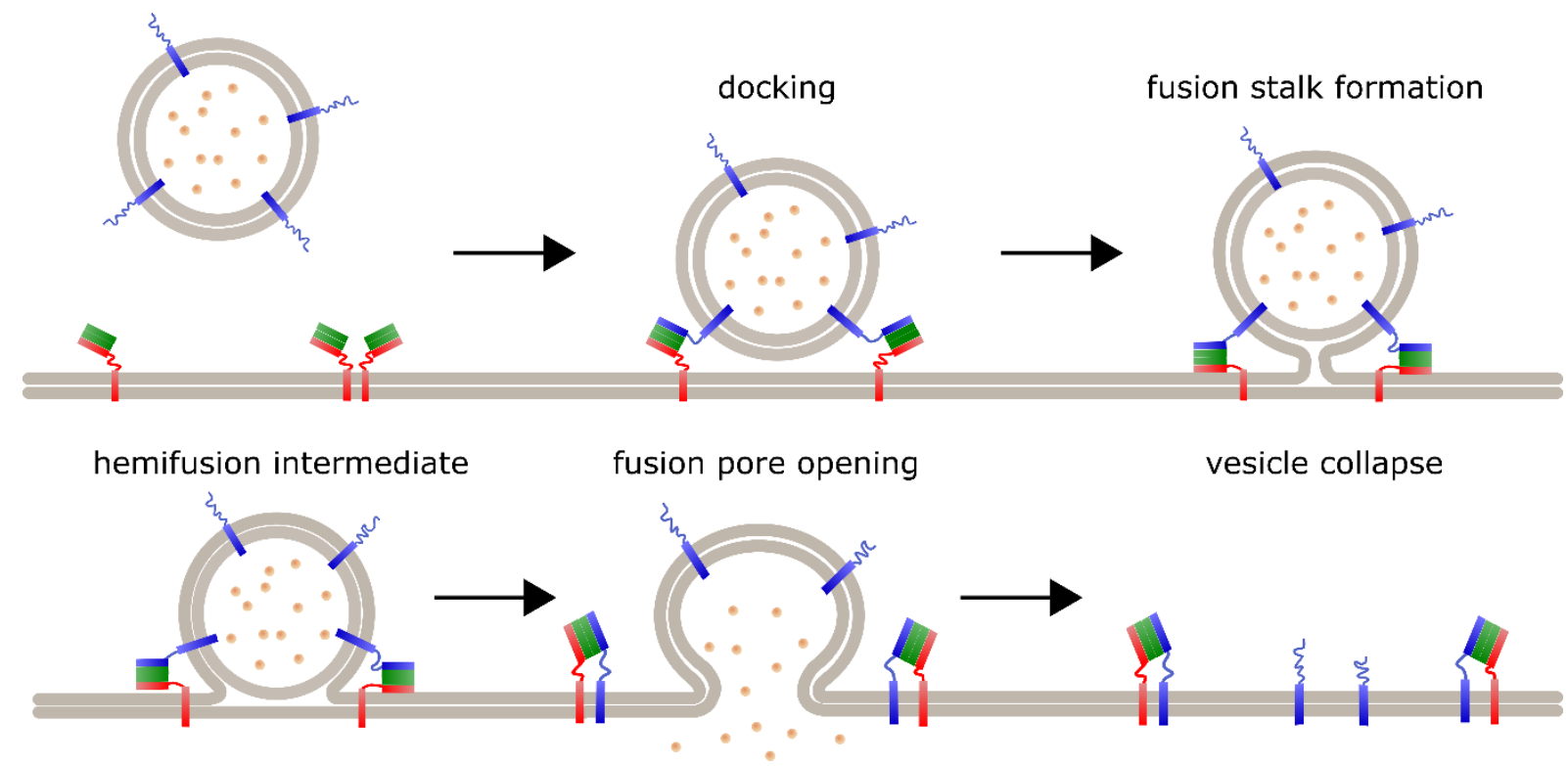

Figure 1.3 Model of a possible fusion pathway in SNARE-mediated membrane fusion. After vesicle docking and SNARE-zippering a fusion stalk is formed that evolves into a hemifusion intermediate. From this stable intermediate a fusion pore is formed, neurotransmitters are released, and the subsequent expansion of the pore leads to complete vesicle collapse.

\subsection{In vitro fusion assays}

The key feature of every in vitro fusion assay that involves the formation of a synthetic lipid bilayer in which proteins are incorporated is to ensure their functionality. This has led to the 
evolution of various reconstitution procedures, most of which are based on the detergent mediated destabilization/micellization of lipid structures to incorporate the protein of use followed by the removal of detergents. ${ }^{[42]}$ In addition, different variants of the minimal fusion machinery that are used to study SNARE-mediated membrane fusion have been developed to overcome the lack of regulatory proteins. For example, the $N$-terminal Habc domain of syx $1 \mathrm{~A}$ can bind back onto the SNARE-binding pocket and thus switches the protein between an active open and inactive closed state. ${ }^{[43-46]}$ As a consequence, syb 2 is incapable of binding to the t-SNARE complex which inhibits both docking as well as fusion. ${ }^{[47-49]}$ Furthermore, early studies showed that SNAP25 is capable of binding two molecules of syx 1A and that the subsequent binding of syb 2 is inhibited (Figure $1.4 \mathrm{~A}$ ). ${ }^{[13,50]}$ However, by using a mutated version of syx 1A lacking the Habc domain Pobbati et al. observed that fusion kinetics are drastically increased if a preformed acceptor complex is reconstituted into vesicles. ${ }^{[51]}$ In this so called $\Delta$ N49-complex syx $1 \mathrm{~A}$ and SNAP25 are mixed with a small fragment of syb 2 (aa 49-96) that binds to the $C$-terminal end of the SNARE-domain and prevents the binding of an additional syx 1A molecule (Figure $1.4 \mathrm{~B}$, left). As SNARE-zippering starts from the $N$-terminal end, full length syb 2 is able to displace the fragment from the SNARE-complex during the fusion process. These findings are similar to those obtained by Rothman and coworkers who detected an acceleration of fusion when the acceptor complex was pre-incubated with a small fragment of syb 2 called Vc peptide (aa 58-94). ${ }^{[52]}$ However, they explain these results with the preformation of the SNARE-complex from unstructured to largely $\alpha$-helical rather than the prevention of a dead end 2:1 complex. This is based on the observation that - in contrast to the mixing of syx 1A and SNAP25 in the plain of the lipid bilayer - the coexpression of syx 1A and SNAP25 solely leads to a 1:1 complex. Besides this coexpressed $1: 1$ and the $\Delta \mathrm{N} 49$-complex Tamm and coworkers recently developed two more acceptor SNARE complexes. ${ }^{[23,53]}$ They observed that isolation of syx 1A with dodecyl-phosphocholine leads to largely monomeric syx $1 \mathrm{~A}$ and also prevents the formation of an inactive 2:1 complex when used for the reconstitution of syx 1A and SNAP25 into liposomes (Figure 1.4 B, middle). In a different approach syx 1A and dodecylated SNAP25 were reconstituted separately into two liposome populations and the acceptor complex subsequently formed in the plane of the target membrane (Figure 1.4 B, right). These different minimal fusion machineries are employed to study the mechanisms of the SNARE-mediated fusion process in various fusion assays, many of which are based on the use of fluorophores which will thus be discussed in the following. 

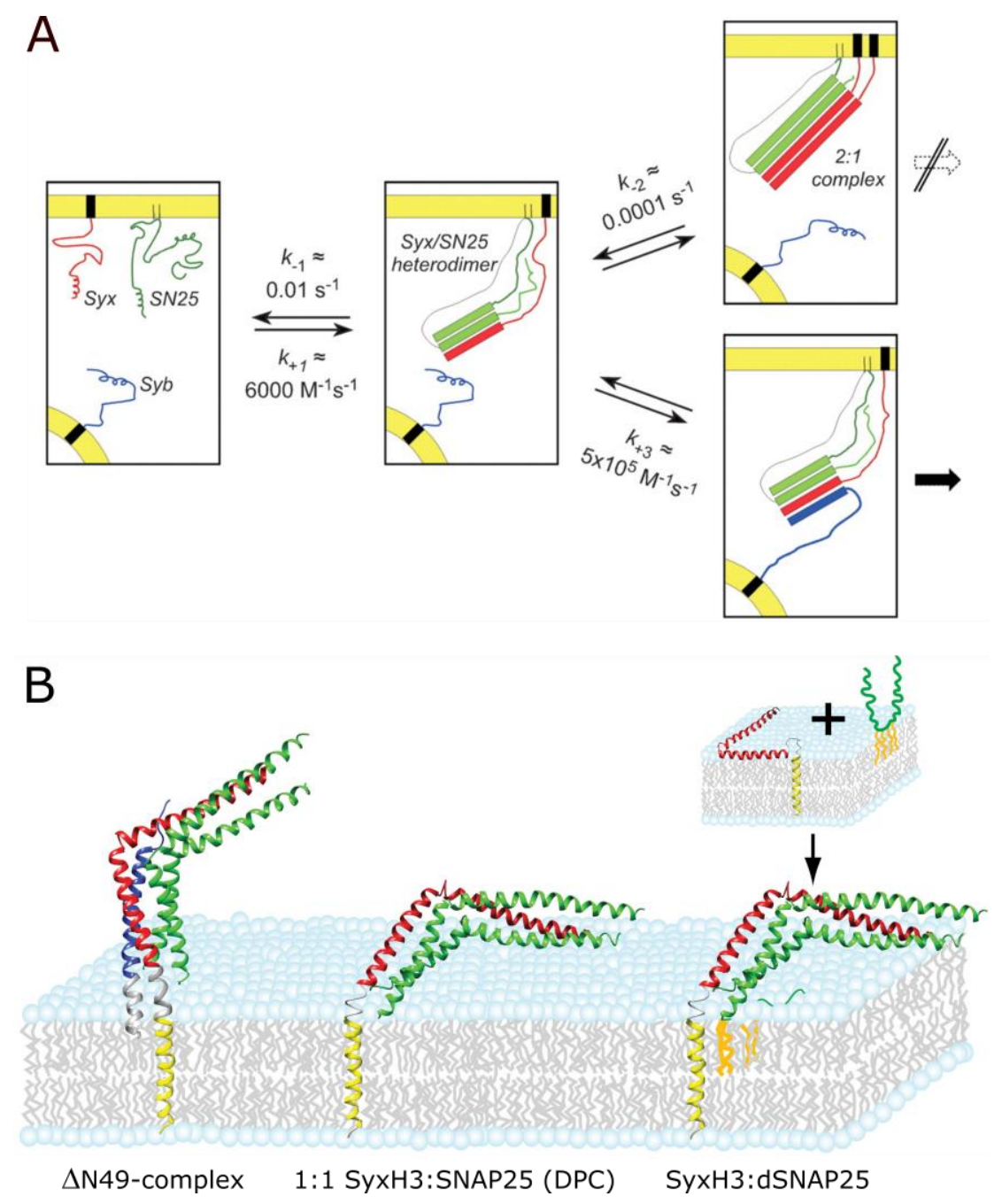

Figure 1.4 (A) Model of the assembly of SNARE proteins inside two opposing membranes (adapted from Pobbati et al.) including rate constants obtained from experiments with the soluble parts of the proteins. ${ }^{[50,51]}$ SNAP25 and syx 1A slowly form a 1:1 complex inside the plane of the target membrane to which syb 2 can rapidly bind. The binding of an additional syx 1A molecule to the heterodimer of syx 1A and SNAP25 leads to the formation of an unfusogenic 2:1 complex. (B) Schematic illustrations of three different acceptor complex types inside a lipid bilayer used to study membrane fusion. From left to right $\Delta$ N49-complex, Syx 1A:SNAP25 1:1 complex preassembled using dodecyl-phosphocholine, and 1:1 complex of syx 1A and dodecylated SNAP25 assembled in the plane of the membrane. ${ }^{[23,53]}$ Figure adapted from Kreutzberger et al. ${ }^{[53]}$

\subsubsection{Bulk fusion assays}

The first study to monitor SNARE-mediated membrane fusion in vitro was performed by Weber et al., is based on the observation of lipid mixing of two vesicle populations that contain either syb 2 or syx 1A and SNAP25 in a bulk solution, and led to the formulation of the SNARE-hypothesis. ${ }^{[10]}$ Based on the study of Struck et al. the fluorescence resonance energy transfer (FRET) pair $N$-(7-nitro-2,1,3-benzoxadiazole-4-yl)-phosphatidylethanolamine (NBD$\mathrm{PE}$, donor) and $\mathrm{N}$-(lissamine rhodamine $\mathrm{B}$ sulfonyl)-phosphatidylethanolamine (Rh-PE, acceptor) were incorporated into the membrane of one vesicle population (Figure $1.5 \mathrm{~A}) .{ }^{[54]}$ The fusion of vesicles leads to merging of the two opposing membranes which results in an 
increase of distance between the two lipid dyes and consequently an decrease in FRET-efficiency. If the FRET donor fluorescence is monitored as a function of time, successful lipid mixing is thus visible as an increase in fluorescence intensity. To distinguish in this assay between pure hemifusion, where only the outer leaflets of vesicles mix, and inner leaflet mixing, dithionite has to be added to the buffer solution. As dithionite efficiently quenches NBD fluorescence intensity and is incapable of penetrating through the lipid bilayer, a change in FRET efficiency can be directly correlated to inner leaflet mixing. ${ }^{[35,55,56]}$ However, a study performed in a DNA-mediated fusion setup revealed that inner leaflet mixing alone does not necessarily lead to content mixing. ${ }^{[57]}$ Thus, a more direct way of observing fusion pore formation is achieved by labeling the inner solution of one vesicle population with a water soluble fluorophore such as sulforhodamine B (SRB) in self-quenching concentrations (Figure $1.5 \mathrm{~B}) .{ }^{[58,59]}$ Here, the fusion of vesicles leads to the dilution of the quenched content marker and an increase in fluorescence intensity. As in both setups vesicle lysis and/or leakage is also leading to an increase in detected fluorescence, which cannot be separated from fusion, control measurements to check for vesicle stability are vital. ${ }^{[42]}$ Furthermore, osmotic differences between vesicle populations induced by the incorporation of ionic content markers can affect the fusion process. For example, a positive osmotic gradient (more ions inside the vesicle) reduced content mixing efficiency to $6 \%$ compared to iso-osmolar conditions in large unilamellar vesicle (LUV) fusion induced by poly ethylene glycol (PEG). ${ }^{[60]}$ Additionally, randomly flowing proteo-liposomes have only a certain probability to come in contact with each other in the bulk solution, hence extracted fusion kinetics are always dominated by the rate limiting step of the docking process. ${ }^{[29,30]}$ While careful considerations during sample preparation enabled the extraction of cooperative effects from observed kinetics, half-life times of the fusion process are usually in the range of several minutes ${ }^{[30,61]}$ up to hours. ${ }^{[62]}$ In addition, complex fusion intermediates and postfusion structures cannot be visualized in bulk fusion assays. 


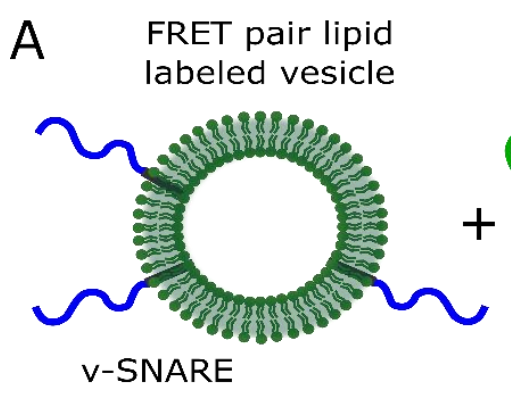

quenched donor

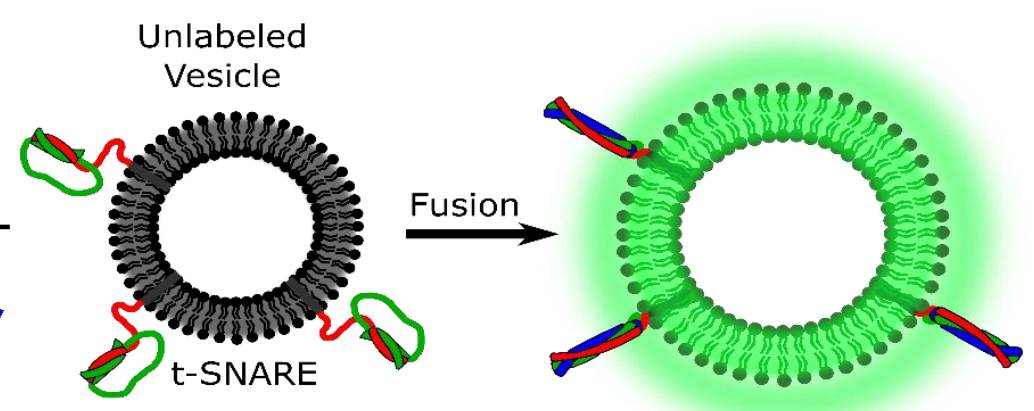

unquenched donor

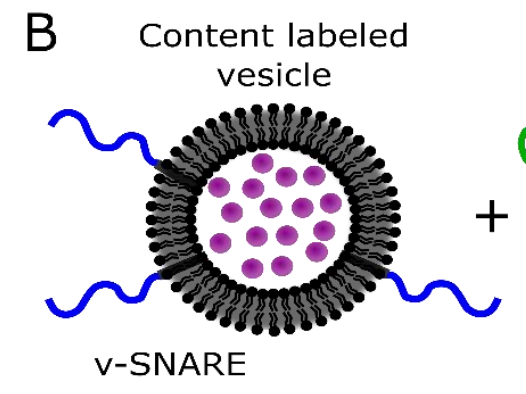

concentration

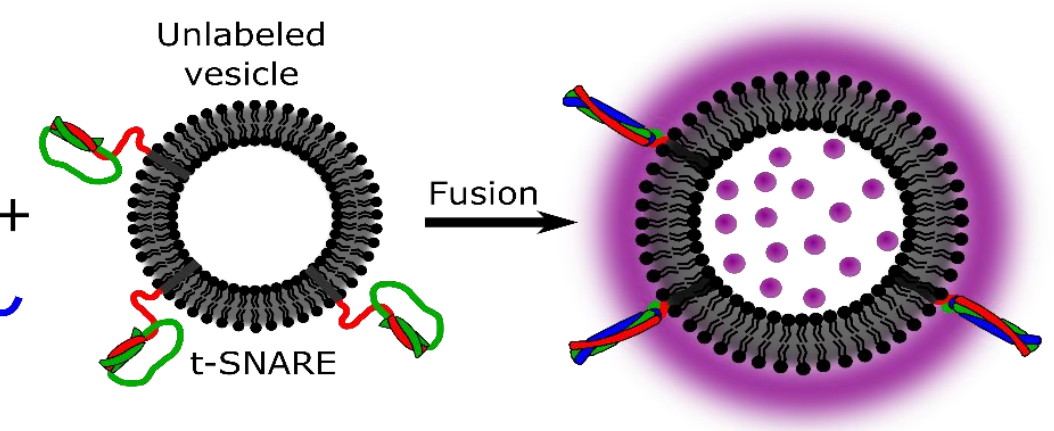

unquenched

quenched

Figure 1.5 Schematic illustrations of two exemplarily bulk fusion assays based on fluorescence spectroscopy. (A) Fusion is monitored by means of lipid mixing in a FRET-based assay where fusion leads to an increase in FRET donor fluorescence. (B) One vesicle population is filled with concentration quenched SRB and fusion leads to dilution of SRB and an increase in fluorescence.

\subsubsection{Single-vesicle fusion assays}

\section{Surface-adhered vesicles}

More detailed information about kinetics and fusion pathways can be obtained by monitoring the fusion process on a single-vesicle level. ${ }^{[42,63]}$ To this end, t-SNARE containing vesicles can be immobilized on a flat surface, v-SNARE doped vesicles added from the top, and the fusion of individual vesicles monitored over time (Figure 1.6 A). ${ }^{[64-69]}$ Here, fluorescence intensity time traces from individual docked vesicles are extracted by positioning a region of interest (ROI) on top of the docked vesicles. This allowed to directly differentiate between the three processes dead-end hemifusion, fusion pore formation, and vesicle bursting and it also enabled the detection of possible stable hemifusion intermediates. ${ }^{[64,67]}$ Additionally, complex fusion modes like the repetitive opening and closing of a fusion pore were visualized in a study of Gong et al. ${ }^{[68]}$ Despite these clear advantages over bulk fusion assays one major drawback of this approach is still the use of highly curved vesicles as the target membrane. Not only does 
this not resemble the geometric situation of the planar presynaptic membrane, several studies also showed that high vesicle curvature can affect their fusion behavior. ${ }^{[30,60,70,71]}$

\section{Supported lipid bilayers}

To produce a planar target membrane, vesicles can be spread on rather than adhered to a glass surface and in this way form a supported lipid bilayer (SLB). First developed to monitor SNARE-mediated fusion by Fix et al., SLBs were formed by direct adsorption of proteo-SUVs on pure glass surfaces and the fusion of v-SNARE doped SUVs imaged by means of total internal reflection fluorescence (TIRF) microscopy (Figure $1.6 \mathrm{~B}$ ). ${ }^{[48,72,73]}$ These early systems exhibited very low protein mobility and large immobile fractions inside the target membrane, probably due to direct contact of proteins with the solid support. ${ }^{[72]}$ Furthermore, Bowen et al. reported that fusion was enhanced by heating or laser induced radical formation of dyes and was additionally SNAP25 independent. Liu et al. also observed such a SNAP25 independent fusion, ${ }^{[73]}$ while in the study of Fix et al. efficient fusion was largely dependent on divalent cations without the respective regulatory proteins. ${ }^{\left[{ }^{48]}\right.}$ It is possible that immobile proteins caused these low fusion efficiencies and the observed SNARE-independency of the process. ${ }^{[42]}$ However, docking and fusion turned out to be SNARE dependent in a later study of Wang et $a l$. who used a more or less identical model system. ${ }^{[74]}$ Surprisingly, in this study vesicles most likely burst the content marker into the solution above the membrane rather than transferring it across the SLB. Wang et al. explain their findings with the lack of space underneath the target membrane that is not sufficient for the uptake of the vesicular content. To reduce these surface effects in SLBs, two main different approaches have been pursued in further studies. First, a $\sim 4 \mathrm{~nm}$ long PEG linker has been introduced between membrane and glass surface by Karatekin et al. to increase the available space underneath the membrane and to enhance protein mobility by uncoupling the SLB from the solid support (Figure $1.6 \mathrm{C}$ ). ${ }^{[75,76]}$ In this assay, the target membrane was labeled to control for defects formed during the spreading process and SNARE specific fusion was monitored by means of lipid mixing. The second method developed in the lab of Tamm and coworkers included the formation of a protein free monolayer using Langmuir-Blodgett transfer and the subsequent fusion of t-SNARE doped vesicles to this monolayer. ${ }^{[77]}$ Even though the target membrane is still in direct contact with the solid support, protein mobility is increased due to presumably quantitative orientation of the large cytosolic domains into the solution above the membrane. ${ }^{[78]}$ This assay proved to be useful to investigate the effect on fusion of a large variety of factors such as asymmetric phosphatidylethanolamine distribution inside the target membrane, vesicle size, and type of acceptor complex. ${ }^{[33,71,79-81]}$ 
While successful content transfer across the SLB was proven by modeling the efflux kinetic of content molecules, ${ }^{[82]}$ the unchanged very narrow space between membrane and solid support is likely to still influence the release kinetics as well as hinder the uptake of lipids of fusing vesicles. $^{[63]}$

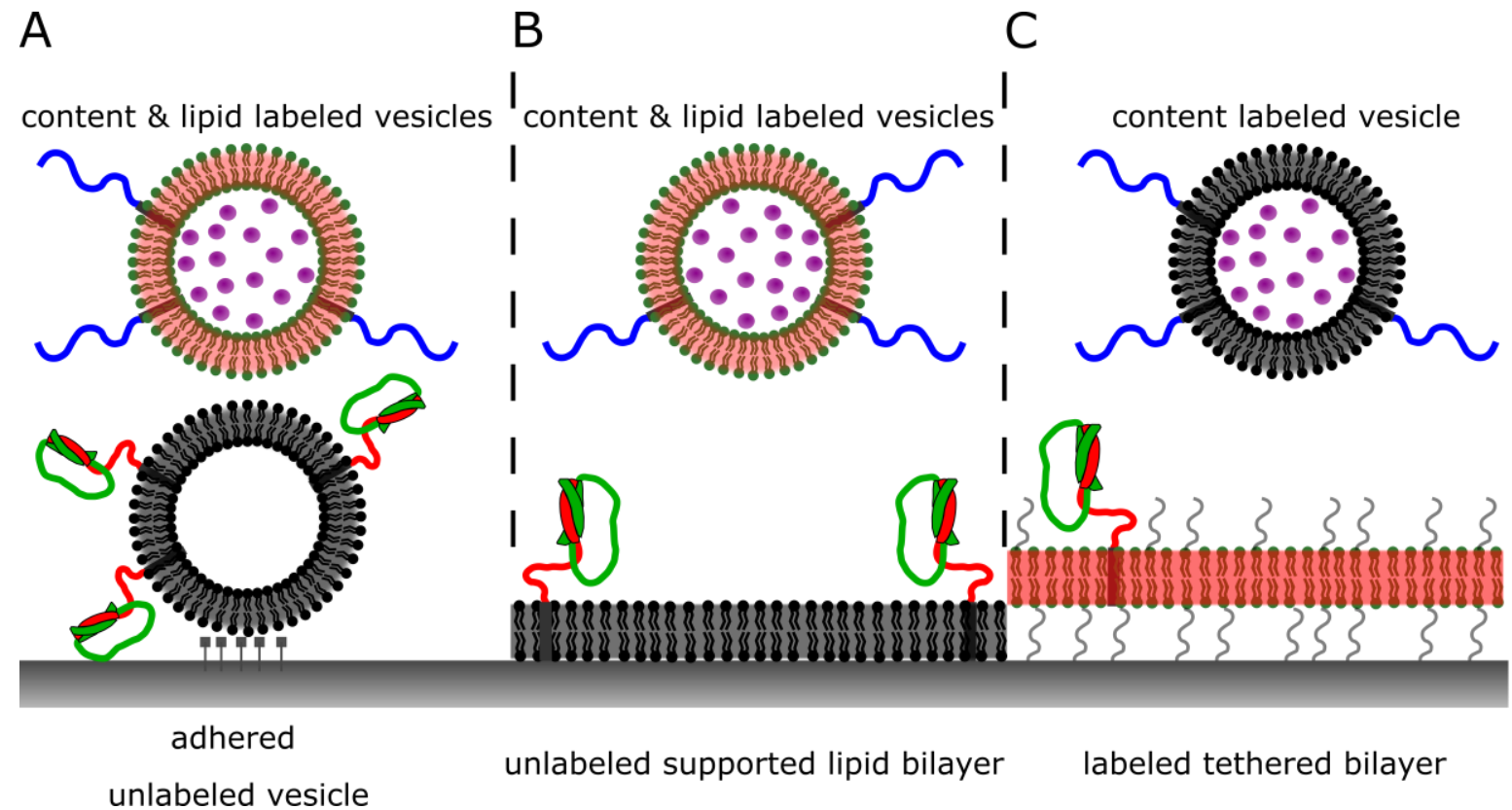

Figure 1.6 Schematic illustration of different single-vesicle content release fusion assays. (A) Fusion of syb 2 containing vesicles with t-SNARE doped liposomes that are adhered to a planar surface. (B) Setup in which vesicles fuse with a SLB that is in direct contact with the support. (C) A PEG-linker is introduced between support and bilayer to increase both protein mobility and the available space underneath the target membrane.

\section{Pore-spanning membranes}

A model system that combines the advantage of a planar target membrane which is easily accessible by means of fluorescence microscopy, and a $2^{\text {nd }}$ aqueous compartment for the vesicular content to be released in is called pore-spanning membranes (PSMs). ${ }^{[83]}$ Developed by Steinem and coworkers, PSMs are large, continuous bilayers that spread over a highly porous solid support and thus separate the bulk solution from the aqueous space underneath the freestanding part of the PSM (f-PSM). A first study performed by Höfer et al. proved their capability as a fusion assay and was further developed by Schwenen et al. to monitor fusion in a SNARE-dependent manner. ${ }^{[84,85]}$ Based on lipid mixing as a fusion indicator, meaningful information about possible fusion intermediates and postfusion structures were gathered with this setup. ${ }^{[85-87]}$ Furthermore, a study of Ramakrishnan et al. showed that PSMs are a useful tool to directly image unhindered content transfer across the target membrane. ${ }^{[62]}$ Thus, simultaneously imaging lipid mixing and content release in addition with high speed fluorescence microscopy is promising to gather meaningful information about the interplay 
between lipid mixing and fusion pore formation (Figure 1.7). This should enable to get deeper insight into the pathways of the SNARE-mediated fusion process and its kinetics.

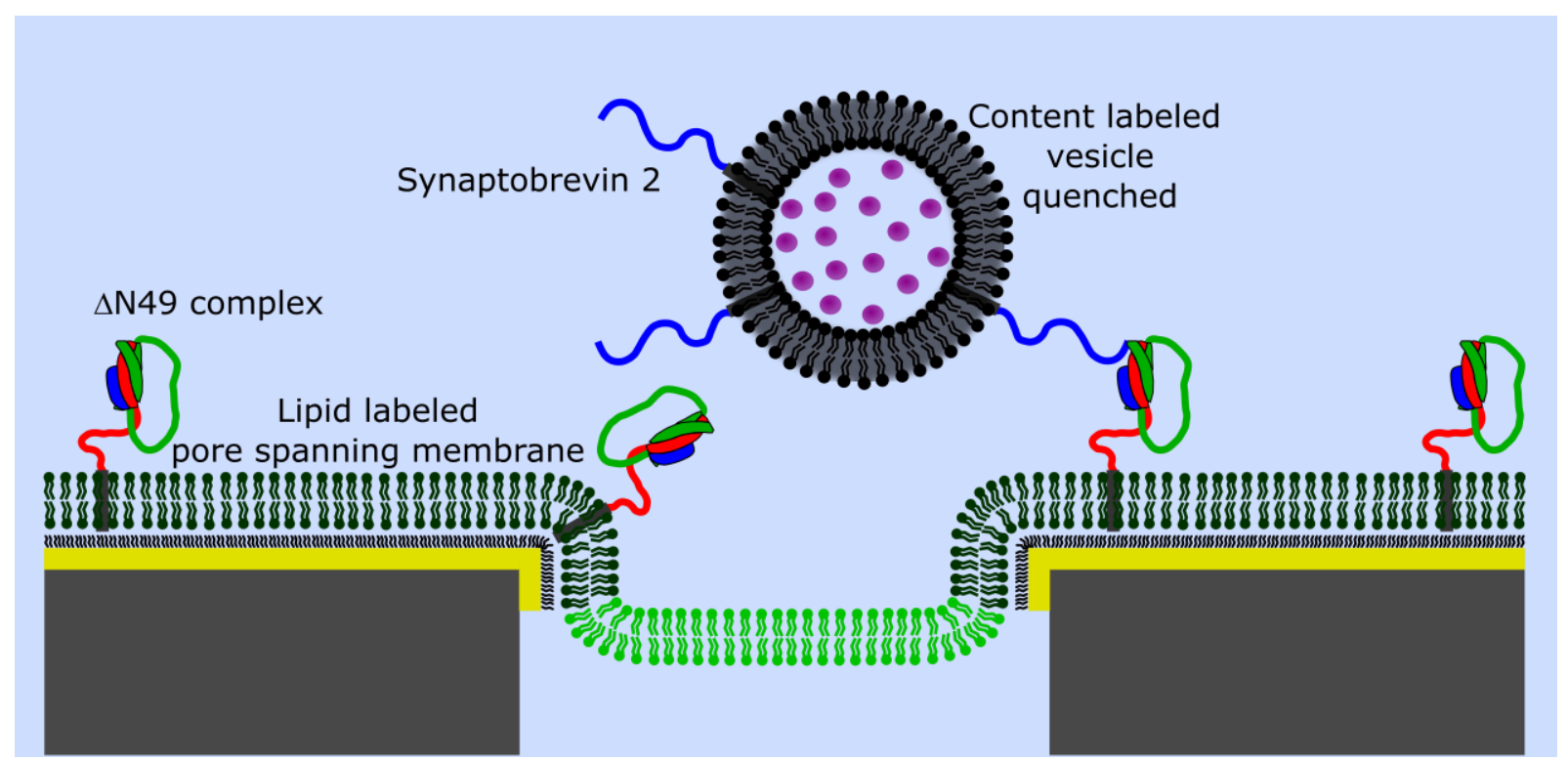

Figure 1.7 Schematic illustration of a SNARE-mediated fusion assay based on PSMs using a lipid labeled target membrane (green) and content labeled proteo-liposomes (magenta). Fluorescence on the solid support (s-PSM) is quenched due to the underlying gold functionalization. The PSM follows this surface at the edge of the pore rim and bends into the pore. 


\section{Scope}

During synaptic signal transmission neurotransmitter filled vesicles fuse with the presynaptic membrane to release their content into the synaptic cleft which in turn leads to the evolution of the action potential. At the core of this process stands the merging of the vesicular with the plasma membrane and the formation of an aqueous fusion pore through which small signaling molecules can diffuse. Early studies showed that this step is mediated by the interaction of the three SNARE (soluble $N$-ethylmaleimide-sensitive factor attachment receptor) proteins: synaptobrevin 2, syntaxin 1A and SNAP25 (synaptosomal associated protein of $25 \mathrm{kDa}$ ). However, the exact sequence of the individual steps from the first contact until the merging of the two membranes as well as the existence of different modes of fusion are still under constant debate.

Over the past two decades various in vitro model systems have been developed to unravel the influence of certain components on this complex process in a defined environment. Of these, as they combine advantages of different setups, pore-spanning membranes (PSMs) have been shown to be a useful tool to study SNARE-mediated single-vesicle fusion. PSMs are continuous planar lipid bilayers spread on a highly porous substrate and are composed of stabilizing solid supported (s-PSM) and freestanding parts (f-PSM) that cover large aqueous cavities. These $2^{\text {nd }}$ aqueous compartments make them unique compared to most other planar model membranes and render the process of content transfer across the target membrane potentially unhindered from an underlying solid support. In this work, PSMs containing the fusion active $\Delta \mathrm{N} 49$-complex will thus be used to gather meaningful information about the formation of the exocytotic fusion pore. To this end, the water soluble dye sulforhodamine B (SRB) will be encapsulated into syb 2 doped large unilamellar vesicles and the process of content release analyzed by means of high speed fluorescence microscopy. Simultaneous monitoring of lipid diffusion from the PSM into the vesicular membrane will be used to place key steps such as hemifusion, pore formation, and vesicle collapse in the temporal framework of the fusion process. Furthermore, different fusion pathways will be analyzed using large scale statistical analysis. In the last step, the impact of different factors such as apparent vesicle size and acceptor complex density on fusion pathways and kinetics will be investigated to get new insights in the underlying mechanisms of the SNARE-mediated fusion process. 


\section{Materials and Methods}

\subsection{Materials}

\subsubsection{Lipids and fluorescent probes}

\section{Lipids}

Phospholipids and cholesterol served as matrix lipids for the prepared model membranes used to study SNARE-mediated membrane fusion. V- and t-SNARE containing lipid bilayers were composed of DOPC/POPE/POPS/cholesterol in a molar ratio of 5/2/1/2 mimicking the composition in the plasma membrane of synapses and that of synaptic vesicles. ${ }^{[88-90]}$ Figure 3.1 shows the chemical structures of 1,2-dioleoyl-sn-glycero-3-phosphocholine (DOPC, $786.1 \mathrm{~g} / \mathrm{mol}, T_{\mathrm{m}}=-17^{\circ} \mathrm{C}$ ), 1-palmitoyl-2-oleoyl-sn-glycero-3-phosphoethanolamine (POPE, $718.0 \mathrm{~g} / \mathrm{mol}, \quad T_{\mathrm{m}}=25^{\circ} \mathrm{C}$ ), $\quad$ 1-palmitoyl-2-oleoyl-sn-glycero-3-phospho-L-serine $\quad$ (POPS, $\left.784.0 \mathrm{~g} / \mathrm{mol}, T_{\mathrm{m}}=14{ }^{\circ} \mathrm{C}\right)$ and cholesterol $(386.7 \mathrm{~g} / \mathrm{mol})$. Phospholipids were purchased from Avanti Polar Lipids (Alabaster, USA), cholesterol from Sigma-Aldrich (Taufkirchen, GER).

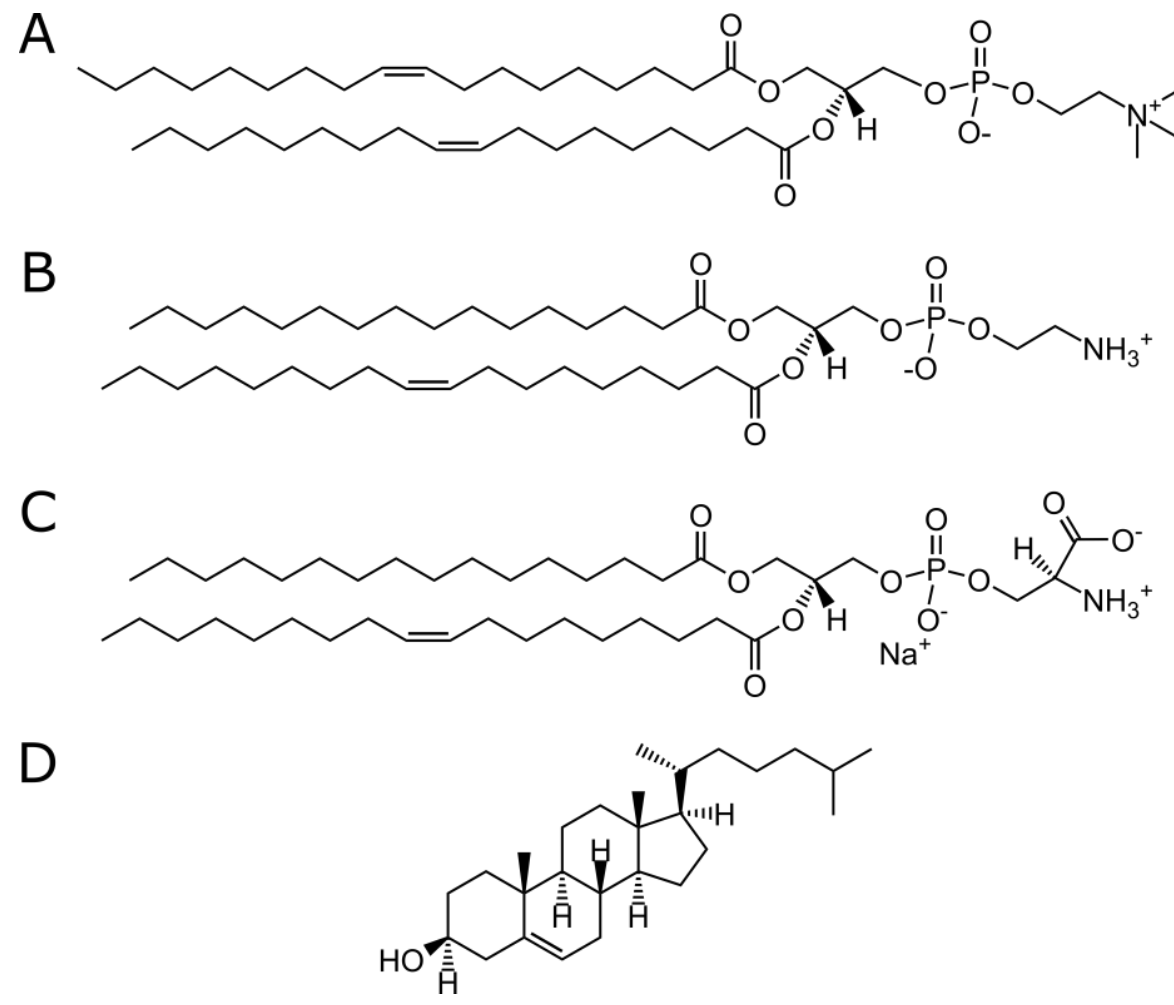

Figure 3.1 Structures of (A) 1,2-dioleoyl-sn-glycero-3-phosphocholine (DOPC, 786.1 g/mol), (B) 1-palmitoyl-2oleoyl-sn-glycero-3-phosphoethanolamine (POPE, $718.0 \mathrm{~g} / \mathrm{mol}$ ), (C) 1-palmitoyl-2-oleoyl-sn-glycero-3-phosphoL-serine (POPS, $784.0 \mathrm{~g} / \mathrm{mol}$ ) and (D) cholesterol $(386.7 \mathrm{~g} / \mathrm{mol})$ used to prepare lipid bilayers. 


\section{Fluorescent probes}

Different fluorescent molecules were used to study the mechanisms of SNARE-mediated fusion in single-vesicle experiments by means of fluorescence microscopy as well as to characterize important biophysical properties of the used model membrane systems. Depending on the application, different Atto-dyes coupled to the head group of 1,2-dipalmitoyl-sn-glycero-3phosphoethanolamine (DPPE) were used to label the lipid bilayer. Due to its high photostability, 1 mol\% of Atto655 DPPE was used to label PSMs for most single-vesicle fusion experiments, while Atto390/488 DPPE were used for fluorescence recovery after photobleaching (FRAP) experiments due to their lower photostability. Sulforhodamine B acid chloride (SRB) was used due to its high water solubility and photostability to fluorescently label the interior solution of large unilamellar vesicles (LUVs) for the observation of content release during single-vesicle fusion experiments. Chemical structures of lipid coupled dyes purchased from Atto-Tec (Siegen, GER) as well as SRB purchased from Sigma-Aldrich (Taufkirchen, GER) are shown in Figure 3.2 and Figure 3.3 respectively.

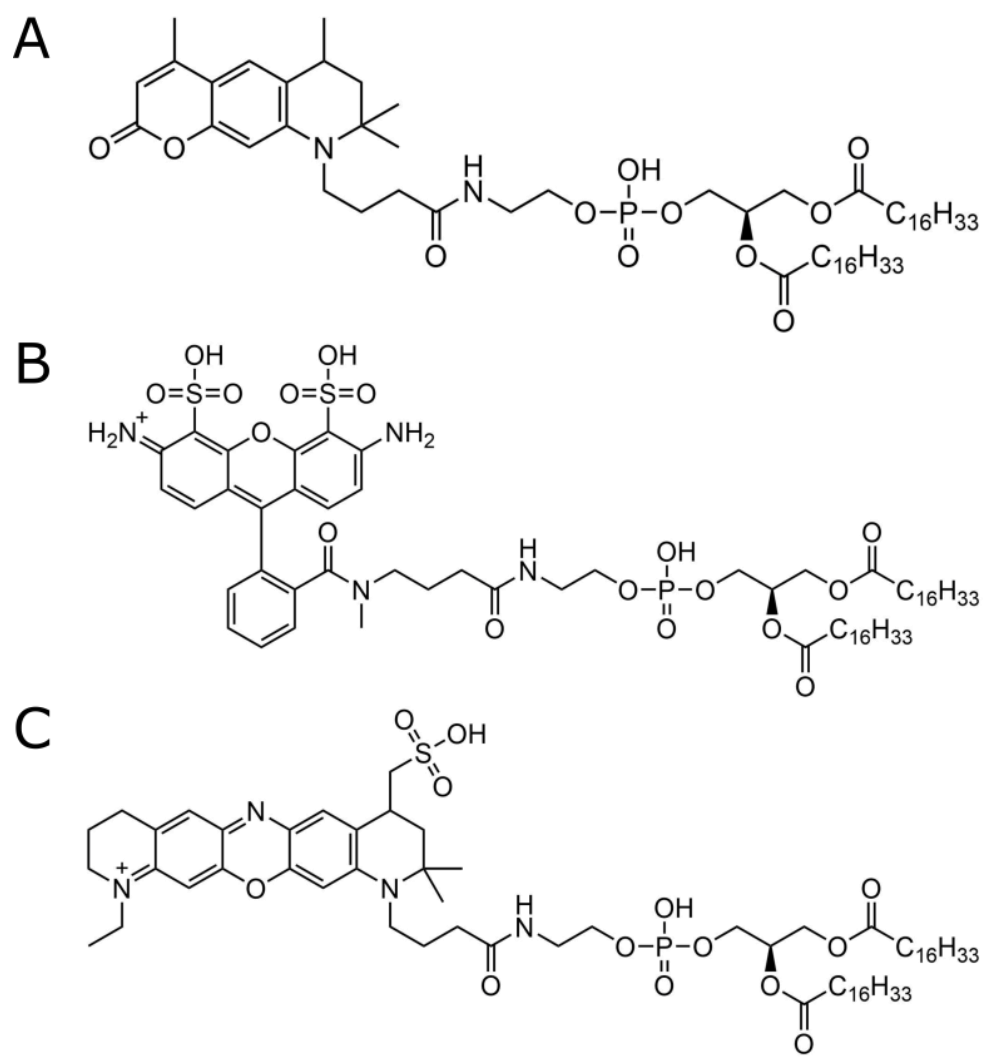

Figure 3.2 Structures of lipid-coupled fluorophores (A) Atto390-1,2-dipalmitoyl-sn-glycero-3phosphoethanolamine (Atto390-DPPE, $1017 \mathrm{~g} / \mathrm{mol}, \quad \lambda_{\mathrm{abs}}=390 \mathrm{~nm}, \quad \lambda_{\mathrm{em}}=476 \mathrm{~nm}$ ) (B), Atto488-DPPE $\left(1263 \mathrm{~g} / \mathrm{mol}, \lambda_{\text {abs }}=500 \mathrm{~nm}, \lambda_{\mathrm{em}}=520 \mathrm{~nm}\right)$ and $(\mathrm{C})$ Atto655-DPPE $\left(1315 \mathrm{~g} / \mathrm{mol}, \lambda_{\text {abs }}=663 \mathrm{~nm}, \lambda_{\mathrm{em}}=680 \mathrm{~nm}\right)$ to label lipid bilayers. 


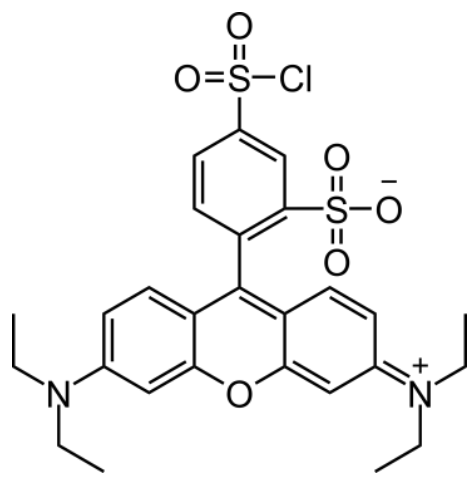

Figure 3.3 Structure of sulforhodamine B acid chloride $\left(\mathrm{SRB}, 577.11 \mathrm{~g} / \mathrm{mol}, \lambda_{\mathrm{abs}}=560 \mathrm{~nm}, \lambda_{\mathrm{em}}=590 \mathrm{~nm}\right)$.

\subsubsection{SNARE proteins}

To study the process of membrane fusion, the key step in neuronal signal transmission, full-length synaptobrevin 2 (syb 2) was incorporated into vesicles and their fusion with pore-spanning membranes (PSMs), containing a fusion active t-SNARE complex, monitored on a single-vesicle level. The t-SNARE complex $\Delta \mathrm{N} 49$ developed by Pobbati et al. was assembled from syntaxin 1A (syx 1A, aa 183-288), lacking the regulatory Habc domain, a version of full-length SNAP25 (SNAP25a, aa 1-206) with all cysteine residues (aa 84, 85, 90, 92) replaced by serine, and a small fragment of syb 2 (aa 49-96). ${ }^{[51]}$ As seen in Figure 3.4 , the small fragment binds to the SNARE binding pocket of full-length syb 2. ${ }^{[61]}$ The syb fragment prevents the binding of an additional syx 1A molecule to the free binding pocket of syb 2 during protein reconstitution as this so called 2:1 complex of syx 1A and SNAP25a would be nonfusogenic. ${ }^{[51]}$ The small fragment leaves the $N$-terminal SNARE-binding domain of the $\Delta$ N49complex free, allowing for full-length syb 2 to bind and displace the small fragment during the fusion process. A longer syb 2 fragment (aa 1-96) can, however, displace the small fragment and then occupies the whole binding pocket and as a results it cannot be replaced by full-length syb 2. ${ }^{[51]}$ This blocked fusion inactive t-SNARE complex is used for control measurements during this work.

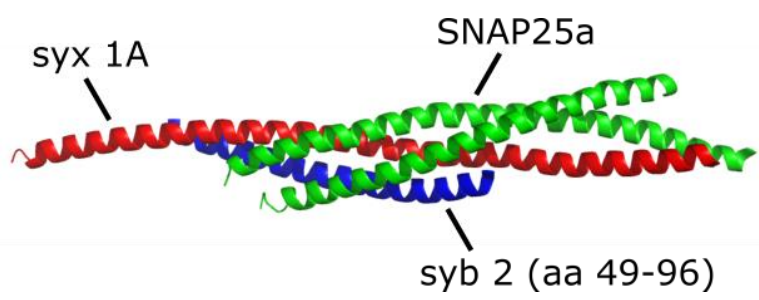

Figure 3.4 Ribbon plot of the t-SNARE $\triangle$ N49 acceptor complex with the synaprobrevin 2 fragment (blue, aa 4996), syntaxin 1A (red, aa 183-288) and SNAP25a (green, aa 1-206, all cysteine residues replaced by serine). ${ }^{\text {[61] }}$ 


\section{Synaptobrevin 2}

The v-SNARE syb $2\left(12.691 \mathrm{kDa}, \mathrm{pI}=7.84, \varepsilon=13980 \mathrm{M}^{-1} \mathrm{~cm}^{-1}\right.$, Figure 3.5$)$ is anchored inside the vesicular membrane via a transmembrane domain (aa 95-114) with aa 1-91 reaching into the cytosol and containing the SNARE-motif (aa 31-91). A truncated version of syb 2 (aa 49-96, $5.627 \mathrm{kDa}, \mathrm{pI}=9.16, \varepsilon=12490 \mathrm{M}^{-1} \mathrm{~cm}^{-1}$ ) was used to assemble the t-SNARE acceptor complex $\Delta \mathrm{N} 49$ while the complex was blocked for control experiments with the soluble part of syb 2 (aa 1-96, $\left.10.517 \mathrm{kDa}, \mathrm{pI}=8.04, \varepsilon=12660 \mathrm{M}^{-1} \mathrm{~cm}^{-1}\right)$. A mutated version of the small syb 2 fragment (aa 49-96 S79C, $5643 \mathrm{kDa}, \mathrm{pI}=8.90, \varepsilon=12490 \mathrm{M}^{-1} \mathrm{~cm}^{-1}$ ) was used to fluorescently label the fragment and subsequently the acceptor complex with Atto488 maleimide.

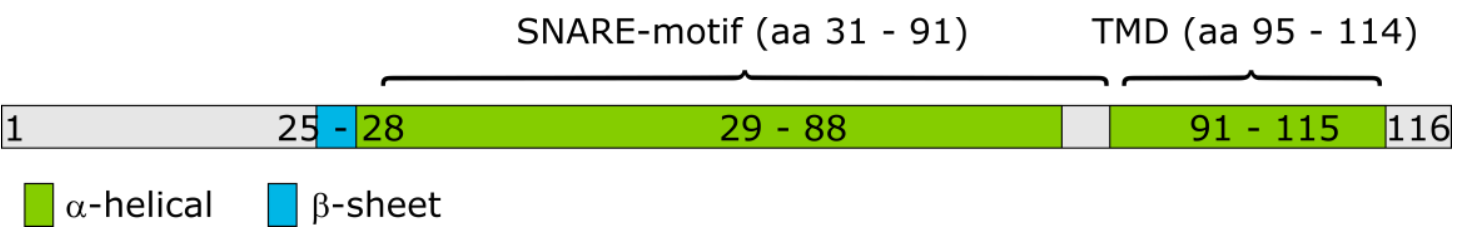

Figure 3.5 Schematic illustration of the secondary structure of the v-SNARE synaptobrevin 2 (aa 1-116) with unstructured parts shown in grey, $\alpha$-helical parts in green and $\beta$-sheets in blue. ${ }^{[15]}$

\section{Syntaxin 1A}

The t-SNARE syx 1A (aa 183-288, $12.035 \mathrm{kDa}, \mathrm{pI}=5.90, \varepsilon=4470 \mathrm{M}^{-1} \mathrm{~cm}^{-1}$, Figure 3.6) is anchored inside the plasma membrane via a transmembrane domain (aa 266-288) with aa 183-254 reaching into the cytosol and containing the SNARE-motif (aa 192-254). The truncated version used in this work lacks the regulatory Habc domain.

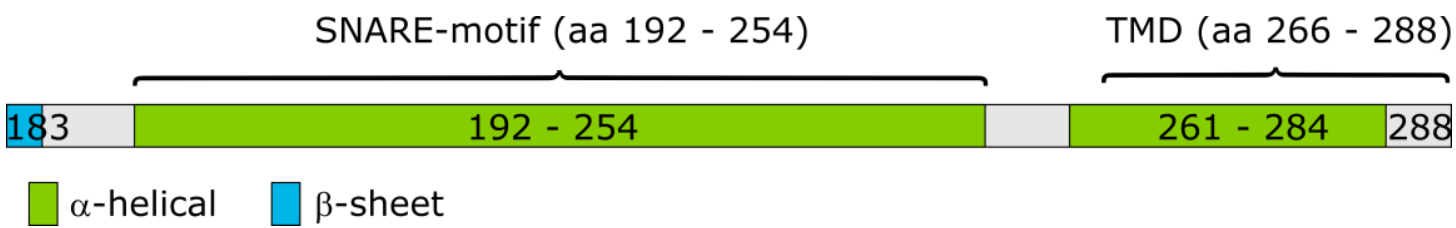

Figure 3.6 Schematic illustration of the secondary structure of the t-SNARE syntaxin 1A (aa 183-288) with unstructured parts shown in grey, $\alpha$-helical parts in green and $\beta$-sheets in blue. ${ }^{[15]}$

\section{SNAP25a}

In vivo, $\mathrm{t}-\mathrm{SNARE}$ SNAP25a (aa 1-206, $23.207 \mathrm{kDa}, \mathrm{pI}=4.74, \varepsilon=7240 \mathrm{M}^{-1} \mathrm{~cm}^{-1}$, Figure 3.7) is anchored peripherically at the plasma membrane via a palmitoylated linker connecting its two SNARE-motifs. In this work a mutated version was used with all cysteine residues replaced by serine (aa $84,85,90,92)$ since the protein could not be translationally modified with the used expression system and free cysteine residues would eventually lead to inter and 
intramolecular cysteine bridges. In contrast to syb 2 and syx 1A, SNAP25 contains two SNARE-motifs (aa 19-81 and aa 140-202).

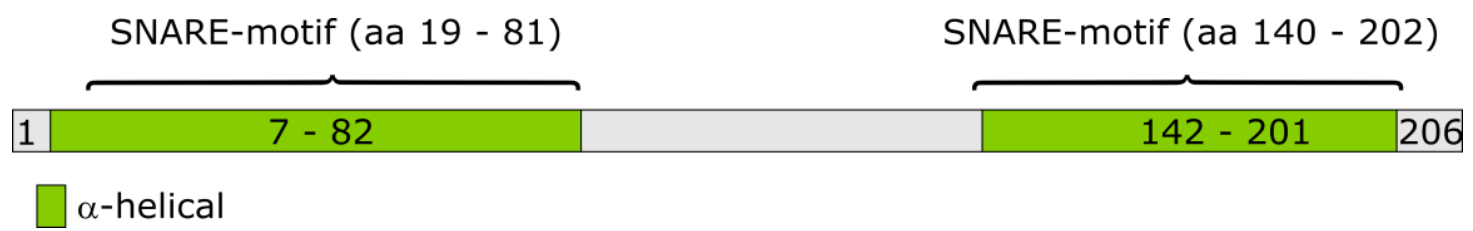

Figure 3.7 Schematic illustration of the secondary structure of the t-SNARE SNAP25a (aa 1-206) with unstructured parts shown in grey and $\alpha$-helical parts in green. ${ }^{[15]}$

\subsection{Preparative methods}

\subsubsection{SDS-polyacrylamide gel electrophoresis}

Sodium dodecyl sulfate polyacrylamide gel electrophoresis (SDS-PAGE) was used as a qualitative measure of successful protein purification. Proteins denatured with the detergent SDS are added on a polyacrylamide gel and are separated by their mass. The process is driven by applying an electric field to the gel that results in the movement of charged particles towards the anode. Since one protein molecule is surrounded by many charged SDS-molecules its own charge can be neglected and the velocity with which the protein travels inside the electric field is solely dependent on its mass.

Experimental procedure

Schägger gels, containing a stacking and a separating part, were used in this work to analyze the mass and purity of proteins. ${ }^{[91,92]}$ First, the separating gel was mixed according to Table 3-1 with buffers listed in Table 3-2 and filled into a casting chamber. The separating gel was then gently overlayed with the stacking gel (Table 3-1), both gels polymerized completely, the casting chamber disassembled, and the gel stored at $4{ }^{\circ} \mathrm{C}$ for up to 2 weeks. 
Table 3-1 Composition of stacking and separating gel for SDS-PAGE analysis.

\begin{tabular}{l|l|l} 
& Stacking gel $/ \mu \mathrm{L}$ & Separating gel $/ \mu \mathrm{L}$ \\
\hline Gel buffer & 281.25 & 1250 \\
$\mathrm{H}_{2} \mathrm{O}$ & 693.75 & 427.5 \\
Glycerin $(50 \%(v / v))$ & --- & 795 \\
Acrylamide solution & 150 & 1250 \\
TEMED $(99.9 \%(v / v))$ & 1.5 & 2.25 \\
APS $(10 \%(w / v))$ & 7.5 & 18.75
\end{tabular}

Table 3-2 Composition of buffers used for SDS-PAGE analysis.

\begin{tabular}{|c|c|c|}
\hline & Chemical & Concentration \\
\hline \multirow[t]{3}{*}{ Cathode buffer (10x) } & TRIS & $1 \mathrm{M}$ \\
\hline & Tricin & $1 \mathrm{M}$ \\
\hline & SDS & $1 \%(w / v)$ \\
\hline Anode buffer (10x) & TRIS/HCl & $2 \mathrm{M}, \mathrm{pH}=8.90$ \\
\hline \multirow[t]{2}{*}{ Gel buffer } & TRIS/HCl & $3 \mathrm{M}, \mathrm{pH}=8.45$ \\
\hline & SDS & $0.3 \%(w / v)$ \\
\hline \multirow[t]{5}{*}{ Sample buffer $(2 x)$} & TRIS/HCl & $175 \mathrm{mM}, \mathrm{pH}=6.80$ \\
\hline & SDS & $5 \%(w / v)$ \\
\hline & Glycerin & $15 \%(w / v)$ \\
\hline & DTT & $0.3 \mathrm{M}$ \\
\hline & Bromphenole blue & $0.02 \%(w / v)$ \\
\hline \multirow[t]{2}{*}{ Coomassie solution } & Coomassie G-250 & $80 \mathrm{mg} \mathrm{L}^{-1}$ \\
\hline & $\mathrm{HCl}$, conc. & $3 \mathrm{~mL}$ \\
\hline \multirow[t]{2}{*}{ Acrylamide solution } & Acrylamide & $30 \%(w / v)$ \\
\hline & Bisacrylamide & $0.8 \%(w / v)$ \\
\hline
\end{tabular}


Depending on the approximate protein concentration, samples were mixed according to Table 3-3, incubated for $10 \mathrm{~min}$ at $95{ }^{\circ} \mathrm{C}$ and $300 \mathrm{rpm}$ (Thermomixer compact, Eppendorf), and loaded into the gel. $70 \mathrm{~V}$ were applied for $10 \mathrm{~min}$ that allowed proteins to form a sharp band inside the stacking gel, then the voltage was increased to $200 \mathrm{~V}$ for $\sim 50 \mathrm{~min}$, and proteins separated by their mass. The gel was washed with $\mathrm{H}_{2} \mathrm{O}$, heated shortly to $\sim 100{ }^{\circ} \mathrm{C}$, and protein bands were visualized with Coomassie brilliant blue solution. Excess of staining solution was extracted from the gel by incubation in $\mathrm{H}_{2} \mathrm{O}$ overnight.

Table 3-3 Composition of samples for SDS-PAGE.

\begin{tabular}{l|l} 
& Volume $/ \mu \mathrm{L}$ \\
\hline Protein & $\mathrm{x}$ \\
Sample buffer (2x) & 6 \\
$\mathrm{H}_{2} \mathrm{O}$ & $5-\mathrm{x}$
\end{tabular}

\subsubsection{Chromatography}

\section{Ion exchange chromatography}

Ion exchange chromatography (IEC) was used as the final step of protein purification and is based on the different binding affinities of proteins inside a fluid phase towards a charged stationary phase depending on their net surface charge. To be able to bind to the stationary phase the $\mathrm{pH}$ of the used buffer solution has to differ from the isoelectric point ( $\mathrm{pI}$ ) of the protein. If the protein is e.g. negatively charged, a positively charged stationary phase is used (anion exchanger). Impurities with different pI then elute from the column at different ionic strengths of the fluid phase. Thus, slowly increasing the salt concentration of the fluid phase leads to the separation of the pure protein from impurities.

Experimental procedure

In this work, an ÄKTA protein purification system (GE Healthcare, Little Chalfont, UK) was used to perform IEC. Proteins were diluted in water or a low salt buffer to be able to bind effectively to the stationary phase which was either an anion exchange column MonoQ (SNAP25a, syx 1A, $\Delta$ N49-complex) or a cation exchange column MonoS (full-length syb 2, syb 2 49-96, syb 2 49-96 S79C). The column was equilibrated in ÄKTA buffer A and the protein bound under constant flow to the stationary phase. Increasing the concentration to high 
salt ÄKTA buffer B in a pattern specific for each individual protein leads to the elution of the purified protein. Elution from the column is detected by continuously measuring the absorption at $280 \mathrm{~nm}$ and purity controlled by means of SDS-PAGE analysis.

\section{Size exclusion chromatography}

Size exclusion chromatography was used for the purification of proteins and during the reconstitution process of proteins into liposomes. Molecules inside the fluid phase are continuously flushed over a stationary phase consisting of a highly porous mesh. Small molecules enter these pores more easily than larger particles and elute later from the stationary phase.

\subsubsection{SNARE protein isolation and purification}

DNA sequences of SNARE proteins incorporated into pET28a(+) vectors originated from rattus norvegicus and were provided by the group of Reinhard Jahn (MPI for Biophysical Chemistry, Göttingen, Germany). Heterogeneous protein expression was performed in E. coli (BL21 (DE3)) and His6-tag containing proteins purified via Nickel affinity chromatography and IEC. The detailed purification protocol including all SDS-PAGE analysis is exemplarily described for Sb49-96 S79C. Due to the large similarity of purification protocols only the final IEC chromatogram, the respective SDS-PAGE, and eventual modifications of the protocol are reported for the remaining proteins. Compositions of buffers and media for the isolation and purification of SNARE proteins are listed in Table 3-4 and Table 3-5. 
Table 3-4 Composition of buffers for the isolation and purification of SNARE proteins.

\begin{tabular}{|c|c|c|}
\hline & Chemical & Concentration \\
\hline \multirow[t]{3}{*}{ Resuspension buffer } & TRIS/HCl & $20 \mathrm{mM}, \mathrm{pH}=7.4$ \\
\hline & $\mathrm{NaCl}$ & $500 \mathrm{mM}$ \\
\hline & Imidazole & $20 \mathrm{mM}$ \\
\hline \multirow[t]{4}{*}{ Extraction buffer } & TRIS/HCl & $20 \mathrm{mM}, \mathrm{pH}=7.4$ \\
\hline & $\mathrm{NaCl}$ & $500 \mathrm{mM}$ \\
\hline & Imidazole & $20 \mathrm{mM}$ \\
\hline & Sodium cholate & $10 \%(w / v)$ \\
\hline \multirow[t]{4}{*}{ Elution buffer } & TRIS/HCl & $20 \mathrm{mM}, \mathrm{pH}=7.4$ \\
\hline & $\mathrm{NaCl}$ & $500 \mathrm{mM}$ \\
\hline & Imidazole & $400 \mathrm{mM}$ \\
\hline & DTT & $1 \mathrm{mM}$ \\
\hline \multirow[t]{4}{*}{ Dialysis buffer } & TRIS/HCl & $20 \mathrm{mM}, \mathrm{pH}=7.4$ \\
\hline & $\mathrm{NaCl}$ & $150 \mathrm{mM}$ \\
\hline & EDTA & $1 \mathrm{mM}$ \\
\hline & DTT & $1 \mathrm{mM}$ \\
\hline \multirow[t]{3}{*}{ ÄKTA buffer A } & HEPES/NaOH & $20 \mathrm{mM}, \mathrm{pH}=7.4$ \\
\hline & EDTA & $1 \mathrm{mM}$ \\
\hline & DTT & $1 \mathrm{mM}$ \\
\hline \multirow[t]{4}{*}{ ÄKTA buffer B } & HEPES/NaOH & $20 \mathrm{mM}, \mathrm{pH}=7.4$ \\
\hline & $\mathrm{NaCl}$ & $1 \mathrm{M}$ \\
\hline & EDTA & $1 \mathrm{mM}$ \\
\hline & DTT & $1 \mathrm{mM}$ \\
\hline
\end{tabular}


Table 3-5 Composition of expression media for the pre- and main culture.

\begin{tabular}{l|l|l} 
& Chemical & Concentration \\
\hline LB-medium & Tryptone & $10 \mathrm{~g} / \mathrm{L}$ \\
Yeast extract & $5 \mathrm{~g} / \mathrm{L}$ \\
$\mathrm{NaCl}$ & $5 \mathrm{~g} / \mathrm{L}$ \\
TB-medium & Tryptone & $13.5 \mathrm{~g} / \mathrm{L}$ \\
Yeast extract & $26.5 \mathrm{~g} / \mathrm{L}$ \\
Tlycerin & $4.5 \mathrm{~g} / \mathrm{L}$ \\
& $\mathrm{K}_{2} \mathrm{HPO}_{4}$ & $125 \mathrm{~g} / \mathrm{L}$ \\
$\mathrm{KH}_{2} \mathrm{PO}_{4}$ & $23 \mathrm{~g} / \mathrm{L}$
\end{tabular}

\section{Sb49-96 S79C}

Expression

Protein expression was carried out under sterile conditions and in all buffers 1 mM DTT was exchanged by $0.1 \mathrm{mM}$ TCEP. $30 \mu \mathrm{g} / \mathrm{mL}$ kanamycin were added to $2 \times 75 \mathrm{~mL}$ of autoclaved LB-medium and pre-cultures cultivated overnight $\left(37^{\circ} \mathrm{C}, 175 \mathrm{rpm}\right)$ using glycerol stocks of previous expressions. On the next day, 10 x $225 \mathrm{~mL}$ TB-medium were each mixed with $25 \mathrm{~mL}$ of TB-salt and kanamycin $(30 \mu \mathrm{g} / \mathrm{mL})$ and inoculated with each $5 \mathrm{~mL}$ of pre-culture. The main culture was incubated until an optical thickness at $600 \mathrm{~nm}\left(O D_{600}\right)$ of $0.9-1$ was reached $\left(37^{\circ} \mathrm{C}\right.$, $185 \mathrm{rpm})$. Lac-operon induced protein expression was started by adding Isopropyl- $\beta$-Dthiogalactopyranoside (IPTG, final concentration $0.25 \mathrm{mM}$ ) and performed for $3 \mathrm{~h}$ at $37{ }^{\circ} \mathrm{C}$ and $185 \mathrm{rpm}$. The temperature was reduced to $15^{\circ} \mathrm{C}$ and main cultures centrifuged stepwise (20 min, $4{ }^{\circ} \mathrm{C}, 4500 \mathrm{rpm}$ ) to obtain cell pellets. Cell pellets were separated from the supernatant, resuspended in resuspension buffer $\left(15 \mathrm{~mL}\right.$ for each pellet), and stored overnight at $-20{ }^{\circ} \mathrm{C}$.

\section{Extraction}

Lysozyme (4 mg/L main culture), $\mathrm{MgCl}_{2}$ (1 mM), DNase 1 (2 mg), cOmplete (1 tablet, EDTA free) were added to the unfrozen cell pellets and the mixture was stirred to start cell lysis (30 min, RT). The suspension was homogenized and the remaining intact cells were mechanically disrupted with a microfluidizer (Microfluidizer LM10, Microfluidics Corp., MA, 
USA, 1000 bar, 3 cycles). Extraction buffer was added to the lysate $(1: 1(v / v))$, urea added to this solution (6 M), and stirred for $30 \mathrm{~min}$ at RT. The lysate was then centrifuged for $50 \mathrm{~min}$ at $4{ }^{\circ} \mathrm{C}$ and $20379 \mathrm{rpm}$, the supernatant (S) separated from the pellet (P), and samples taken for SDS-PAGE analysis (Figure 3.8 A: P and S). The supernatant was incubated with $\mathrm{Ni}^{2+}$-NTA agarose beads (10 Ml, resuspended in washing buffer) and incubated for $3 \mathrm{~h}$ at $4{ }^{\circ} \mathrm{C}$ on a roller mixer to allow the protein to bind to the beads via the His $6^{-}$tag.

\section{Purification}

The protein-bead suspension was transferred to a column (Eco-column, $3 \times 13 \mathrm{~cm}$, BioRad Laboratory) and a sample for the SDS-PAGE taken from the flow through (Figure 3.8 A, FT). Washing buffer was added to re-suspended beads, incubated for $5 \mathrm{~min}$, and unspecifically bound proteins eluted. A sample for the SDS-PAGE was taken (Figure $3.8 \mathrm{~A}, \mathrm{~W}$ ) and the process repeated 3 times until the beads were re-suspended in $10 \mathrm{~mL}$ elution buffer, incubated for $10 \mathrm{~min}$, and the protein eluted from the beads. The elution process was repeated two times, samples E1-E3 and beads B were taken for SDS-PAGE analysis (Figure 3.8 A), and TCEP/DTT was added to a final concentration of $0.1 / 1 \mathrm{mM}(1 / 10 \mathrm{mM}$ stock). In this example, after performing the SDS-PAGE analysis, fractions with high protein concentration E1 and E2 were combined and thrombin (4 mg in $1 \mathrm{~mL}$ dialysis buffer) was added to the solution (100 $\mu \mathrm{L} / 5 \mathrm{~mL}$ elution volume). The protein solution was transferred to dialysis tubes with a molecular weight cutoff adjusted to the respective protein, tubes were transferred into dialysis buffer, stirred for $30 \mathrm{~min}$ at RT, and thrombin cleavage continued overnight at $4{ }^{\circ} \mathrm{C}$. Effective cleavage of the His ${ }_{6}$-tag was visible as a reduction of mass of the protein in the SDS-PAGE (Figure $3.8 \mathrm{~B}, \mathrm{bC}=$ before cleavage, $\mathrm{aC}=$ after cleavage).
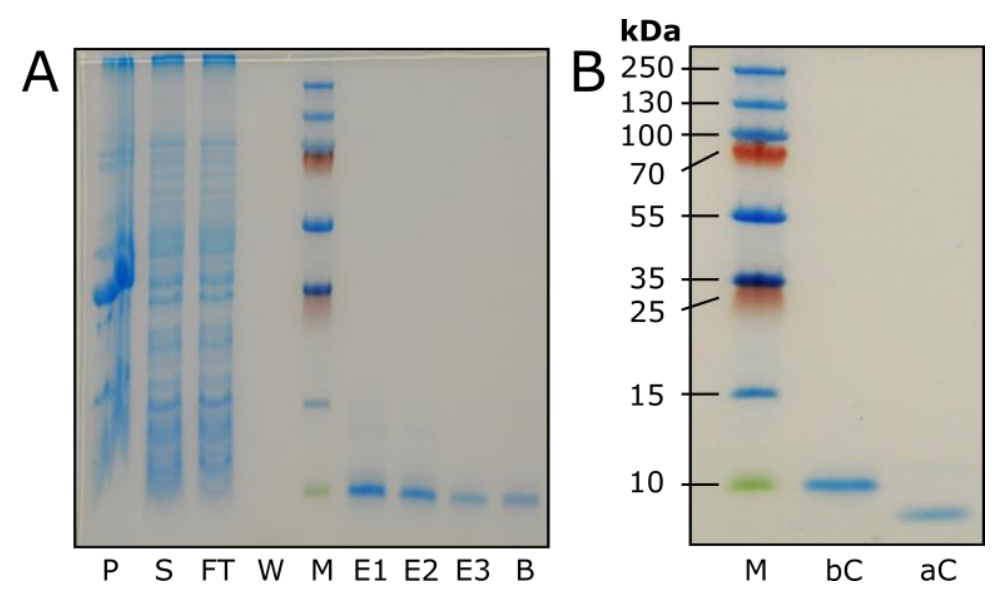

Figure 3.8 SDS-PAGEs of the isolation of Sb49-96 S79C. (A) Analysis of the cell lysis and $\mathrm{Ni}^{2+}$ affinity chromatography with P: pellet, S: supernatant, FT: flow through, W: wash, M: marker E1-3: elution fractions and 
B: beads. The elution fractions showed a strong band at $\sim 10 \mathrm{kDa}$. (B) Thrombin cleavage of the His 6 -tag of E1-3 with $\mathrm{M}$ : marker, bC: before cleavage and $\mathrm{aC}$ : after cleavage showed a decrease in molecular mass of $\sim 2 \mathrm{kDa}$.

The solution was transferred after thrombin cleavage to the ÄKTA purification system and further purified as described in Chapter 3.2.2 using a MonoS 5/50 GL column. The protein was eluted at a conductance of $28.88 \mathrm{mS} \mathrm{cm}^{-1}$ (Figure $3.9 \mathrm{~A}$ ) and elution fractions were analyzed with SDS-PAGE (Figure 3.9 B). Fractions 15-20 showed strong bands at $<10 \mathrm{kDa}$ and can be assigned to Sb49-96 S79C. Protein concentration was determined by means of UV/Vis-spectroscopy (NanoDrop200c, Thermo Fisher Scientific) and, if not directly used, shock frozen at $-80{ }^{\circ} \mathrm{C}$. A total protein amount of $10.803 \mathrm{mg}$ per $2.5 \mathrm{~L}$ medium was isolated.
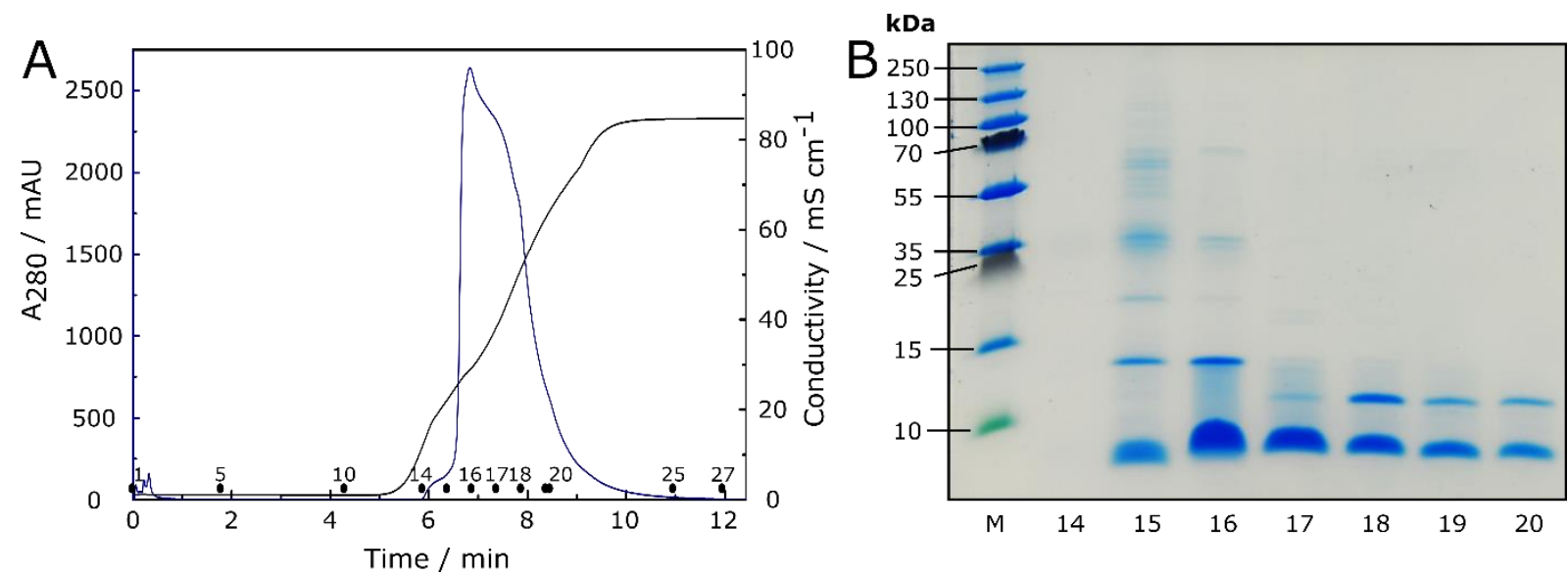

Figure 3.9 (A) Cation exchange chromatogram of Sb49-96 S79C using a MonoS 5/50 column. Sb49-96 S79C eluted between 25 and $60 \mathrm{mS} \mathrm{cm}^{-1}$ with a maximum $\mathrm{A}_{280}$ value at $28.88 \mathrm{mS} \mathrm{cm}^{-1}$. (B) SDS-PAGE of fraction 14-20 showed a strong band at $<10 \mathrm{kDa}$. Fraction 16 was chosen for further labeling experiments.

Protein labeling

Fraction 16 was used in this example for labeling with Atto488 maleimide under light-free conditions. Briefly, Atto488 maleimide (1 mg, $0.937 \mu \mathrm{mol}, 1.3$ Äq.) in $150 \mu \mathrm{L}$ ÄKTA buffer A was incubated with Sb49-96 S79C (4.068 mg, $0.721 \mu \mathrm{mol}, 1.0$ Äq.) for $2 \mathrm{~h}$ at RT. The protein was separated from excess fluorophores via size exclusion chromatography over a sephadex G-15 column (40 cm, $\varnothing=3 \mathrm{~cm})$ with ÄKTA buffer A and B $(0.65: 0.35(v / v))$. The result of the SDS-PAGE analysis is shown in Figure 3.10 with strong bands of Sb49-96-Atto488 at $<10 \mathrm{kDa}$. The degree of labeling $(D O L)$ was determined by means of UV/Vis-spectroscopy. First, the protein concentration $c_{\text {prot }}$ was calculated using Equation (3-1) with $A_{280}$ being the absorbance at $280 \mathrm{~nm}, \varepsilon_{\text {prot }}$ the extinction coefficient of the protein $\left(12490 \mathrm{M}^{-1} \mathrm{~cm}^{-1}\right)$, and $d$ the thickness of the cuvette. The product of $A_{\max }$ and $C F_{280}$ is a correction factor necessary due to the absorbance of the Atto488 label at $280 \mathrm{~nm}$ with $C F_{280}=0.09$. 


$$
c_{\text {prot }}=\frac{A_{280}-A_{\max } \cdot C F_{280}}{\epsilon_{\text {prot }} \cdot d}
$$

The mean protein concentration after labeling was $0.232 \mathrm{mg}$ per $\mathrm{mL}$. The $D O L$ was then calculated as the fraction of protein-label construct and overall protein concentration using Equation (3-2).

$$
D O L=\frac{c_{\text {label }}}{c_{\text {prot }}}=\frac{A_{\text {max }} \cdot \epsilon_{\text {prot }}}{\left(A_{280}-A_{\text {max }} \cdot C F_{280}\right) \cdot \epsilon_{\text {Atto } 488}}
$$

Here, $\varepsilon_{\text {Atto488 }}=9.0 \cdot 10^{4} \mathrm{M}^{-1} \mathrm{~cm}^{-1}$ is the extinction coefficient of the label at the absorption maximum. The mean $D O L$ was 0.534 at $2.729 \mathrm{mg}$ protein.

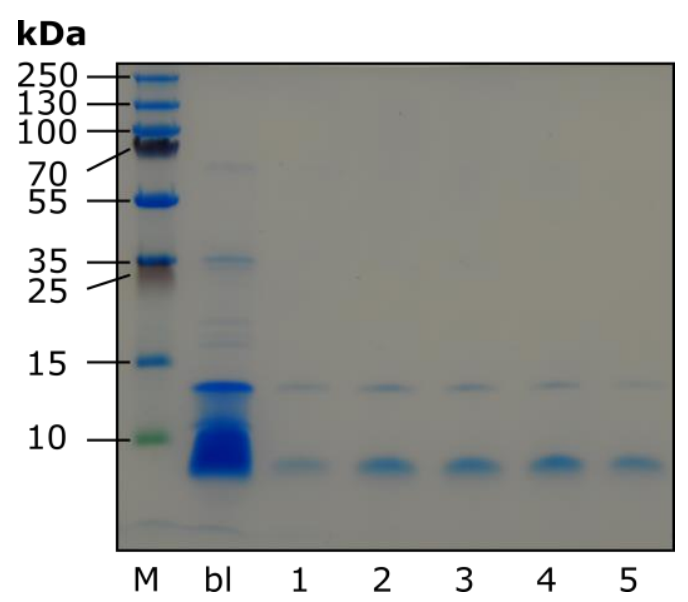

Figure 3.10 SDS-PAGE of Sb49-96 S79C-Atto488 with M: marker, bl: before labeling and 1-5 being collected fractions of the size exclusion chromatography.

\section{Synaptobrevin 2}

Due to its hydrophobic character, full length synaptobrevin 2 (12.691 kDa, pI =7.84, $\varepsilon=13980 \mathrm{M}^{-1} \mathrm{~cm}^{-1}$ ) had to be expressed and purified by adding detergents to the buffer solutions with compositions listed in Table 3-6. To change the used detergents prior to elution of the protein from the $\mathrm{Ni}^{2+}$-NTA agarose beads, beads were washed with 4 x $50 \mathrm{~mL}$ of washing buffer I and 2 x $50 \mathrm{~mL}$ of washing buffer II. Syb 2 eluted from a cation exchange column at a

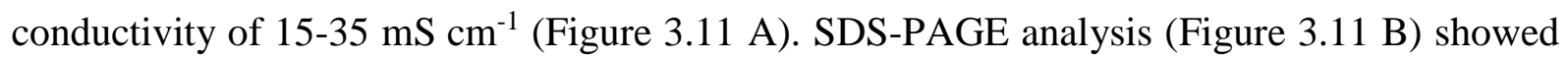
strong bands of fraction 5-10 at $\sim 13 \mathrm{kDa}$ that can be assigned to the protein. The overall yield was $10.3 \mathrm{mg}$ per $2.5 \mathrm{~L}$ main culture. 

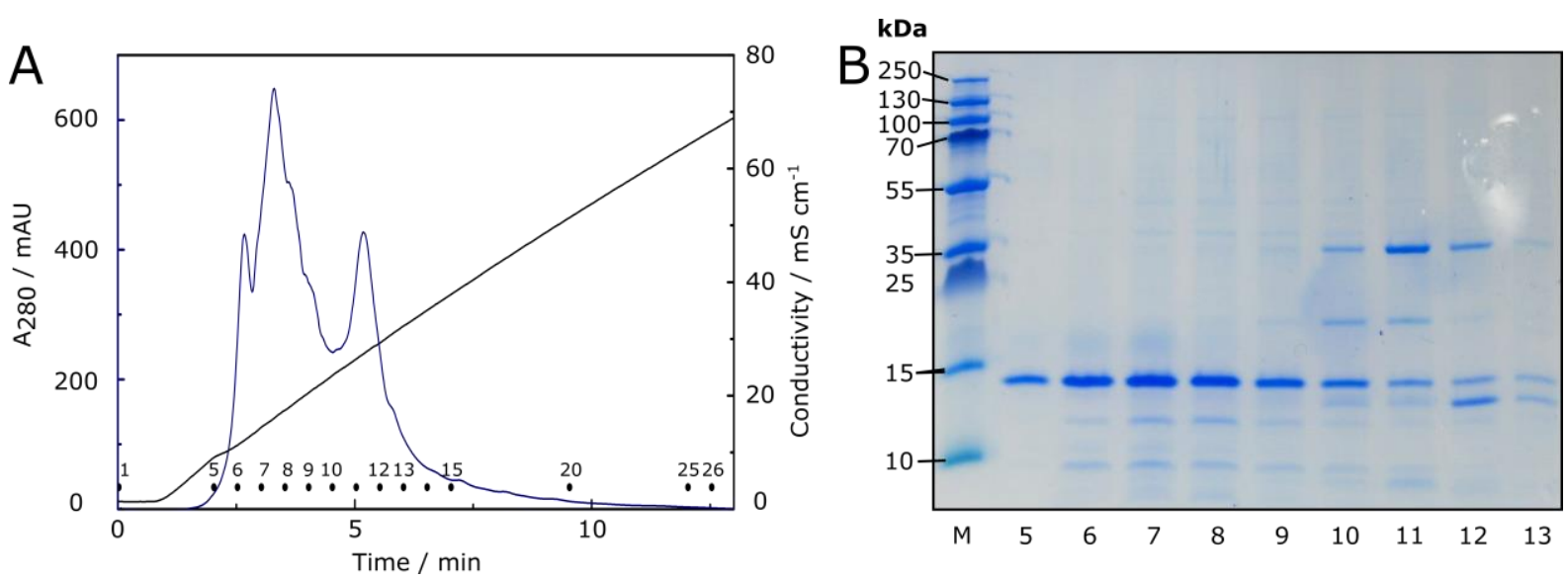

Figure 3.11 (A) Cation exchange chromatogram of synaptobrevin 2 (aa 1-116) using a MonoS 5/50 column. Synaptobrevin 2 eluted from $15-35 \mathrm{mS} \mathrm{cm}^{-1}$. (B) SDS-PAGE of fractions 5-13 showed strong bands at $\sim 13 \mathrm{kDa}$. 
Table 3-6 Buffer compositions for the isolation and purification of syb 2 (aa 1-116).

\begin{tabular}{|c|c|c|}
\hline & Chemical & Concentration \\
\hline Washing buffer I & $\begin{array}{l}\text { TRIS/HCl } \\
\mathrm{NaCl} \\
\text { Imidazole } \\
\text { Sodium cholate }\end{array}$ & $\begin{array}{l}20 \mathrm{mM}, \mathrm{pH}=7.4 \\
500 \mathrm{mM} \\
20 \mathrm{mM} \\
1 \%(w / v)\end{array}$ \\
\hline Washing buffer II & $\begin{array}{l}\text { TRIS/HCl } \\
\mathrm{NaCl} \\
\text { Imidazole } \\
\text { CHAPS }\end{array}$ & $\begin{array}{l}20 \mathrm{mM}, \mathrm{pH}=7.4 \\
500 \mathrm{mM} \\
20 \mathrm{mM} \\
1 \%(w / v)\end{array}$ \\
\hline Elution buffer & $\begin{array}{l}\text { TRIS/HCl } \\
\mathrm{NaCl} \\
\text { Imidazole } \\
\text { CHAPS } \\
\text { DTT }\end{array}$ & $\begin{array}{l}20 \mathrm{mM}, \mathrm{pH}=7.4 \\
500 \mathrm{mM} \\
400 \mathrm{mM} \\
1 \%(w / v) \\
1 \mathrm{mM}\end{array}$ \\
\hline Dialysis buffer & $\begin{array}{l}\mathrm{HEPES} / \mathrm{NaOH} \\
\mathrm{NaCl} \\
\text { EDTA } \\
\text { CHAPS } \\
\text { DTT }\end{array}$ & $\begin{array}{l}20 \mathrm{mM}, \mathrm{pH}=7.4 \\
150 \mathrm{mM} \\
1 \mathrm{mM} \\
1 \%(w / v) \\
1 \mathrm{mM}\end{array}$ \\
\hline ÄKTA buffer A & $\begin{array}{l}\text { HEPES/NaOH } \\
\text { EDTA } \\
\text { DTT } \\
\text { CHAPS }\end{array}$ & $\begin{array}{l}20 \mathrm{mM}, \mathrm{pH}=7.4 \\
1 \mathrm{mM} \\
1 \mathrm{mM} \\
1 \%(w / v)\end{array}$ \\
\hline ÄKTA buffer B & $\begin{array}{l}\mathrm{HEPES} / \mathrm{NaOH} \\
\mathrm{NaCl} \\
\text { EDTA } \\
\text { DTT } \\
\text { CHAPS }\end{array}$ & $\begin{array}{l}20 \mathrm{mM}, \mathrm{pH}=7.4 \\
1 \mathrm{M} \\
1 \mathrm{mM} \\
1 \mathrm{mM} \\
1 \%(w / v)\end{array}$ \\
\hline
\end{tabular}




\section{Syntaxin 1A}

Due to its high hydrophobic character, t-SNARE syx 1A (aa 183-288, $12.035 \mathrm{kDa}, \mathrm{pI}=5.90$, $\varepsilon=4470 \mathrm{M}^{-1} \mathrm{~cm}^{-1}$ ) had to be expressed and purified with slight modifications and buffer conditions shown in Table 3-7. Briefly, detergents like sodium cholate and CHAPS as well as urea were added to certain buffers. To avoid precipitation of protein during dialysis, urea concentration was decreased stepwise prior to dialysis with dilution buffer I $(1: 1 \mathrm{v} / \mathrm{v})$, and two different dialysis buffers used to slowly lower the salt concentration. Instead of ÄKTA buffer A, dilution buffer II was used to adjust the conductivity of the protein solution prior to IEC. Syx $1 \mathrm{~A}$ eluted at a conductivity of $30-40 \mathrm{mS} \mathrm{cm}^{-1}$ (Figure $3.12 \mathrm{~A}$ ) as a single peak from the anion exchange column. SDS-PAGE analysis (Figure 3.12 B) of ÄKTA fractions 8-13 showed strong bands at $\sim 12 \mathrm{kDa}$ that can be assigned to the protein. The overall yield was $23 \mathrm{mg}$ per $2.5 \mathrm{~L}$ main culture.
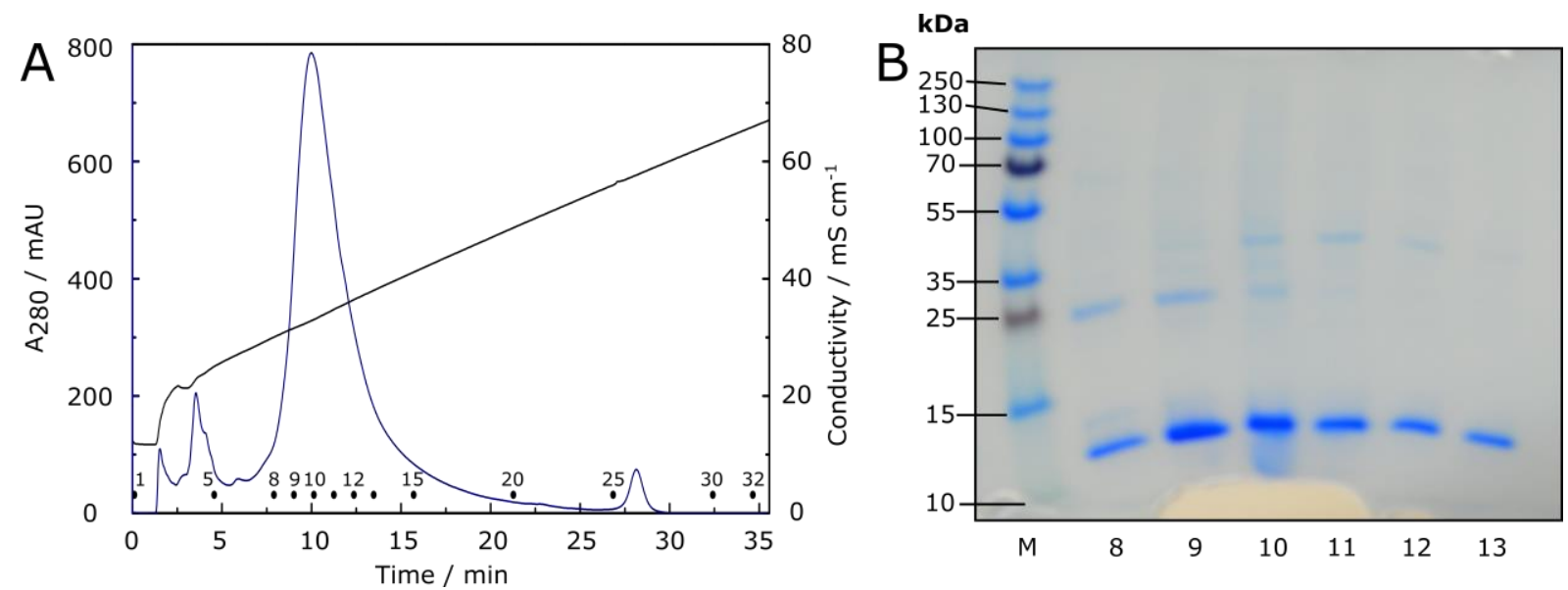

Figure 3.12 Anion exchange chromatogram of syntaxin 1A using a MonoQ 10/100 column. Syntaxin 1A eluted from $30-40 \mathrm{mS} \mathrm{cm}^{-1}$. (B) SDS-PAGE of fractions 8-13 showed a strong band at $\sim 12 \mathrm{kDa}$.

Table 3-7 Buffer compositions for the isolation and purification of syx 1A.

\begin{tabular}{l|l|l} 
& Chemical & Concentration \\
\hline \multirow{2}{*}{ Resuspension buffer } & TRIS/HCl & $20 \mathrm{mM}, \mathrm{pH}=7.4$ \\
$\mathrm{NaCl}$ & $500 \mathrm{mM}$ \\
Imidazole & $8 \mathrm{mM}$ \\
Extraction buffer & TRIS/HCl & $20 \mathrm{mM}, \mathrm{pH}=7.4$ \\
NaCl & $500 \mathrm{mM}$ \\
Imidazole & $8 \mathrm{mM}$
\end{tabular}




\begin{tabular}{|c|c|c|}
\hline & Sodium cholate & $10 \%(w / v)$ \\
\hline \multirow[t]{5}{*}{ Washing buffer } & TRIS/HCl & $20 \mathrm{mM}, \mathrm{pH}=7.4$ \\
\hline & $\mathrm{NaCl}$ & $500 \mathrm{mM}$ \\
\hline & Imidazole & $20 \mathrm{mM}$ \\
\hline & Sodium cholate & $3 \%(w / v)$ \\
\hline & Urea & $6 \mathrm{M}$ \\
\hline \multirow[t]{6}{*}{ Elution buffer } & TRIS/HCl & $20 \mathrm{mM}, \mathrm{pH}=7.4$ \\
\hline & $\mathrm{NaCl}$ & $500 \mathrm{mM}$ \\
\hline & Imidazole & $400 \mathrm{mM}$ \\
\hline & Sodium cholate & $3 \%(w / v)$ \\
\hline & Urea & $6 \mathrm{M}$ \\
\hline & DTT & $1 \mathrm{mM}$ \\
\hline \multirow[t]{4}{*}{ Dilution buffer I } & TRIS/HCl & $20 \mathrm{mM}, \mathrm{pH}=7.4$ \\
\hline & $\mathrm{NaCl}$ & $1 \mathrm{M}$ \\
\hline & Sodium cholate & $1.5 \%(w / v)$ \\
\hline & DTT & $1 \mathrm{mM}$ \\
\hline \multirow[t]{3}{*}{ Dilution buffer II } & TRIS/HCl & $20 \mathrm{mM}, \mathrm{pH}=7.4$ \\
\hline & DTT & $1 \mathrm{mM}$ \\
\hline & CHAPS & $1 \%(w / v)$ \\
\hline \multirow[t]{6}{*}{ Dialysis buffer I } & TRIS/HCl & $20 \mathrm{mM}, \mathrm{pH}=7.4$ \\
\hline & $\mathrm{NaCl}$ & $1 \mathrm{M}$ \\
\hline & Sodium cholate & $3 \%(w / v)$ \\
\hline & Urea & $2 \mathrm{M}$ \\
\hline & EDTA & $1 \mathrm{mM}$ \\
\hline & DTT & $1 \mathrm{mM}$ \\
\hline \multirow[t]{4}{*}{ Dialysis buffer II } & TRIS/HCl & $20 \mathrm{mM}, \mathrm{pH}=7.4$ \\
\hline & $\mathrm{NaCl}$ & $200 \mathrm{mM}$ \\
\hline & Sodium cholate & $3 \%(w / v)$ \\
\hline & EDTA & $1 \mathrm{mM}$ \\
\hline
\end{tabular}


ÄKTA buffer A

ÄKTA buffer B

\begin{tabular}{l|l} 
DTT & $1 \mathrm{mM}$ \\
TRIS/HCl & $20 \mathrm{~mm}, \mathrm{pH}=7.4$ \\
$\mathrm{NaCl}$ & $250 \mathrm{~mm}$ \\
EDTA & $1 \mathrm{~mm}$ \\
DTT & $1 \mathrm{mM}$ \\
CHAPS & $1 \%(w / v)$ \\
TRIS/HCl & $20 \mathrm{mM}, \mathrm{pH}=7.4$ \\
NaCl & $1 \mathrm{~mm}$ \\
EDTA & $1 \mathrm{~mm}$ \\
DTT & $1 \mathrm{~mm}$ \\
CHAPS & $1 \%(w / v)$
\end{tabular}

\section{SNAP25a}

Buffer compositions changed for the purification of full length SNAP25a (aa 1-206, 23.207 $\left.\mathrm{kDa}, \mathrm{pI}=4.74, \varepsilon=7240 \mathrm{M}^{-1} \mathrm{~cm}^{-1}\right)$ are shown in Table 3-8. The protein eluted from an anion exchange column as a single peak at a conductivity of $14-18 \mathrm{mS} \mathrm{cm}^{-1}$ (Figure $3.13 \mathrm{~A}$ ). Thrombin eluted at lower conductivities from the column. SDS-PAGE analysis (Figure 3.13 B) showed strong bands of fraction $36-44$ at $\sim 23 \mathrm{kDa}$ that can be assigned to the protein. The overall yield was $4.4 \mathrm{mg}$ per $2.5 \mathrm{~mL}$ main culture.
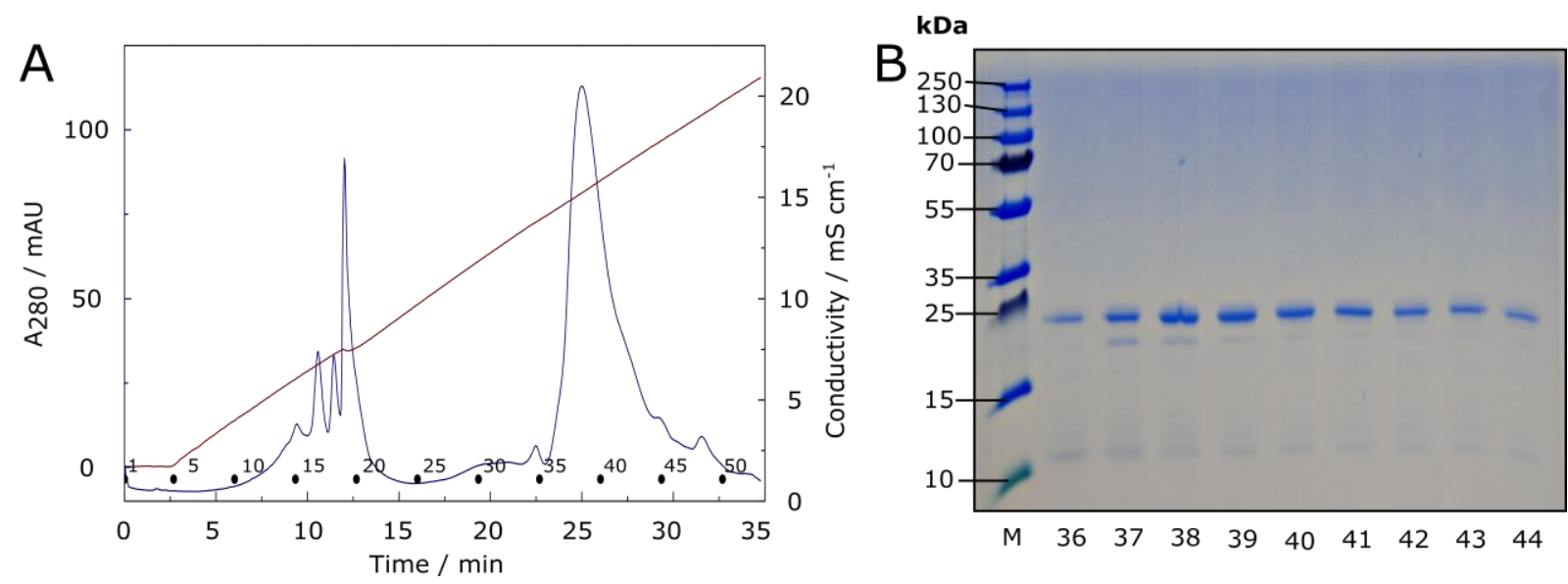

Figure 3.13 Anion exchange chromatogram of SNAP25a using a MonoQ 10/100 column. SNAP25a eluted from $14-18 \mathrm{mS} \mathrm{cm}^{-1}$. (B) SDS-PAGE of fractions 36-44 showed a strong band at $\sim 23 \mathrm{kDa}$. 
Table 3-8 Modified buffer compositions for the isolation and purification of SNAP25a.

\begin{tabular}{l|l|l} 
& Chemical & Concentration \\
\hline Dialysis buffer & TRIS/HCl & $20 \mathrm{mM}, \mathrm{pH}=7.4$ \\
$\mathrm{NaCl}$ & $50 \mathrm{~mm}$ \\
EDTA & $1 \mathrm{mM}$ \\
DTT & $1 \mathrm{mM}$ \\
ÄKTA buffer A & TRIS/HCl & $20 \mathrm{~mm}, \mathrm{pH}=7.4$ \\
EDTA & $1 \mathrm{mM}$ \\
ÄKTA buffer B & DTT & $1 \mathrm{mM}$ \\
& TRIS/HCl & $20 \mathrm{mM}, \mathrm{pH}=7.4$ \\
NaCl & $1 \mathrm{M}$ \\
EDTA & $1 \mathrm{mM}$ \\
& DTT & $1 \mathrm{mM}$
\end{tabular}

\section{$\Delta \mathbf{N} 49$-complex}

According to Pobbati et al. the $\Delta$ N49-complex was assembled from the three proteins SNAP25a, syx 1A and Sb49-96. ${ }^{[51]}$ For this, 1.5 Äq. Sb49-96 and 1.5 Äq. SNAP25a were each suspended in $1 \%$ CHAPS ( $w / v)$, Sb49-96 mixed with syx 1A (1.0 Äq.), and incubated for $30 \mathrm{~min}$ at $4{ }^{\circ} \mathrm{C}$ on a roller mixer. The solution was added to SNAP25a and incubated overnight at $4{ }^{\circ} \mathrm{C}$ on a roller mixer. $1 \%$ CHAPS ( $w / v$ ) was added to ÄKTA buffer A and B (Table 3-9). The $\Delta$ N49-complex $\left(40.885 \mathrm{kDa}, \varepsilon=24200 \mathrm{M}^{-1} \mathrm{~cm}^{-1}\right)$ was eluted from the anion exchange column between 20 and $35 \mathrm{mS} \mathrm{cm}^{-1}$, while remaining SNAP25a eluted at lower conductivities (Figure 3.14 A). Excess Sb49-96 did not bind to the stationary phase due to its pI of 9.16. SDS-PAGE analysis of fraction 15-17 showed strong bands for all three assembled proteins: SNAP25a at $\sim 23 \mathrm{kDa}$, syx 1A at $\sim 12 \mathrm{kDa}$, and Sb49-96 at $<10 \mathrm{kDa}$ (Figure $3.14 \mathrm{~B}$ ). The overall field was $39 \mathrm{nmol}$ of acceptor complex. 

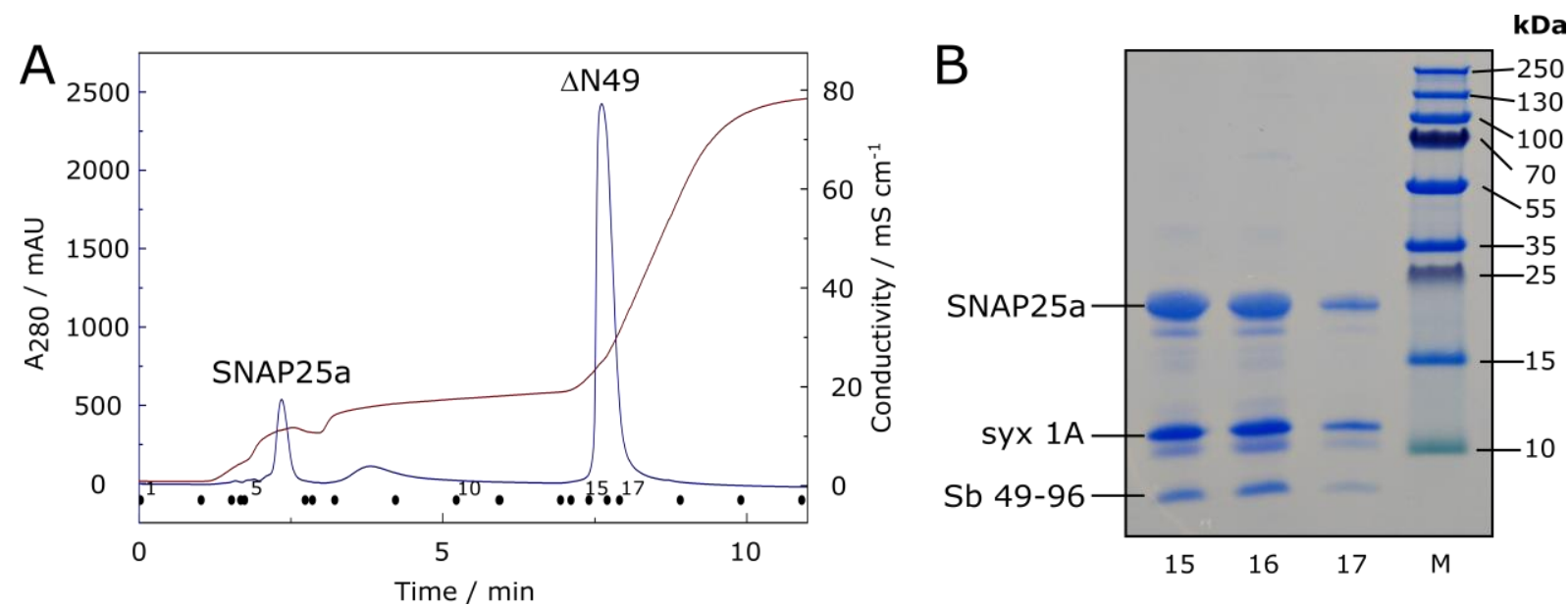

Figure 3.14 Anion exchange chromatogram of $\Delta$ N49-complex using a MonoQ 5/50 column. Remaining SNAP25a eluted at $\sim 15 \mathrm{mS} \mathrm{cm}^{-1}$, the $\Delta \mathrm{N} 49$-complex eluted between 20 and $35 \mathrm{mS} \mathrm{cm}^{-1}$. (B) SDS-PAGE of fractions 15-17 showed strong bands at $\sim 23 \mathrm{kDa}, \sim 13 \mathrm{kDa}$ and $<10 \mathrm{kDa}$ belong to SNAP25a, syntaxin 1A and Sb49-96 respectively.

Table 3-9 Modified buffer compositions for the ÄKTA purification of $\Delta$ N49-complex.

\begin{tabular}{l|l|l} 
& Chemical & Concentration \\
\hline \multirow{2}{*}{ ATTA buffer A } & HEPES/NaOH & $20 \mathrm{mM}, \mathrm{pH}=7.4$ \\
EDTA & $1 \mathrm{mM}$ \\
DTT & $1 \mathrm{mM}$ \\
CHKTA buffer B & HEPES/NaOH & $20(w / v)$ \\
NaCl & $1 \mathrm{mM}, \mathrm{pH}=7.4$ \\
EDTA & $1 \mathrm{mM}$ \\
DTT & $1 \mathrm{mM}$ \\
CHAPS & $1 \%(w / v)$
\end{tabular}

\section{$\Delta$ N49-Atto488-complex}

The $\Delta$ N49-Atto488-complex was assembled and purified as the $\Delta$ N49-complex using labeled Sb49-96-Atto488 under exclusion of light during all preparation steps. The $\Delta$ N49-Atto $488-$ complex $\left(41.704 \mathrm{kDa}, \varepsilon=24200 \mathrm{M}^{-1} \mathrm{~cm}^{-1}\right)$ eluted from the anion exchange column between 20 and $35 \mathrm{mS} \mathrm{cm}^{-1}$ while remaining SNAP25a eluted at lower conductivities (Figure $3.15 \mathrm{~A}$ ). Excess of Sb49-96-Atto488 did not bind to the stationary phase due to its pI of 9.16. SDS-PAGE analysis of fractions 12-15 showed strong bands for all three assembled proteins: SNAP25a at $23 \mathrm{kDa}$, syx $1 \mathrm{~A}$ at $\sim 12 \mathrm{kDa}$ and Sb49-96-Atto488 at < $10 \mathrm{kDa}$ (Figure 3.15). 

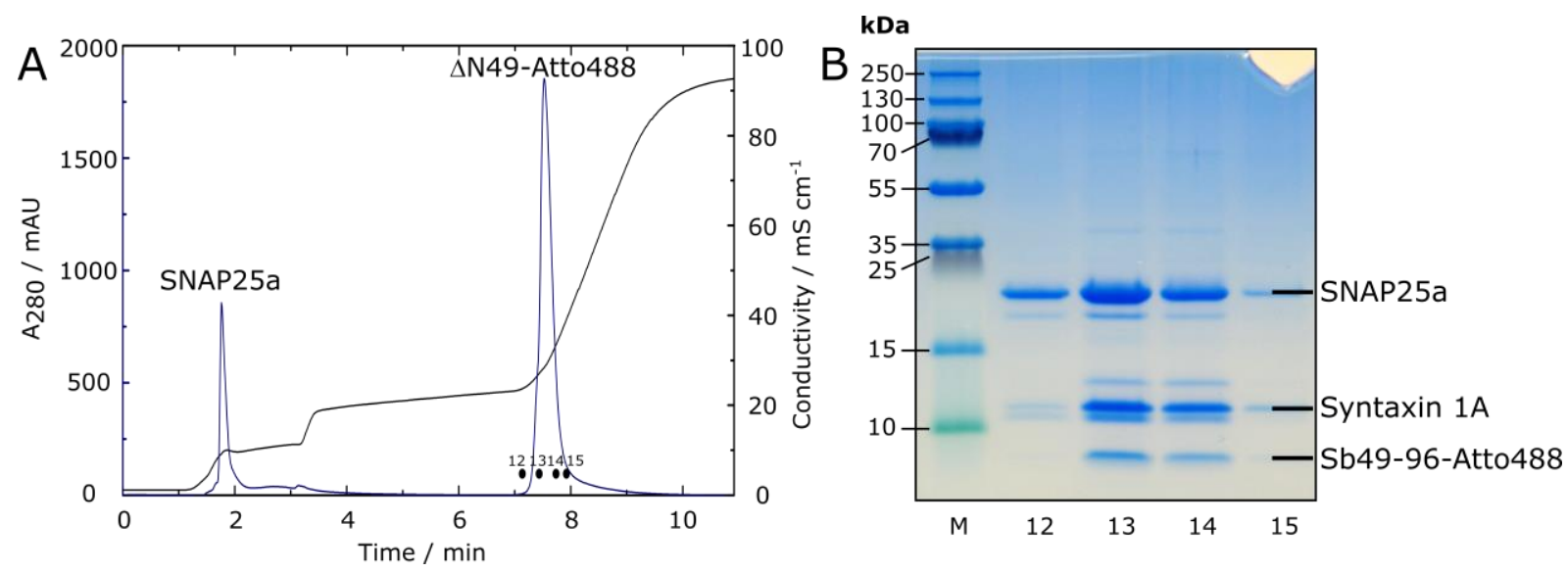

Figure 3.15 Anion exchange chromatogram of $\Delta$ N49-Atto488 using a MonoQ 5/50 column. Remaining SNAP25a eluted at $\sim 15 \mathrm{mS} \mathrm{cm}^{-1}$, the $\Delta \mathrm{N} 49$-Atto488-complex eluted between 20 and $35 \mathrm{mS} \mathrm{cm}^{-1}$. (B) SDS-PAGE of fractions 12-15 showed strong bands at $\sim 23 \mathrm{kDa}, \sim 13 \mathrm{kDa}$ and $<10 \mathrm{kDa}$ belonging to SNAP25a, syntaxin 1A and Sb49-96-Atto488, respectively.

Protein concentration and DOL were determined using Equation (3-1) and Equation (3-2), respectively. Figure 3.16 shows the absorbance spectra for Sb49-96-Atto488 (A) and $\Delta \mathrm{N} 49-$ Atto488 (B) with a visible increase in protein absorption at $280 \mathrm{~nm}$ after formation of the acceptor complex. The overall yield was $0.78 \mathrm{mg}$ protein with a $D O L$ of $35 \%$.
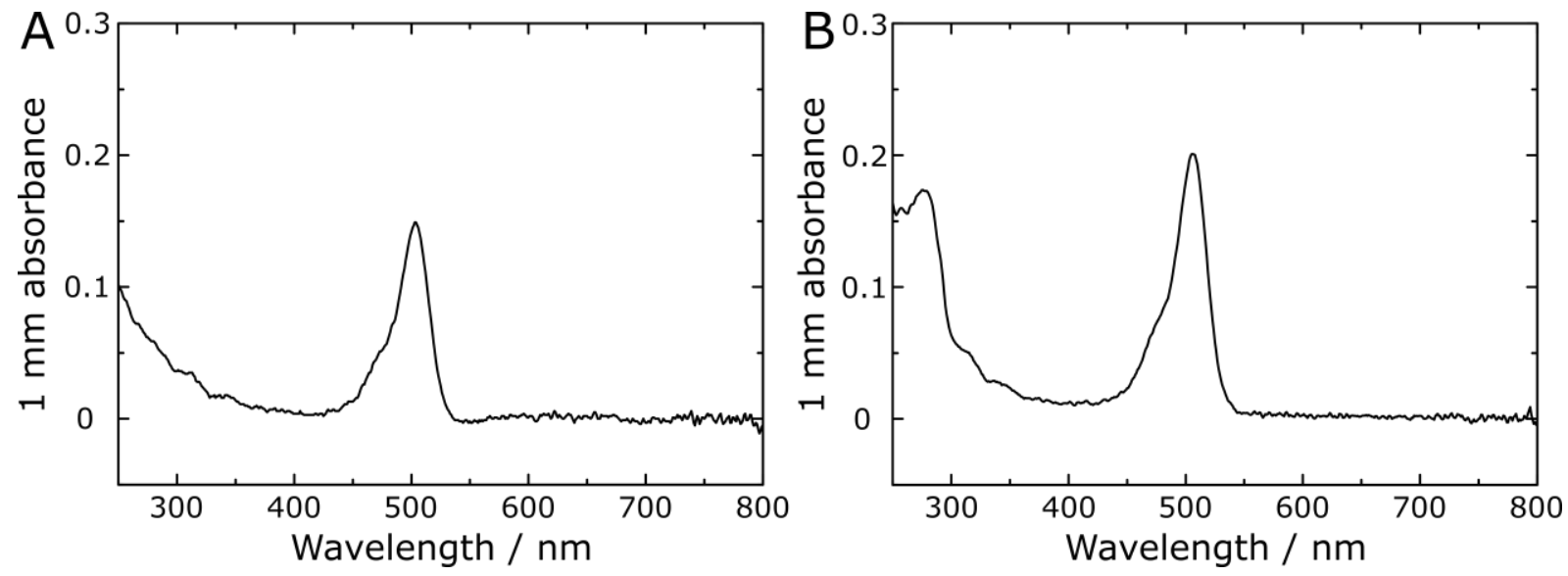

Figure 3.16 Absorbance spectra of (A) Sb49-96-Atto488 and (B) $\Delta$ N49-Atto488 show high absorbance at $\sim 300 \mathrm{~nm}$ and $\sim 500 \mathrm{~nm}$ belong to the protein residues and the Atto488-label, respectively.

\subsubsection{SNARE protein reconstitution into liposomes}

SNARE proteins and protein constructs were reconstituted into liposomes using a co-micellization procedure with $n$-octyl- $\beta$-D-glycoside ( $n$-OG) and subsequent detergent removal via rapid dilution as described previously. ${ }^{[51]}$

All following indications of quantity are for single-vesicle fusion experiments. Prior to SNARE reconstitution, lipids (DOPC/POPE/POPS/cholesterol; 5/2/1/2 (n/n), 0.465 $\mathrm{mg}$ total) and any 
lipid coupled fluorophore were dissolved in a test tube in $750 \mu \mathrm{l}$ chloroform and the solvent removed at $30{ }^{\circ} \mathrm{C}$ under a constant flow of Nitrogen. Afterwards, the remaining solvent was removed by placing the test tubes in vacuo for $2 \mathrm{~h}$ at $\mathrm{RT}$ and kept at $4{ }^{\circ} \mathrm{C}$ until use for up to 0.5 years. Buffers were degassed in vacuo for $30 \mathrm{~min}$ followed by DTT addition and, including all other solutions, prepared freshly and kept for up to one week under protection from light.

\section{Proteo-SUVs}

Prior to protein addition, lipid films were rehydrated with $50 \mu \mathrm{l}$ of SNARE buffer A (20 mM HEPES, $100 \mathrm{~mm} \mathrm{KCl}, 1 \mathrm{~mm}$ DTT, $217 \mathrm{mOsm}, \mathrm{pH}$ 7.4) and $n$-OG for $30 \mathrm{~min}$ at $0{ }^{\circ} \mathrm{C}$. Gently mixing with unfrozen protein solution results in a final $n$-OG concentration of $75 \mathrm{~mm}$ and a nominal protein to lipid ratio $(\mathrm{p} / \mathrm{l})$ of 1:500. The solution was incubated for $45 \mathrm{~min}$ at $0{ }^{\circ} \mathrm{C}$ and proteo-SUVs were formed by rapidly diluting the mixture below the critical micelle concentration of $n$-OG combined with size exclusion chromatography (illustra NAP-10 G25 column) in SNARE buffer A. To remove remaining detergent and salts from the solution, the mixture was concentrated to $\sim 50 \mu \mathrm{L}$ using a Concentrator 5301 (Eppendorf, Hamburg, GER) and a second size exclusion chromatography step was performed with ultrapure water. The solution was again concentrated to $\sim 50 \mu \mathrm{L}$ and for the preparation of giant unilamellar vesicles (GUVs) SUVs were placed in droplets of $2 \mu \mathrm{L}$ on indium tin oxide (ITO) slides while for the preparation of LUVs the solution was transferred in a round bottom flask. Both solutions were dried in a desiccator over a saturated $\mathrm{NaCl}$ solution overnight at $4{ }^{\circ} \mathrm{C}$.

\section{Content labeled proteo-LUVs}

$500 \mu \mathrm{L}$ of a solution of SRB in SNARE buffer A (43 mM, $255 \mathrm{mOsM}$, adjusted to $\mathrm{pH} 7.4$ ) were added to the dried proteo-lipid film and incubated for $30 \mathrm{~min}$ at RT. Subsequently, the suspended lipid film was extruded through a $400 \mathrm{~nm}$ polycarbonate membrane for 31 times using a mini extruder (LiposoFast-Basic, Avestin, Otawa, Ontario, Canada). Content labeled proteo-LUVs were separated from the bulk SRB solution by size exclusion chromatography (illustra NAP-25 G25 column) in SNARE buffer B (20 mM HEPES, $121 \mathrm{mM} \mathrm{KCl,} 1 \mathrm{~mm}$ dithiothreitol, $255 \mathrm{mOsM}, \mathrm{pH} 7.4$ ) to obtain LUVs with a mean diameter of $240 \pm 100 \mathrm{~nm}$ and an effective p/l ratio of 1:600 as determined previously. ${ }^{[61]}$ Successful incorporation of SRB into LUVs was tested by UV/Vis spectroscopy (Chapter 4.1). 


\section{Bulk fusion assay}

Functional reconstitution of v-SNAREs into LUVs and t-SNAREs into SUVs was tested by bulk content release experiments. Fusion of SRB filled LUVs with $\triangle$ N49 containing SUVs leads to the dequenching of SRB fluorescence and is recorded as a function of time. Briefly, $10 \mathrm{nmol}$ of LUV-lipid material is mixed with $10 \mathrm{nmol}$ of SUV-lipid material in a total volume of $750 \mu \mathrm{L}$ at $22{ }^{\circ} \mathrm{C}$. SRB fluorescence is excited at $\lambda_{\text {ex }}=560 \mathrm{~nm}$ and fluorescence recorded at $\lambda_{\mathrm{em}}=590$ for up to $30 \mathrm{~min}$. To induce vesicle lysis for a complete dequenching of SRB Triton $\mathrm{X}-100$ was added at the end of the recording time and final fluorescence intensity used for normalization.

\section{Proteo-GUVs}

ITO slides with dried proteo-lipid films were assembled into a chamber (Figure 3.17) and filled with sucrose solution $(1.6 \mathrm{~mL}, \sim 0.25 \mathrm{M}, 255 \mathrm{mOsM}$ ). Using a frequency generator (Agilent 32220A, Agilent Technologies) a sinusoidal voltage was stepwise increased $(0.01 \mathrm{~V}-0.2 \mathrm{~V}$, $0.01 \mathrm{~V} / \mathrm{min} ; 0.2 \mathrm{~V}-1.6 \mathrm{~V}, 0.1 \mathrm{~V} / \mathrm{min}$ ) and kept constant for up to $3 \mathrm{~h}$ at RT and $12 \mathrm{~Hz}$. The GUV solution was separated into 3 fractions and each fraction was analyzed for its purity and GUV density by means of fluorescence microscopy. The GUV-formation resulted in an effective $\mathrm{p} / 1$ ratio of 1:1900 as described in Chapter 4.2.

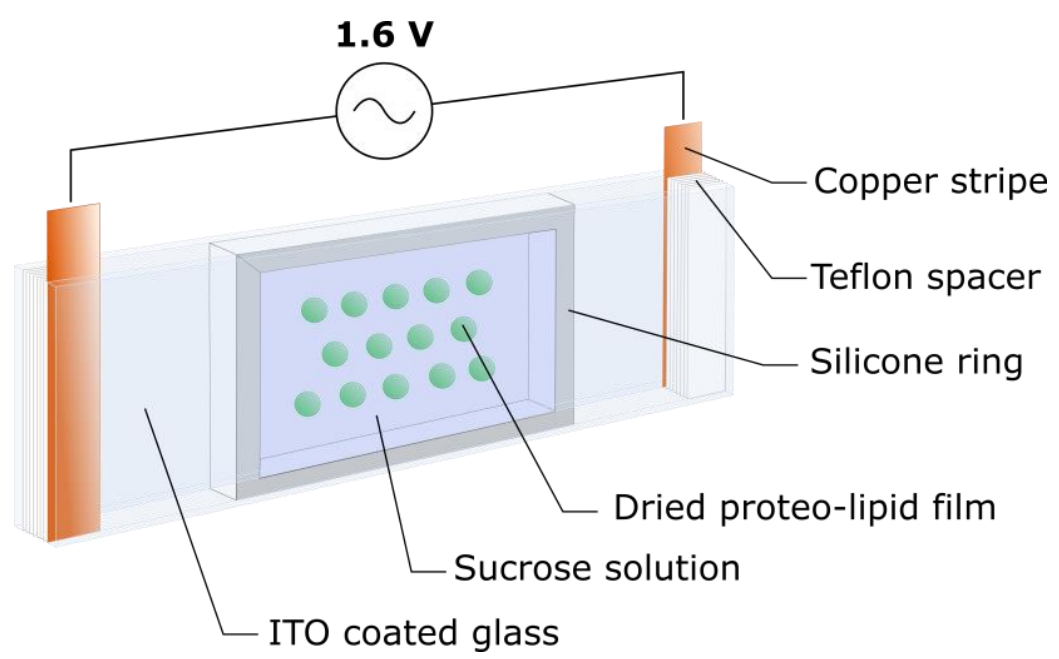

Figure 3.17 Schematic illustration of the electroformation chamber used in this work. A sinusoidal voltage is stepwise increased up to $1.6 \mathrm{~V}$ and kept constant for a total of $3 \mathrm{~h}$ at a frequency of $12 \mathrm{~Hz}$.

\subsubsection{Pore-spanning membranes (PSMs)}

Pore-spanning membranes (PSMs) were obtained by spreading giant unilamellar vesicles (GUVs, see Chapter 3.2.4) on top of functionalized porous substrates with open or closed holes and varying in their pore diameter (Figure 3.18) as schematically depicted in Figure 3.19. 
Substrates with open holes (Figure 3.18 A and B) had a pore diameter of 1.2 / $5 \mu \mathrm{m}$, a porosity of $35-40 \%$, a hexagonal pore arrangement, and were purchased from Aquamarijn B. V. (Zutphen, The Netherlands). The porous pattern with a thickness of $800 \mathrm{~nm}$ was stabilized by a $1 \mathrm{~mm}$ wide frame. Substrates with closed holes (Figure $3.18 \mathrm{C}$ and D) had a pore diameter of $3.5 \mu \mathrm{m}$, a pore depth of $250 \mathrm{~nm}$, a porosity of $20 \%$ with a squared arrangement, and were purchased from caesar (center of advanced European studies and research, Bonn, Germany). All substrates had an overall size of $5 \times 5 \mathrm{~mm}$ and were prepared using a photolithographic process.
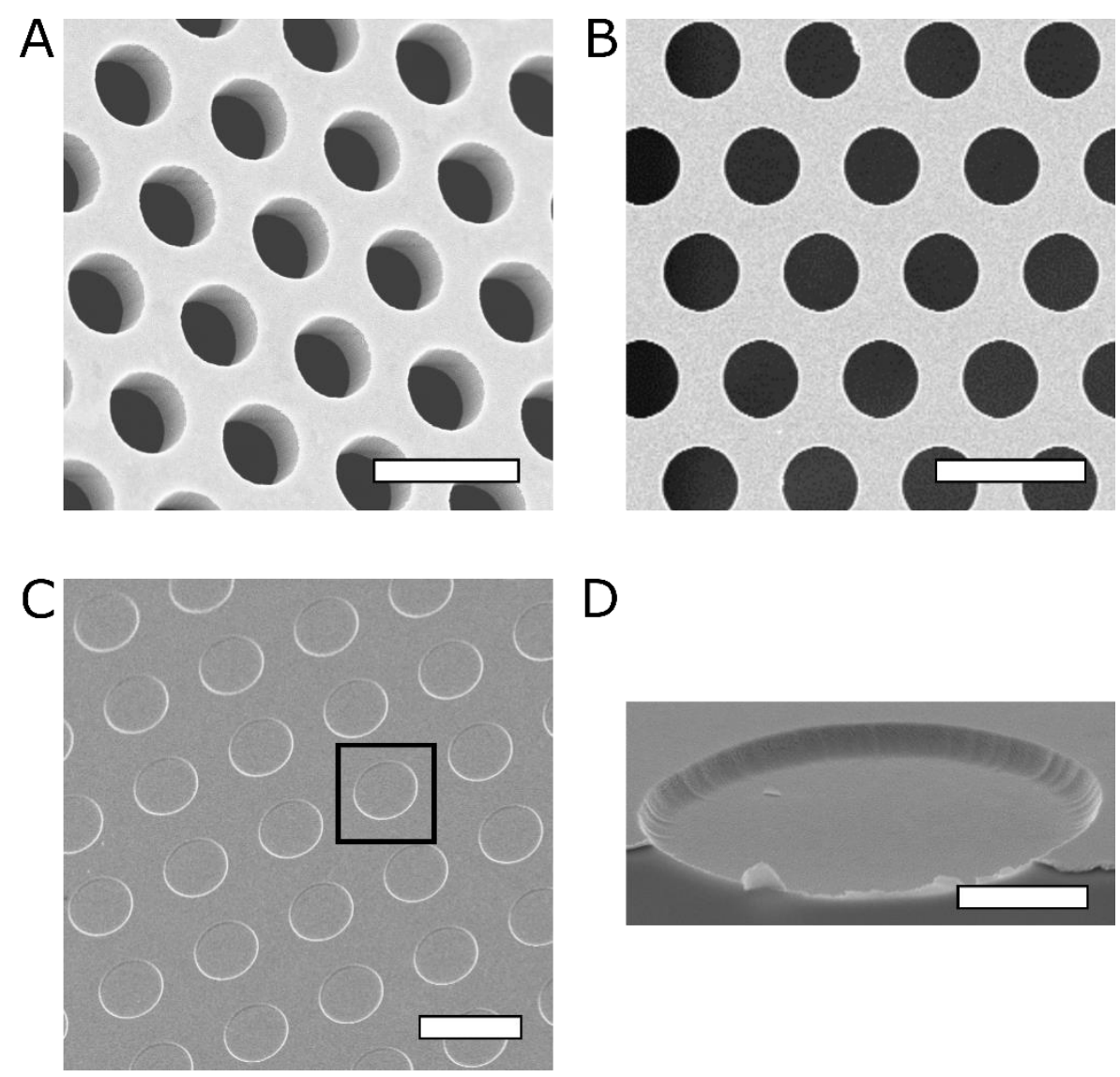

Figure 3.18 Scanning electron micrographs of porous substrates used to prepare pore spanning membranes. (A) Hexagonal pore array with pore diameters of $1.2 \mu \mathrm{m}$ used for single-vesicle fusion experiments. Scale bar $2 \mu \mathrm{m}$. (B) Hexagonal pore array with pore diameters of $5 \mu \mathrm{m}$ used for FRAP-experiments. Scale bar $=10 \mu \mathrm{m}(\mathrm{C})$ Squared pore array with closed compartments with pore diameters of $3.5 \mu \mathrm{m}$ and pore depths of $250 \mathrm{~nm}$ used to prepare PSMs with closed compartments. Scale bar $5 \mu \mathrm{m}$. (D) Zoom in of C coated with $40 \mathrm{~nm}$ gold. Scale bar $=1 \mu \mathrm{m}$.

Porous substrates were cleaned with ethanol, dried with nitrogen gas, and further cleaned with a combination of oxygen and argon plasma (50/50, 0.2 mbar, $30 \mathrm{~s}$, Zepto plasma cleaner, Diener Electronic, Ebbhausen, Germany). The cleaned surface was sputter coated under argon atmosphere with a thin titanium layer $(40 \mathrm{~mA}, 0.4$ mbar, $20 \mathrm{~s}$, Cressington Sputter Coater 
108auto, Watford, UK). Under ambient conditions the titanium layer quickly oxidizes to titanium dioxide which serves as an adhesion promoter for the gold layer applied in the following step. Approximately $40 \mathrm{~nm}$ of gold was orthogonally applied on top of the pore rim by means of thermal evaporation under vacuum $\left(\sim 6 \cdot 10^{-6} \mathrm{mbar}, \sim 60 \mathrm{~A}, 0.3-0.4 \mathrm{~nm} \mathrm{~s}^{-1}\right.$, MED020 coating system, Bal-Tec, Balzers, Lichtenstein). Closed substrates are covered completely with gold due to the low pore depth and the beveled pore edge (Figure 3.18 D). Freshly coated substrates were functionalized with a self-assembled monolayer (SAM) overnight at $8{ }^{\circ} \mathrm{C}$ in a $1 \mathrm{mM} n$-propanolic 6-mercapto-1-hexanol solution leading to a hydrophilic surface. The substrates were kept up to 2 months and thoroughly rinsed with ethanol and water before fixing them in the measuring chamber filled with iso-osmolar buffer in respect to the used GUV solution (SNARE buffer B for single-vesicle experiments). To form PSMs, $10-15 \mu \mathrm{L}$ of GUV solution were pipetted on top of the substrates and incubated for $\sim 30 \mathrm{~min}$. GUVs adhere to the hydrophilic surface and increase of contact area subsequently results in the spontaneous rupture of GUVs and the formation of PSMs. Prior to further measurements, excess lipid material was washed away from the measurement chamber. Since gold surfaces quench fluorescence emission in close distances of up to $15 \mathrm{~nm}$ only the freestanding part of the PSM (f-PSM) is visible under the fluorescence microscope. ${ }^{[93]}$

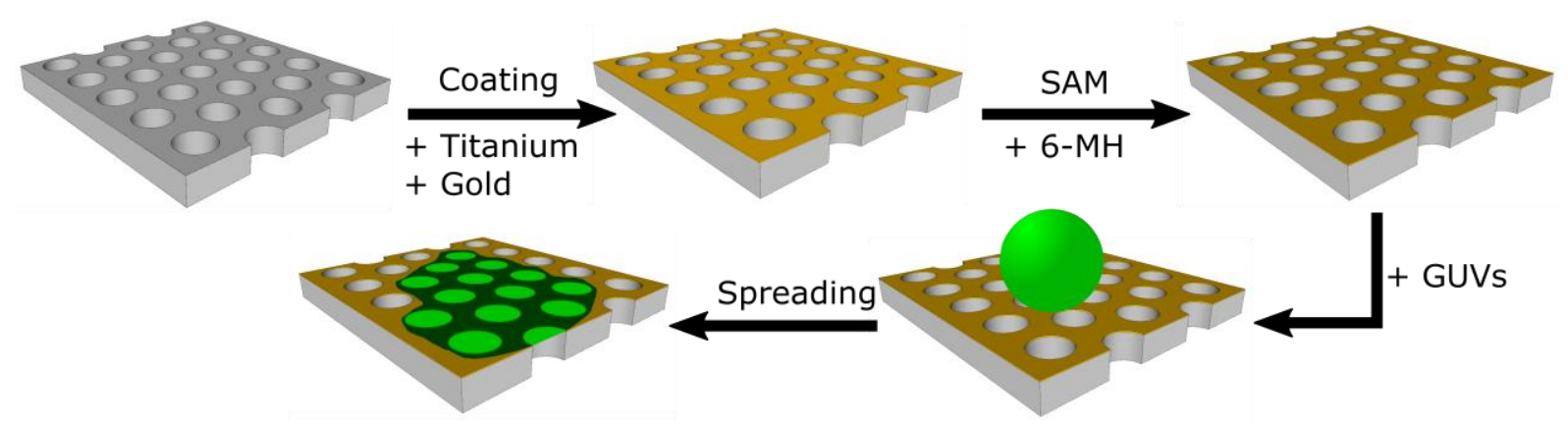

Figure 3.19 Schematic illustration of the preparation of pore-spanning membranes (PSMs). The porous $\mathrm{SiO}_{2} / \mathrm{Si}_{3} \mathrm{~N}_{4}$ substrate is orthogonally coated with a thin layer of titanium/titanium dioxide and a $\sim 40 \mathrm{~nm}$ thick gold layer. Addition of a $1 \mathrm{mM} n$-propanolic 6-mercapto-1-hexanol solution leads to the formation of a self-assembled monolayer (SAM) that enables the spreading of giant unilamellar vesicles (GUVs) on top of the substrate to form PSMs.

\subsection{Biophysical methods}

\subsubsection{Epifluorescence microscopy}

Epifluorescence microscopy is based on the absorption of light from fluorescent probes followed by the spontaneous emission of fluorescence of a higher wavelength. This so called Stokes-shift is used to specifically detect only the emitted light and thus to visualize biological samples. Different fluorescence dyes can be excited individually by adjusting the excitation 
wavelength of the white light source enabling the detection of several molecules inside the same sample. In this thesis, epifluorescence microscopy was used to quantify the reconstitution efficiency of the t-SNARE acceptor complex $\triangle \mathrm{N} 49$ into GUVs.

\section{Reconstitution efficiency of $\Delta$ N49-Atto488 in GUVs}

The reconstitution efficiency $R$ of the fusion active $\Delta$ N49-complex was determined using the labeled $\Delta \mathrm{N} 49$-Atto488-complex following a procedure reported by Aimon et al. ${ }^{[94]}$ with slight modifications. ${ }^{[95]}$

The evaluation is based on the direct correlation between peak membrane fluorescence intensity (Figure $3.20 \mathrm{~B}$ ) and the respective fluorophore concentration in the membrane. Quantifying membrane intensities of GUVs with known Atto488 DPPE concentrations and fitting the data with a weighted linear regression leads to a calibration factor $M_{\text {ref. }}$. Peak membrane fluorescence intensities of unknown $\Delta \mathrm{N} 49$-Atto488 concentration $I_{\mathrm{P}}$ then yield the reconstitution efficiency using Equation (3-3)

$$
R=\frac{I_{\mathrm{P}}}{M_{\mathrm{ref}} \cdot D O L \cdot 100 \cdot c_{0}}
$$

with $c_{0}$ ' being the nominal protein concentration of $0.2 \mathrm{~mol} / \mathrm{L}$ and $D O L$ the degree of labeling of the protein (see Chapter 3.2.3).

Experimental procedure

Proteo-GUVs as well as GUVs with known Atto488 DPPE concentration were prepared as described in Chapter 3.2.4 with a sucrose concentration of $0.2 \mathrm{~mol} / \mathrm{L} .10-20 \mu \mathrm{L}$ of GUV solution were added to a petri dish filled with $0.2 \mathrm{M}$ glucose solution. The slight difference in density between sucrose and glucose leads to the GUVs floating stable inside the solution. Thus, fluorescence imaging could be performed without any contact area between membrane and substrate that might lead to deformation of the GUV or protein aggregation on the surface. GUVs were imaged using the epifluorescence microscope BX 51 (Olympus, Tokyo, Japan) equipped with a water immersion objective (40xW / NA 0.8, Carl Zeiss, Jena, Germany), a mercury vapor lamp (U-HGLPS, Olympus, Tokyo, Japan), and an EMCCD-camera (iXon, DV885KCS-VP, 1004x1002 pixel, Andor technology, Belfast, United Kingdom). The mercury lamp was switched on $1 \mathrm{~h}$ before starting the measurements to ensure stable illumination. For quantitative comparability of fluorescence intensities between measuring days all images were 
acquired in the equatorial plane of the GUV (see Figure 3.20 A) using identical settings listed in Table 3-10.

Table 3-10 Microscopy settings to quantify the reconstitution efficiency of $\Delta$ N49-complex in GUVs.

\begin{tabular}{l|l} 
& User settings \\
\hline illumination time & $0.5 \mathrm{~s}$ \\
electron multiplier gain & 20 \\
mercury illumination level & 12 \\
bit depth & 16
\end{tabular}

Background corrected membrane peak fluorescence intensities (see Figure $3.20 \mathrm{~B}$ ) were extracted using a custom written MATLAB script written by a former coworker Markus Schön.
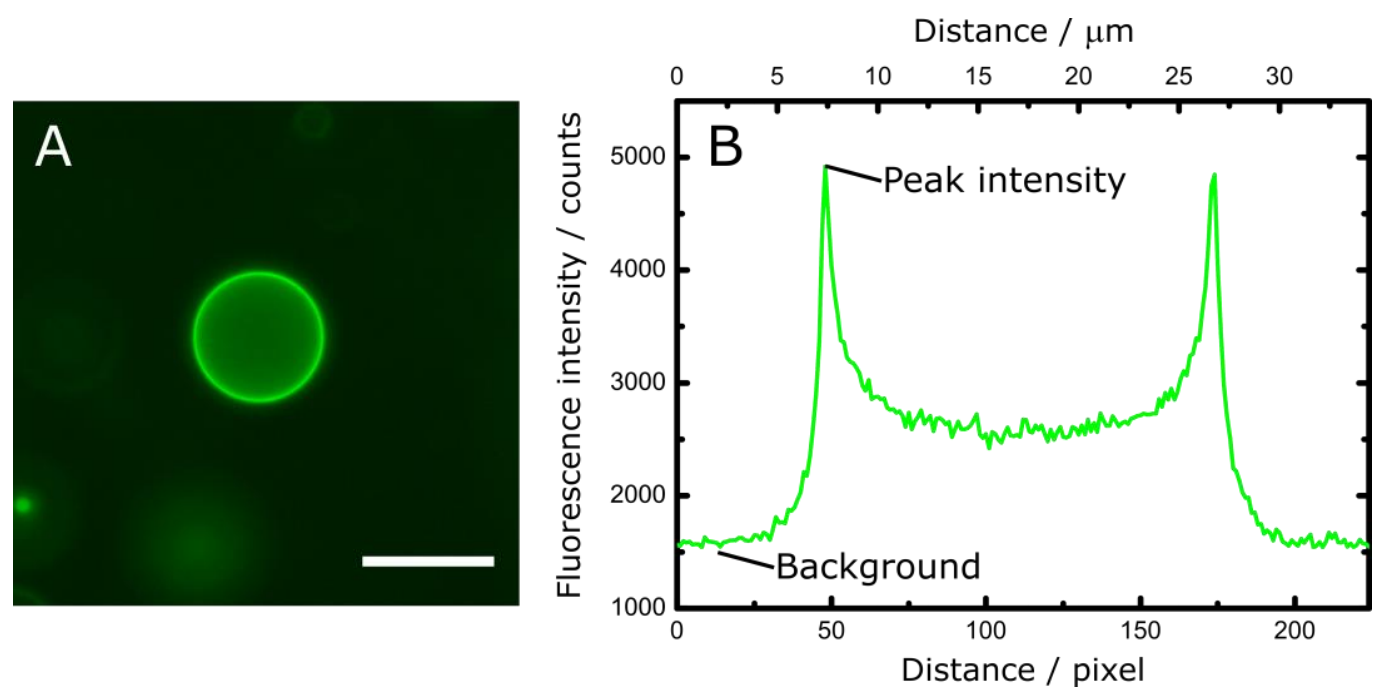

Figure 3.20 (A) Representative epifluorescence micrograph of a $\Delta$ N49-Atto488 GUV. Scale bar $=20 \mu \mathrm{m}$. (B) Representative fluorescence intensity profile with highlighted background and membrane peak fluorescence intensity.

\subsubsection{Confocal laser scanning microscopy}

A confocal laser scanning microscope (CLSM) is a further developed epifluorescence microscope with drastically increased resolution. Fluorophores in the focal plane are excited point by point using a focused laser beam of specific wavelengths which leads to increased $\mathrm{x}$ and y confocality. Through a second pinhole in front of the detector only emitted light from the illuminated focal plane is detected which results in an increased z-confocality. While stepwise 
illumination of fluorophores causes minimal photo damage under normal imaging conditions the excitation energy can be increased in a defined area to systematically bleach fluorophores. Monitoring the fluorescence recovery as a function of time gives information on the movement of molecules inside the imaged membrane. This experiment is called fluorescence recovery after photobleaching (FRAP) and was used in the present work to quantify the 2D diffusion coefficient $D$ of lipids and $\Delta \mathrm{N} 49$-complexes inside GUVs as well as the solid supported part of pore-spanning membranes (s-PSMs).

\section{Fluorescence recovery after photobleaching (FRAP)}

FRAP is a widely used method to investigate the diffusive properties of fluorescently marked lipids and proteins in biological samples. In FRAP the fluorescence recovery is monitored as a function of time after applying a strong bleaching impulse to a certain area of the sample (ROI). While data acquisition for qualitative comparison between identical model systems is straightforward, several factors need to be taken into consideration for the quantitative extraction of diffusion coefficients $D$ from the recovery curve by solving Fick's $2^{\text {nd }}$ law (Equation (3-4)) with $c_{\mathrm{i}}$ being the surface concentration of molecules with different diffusion coefficients $D_{\mathrm{i}}$.

$$
\frac{\partial c_{\mathrm{i}}}{\partial t}=D_{\mathrm{i}} \nabla^{2} c_{\mathrm{i}}
$$

\section{$\underline{\text { GUVS }}$}

In this study diffusion coefficients of molecules inside the membrane of GUVs were determined based on the early work of Axelrod et al. which was further developed by Soumpasis as well as Kang et al. ${ }^{[96-98]}$ The theory requires particle diffusion to be two dimensional and uniform while the region of interest (ROI) in which fluorescence is bleached needs to be circular and the post bleach profile needs to follow a Gaussian intensity profile. To apply this method to the model system of GUVs, the top membrane plane of the GUV (Figure 3.21 A \& B) had to be used to perform FRAP experiments, as this part of the GUV can be seen as a two dimensional planar lipid bilayer. Extracted fluorescence values $F_{\text {raw }}(t)$ had to be corrected for photofading during the measurement $\left(F_{\text {fading }}(t)\right.$, yellow ROI in Figure $3.21 \mathrm{~B}$ ) as well as background corrected $F_{\mathrm{bk}}$, resulting in a corrected intensity $F_{\text {corrected }}(t)$ defined by Equation (3-5). ${ }^{[98]}$

$$
F_{\text {corrected }}(t)=\frac{F_{\text {raw }}(t)-F_{\mathrm{bk}}}{F_{\text {fading }}(t)-F_{\mathrm{bk}}}
$$


Under these conditions a simplified relation between the half time of fluorescence recovery $\left(\tau_{1 / 2}\right)$, which is an intrinsic parameter of the studied model system, and the diffusion coefficient $D$ can be derived. ${ }^{[98]}$ As seen in Equation (3-6) the diffusion coefficient is then dependent on three parameters, $\tau_{1 / 2}$ as well as the nominal bleach radius $r_{n}$ and the effective bleach radius $r_{e}$.

$$
D=\frac{r_{e}^{2}+r_{n}^{2}}{8 \cdot \tau_{1 / 2}}
$$

The nominal bleach radius, usually ranging between 2-3.5 $\mu \mathrm{m}$, is the radius of the ROI set by the user to bleach fluorescence intensity. Standard bleaching of fluorescence while scanning the probe line by line with a laser with uniform circular disc profile would not result in a postbleach profile of Gaussian intensity. Thus, bleaching started in the middle of the ROI and was set to recreate a Gaussian intensity profile. Since bleaching of fluorescence intensity as well as acquisition of the first fluorescence micrograph after bleaching took 0.1 up to $1 \mathrm{~s}$, depending on microscopy settings and used fluorophore, unbleached molecules could diffuse back into the ROI and bleached molecules out of the ROI before the first image after bleaching was recorded. Thus, the effective bleach radius $r_{e}$, which is defined as the radius of the postbleach profile at the height of 0.86 of its depth $K$, was usually larger than $r_{n}$. The effective bleach radius was calculated according to Kang et al. by first extracting the mean postbleach profile by circularly averaging of fluorescence intensity (Figure $3.21 \mathrm{~B}$, red dashed line) using the ImageJ plugin Radial Profile Plot as shown in Figure 3.21 C. Next, $r_{e}$ was calculated by linear interpolation of the data to the $\mathrm{x}$ value of $0.86 \mathrm{~K}$.

To extract $\tau_{1 / 2}, F_{\text {corrected }}$ was then plotted as a function of time and this so called recovery curve fitted with Equation (3-7). ${ }^{[97]}$

$$
F_{\text {corrected }}(t)=A e^{-2 \tau_{1 / 2} / \mathrm{t}}\left[I_{0}\left(2 \tau_{1 / 2} / \mathrm{t}\right)+I_{1}\left(2 \tau_{1 / 2} / \mathrm{t}\right)\right]
$$

Here $I_{0}$ is the modified bessel function of $0^{\text {th }}$ order and $I_{1}$ the modified Bessel function of $1^{\text {st }}$ order. Note that this function has to be fit to the data until the point of full recovery and not until the last data point (see Figure 4.5 B). $D$ was then calculated using Equation (3-6) and the mobile fraction $F_{\mathrm{m}}$ of molecules was calculated using Equation (3-8) with $F_{\infty}$ being the fluorescence intensity at full recovery, $F_{0}$ the intensity directly after bleaching and $F_{\mathrm{i}}$ the intensity before bleaching.

$$
F_{\mathrm{m}}=\frac{F_{\infty}-F_{0}}{F_{i}-F_{0}}
$$



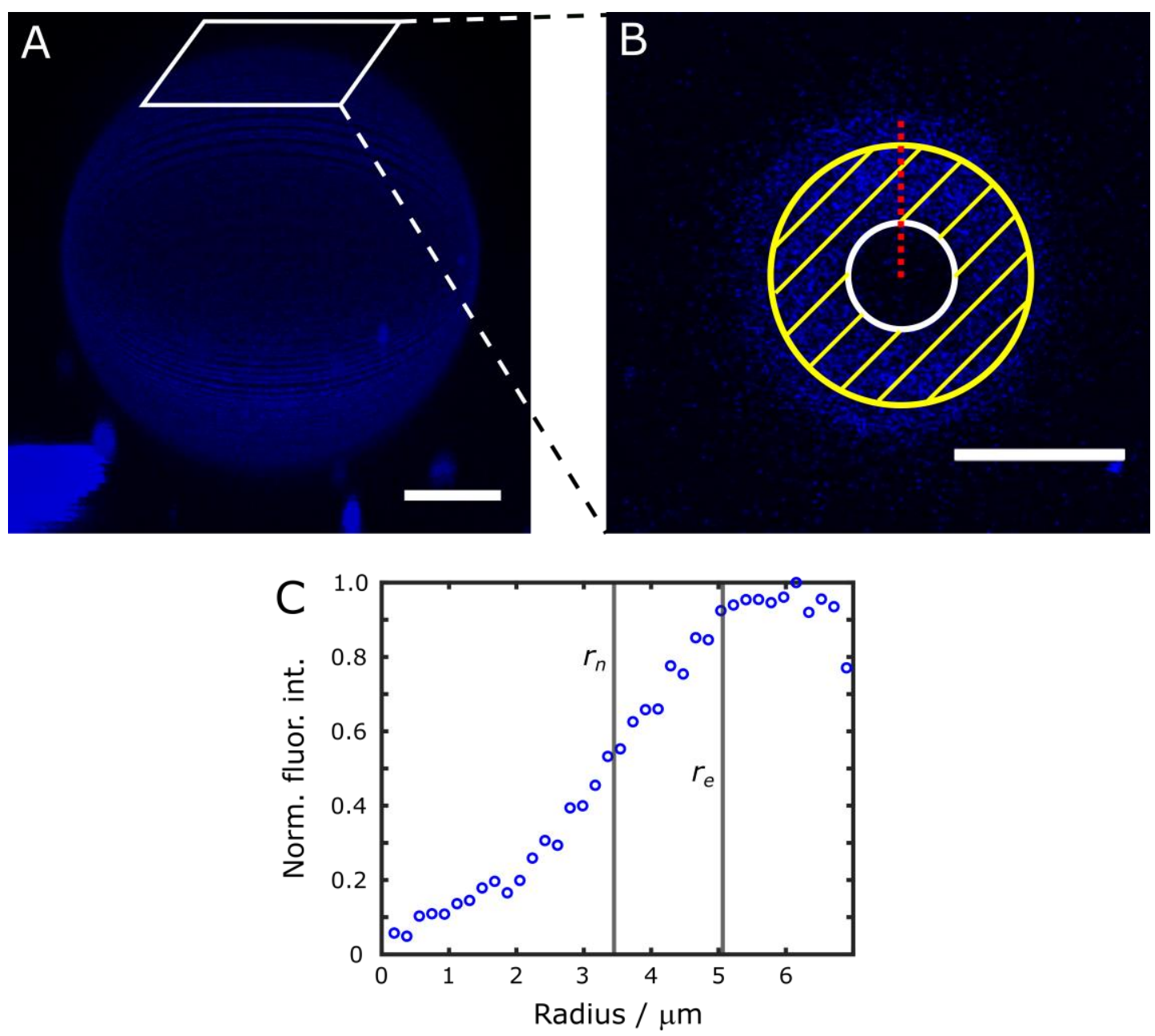

Figure 3.21 (A) 3D reconstruction of z-stacks of an Atto390 DPPE labeled GUV (blue) adhered to the surface containing $\Delta$ N49-Atto488 complex (DOPC/POPE/POPS/cholesterol/Atto390 DPPE; 5/1.95/1/2/0.05 (n/n), nominal $\mathrm{p} / 1=1: 250$ ). The white square highlights the upper most part of the GUV-membrane focused when performing FRAP-experiments. Scale bar $=10 \mu \mathrm{m}$. (B) Fluorescence micrograph of the first frame after bleaching fluorescence intensity (white circle) with a nominal bleach radius of $r_{n}=3.45 \mu \mathrm{m}$ which was also used to read out fluorescence intensity $F_{\text {raw }}(t)$. The yellow donut shaped region was used to extract $F_{\text {fading }}(t)$ while the red dashed line highlights the radius used to extract the normalized integrated radial intensity profile around the center of the bleaching ROI. Scale bar $=10 \mu \mathrm{m}$. (C) Normalized radial intensity profile (blue open circles) with grey vertical lines marking the nominal bleach radius $r_{n}=3.45 \mu \mathrm{m}$ and the extracted effective bleach radius $r_{e}=5.06 \mu \mathrm{m}$.

Experimental procedure

Proteo-GUVs were prepared as described in Chapter 3.2 .4 (DOPC/POPE/POPS/cholesterol/Atto390 DPPE; 5/1.95/1/2/0.05 (n/n), nominal p/1 1:250), filled with 217 mOsM sucrose, and 10-20 $\mu \mathrm{L}$ pipetted into a measuring chamber filled with SNARE buffer A. GUVs adhered and immobilized at the bottom of the petri dish after $\sim 30$ min (Figure 3.21 A). FRAP experiments were performed on the top plane of the GUV for both Atto390 DPPE and $\triangle$ N49-Atto488 with microscopy settings listed in Table 3-11 using a FluoView 1200 CLSM (Olympus, Tokyo, Japan) equipped with a water immersion objective (LUMFLN 60XW / NA 1.1, Olympus, Tokyo, Japan). 
Table 3-11 Microscopy settings to perform FRAP experiments on GUVs.

\begin{tabular}{l|l} 
& User settings \\
\hline laser power 488 & $17 \%(20 \mathrm{~mW})$ \\
laser power 405 & $3 \%$ \\
framerate & $15.4 \mathrm{fps}$ \\
$N$ (frames) & 200 (protein), 100 (lipid) \\
resolution & $256 \times 256$ pixel \\
bleach impulse duration & $48-120 \mathrm{~ms}$ \\
bleach impulse power & $100 \%$ \\
$r_{n}$ & $1.1-3.5 \mu \mathrm{m}$
\end{tabular}

\section{$\underline{\text { PSMs }}$}

Diffusion coefficients of lipids and proteins in the solid supported part of PSMs (s-PSM) were determined using a combination of indirect FRAP experiments and finite element simulations. Due to the gold coating on top of the pore rim of the substrate fluorescence intensity is quenched in this region. ${ }^{[93,99]}$ Thus, neither conventional FRAP experiments nor alternative methods such as fluorescence correlation spectroscopy (FCS) can be used to quantify diffusion coefficients inside the s-PSM. When using indirect FRAP experiments, where fluorescence intensity of one entire f-PSM is quenched (see Figure 3.22 A, white ROI), recovery is mainly dependent on the diffusion of molecules over the pore rim back into the f-PSM. The simplified evaluation method used for the top plane of GUVs can, however, not be followed for s-PSMs due to two reasons. Firstly, recovery into the ROI is not only a function of diffusion of molecules from the s-PSM into the ROI but also a function of diffusion inside the ROI (f-PSM) itself. Since diffusion coefficients are different between the f- and s-PSM, two diffusion coefficients influence the shape of the recovery curve. While in theory possible as shown by Gordon et al., the simplified approach to extract $\tau_{1 / 2}$ from the recovery curve needs a very high signal to noise ratio for such two component systems which was not achieved in this study. ${ }^{[100]}$ Secondly, it is not possible to extract the effective bleach radius from the postbleach profile due to the quenching of fluorescence on the pore rim. Thus, any calculated diffusion coefficient would differ from the 
correct value. ${ }^{[98]}$ As a result, an approach based on comparing recovery curves of indirect FRAP experiments with simulated data, developed by Kuhlmann et al., ${ }^{[61]}$ was used in the present work. ${ }^{[95]}$

FEM simulations (COMSOL Multiphysics v4.4) were performed to solve Fick's $2^{\text {nd }}$ law (Equation (3-4)), with $D_{\mathrm{i}}$ being diffusion coefficients of molecules for the f- and s-PSM. Diffusion coefficients of lipids and proteins in the f-PSM were quantified previously by means of FCS and for simulations fixed to $D_{\text {Lipid }}(\mathrm{f}-\mathrm{PSM})=7.7 \mu \mathrm{m}^{2} \mathrm{~s}^{-1}$ and $D_{\text {Protein }}=3.4 \mu \mathrm{m}^{2} \mathrm{~s}^{-1}$. ${ }^{[85]}$ The PSM was modeled according to the experimental conditions with a porosity of $36 \%$, a pore diameter of $5 \mu \mathrm{m}$ and a sufficiently large surface area with $60 \times 60 \mathrm{~m}^{2}$ (Figure $3.22 \mathrm{~B}$ ). Fluorescence intensity of one f-PSM was then bleached with a Gaussian intensity profile with $r=2.2 \mu \mathrm{m}$ (Figure $3.22 \mathrm{~B}$, red ROI). The surface concentration of the postbleach profile at $t=0 \mathrm{~s}$ was described according to Höök et al. by Equation (3-9) with $c_{\mathrm{eq}}=1, K=2$ and $w=2.2 \mu \mathrm{m} .^{[101]}$

$$
c_{\mathrm{r}, 0}=c_{\mathrm{eq}} \exp \left(-K \exp \left(\frac{-r^{2}}{w^{2}}\right)\right)
$$

Fluorescence recovery curves were then calculated for different diffusion coefficients inside the s-PSM and compared with the experimental data. To account for any fluctuation of fluorescence intensity during the measurement and a comparability of experimental data to simulations, $F_{\text {raw }}(t)$ was normalized and corrected according to Equation (3-5) with yellow ROIs for the extraction of $F_{\text {fading }}(t)$ shown in Figure 3.22 A. $F_{\mathrm{bk}}(t)$ was measured inside the buffer solution above the substrate under identical experimental conditions and microscopy settings.
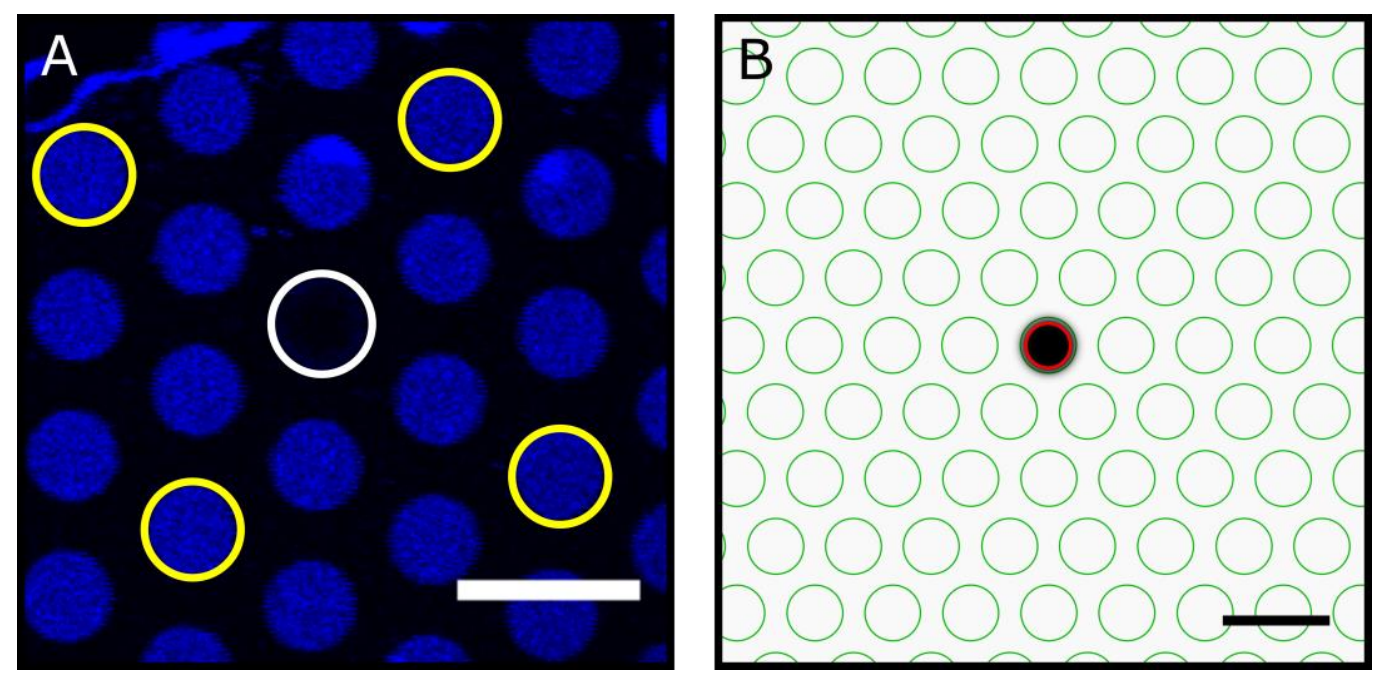

Figure 3.22 (A) Fluorescence micrograph of the first image after bleaching of an indirect FRAP experiment on PSMs with a pore diameter of $5 \mu \mathrm{m}$. The white circle shows the f-PSM in which fluorescence intensity was 
bleached $(r=2.2 \mu \mathrm{m})$ and $F_{\text {raw }}(t)$ extracted from; the yellow circles were used to extract $F_{\text {fading }}(t)$. Scale bar $=10 \mu \mathrm{m}$. (B) Simulated postbleach profile of an indirect FRAP experiment using COMSOL Multiphysics v4.4. The green circles are the f-PSMs with a pore diameter of $5 \mu \mathrm{m}$, the red ROI shows the f-PSM in which fluorescence was bleached with $r=2.2 \mu \mathrm{m}$ and fluorescence recovery was recorded over time. Scale bar $=10 \mu \mathrm{m}$.

Experimental procedure

$\begin{array}{lllll}\text { Proteo-GUVs were prepared as described in Chapter } 3.2 .4 & \end{array}$ (DOPC/POPE/POPS/cholesterol/Atto390 DPPE; 5/1.95/1/2/0.05 (n/n), nominal p/l 1:250) filled with $217 \mathrm{mOsm}$ sucrose and spread on porous substrate with $d_{\text {pore }}=5 \mu \mathrm{m}$ according to Chapter 3.2.5. FRAP experiments were performed by placing a circular bleach ROI (Figure 3.22 A, white ROI, $r=2.2 \mu \mathrm{m}$ ) on top of a f-PSM for both Atto390 DPPE and $\Delta$ N49-Atto488 with microscopy settings listed in Table 3-11 using a FluoView 1200 CLSM (Olympus, Tokyo, Japan) equipped with a water immersion objective (LUMFLN 60Xw, NA 1.1, Olympus, Tokyo, Japan).

Table 3-12 Microscopy settings to perform FRAP experiments on PSMs.

\begin{tabular}{l|l} 
& User settings \\
\hline laser power 488 & $17 \%(20 \mathrm{~mW})$ \\
laser power 405 & $3 \%$ \\
framerate & $15.4 \mathrm{fps}$ \\
$N$ (frames) & 300 (protein), 100 (lipid) \\
resolution & $256 \times 256$ pixel \\
bleach impulse duration & $90 \mathrm{~ms}$ \\
bleach impulse power & $100 \%$ \\
$r_{n}$ & $2.2 \mu \mathrm{m}$
\end{tabular}

\subsubsection{Spinning disc confocal microscopy}

To record fluorescence intensity as a function of time during single-vesicle fusion experiments spinning disc confocal microscopy was used, as it allows for a very high temporal resolution in combination with a high photon sensitivity and low amounts of induced photo toxicity. A schematic illustration of the laser path and important functional components in shown in Figure 
3.23. ${ }^{[102]}$ Fluorophore specific illumination, as in other confocal microscopy setups, is achieved using lasers of specific wavelengths. However, for spinning disc confocal microscopy (SDCM) large portions of the sample are illuminated simultaneously by focusing the laser via a microarray disc containing small lenses through a pinhole disc containing several thousand individual holes (Nipkow disc). The emitted light is reflected by the dichromatic beamsplitter and guided through additional emission filters onto the detector. Due to synchronous rotation of both discs with a rotation speed adapted to the readout frequency of the detector, one image is captured every $30^{\circ}$ of rotation. Overall this setup increases the image acquisition speed compared to CLSMs with the offset of slightly reducing the z-confocality.

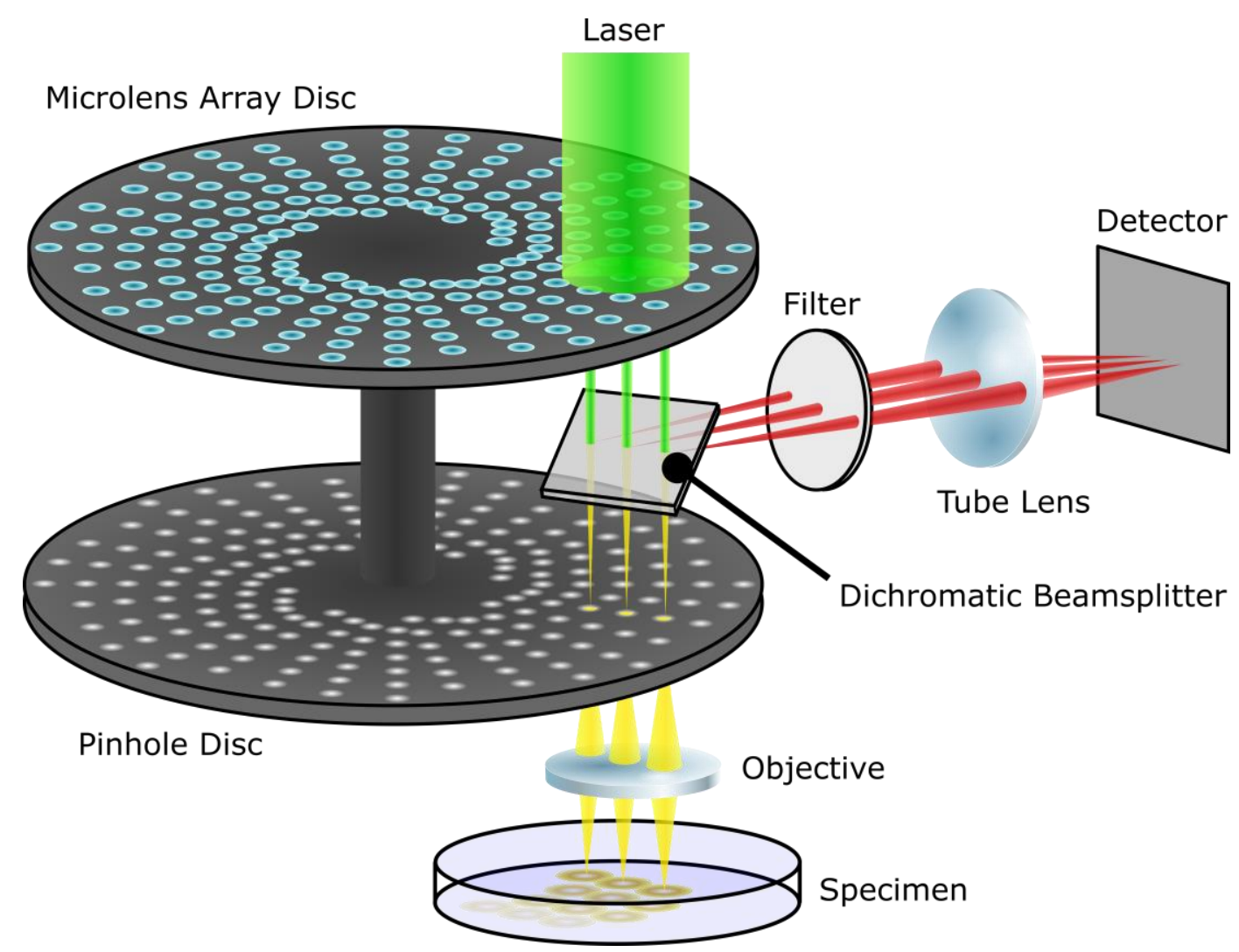

Figure 3.23 Schematic illustration of the laser path of a spinning disc confocal microscope adapted according to Gleisner. ${ }^{[102]}$ Exitation lasers are focused via a microlens array disc through a pinhole disc and the objective on the specimen. Emmitted fluorescence intensity is reflected at a dichromatic beamsplitter and directed through emission filters on the detector surface.

To maximize photon sensitivity and minimize readout time, usually EMCCD or sCMOS cameras serve as detectors. When using only one detector, simultaneous detection of two emitting fluorophores can be achieved by focusing the two wavelength of the emitted image onto different parts of the detector, which makes the time consuming switching between emission filters obsolete. A schematic illustration of the optosplit II (Acal BFi Germany, Dietzenbach, Germany) used in this work is shown in Figure 3.24. While the detected 
observation area is reduced by $50 \%$, both fluorophores are detected $100 \%$ simultaneously with a temporal resolution of up to $10 \mathrm{~ms}$ per frame.

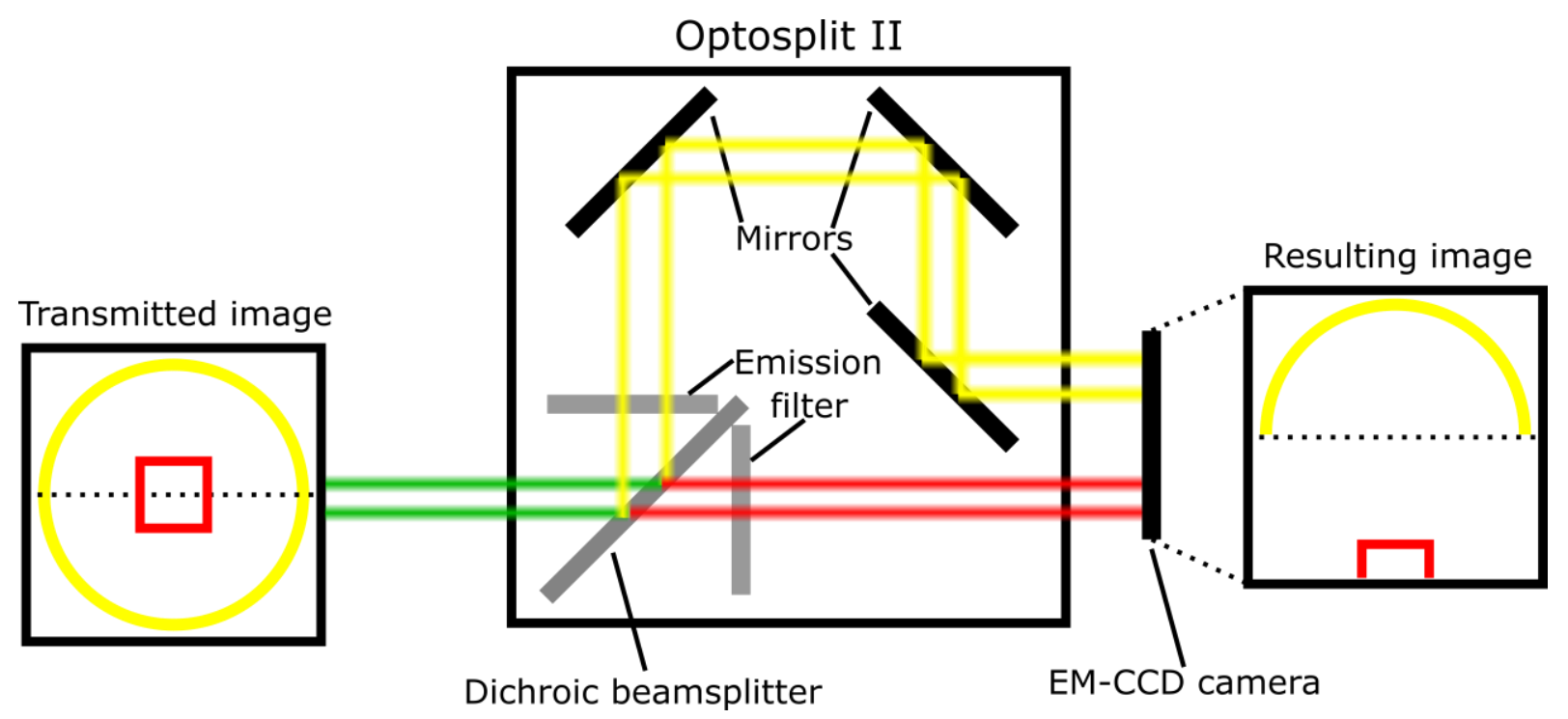

Figure 3.24 Schematic illustration of the interior of the Optosplit II. Combined wavelength of the transmitted image path into the optosplit, are separated by a dichromatic beam splitter, and filtered through emission filters. The transmitted image is split into halves and the separated light of one halve is focused on either side of the detector using the adjustable mirrors. This results in the reduction of observation area by $50 \%$.

\subsubsection{Single-vesicle fusion assay}

To investigate the kinetics and fusion pathways of SNARE-mediated membrane fusion, single-vesicle content release experiments of LUVs fusing with PSMs were recorded by means of SDCM.

\section{Experimental procedure}

For two channel experiments, SRB filled LUVs containing syb 2 and $\triangle N 49$ doped GUVs lipid labeled with $1 \mathrm{~mol} \%$ Atto655 DPPE were prepared according to Chapter 3.2.4 and GUVs were spread on porous substrates with $d_{\text {pore }}=1.2 \mu \mathrm{m}$ according to Chapter 3.2.5. Using an upright spinning disc setup (Yokogawa CSU-X, Rota Yokogawa KG, Wehr, Germany) SRB was excited at $\lambda_{\mathrm{ex}}=561 \mathrm{~nm}$ and Atto655 DPPE at $\lambda_{\mathrm{ex}}=639 \mathrm{~nm}$. The emitted light was focused on either part of the EMCCD camera (iXon 897 Ultra, Andor technology, Belfast, United Kingdom, pixel size $222 \times 222 \mathrm{~nm}^{2}$ ) using an optosplit II. The optosplit was equipped with a H 643 LPXR superflat beamsplitter, a 595/40 ET bandpass, and a 655 LP ET longpass emission filter (AHF Analysetechnik AG, Tübingen, Germany) with properties shown in Figure 3.25. After laser alignment the micro injection unit for LUV addition was focused near the focal point of the objective (LUMFLN 60xW, NA 1.1, Olympus, Hamburg, Germany) and filled with LUV 
solution diluted by a factor of 10 with SNARE buffer B. One PSM was focused and $1 \mu \mathrm{L}$ of LUV solution added directly on top of the PSM. This allowed for uniform vesicle addition between different measurements. Docking to and fusion of LUVs with the PSM was recorded with microscopy settings listed in Table 3-13.

Table 3-13 Microscopy settings to monitor single-vesicle fusion experiments on PSMs in dual color mode.

\begin{tabular}{l|l} 
& User settings \\
\hline laser power 561 & $22 \%$ \\
laser power 639 & $10 \%$ \\
exposure time & $0.02 \mathrm{~s}$ \\
electron multiplier gain & 800 \\
framerate & $48 \mathrm{fps}$ or $20.83 \mathrm{~ms}$ per frame \\
$N$ (frames) & 20000 \\
total time & 6.94 min
\end{tabular}
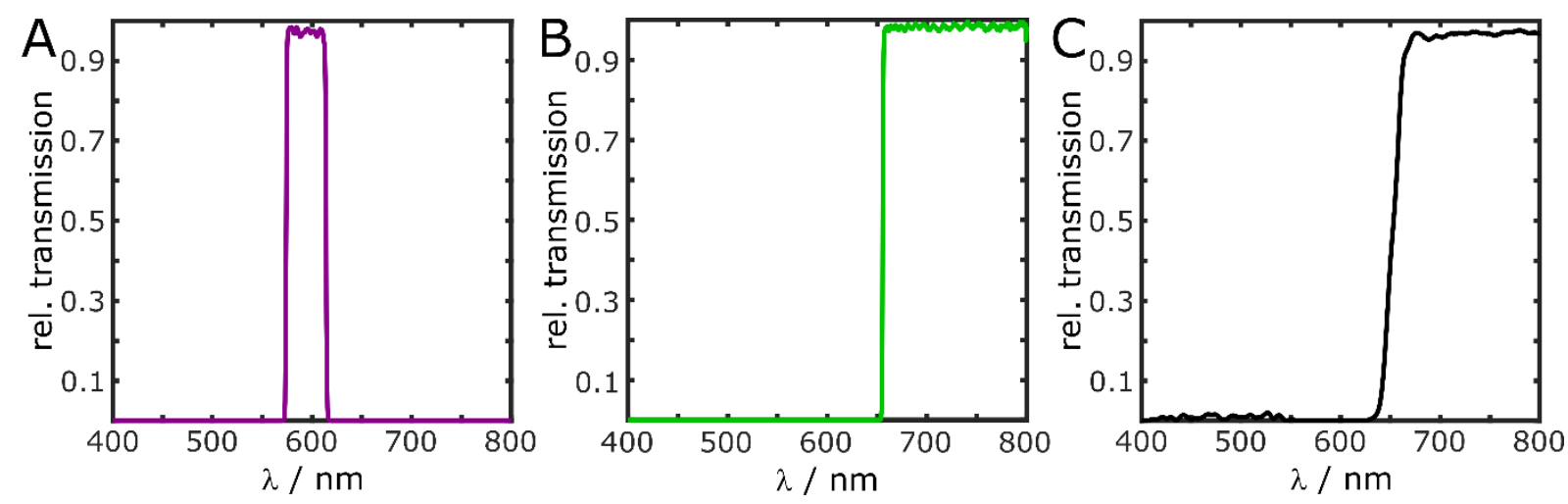

Figure 3.25 Filterset used for two channel single-vesicle fusion experiments. (A) 595/40 ET bandpass filter, (B) 655 LP ET longpass filter and (C) H 643 LPXR superflat beamsplitter.

\section{Data evaluation}

Since SRB fluorescence intensity partially emits into the Atto655 DPPE channel and all single-vesicle fusion experiments were performed under the same conditions, a global factor for crosstalk correction $(C F)$ was determined. Therefore, SRB filled LUVs were immobilized on a functionalized porous substrate and imaged under the same experimental conditions and 
microscopy settings as for single-vesicle experiments. Using ImageJ, the sum of the time series was calculated, vesicles tagged on either side of the split image (left: 1, right: r) and background corrected $\left(\mathrm{BG}_{\mathrm{l}, \mathrm{r}}\right)$ maximum fluorescence intensities $\left(I_{\max }\right)$ extracted. $C F$ was then calculated according to Equation (3-10).

$$
C F=\frac{1}{n} \sum_{i=1}^{n} \frac{\left(I_{\max , \mathrm{l}, i}-B G_{\mathrm{l}}\right)}{\left(I_{\max , \mathrm{r}, i}-B G_{\mathrm{r}}\right)}
$$

Tif-stacks of single-vesicle fusion experiments recorded by means of SDCM were loaded into ImageJ and vesicles that docked mobile to the f-PSM, were tracked using the ImageJ plugin Mosaic. ${ }^{[103]}$ Immobile vesicles docked to the s-PSM were evaluated using a custom made MATLAB-script illustrated in Figure 3.26 which can be downloaded at https://github.com/imey78/FusionAnalysis.git. Each time series was automatically split by the software of the microscope into up to 6 different Tif-stacks, each stack loaded into ImageJ, the sum created, and docked vesicles tagged with a minimum 4 x 4 pixel ROI. ROI-coordinates as well as Tif-stacks were opened with "program 1" in MATLAB. When measuring in the two channel mode, the different halves of the transmitted image were aligned by marking characteristic points such as pore centers on either side of the split image. The ROIs of tagged vesicles in the SRB channel and an additional ROI for dynamic background correction were then transferred to the membrane channel and fluorescence intensity as a function of time read out from the raw data. The saved fluorescence intensity time traces were opened in "program 2" and crosstalk corrected fluorescence intensity of each ROI $n$ plotted as a function of time. Each individual event was then assigned to a specific group using a unique tag, event-tags saved, and the process repeated for all recorded time series $m$. Fluorescence intensity time traces $i$ of the same event-tag were extracted by and opened in "program 3" to set time points of interest e.g. the time of vesicle docking. This data could then be used for further evaluation of e.g. fusion kinetics. 
Program

Image ]

Program 1

Matlab

Program 2

Matlab

\section{Process}

Sum of Tif-stacks

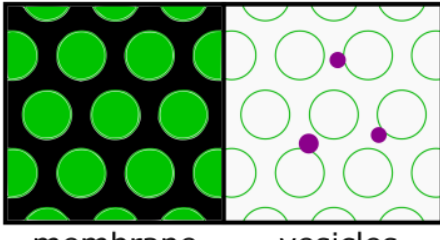

membrane

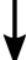

Set vesicle ROIs

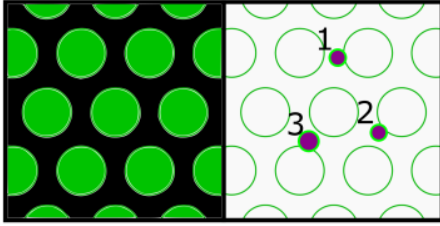

load Tif-stacks \& ImageJ ROIs

$\downarrow$

Align images \& transfer ROIs

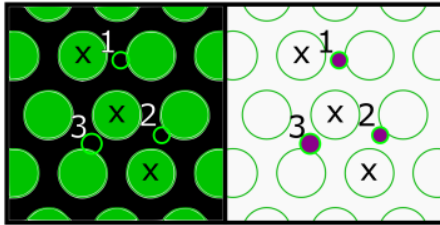

$\downarrow$

Set background ROIs

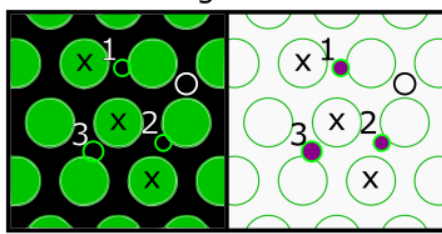

Read out and save fluorescence intensity time traces

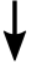

Plot fluor. int. time trace of ROI $n$

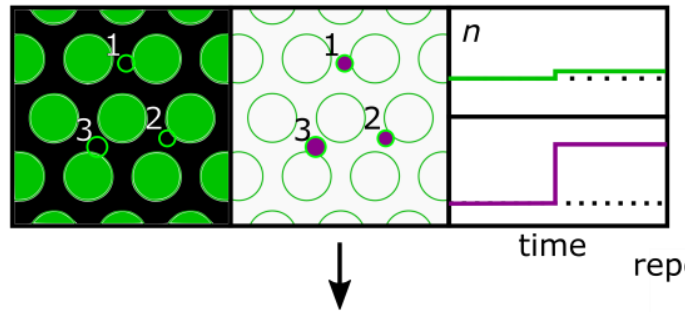

Crosstalk correction \& assign event-tag

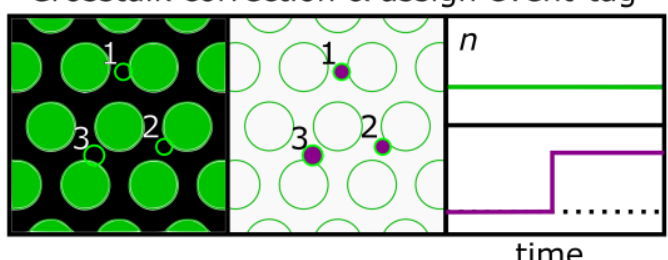

I

save all event-tags

$\downarrow$ repeat for $m$ different

time series 
Program 3

Matlab
Reload all fluor. int. time traces

of 1 event-tag from all $m$ time series
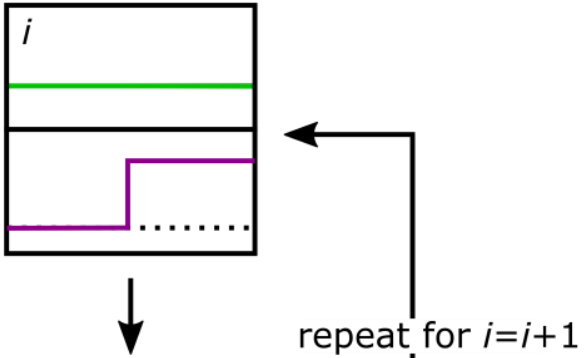

set timepoints in fluor. int.

timetraces of ROI $i$

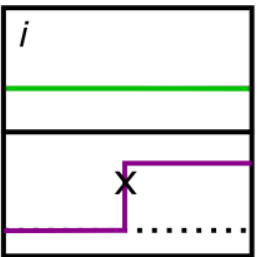

save all timepoints

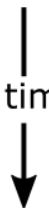

compute

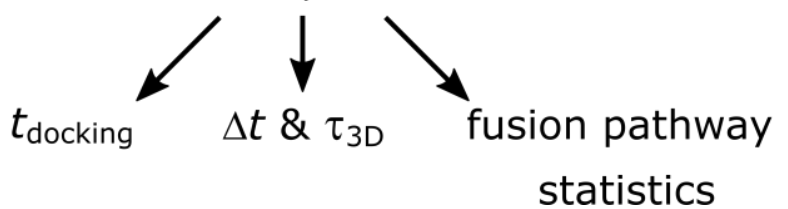

repeat for each

event-tag

Figure 3.26 Process chart illustrating the evaluation of vesicle docking to and fusion with the s-PSM. ImageJ was used to load all Tif-stacks of each time series and manually tag docked vesicles with a ROI. ROI-sets and Tif-stacks were opened with program 1, membrane channels aligned and background ROIs selected. Fluorescence intensity time traces were saved, opened in program 2, and the crosstalk corrected. Specific event-tags were assigned to each docked vesicle $n$, data saved, and all steps repeated for all recorded time series $m$. Program 3 scanned the complete data set for identical event-tags and loaded the respective fluorescence intensity time traces. Important time points were set and the data saved for further evaluation. 


\section{Results}

-Parts of this work have been published in Biophysical Journal ${ }^{[95]}$

Single-vesicle fusion assays mimicking the process of membrane fusion prior to neurotransmitter release in synapses have been developed over the past decades. ${ }^{[10,48,65,75,77,104]}$ These in vitro assays helped to better understand the molecular mechanisms and kinetics of SNARE-mediated membrane fusion and usually monitored fusion of highly curved vesicles with a planar supported lipid bilayer (SLB) on a single-vesicle level by means of fluorescence microscopy. ${ }^{[48,75,77]}$ Vesicles were filled with water soluble dyes to directly monitor fusion pore formation, however, SLBs lack of a second aqueous compartment for the content to be released in. ${ }^{[36,74,82,105]}$ A model membrane system that is both planar, thus easily accessible by fluorescence microscopy, and provides enough space on both sides of the membrane are pore-spanning membranes (PSMs). Based on the work of Ines Höfer, Lando Schwenen recently developed a lipid mixing based single-vesicle fusion assay using PSMs that led to valuable insights in the process of SNARE-mediated membrane fusion. ${ }^{[84,85]}$

In the present work this model system was extended to directly visualize fusion pore formation by means of content release while simultaneously lipid mixing was monitored to gather detailed information about different fusion pathways. To achieve this, the v-SNARE synaptobrevin 2 was reconstituted into large unilamellar vesicles (LUVs) filled with the water-soluble fluorophore sulforhodamine $B(S R B)$ and the t-SNARE acceptor complex $\Delta$ N49 reconstituted into PSMs that were lipid labeled with Atto655 DPPE (Figure 4.1 A). Single-vesicle docking to and fusion with the PSM was then recorded by means of high speed dual color spinning disc confocal microscopy (Figure 4.1 B).

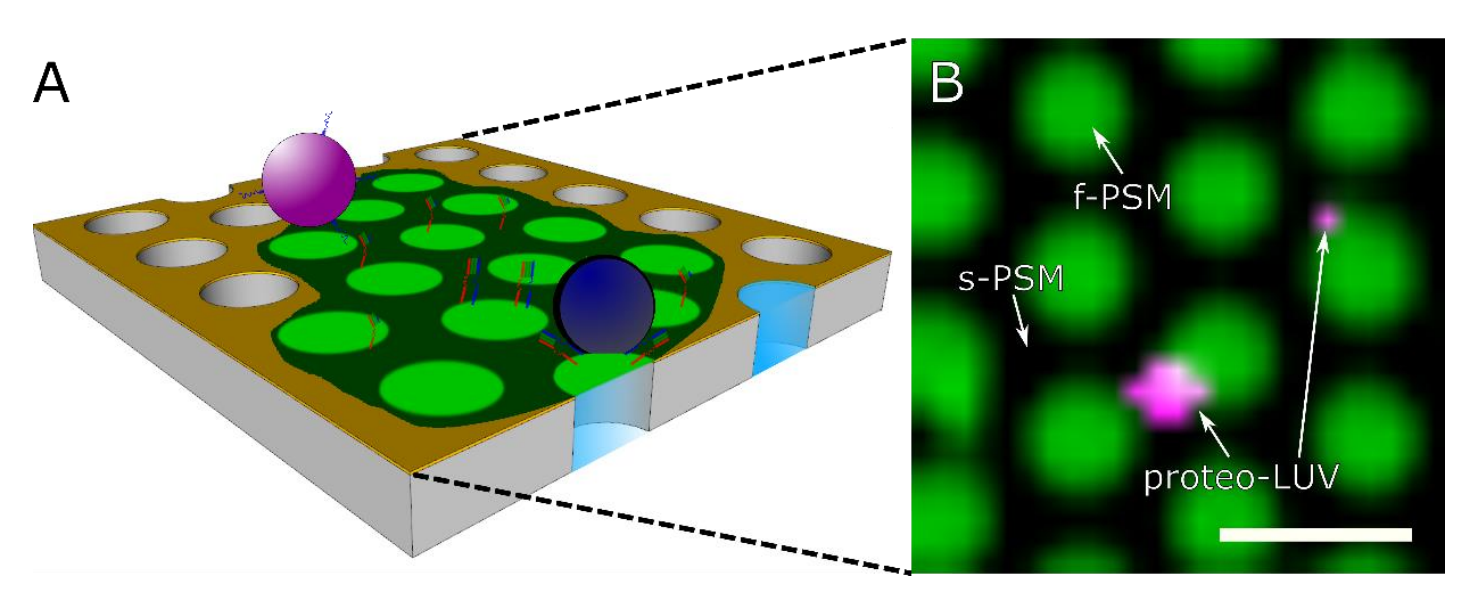

Figure 4.1 (A) Schematic illustration of the single-vesicle content release assay based on PSMs and SRB filled LUVs. (B) Fluorescence micrograph of Atto655 DPPE labeled PSMs (false colored in green) with reconstituted $\triangle \mathrm{N} 49$-complex (DOPC/POPE/POPS/cholesterol/Atto655 DPPE; 5/1.9/1/2/0.1 (n/n), nominal p/l 1:500). 
Fluorescence intensity is quenched due to the underlying gold surface inside the solid supported part of the PSM (s-PSM). Proteo-LUVs (false colored in magenta) containing syb 2 and filled with SRB (43 mM, same lipid composition and $\mathrm{p} / \mathrm{l}$ ratio) are docked to the s-PSM. Scale bar: $2 \mu \mathrm{m}$.

\subsection{Functional reconstitution of SNAREs into liposomes}

Functional reconstitution of the minimal fusion machinery into lipid bilayers and the incorporation of SRB into LUVs, without influencing their membrane stability, was essential for single-vesicle fusion experiments. SRB filled LUVs containing syb 2 (DOPC/POPE/POPS/cholesterol; 5/2/1/2 ( $n / n)$, nominal p/1 1:500, 43 mM SRB) were prepared as described in Chapter 3.2.4 with the reconstitution protocol based on the work of Pobbati et $a l .{ }^{[51]}$ Successful content incorporation was tested by means of UV/Vis-spectroscopy. At high concentrations aromatic parts of SRB (see Figure 3.3) that are responsible for the fluorescence stack on top of each other which leads to a dimerization of the molecules. ${ }^{[106]}$ The induced change in the electronic structure of SRB leads to concentration quenching of fluorescence which is also reflected in a change in the absorption behavior of the dye as seen in Figure 4.2 A. At high concentrations (Figure 4.2 A, I) SRB exhibits two distinct peaks in the absorbance spectra at $565 \mathrm{~nm}$ and $528 \mathrm{~nm}$ of which only the absorbance of light at $565 \mathrm{~nm}$ leads to the emission of fluorescence. Lysis of SRB-LUVs after addition of Triton X-100 leads to an increase in absorbance at $565 \mathrm{~nm}$ while absorbance at $528 \mathrm{~nm}$ remains constant. This increase in absorbance at higher wave numbers is directly coupled with increased fluorescence intensity of SRB due to monomerization and thus dequenching of the dye. This increase of fluorescence due to dilution was further used to test for functional reconstitution of syb 2 and $\Delta \mathrm{N} 49$ into LUVs and SUVs in a bulk content release assay described in Chapter 3.2.4. Briefly, the two vesicle populations are mixed, SRB fluorescence excited at $\lambda_{\mathrm{ex}}=560 \mathrm{~nm}$, and fusion induced, time dependent fluorescence increase detected at $\lambda_{\mathrm{em}}=590 \mathrm{~nm}$ (Figure $4.2 \mathrm{~B} \mathrm{I}$ ). Vesicle lysis was induced at the end of the time series as an internal standard to extract a mean fusion efficiency of $30 \%$ and a half-life time of $t_{1 / 2}=641 \mathrm{~s}$. SNARE specificity of fluorescence dequenching was tested by blocking the acceptor complex with the soluble part of syb 2 (syb 2 aa 1-96) prior to protein reconstitution which is known to prevent docking of syb 2 containing vesicles and thus inhibits fusion. ${ }^{[51]}$ No increase in fluorescence as a function of time was recorded for this control measurement (Figure 4.2 B II). This does not only show the SNARE specificity of this process but also the long term stability of content labeled LUVs. The mean diameter of LUVs produced by extrusion through a $400 \mathrm{~nm}$ membrane was determined previously to be $240 \pm 100 \mathrm{~nm} .^{[86]}$ 

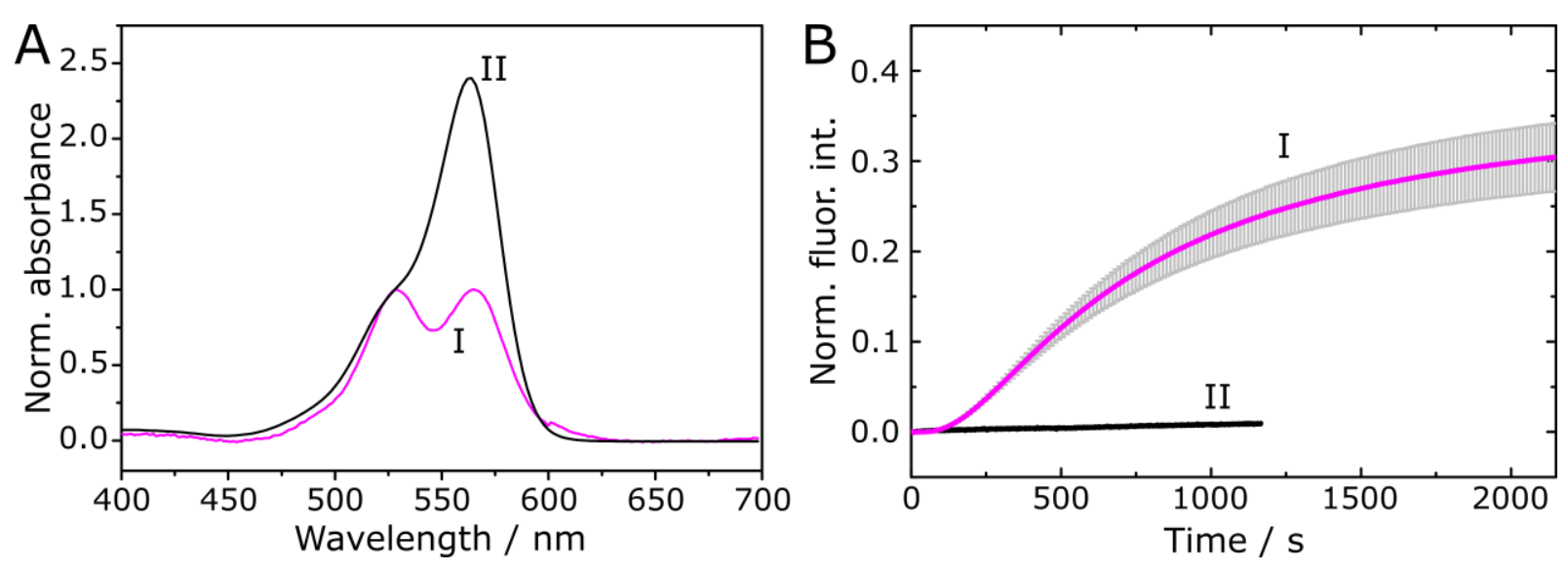

Figure 4.2 (A) Normalized UV/Vis absorbance spectra of encapsulated, concentration quenched (I) and free, dequenched (II) SRB. (B) Time resolved fluorescence intensity of a bulk content release assay of syb 2 doped, SRB filled LUVs fusing with $\triangle N 49$ containing SUVs to test for functional reconstitution of proteins into liposomes (I). Control experiment with blocked $\Delta \mathrm{N} 49$ by displacing Sb49-96 with Sb 1-96 prior to protein reconstitution (II).

\subsection{Reconstitution efficiency of $\Delta$ N49-complex into GUVs}

- Epifluorescence measurements were carried out by Loan Vuong as part of her bachelor thesis -

Besides pure functionality it is very important to know the total amount of SNAREs present in the respective model membrane as this might influence the docking behavior and fusion kinetics on a single-vesicle level. Starting from a nominal protein to lipid ratio of 1:500 (DOPC/POPE/POPS/cholesterol; 5/2/1/2 $(n / n)$ ) the reconstitution efficiency $R$ of syb 2 and $\Delta$ N49 into SUVs was previously quantified by Jan Kuhlmann via a nycodenz flotation assay. ${ }^{[61]}$ While an overall loss of lipid and protein material was monitored, probably resulting from the different size exclusion steps, proteins were reconstituted with efficiencies $>90 \%$. For the final step of the preparation of syb 2 containing LUVs the effective p/l ratio was estimated to be 1:600. By applying Equation (4-1) with a mean LUV radius of $r=120 \mathrm{~nm}$, a protein to lipid ratio of $\mathrm{p} / \mathrm{l}=1 / 600$, and a lipid area of $A_{1}=0.65 \mathrm{~nm}^{2}$ this leads to a total number of $\sim 930 \mathrm{syb} 2$ molecules per LUV or $\sim 5000$ proteins per $\mu \mathrm{m}^{2} .{ }^{[107]}$ Assuming no preferred orientation of syb 2 during reconstitution, this leaves 460 syb 2 molecules facing with their SNARE binding motifs into the outer solution or a density of $\sim 2500$ molecules per $\mu \mathrm{m}^{2}$. However, the reconstitution efficiency of $\Delta \mathrm{N} 49$-complex into GUVs could not be quantified in this study.

$$
N_{\text {protein }}=\frac{4 \pi r^{2}}{A_{1}} \cdot 2 \cdot \mathrm{p} / \mathrm{l}
$$


In the present work successful reconstitution of the t-SNARE acceptor complex $\Delta \mathrm{N} 49$ was visualized and quantified by means of fluorescence microscopy. As described in Chapter 3.2.3 the acceptor complex was assembled from syx 1A, SNAP25a and a Sb49-96 fragment labeled with Atto488. Labeling at the small fragment ensured to only visualize the fusion active 1:1 complex while labeling of either syx 1A or SNAP25a would also visualize remaining fusion inactive 2:1 complex. Fusion activity of the labeled acceptor complex inside SUVs was proven in a bulk content release assay (Figure $4.3 \mathrm{C}$ ) as described in Chapter 3.2.4. GUVs were then prepared as described in Chapter 3.2.4 while additionally labeling the GUV membrane with $0.5 \mathrm{~mol} \%$ Atto390 DPPE. As seen in Figure 4.3 A the lipid dye is homogeneously distributed between different GUVs and inside the GUV membrane. Figure 4.3 B shows successful reconstitution of $\Delta \mathrm{N} 49-$ Atto 488 into the GUV membrane. However, in some cases the protein formed clusters inside the bilayer and the total amount of protein differed between GUVs.

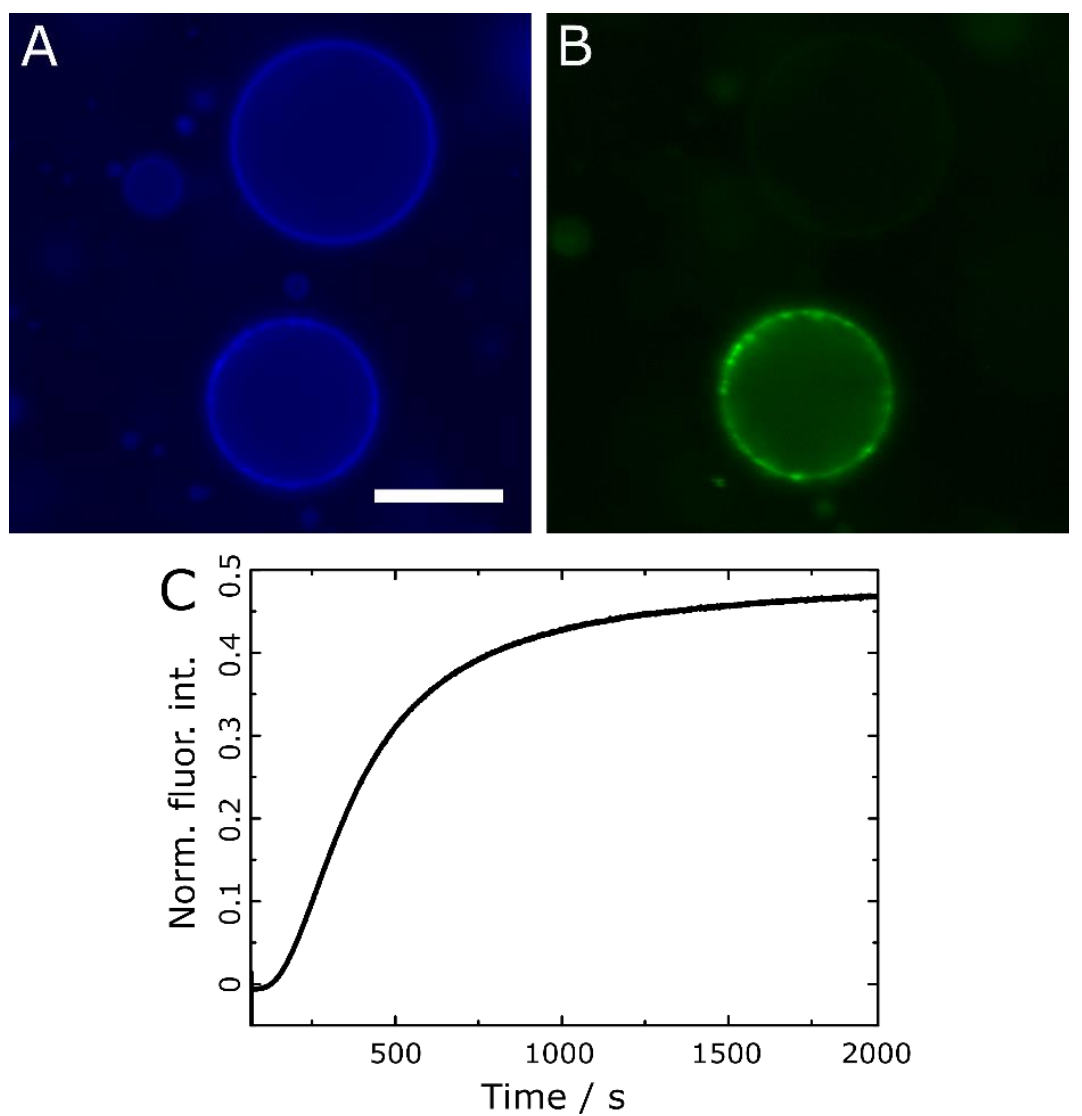

Figure 4.3 Fluorescence micrographs of (A) the Atto390 DPPE labeled GUV membrane and (B) $\Delta$ N49-Atto488 fluorescence intensity (DOPC/POPE/POPS/cholesterol/Atto390 DPPE; 5/1.95/1/2/0.05 (n/n), nominal p/1 1:500). Scale bar $=20 \mu \mathrm{m}$. (C) Fluorescence intensity over time of a bulk content release assay of syb 2 containing LUVs fusing with $\Delta$ N49-Atto488 doped SUVs to test the fusogenity of the labeled acceptor complex. 
To quantify this observation, the reconstitution efficiency $R$ was determined as described in Chapter 3.3.1 with slight modifications based on the work of Aimon et al. ${ }^{[94,95]}$ Briefly, membrane peak fluorescence intensities of GUVs imaged in the equatorial plane via epifluorescence microscopy of known Atto488-DPPE concentrations were compared with $\Delta \mathrm{N} 49$-Atto488 fluorescence intensities in GUVs acquired using identical microscopy settings. The settings were optimized to result in large differences in detector counts between fluorophore concentrations while staying in the range where the number of emitted photons is linear to detector counts of the used EMCCD camera. Peak membrane intensities of known fluorophore concentrations were used to extract a calibration factor by fitting a linear weighted fit to the data (Figure $4.4 \mathrm{~A}$, red line). The slope of the weighted fit $M_{\mathrm{ref}}=47300 \mathrm{counts} / \mathrm{mol} \%$ was then used together with the degree of labeling of the protein $(D O L=35 \%)$ to calculate reconstitution efficiencies of $\Delta \mathrm{N} 49$ into $N=1015$ individual GUVs using Equation (3-3). Fitting a log-normal distribution to the data resulted in a median reconstitution efficiency of $R=26 \pm 24 \%$ or an effective $\mathrm{p} / \mathrm{l}$ ratio of $1: 1900$. Simultaneously, the mean proteo-GUV diameter was determined to be $d_{\mathrm{GUV}}=15 \mu \mathrm{m}$. With a lipid area of $0.65 \mathrm{~nm}^{2}$ and using Equation (4-1) this would lead to $\sim 800$ proteins $/ \mu \mathrm{m}^{2}$ membrane facing towards the inner solution of the GUV. ${ }^{[107]}$
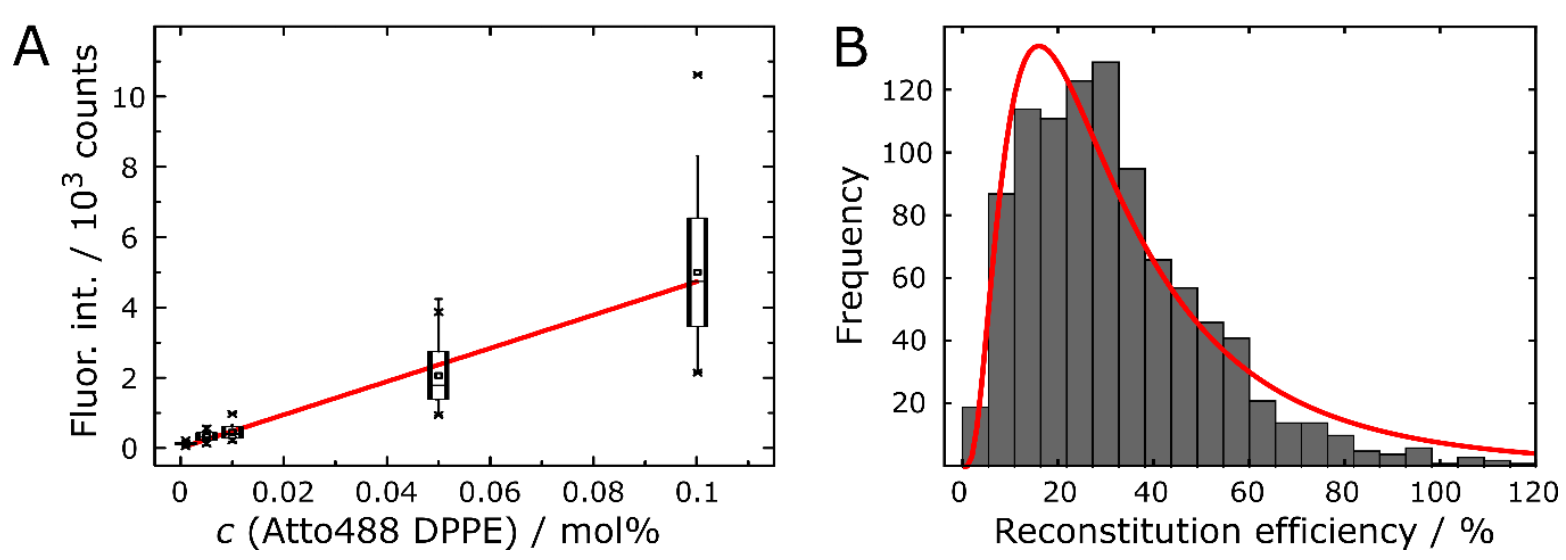

Figure 4.4 Determination of the reconstitution efficiency $R$ of $\Delta$ N49-Atto488 into GUVs. (A) Concentration of Atto488 DPPE as a function of detector counts of 5 different dye concentrations $(c / \mathrm{mol} \%=0.001,0.005,0.01$, $0.05,0.1 ; N=98,218,336,196,98)$. Fitting a weighted linear regression to the data (red line) results in the extraction of calibration factor $M_{\text {ref }}=47300$ counts $/ \mathrm{mol} \%$. (B) Reconstitution efficiency of $\Delta \mathrm{N} 49$ in GUVs starting with a nominal p/l ratio of 1:500 $(N=1015)$. Values of $R>120 \%$ are not displayed in the plot $(N>120 \%=49)$. Fitting a log-normal distribution to the data results in a median reconstitution efficiency of $26 \pm 24 \%$ and an effective p/l ratio of 1:1900. 


\subsection{Mobility of proteins and lipids in model membrane systems}

- FRAP experiments were carried out by Kira Herwig as part of her bachelor thesis -

\subsubsection{Mobility of lipids and $\Delta$ N49-complexes in GUVs}

The mobility of lipids and proteins is important for the process of SNARE-mediated membrane fusion in vivo and therefore it is necessary to investigate the mobility of components in the respective model membrane systems. ${ }^{[108]}$ As GUVs are the starting material to form pore-spanning membranes (PSMs) and known to enable free 2D diffusion of molecules inside their membrane, they are used as a reference system in this work. Thus, the diffusion coefficient of the t-SNARE acceptor complex $\triangle \mathrm{N} 49$ was first quantified in free GUVs by means of fluorescence recovery after photobleaching (FRAP) experiments. Proteo-GUVs (DOPC/POPE/POPS/cholesterol/Atto390 DPPE; 5/1.95/1/2/0.05 ( $n / n)$, nominal p/1 1:250) were prepared and FRAP experiments performed as described in Chapter 3.2.4 and Chapter 3.3.2, respectively. Briefly, fluorescence was bleached in a circular ROI in the top plane of the GUV (Figure 4.5 A, white ROI, $r_{\mathrm{n}}=3.17 \mu \mathrm{m}$ ) and fluorescence recovery recorded as a function of time. The data was corrected for focus drift/photofading during ilumination and background fluorescence intensity. The specific diffusion time $\tau_{1 / 2}$ was extracted by fitting Equation (3-7) to the normalized, corrected recovery curve (Figure 4.5 B, red line). ${ }^{[97]}$ To compensate for diffusing molecules into the ROI during bleaching, the effective bleach radius $r_{e}$ was extracted from the postbleach profile (Figure 3.21 B, C). The diffusion coefficient $D$ was then calculated using Equation (3-6) and the mobile fraction $F_{\mathrm{m}}$ of molecules using Equation (3-8). ${ }^{[98]}$ 


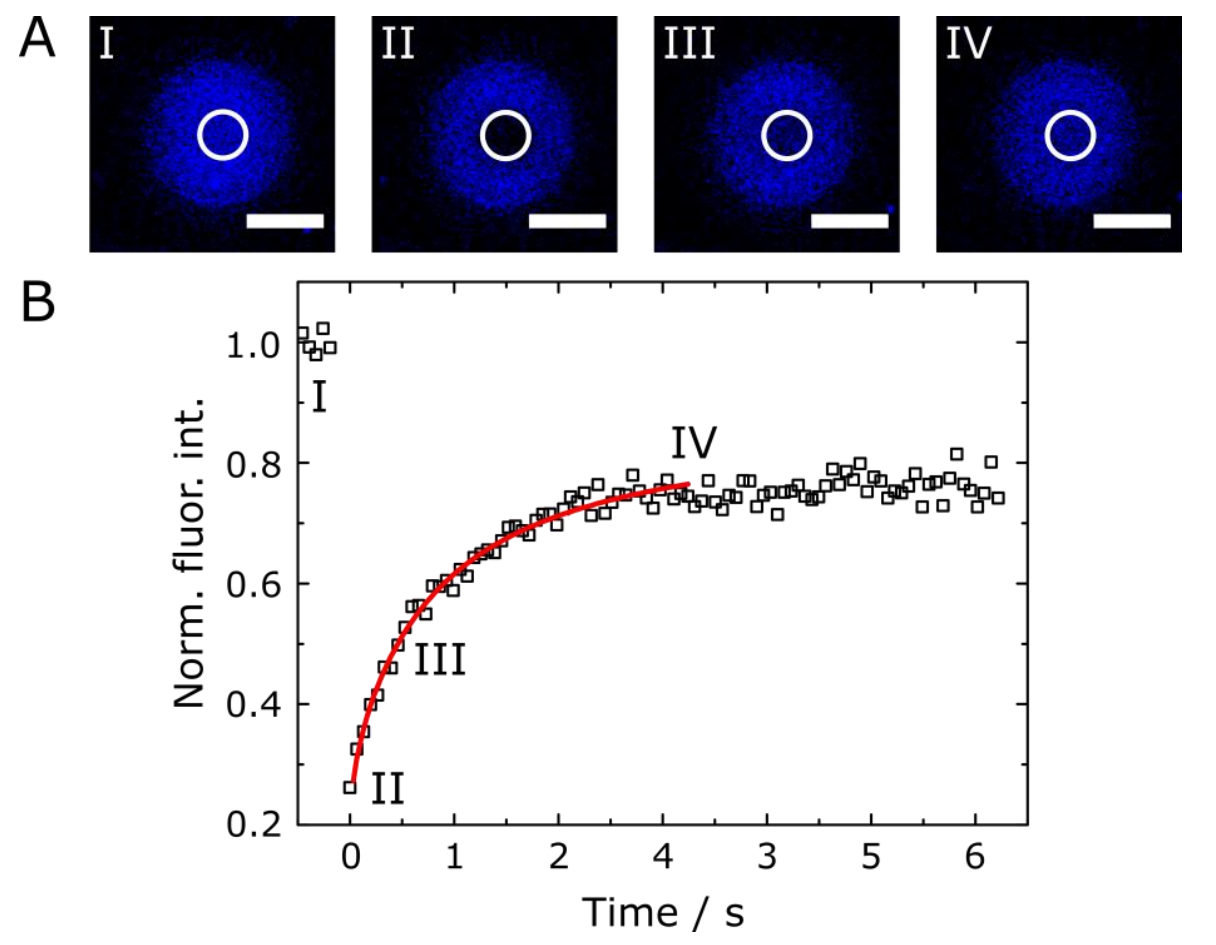

Figure 4.5 (A) Fluorescence micrographs of a FRAP experiment on the top plane of a GUV (DOPC/POPE/POPS/cholesterol/Atto390 DPPE; 5/1.95/1/2/0.05 (n/n), nominal p/l 1:250). After acquisition of pre bleach fluorescence (I) lipid dye molecules were bleached (white circle, $r_{\mathrm{n}}=3.17 \mu \mathrm{m}, r_{\mathrm{e}}=6.67 \mu \mathrm{m}$ ) and fluorescence recovery was monitored as a function of time. Scale bar $=10 \mu \mathrm{m}$. (B) Normalized fluorescence recovery as a function of time was read out from the white ROI in A. The red line is the fit of Equation (3-7) to the data leading to the diffusion time $\tau_{1 / 2}=0.52 \mathrm{~s}$.

Both Atto390 DPPE and $\triangle \mathrm{N} 49$ were fully mobile inside the GUV membrane with $F_{\mathrm{m}}($ Atto488 DPPE $)=100 \pm 8 \%$ and $F_{\mathrm{m}}(\Delta \mathrm{N} 49-$ Atto 488$)=100 \pm 7 \%$. Fitting a normal distribution function to the histogram of diffusion coefficients (Figure 4.6 A, B, red line) results in diffusion coefficients of $D_{\text {Atto390 DPPE }}=9 \pm 3 \mu \mathrm{m}^{2} \mathrm{~s}^{-1}(N=25)$ and $D_{\triangle \mathrm{N} 49 \text {-Atto488 }}=5 \pm 3 \mu \mathrm{m}^{2} \mathrm{~s}^{-1}$ $(N=26)$ with standard deviations as errors. 

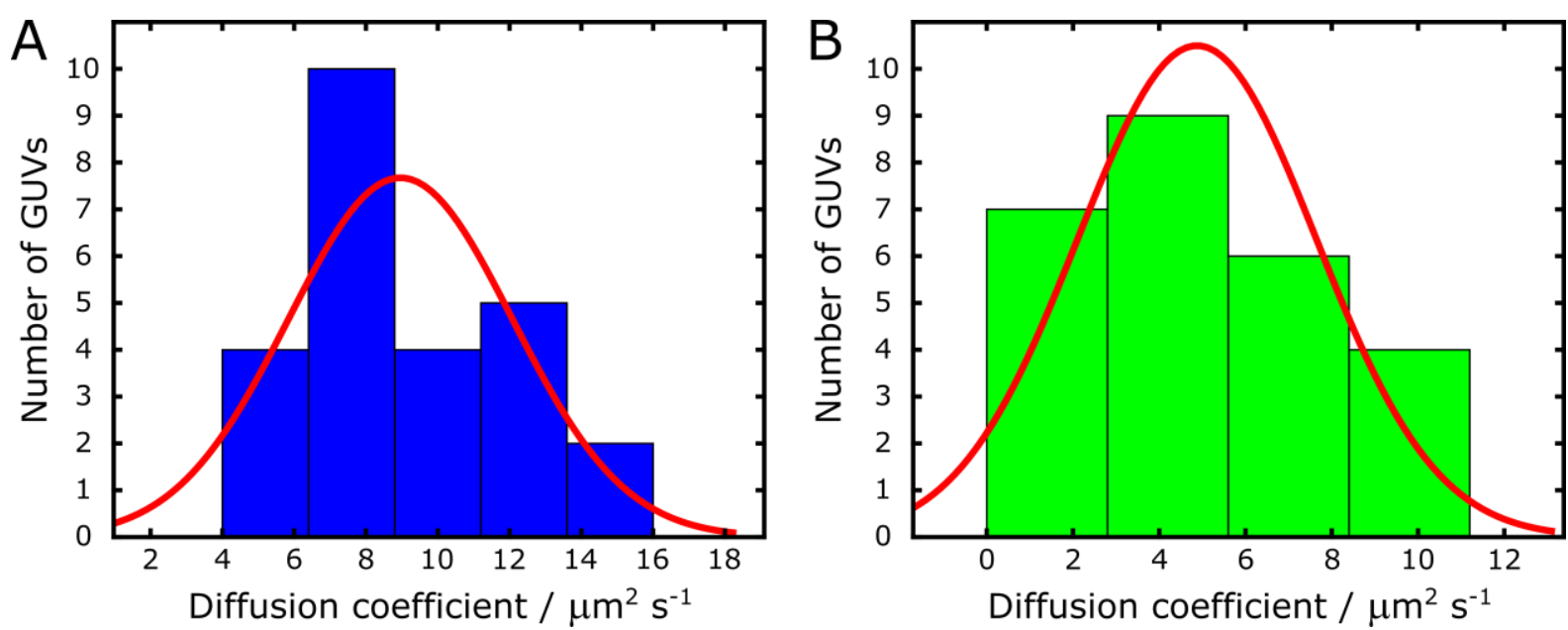

Figure 4.6 Diffusion coefficients of lipids and $\Delta$ N49-Atto488 inside GUVs. The red lines are the result of fitting a normal distribution to the data with median values for the diffusion coefficient of lipids $D_{\text {Atto390 DPPE }}=9 \pm 3 \mu \mathrm{m}^{2} \mathrm{~s}^{-1}(N=25)(\mathrm{A})$ and proteins $D_{\triangle \mathrm{N} 49-\mathrm{Att} 0488}=5 \pm 3 \mu \mathrm{m}^{2} \mathrm{~s}^{-1}(N=26)$.

\subsubsection{Mobility of lipids and $\Delta$ N49-complexes in PSMs}

Lipid and t-SNARE mobility in pore-spanning membranes (PSMs) is essential for the fusion of v-SNARE doped vesicles with the target membrane. PSMs were prepared as described in Chapter 3.2.5 by spreading GUVs with reconstituted $\Delta$ N49-Atto488 complex (DOPC/POPE/POPS/cholesterol/Atto390 DPPE; 5/1.95/1/2/0.05 (n/n), nominal p/1 1:250) on porous substrates with pore diameters of $5 \mu \mathrm{m}$. PSMs consist of two distinct membrane parts, a freestanding part spanning the holes of the substrate (f-PSM) and a solid supported part covering the gold/mercapto-hexanol functionalized pore rim (s-PSM). As molecules exhibit different diffusive behavior in the two different parts of the PSM they have to be investigated separately. Previously, Schwenen et al. performed fluorescence correlation spectroscopy (FCS) experiment inside the f-PSM using a labeled transmembrane domain (TMD) of syx 1A and labeled syx 1A (aa 183-288) as well as lipid markers. ${ }^{[85]}$ The obtained diffusion coefficients were $\quad D_{\text {syx 1A-TMD }}(\mathrm{f}-\mathrm{PSM})=3.4 \pm 0.2 \mu \mathrm{m}^{2} \mathrm{~s}^{-1}, \quad D_{\text {syx } 1 \mathrm{~A}}(\mathrm{f}-\mathrm{PSM})=2.3 \pm 0.5 \mu \mathrm{m}^{2} \mathrm{~s}^{-1}, \quad$ and $D_{\text {DPPE }}(\mathrm{f}-\mathrm{PSM})=7.7 \pm 0.4 \mu \mathrm{m}^{2} \mathrm{~s}^{-1}$. Due to the quenched fluorescence inside the s-PSM, neither FCS experiments nor FRAP experiments as described for GUVs could be performed in this part of the PSM. However, by bleaching the fluorescence of one individual f-PSM the recovery of f-PSM fluorescence is dominated by the diffusion of molecules over the s-PSM surrounding the pore. These so called indirect FRAP experiments, as previously reported by Kuhlmann et $a l$. for the syx-TMD, can be used to describe the diffusion of molecules inside the s-PSM. ${ }^{[61]}$ Conventional data analysis where the diffusion coefficient is directly derived from the half-life time of fluorescence recovery, as used in Chapter 4.3.1, is not applicable for indirect FRAP 
experiments due to two reasons. First, the effective bleach radius could not be extracted from the postbleach profile since the Gaussian intensity profile is cut off at the edge of the pore, due to the lack of fluorescence intensity on the pore rim. Secondly, the recovery of fluorescence inside the bleach ROI is influenced by the diffusion of molecules over the pore rim but also inside the f-PSM. Such a two component system would need a higher signal to noise ratio of the data than achieved in this work to lead to accurate values of $\tau_{1 / 2}$ extracted from the recovery curve. ${ }^{[100]}$ Thus a method was used which is based on the comparison of recovery curves with simulated FRAP experiments. ${ }^{[61]}$ Briefly, FRAP experiments were performed as described in Chapter 3.3.2 with exemplarily fluorescence micrographs shown in Figure 4.7 A. Finite element simulations were performed as described in Chapter 3.3.2 to model the 2D diffusion of lipids and proteins inside the PSM after bleaching of fluorescence of a f-PSM (Figure 4.7 B). Values for the diffusion inside the f-PSM were fixed to $D_{\text {Lipid }}$ (f-PSM) $=7.7 \mu \mathrm{m}^{2} \mathrm{~s}^{-1}$ and $D_{\text {Protein }}(\mathrm{f}-\mathrm{PSM})=3.4 \mu \mathrm{m}^{2} \mathrm{~s}^{-1}$ (values derived from FCS measurements) and fluorescence recovery inside the bleach ROI read out as a function of time. ${ }^{[85]}$ Within the observation time lipids showed full mobility and proteins a mobile fraction of $70 \pm 21 \%$. These values are not quantitative mobile fractions, as only the mobile parts of molecules are imaged in this setup. The mean, normalized, corrected time resolved fluorescence recovery from indirect FRAP experiments was then plotted against recovery curves obtained from finite element simulations derived using different diffusion coefficients for the s-PSM (Figure 4.7 C, D). Comparing simulated and experimental recovery curves lead to the estimated diffusion coefficients of $D_{\text {DPPE }}(\mathrm{s}-\mathrm{PSM})=2 \pm 1 \mu \mathrm{m}^{2} \mathrm{~s}^{-1}$ and $D_{\triangle \mathrm{N} 49}(\mathrm{~s}-\mathrm{PSM})=1.0 \pm 0.5 \mu \mathrm{m}^{2} \mathrm{~s}^{-1} \cdot{ }^{[95]}$ 

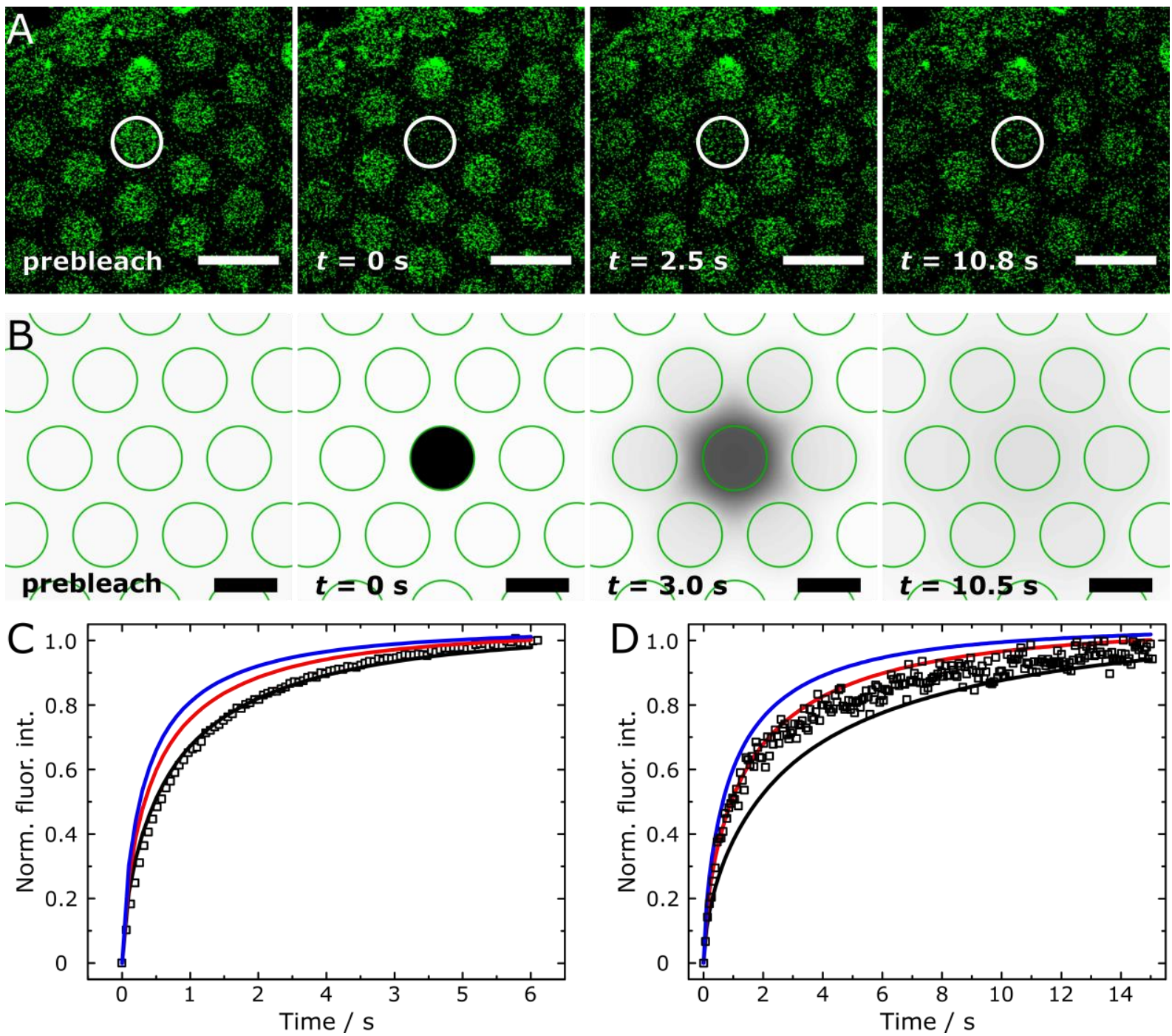

Figure 4.7 Indirect FRAP experiments and finite element simulations to determine the diffusion coefficient of lipids and proteins inside the s-PSM (DOPC/POPE/POPS/cholesterol/Atto390 DPPE; 5/1.95/1/2/0.05 (n/n), nominal p/l 1:250) (A) Fluorescence micrographs of an indirect FRAP experiment on PSMs $\left(d_{\text {pore }}=5 \mu \mathrm{m}\right)$. $\Delta$ N49-Atto488 fluorescence is bleached inside the white ROI $(r=2.2 \mu \mathrm{m})$ and fluorescence intensity was monitored over time. Scale bars $=10 \mu \mathrm{m}$. (B) Snapshots of a simulated indirect FRAP experiment obtained from finite element simulations. Fluorescence intensity is bleached inside the red ROI $(r=2.2 \mu \mathrm{m})$ and fluorescence recovery was monitored over time. (C) Averaged, corrected, normalized fluorescence recovery curve obtained from bleaching Atto390 DPPE labeled f-PSMs $\left(N=33\right.$, open squares). Simulated recovery curves with $D_{\text {DPPE }}$ (sPSM) $=2$ (black line), 3 (red line), and 4 (blue line) $\mu \mathrm{m}^{2} \mathrm{~s}^{-1}$ with fixed $D_{\text {Lipid }}$ (f-PSM) $=7.7 \mu \mathrm{m}^{2} \mathrm{~s}^{-1}$. (D) Averaged, corrected, normalized fluorescence recovery curve obtained from bleaching $\Delta$ N49-Atto488 containing f-PSMs $\left(N=33\right.$, open squares). Simulated recovery curves with $D_{\Delta \mathrm{N} 49}(\mathrm{~s}-\mathrm{PSM})=0.5$ (black), 1 (red), and $1.5($ blue $) \mu \mathrm{m}^{2} \mathrm{~s}$ ${ }^{1}$ and $D_{\text {Protein }}(\mathrm{f}-\mathrm{PSM})=3.4 \mu \mathrm{m}^{2} \mathrm{~s}^{-1}$. 


\subsection{Single-vesicle content release assay}

The fusion mechanisms and kinetics of neurotransmitter filled vesicles with the target presynaptic membrane were studied in an in vitro model system based on content labeled proteo-LUVs and lipid labeled PSMs. The v-SNARE syb 2 was reconstituted into LUVs filled with sulforhodamine $\mathrm{B}$ and the t-SNARE acceptor complex $\triangle \mathrm{N} 49$, comprised of SNAP25a, syx 1A (aa 183-288) and syb 2 (aa 49-96), reconstituted into GUVs labeled with Atto655 DPPE. GUVs were then spread on porous substrates with a pore diameter of $1.2 \mu \mathrm{m}$ to form PSMs, that exhibit a narrower pore rim than substrates with pore sizes of e.g. $5 \mu \mathrm{m}$. SRB filled proteo-LUVs were added on top of the PSM to monitor their docking and fusion process on a single-vesicle level. Measurements were performed by simultaneously reading out vesicle and target membrane fluorescence to get new insights in the interplay of fusion pore formation and lipid mixing in the process of SNARE-mediated membrane fusion. High time resolution and large scale statistical analysis enabled the quantification of key fusion kinetics and complex fusion pathways.

\subsubsection{Docking and fusion of LUVs with PSMs}

Syb 2 was reconstituted into content labeled LUVs and $\Delta$ N49-complex into GUVs as described in Chapter 3.2.4. Prior to single-vesicle fusion experiments, SNARE-activity was tested in a bulk content release assay as described in Chapter 4.1. PSMs labeled with Atto655 DPPE were prepared as described in Chapter 3.2.5, a membrane patch focused at the spinning disc confocal microscope (SDCM), and image acquisition started with $48 \mathrm{fps}$ over $6.94 \mathrm{~min}$. LUVs filled with SRB were added on top of the patch via a microinjection unit ensuring uniform vesicle addition between individual measurements and docking and fusion of vesicles with the target membrane was monitored by simultaneously recording SRB and Atto655 DPPE fluorescence intensity.

SNARE-specific docking of LUVs to the PSM was proven by blocking the syb 2 binding site of the $\Delta$ N49-complex prior to protein reconstitution. To achieve this, $\Delta N 49$-complex was incubated with syb 2 (aa 1-96) which is known to displace the small syb 2 fragment (aa 49-96) from the acceptor complex, occupying the SNARE-binding site, and rendering it non-fusogenic. ${ }^{[51]}$ Proteo-GUV formation was carried out as usual and no docking of proteo-LUVs was observed to the PSMs containing the blocked acceptor complex.

An exemplarily fluorescence micrograph of LUVs docked to the PSM is shown in Figure 4.1 B. Fluorescence intensity of the Atto655 DPPE labeled membrane (false colored in green) is only visible inside the freestanding part of the PSM (f-PSM). Fluorescence on the solid 
supported part (s-PSM) is not detectable due to the close proximity to the underlying gold surface covering the pore rim which is known to efficiently quench fluorescence intensity below $15 \mathrm{~nm}$ distance. ${ }^{[93,99]}$ LUVs docked to the s-PSM (Figure $4.1 \mathrm{~B}$, false colored in magenta) are, however, visible since they are sufficiently large for the dye-molecules inside the vesicle to exit the quenching regime of the gold-surface. Vesicles docked to the s-PSM exhibit higher fluorescence intensity comparted to vesicles docked to the f-PSM due to a maximal enhancement of fluorescence intensity of gold at distances of $\sim 40 \mathrm{~nm} \cdot{ }^{[93]}$ In total, docking of 1623 vesicles of 7 different preparations and 68 individual membrane patches was detected manually and semi automatically as described in Chapter 3.3.4. The vast majority of vesicles ( $N=1609$ ) docked to the s-PSM in close proximity to the neighboring f-PSM and remained immobile during the whole observation time without detachment from the membrane, while only 14 vesicles docked mobile to the f-PSM. This leads to $1 \%$ of vesicles that docked to or fused with the f-PSM, a part of the PSM that covers $~ 40 \%$ of the surface. In the following, vesicles were separated into two populations: those that docked to the s-PSM and those that docked to the f-PSM. While vesicles docked to the f-PSM are discussed in more detail in Chapter 4.4.6 the following chapters will focus on the docking and fusion behavior of the 1609 vesicles in contact with the s-PSM.

The evaluation of the docking behavior revealed a large variation in the total number of docked vesicles $\left(N_{\text {docked }}\right)$ between the 68 different membrane patches. To evaluate whether this is due to variations in patch sizes the total surface area of all 68 membrane patches $\left(A_{\text {patch }} / \mu \mathrm{m}^{2}\right)$ was extracted and plotted against $N_{\text {docked }}$. The results shown in Figure 4.8 indicate that larger patch sizes only partially correlate with higher numbers of docked vesicles. As a quantitative measure, the Pearson correlation coefficient $\rho(A, B)$ of the two populations $A$ and $B$ was calculated using Equation (4-2).

$$
\rho(A, B)=\frac{1}{N-1} \sum_{i=1}^{N}\left(\left(\overline{\frac{A_{l}-\mu_{A}}{\sigma_{A}}}\right)\left(\frac{B_{i}-\mu_{B}}{\sigma_{B}}\right)\right)
$$

Here, $N$ is the amount of scalar observations, $\mu_{A}, \sigma_{A}, \mu_{B}$, and $\sigma_{B}$ the mean and standard deviation of $A$ and $B$. As values of +1 and -1 show high positive or negative correlation of the two populations and values $\sim 0$ no correlation, the resulting value of $\rho(A, B)=0.258$ leads to the conclusion that larger patch sizes do not necessarily result in higher amounts of docked vesicles. 
Thus, to account for different patch sizes, the number of proteo-LUVs docked to the PSMs $N_{\text {docked }}$ was normalized by the patch size $A_{\text {patch }}$ resulting in an average docking efficiency of $0.43 \pm 0.56$ vesicles per $\mu \mathrm{m}^{2}$ with the standard deviation as error. The very broad distribution of docking efficiencies is likely to originate from the varying amount of $\Delta \mathrm{N} 49$ reconstituted into the PSM. As each membrane patch originates from a different GUV, the large differences of p/l ratios inside GUVs $(R=26 \pm 24 \%)$ as described in Chapter 4.2 will lead to varying acceptor complex densities inside the target membrane.

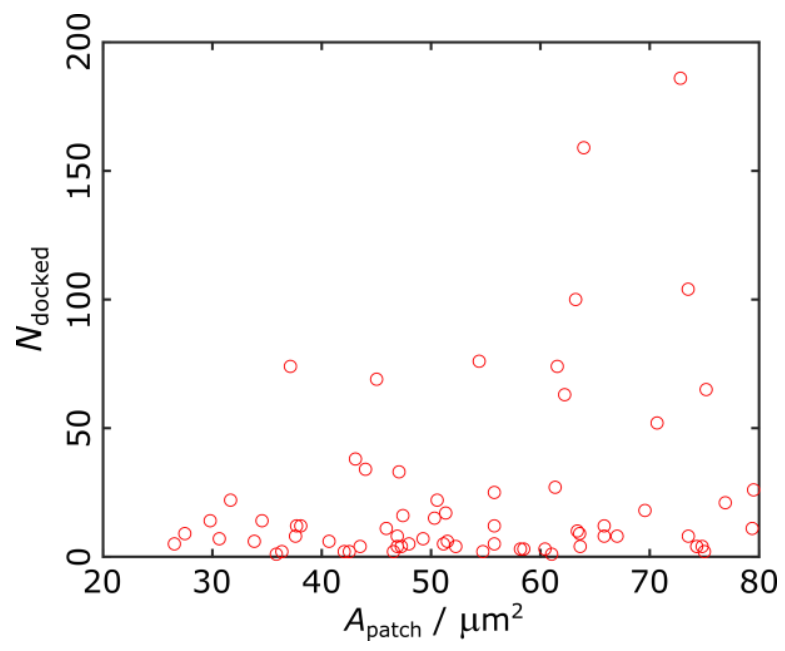

Figure 4.8 Scatterplot of the number of docked vesicles $N_{\text {docked }}$ against the total surface area $A_{\text {patch }}$ of the respective membrane with a correlation coefficient of $\rho=0.258$.

All docked vesicles were tagged manually with a stationary region of interest (ROI, Figure 4.9 A) in ImageJ and further evaluated as described in Chapter 3.3.4. For this, the vesicular ROI was transferred to the membrane-channel, fluorescence intensities of both ROIs were read out, background and crosstalk corrected, and plotted as a function of time (Figure $4.9 \mathrm{~B}$ ). When the vesicle docked to the s-PSM fluorescence intensity increased inside the ROI and fusion is detected as a drop of this intensity. In this example content release is complete as the intensity time trace drops to baseline level. The lack of an increase in target membrane fluorescence (Figure $4.9 \mathrm{~B}$, green line) is caused by no detectable diffusion of lipid dye molecules over the fusion stalk into the 3D structure of the liposome. As the vesicle is docked to the s-PSM such a diffusion would result in a dequenching of s-PSM fluorescence, since lipid molecules would exit the quenching regime of the underlying gold surface and to a certain extent enter the regime were fluorescence intensity is enhanced. ${ }^{[93,106]}$ This suggests that the very rapid content release kinetic after fusion pore formation of only several frames and the accompanied collapse of the vesicle into the target membrane occur too fast for lipids to diffuse into the 3D structure of the vesicle. This type of fusion behavior is further referred to as full release. 


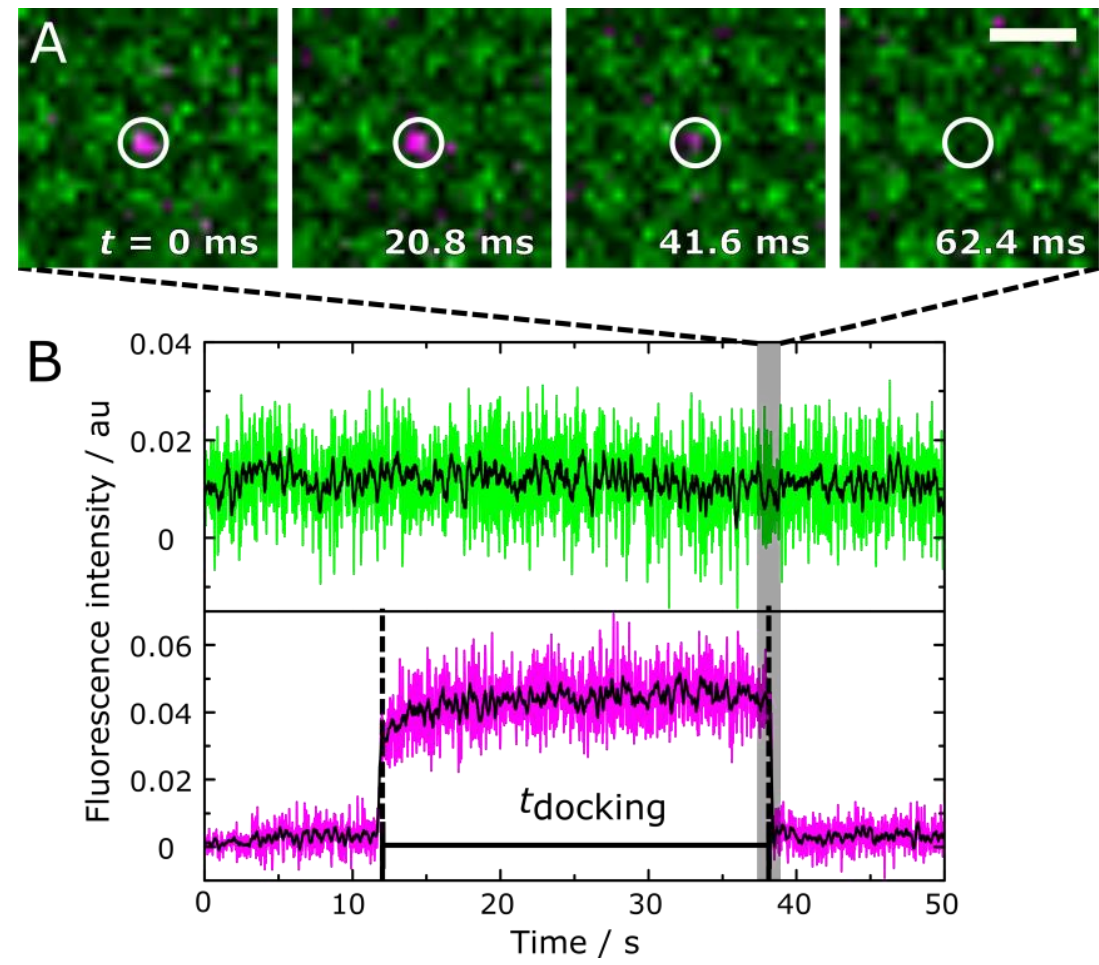

Figure 4.9 Typical single-vesicle content release event. (A) Time lapse fluorescence images of a SRB-filled syb 2 doped LUV (false colored in magenta) docked to the Atto655 DPPE labeled $\triangle$ N49 containing s-PSM (false colored in green, $t=0 \mathrm{~ms}$ ). Complete content release upon fusion pore formation occurs in the matter of 3 frames. The region of interest (ROI) was used to read out SRB and Atto655 DPPE fluorescence intensity as a function of time. Scale bar $=2 \mu \mathrm{m}$. (B) Fluorescence intensity time traces of vesicle (magenta) and membrane (green) fluorescence obtained from the ROI shown in (A) with smoothed data points (black). The time between docking $(t \approx 12 \mathrm{~s})$ until fusion pore formation $(t \approx 38 \mathrm{~s})$ is defined as the docking time $t_{\text {docking. }}$.

As discussed in Chapter 1.2.2, a solid support underneath the docked vesicle can influence its fusion behavior to an extent where it most likely bursts its content into the solution above the membrane rather than transferring it across the membrane due to fusion pore formation (Chapter 1.2.2). ${ }^{[74]}$ Since LUVs in this work docked primarily to the solid supported s-PSM it was necessary to investigate if a decrease in SRB-fluorescence as described in Figure 4.9 B can be referred to as fusion pore formation and did not occur due to bursting of the vesicle. Indeed, in the present work the $2^{\text {nd }}$ aqueous compartment underneath the f-PSM next to the docking site of the vesicle could uptake the released content which in some cases can be directly visualized (Figure 4.10 A). Due to the high temporal resolution the content of the vesicle that was docked to the s-PSM (Figure 4.10 A ROI 1, I) was detected after fusion pore formation underneath the f-PSM (Figure 4.10 A ROI 2, II) followed by rapid diffusion out of focus (Figure 4.10 A ROI 2, III). This process can also be seen in the fluorescence intensity time trace of ROI 1 and 2 (Figure $4.10 \mathrm{C}$ ) where the released content leads to a spike in fluorescence intensity underneath the f-PSM. Simultaneously, fluorescence of vesicular ROI 1 drops down to baseline level and shows no such spike in intensity upon content release. However, if a vesicle is bursting its 
content into the solution above the membrane (Figure 4.10 B), reading out fluorescence of the vesicular ROI leads to a sharp spike in intensity upon content burst. This spike is a result of the dequenching of SRB dye when it exits the interior of the vesicle and can be detected as the dye is released into the solution directly above the vesicular ROI. Thus, this spike in vesicular fluorescence intensity was used to efficiently separate fusion pore formation and vesicle burst events of 1609 docked vesicles. In total, $52 \%$ of vesicles $(N=840)$ fused with the s-PSM and only $0.003 \%$ burst $(N=6)$, making vesicle bursting an event that can be neglected for the model system of PSMs as the target membrane in SNARE-mediated fusion.
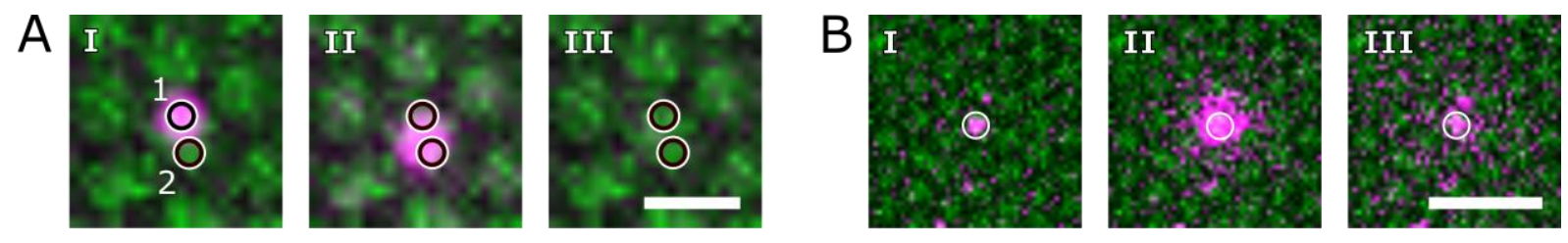

C
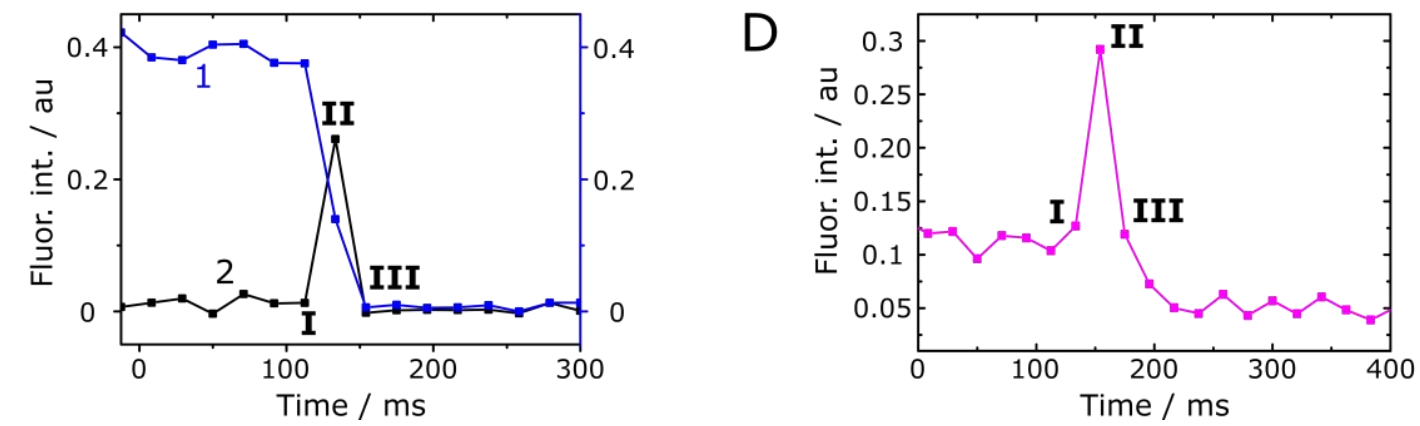

Figure 4.10 Proof for direction of content release (A) Fluorescence intensity micrographs of a fusing vesicle (magenta) that transfers its content across the PSM into the $2^{\text {nd }}$ aqueous compartment. Scale bar $=2 \mu \mathrm{m}$. (B) Fluorescence intensity micrographs of a vesicle (magenta) bursting its content into the solution above the membrane. Scale bar $=2 \mu \mathrm{m}$. (C) Fluorescence intensity time traces obtained by read out of ROI 1 and 2 seen in (A). The vesicle docked to the s-PSM until time point I to then fuse and transfer its content into the aqueous space underneath the adjacent f-PSM (II). This leads to an increase in fluorescence intensity in ROI 2 (II) followed by rapid diffusion of the dye (III). (D) Fluorescence intensity time trace obtained by read out of the ROI shown in (B). The vesicle stays docked to the s-PSM (I) until the burst of content into the solution above the membrane results in a spike in fluorescence intensity (II) followed by rapid diffusion of the content into the bulk solution.

\subsubsection{Docking times of proteo-LUVs}

After differentiating between fusion pore formation and vesicle bursting the time between docking and fusion of 840 proteo-LUVs (Figure $4.9 \mathrm{~B}$ ) was extracted using the semi-automatic Matlab script described in Chapter 3.3.4. This so called docking time $t_{\text {docking }}$ can be an important measure for the overall kinetics of the fusion process. The histogram of the docking time distribution is shown in Figure 4.11 with the bin width calculated using Equation (4-3).

$$
\text { Bin width }=2 \frac{\operatorname{IQR}(x)}{\sqrt[3]{n}}
$$


Here, $\operatorname{IQR}(x)$ is the interquartile range of the data with its total number of individual data points $n$. A mono-exponential decay function (Equation (4-4)) was then fit to the data, resulting in an average docking lifetime of $\tau_{\text {docking }}=65 \pm 4 \mathrm{~s}$. The rate constant $k$ can be calculated as the reciprocal of $\tau_{\text {docking }}$ and results in $k=0.0154 \mathrm{~Hz}$.

$$
y(x)=-a \cdot \exp (-k x)
$$

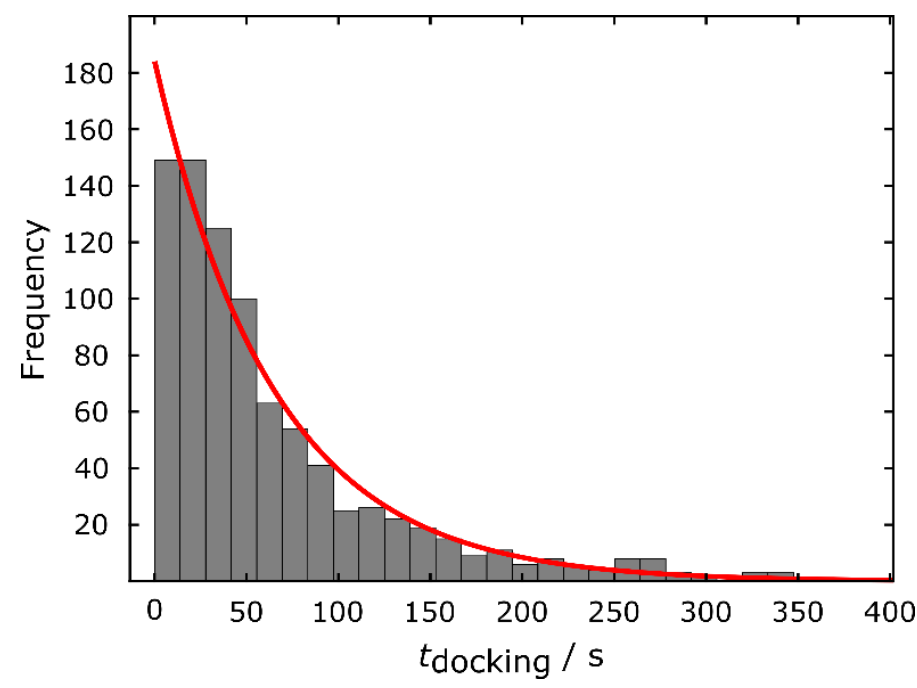

Figure 4.11 Docking time distribution obtained by extracting $t_{\text {docking }}$ from $N=840$ fusion events. A mono-exponential decay function (red) was fit to the data with an average lifetime $\tau_{\text {docking }}=65 \pm 4 \mathrm{~s}$.

The docking time distribution contains data from 68 different time series recorded from vesicles fusing to individual membrane patches. As the docking efficiency to these patches varied drastically $\left(0.43 \pm 0.56\right.$ vesicles $\left.\mu \mathrm{m}^{-2}\right)$, it had to be examined whether it influences the mean docking time of vesicles fusing with the respective PSM. Thus, the docking efficiency was plotted against the mean docking time at the respective membrane patch (Figure 4.12 A). As highlighted by the envelopes of the data, mean docking times converge towards the global average docking lifetime (grey horizontal line) of $\tau_{\text {docking }}=65 \mathrm{~s}$. The data scatters significantly for lower docking efficiencies, as outliers have a higher impact on the mean docking time due to the lower total number of fusing vesicles. Supported by the calculated correlation coefficient of $\rho=0.137$ it can be assumed that larger amounts of docked vesicles per $\mu \mathrm{m}^{2}$ do not lead to longer or shorter docking times. However, to test if docking times are influenced by the size of the fusing LUV, the mean vesicle fluorescence intensity prior to fusion was extracted from fluorescence intensity time traces. It has to be stressed that no quantitative relation between vesicle intensity and vesicle diameter can be drawn. While all experiments were performed with the same microscopy settings and a constant SRB concentration inside the vesicle population 
can be assumed, differently sized vesicles are influenced in their emitted fluorescence non-linearly by the gold functionalization. This is due to the fact that the gold surface quenches fluorescence at distances $<15 \mathrm{~nm}$ while it increases the fluorescence until a distance of $\sim 80 \mathrm{~nm}$ with a maximum increase at $40 \mathrm{~nm}$ distance. Nevertheless, mean vesicle intensity can and will be used as a relative measure for vesicles sizes. The scatter plot of vesicle intensity against $t_{\text {docking }}$ (Figure $4.12 \mathrm{~B}$ ) together with the calculated correlation coefficient of $\rho=0.0727$ indicate that larger vesicle sizes do not lead to longer or shorter docking times.
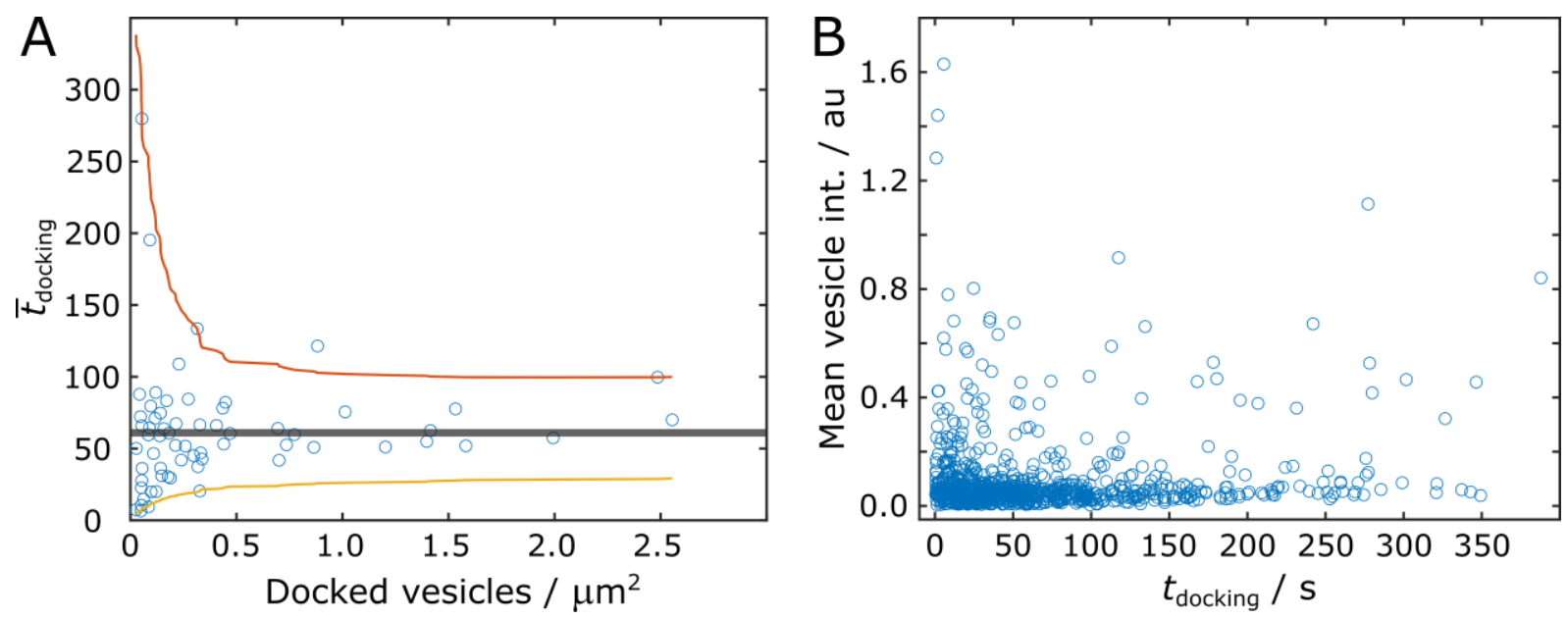

Figure 4.12 (A) Scatter plot of docking efficiencies to PSMs against mean docking times of the respective membrane patch. The data converges for large amounts of docked vesicles per $\mu \mathrm{m}^{2}$ to the global average docking lifetime of $65 \mathrm{~s}$ (horizontal grey line) highlighted by the envelopes of the population. Correlation coefficient $\rho=0.137$. (B) Scatter plot of docking times against mean vesicle intensity prior to content release. The populations show no direct correlation supported by the correlation coefficient of $\rho=0.0727$.

\subsubsection{Fusion pathways of vesicles}

As described in Figure 1.3 often a straight forward fusion pathway from vesicle docking to vesicle collapse into the target membrane after fusion pore formation is discussed to be mediated by the SNARE machinery. However, complex 3D postfusion structures have been recorded in vivo with vesicles collapsing delayed into the target membrane after fusion pore formation or retaining their so called $\Omega$-shape. ${ }^{[109]}$ In this work, simultaneous detection of lipid diffusion over the fusion stalk from the s-PSM into the docked LUV and content release through the fusion pore allowed to monitor and quantify different fusion pathways.

Schematic illustrations of a vesicle fusing with the PSM showing visible lipid mixing and the resulting theoretical fluorescence intensity time trace is illustrated in Figure 4.13. While the fluorescence intensity time trace of the dye SRB provides information about the time point of fusion pore formation, merging of the outer and inner leaflet of the bilayers leads to the exchange of lipid material, also called lipid mixing. When lipids of the s-PSM diffuse into the 
membrane of the fusing vesicle they exit the quenching regime of the gold surface covering the pore rims, which leads to a drastic increase in fluorescence intensity (Figure 4.13 II, green channel). Depending on the lifetime of the 3D postfusion structure fluorescence remains constant (Figure $4.13 \mathrm{III}$ ) until the complete merging of the vesicle into the target membrane leads to a drop in intensity to baseline level (Figure 4.13 IV-V).

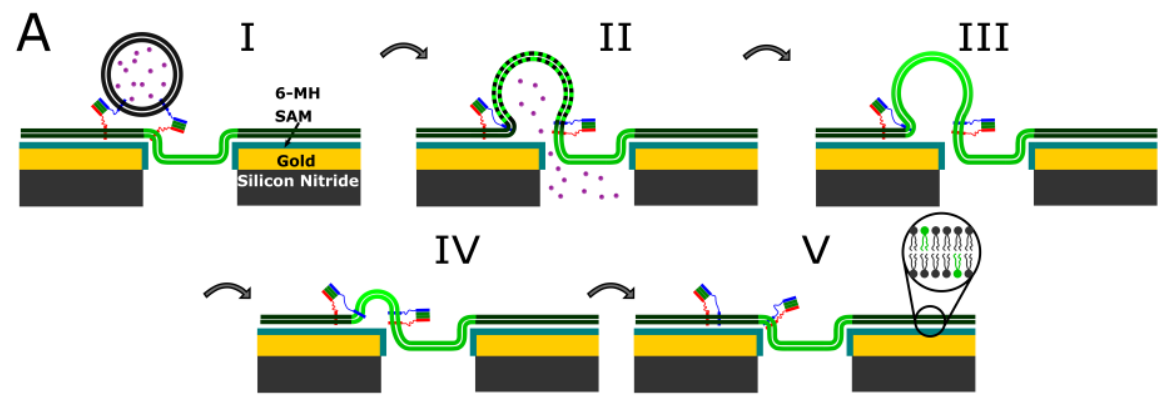

B

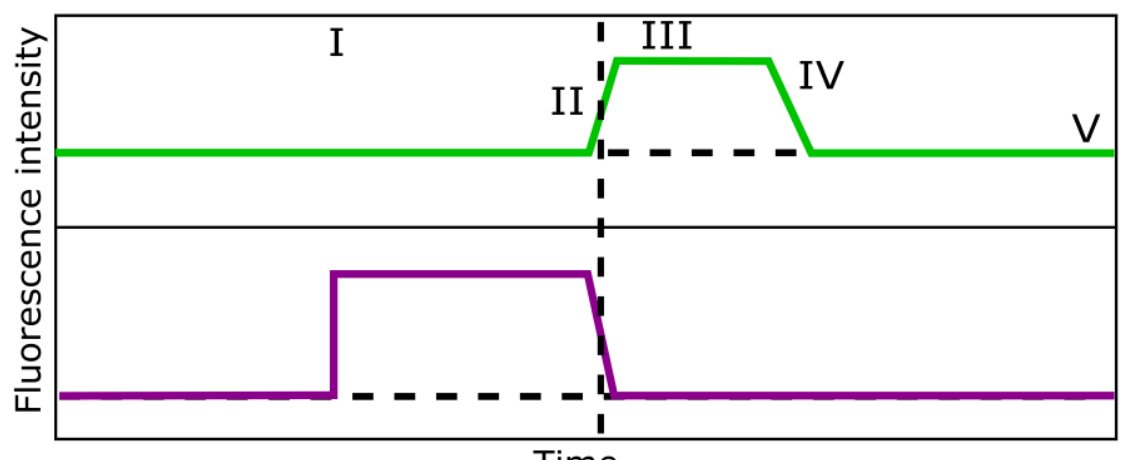

Figure 4.13 (A) Schematic illustrations of important intermediate steps of a vesicle fusing with the s-PSM with detectable diffusion of lipids into the $\Omega$-shape. (B) Respective idealized fluorescence intensity time traces of vesicle (magenta) and target membrane (green) fluorescence intensity. Docking of the vesicle (I) leads to an increase in SRB fluorescence, while s-PSM fluorescence is quenched due to the underlying gold surface. Upon fusion pore formation (II) content release leads to a decrease in SRB fluorescence. Simultaneous diffusion of lipids over a possible fusion stalk into the vesicle leads to an increase in Atto655 DPPE fluorescence as lipids dyes exit the quenching regime of the gold. The $\Omega$-shape retains its structure (III) until the onset of vesicle collapse into the s-PSM leads to the re-entering of dyes into the quenching regime and a decrease of fluorescence intensity. A complete content release and vesicle collapse is indicated by a decrease of both channels to baseline levels.

The evaluation of 447 single fusion events during which lipid mixing was observed revealed three possible fusion pathways with their final state and respective fluorescence intensity time traces shown in Figure 4.14. These pathways differ in the amount of released content and the stability of the $\Omega$-shape. An incomplete content release (Figure $4.14 \mathrm{~A}, N=202$ ), possibly due to a metastable fusion pore, resulted in a remaining Atto655 DPPE intensity above baseline level until the end of the observation time. Due to the remaining content inside the vesicle, the 3D structure of the vesicle is prevented from collapsing completely into the target membrane. In the case of a full release the vesicle either remained in its 3D postfusion structure (Figure 
4.14 $\mathrm{B}, N=138$ ) or collapsed completely back into the target membrane (Figure $4.14 \mathrm{C}$, $N=107)$. Stalled hemifusion, where only the two outer leaflets mix without pore formation, was rarely.
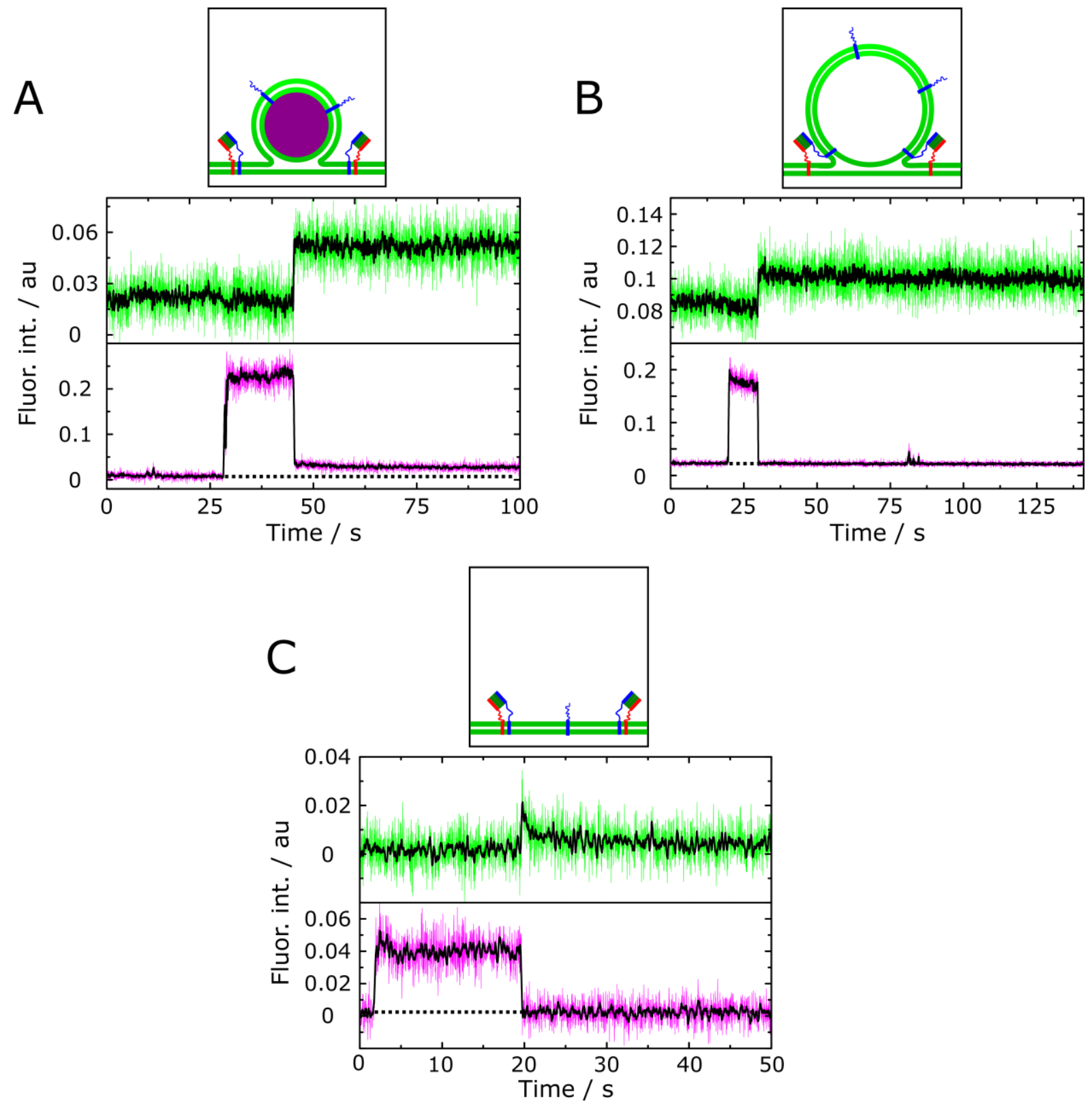

Figure 4.14 (Upper panel) Schematic (post)fusion structures of different fusion pathways with (lower panel) respective fluorescence intensity time traces of fusing vesicles (magenta) with the planar s-PSMs (green) with smoothed data (black). (A) Incomplete content release with a remaining three-dimensional (3D) postfusion structure. (B) Complete content release with a remaining stable 3D postfusion structure. (C) Complete content release with an unstable $3 \mathrm{D}$ postfusion structure.

The maximum level of increase in s-PSM fluorescence during lipid mixing is likely to be dependent on the size of the vesicle. A larger vesicle would lead to more fluorophores inside the confocal volume of the objective, as the z-confocality of 1-2 $\mu \mathrm{m}$ is larger than the vesicle diameter. To test this, mean vesicle intensities of LUVs fusing with visible lipid mixing were 
plotted against the maximum s-PSM fluorescence intensity of the $\Omega$-shape (Figure 4.15). Vesicles with a larger mean fluorescence intensity show a strong trend to higher maximum s-PSM intensities during lipid mixing with an extracted correlation coefficient of $\rho=0.6022$.

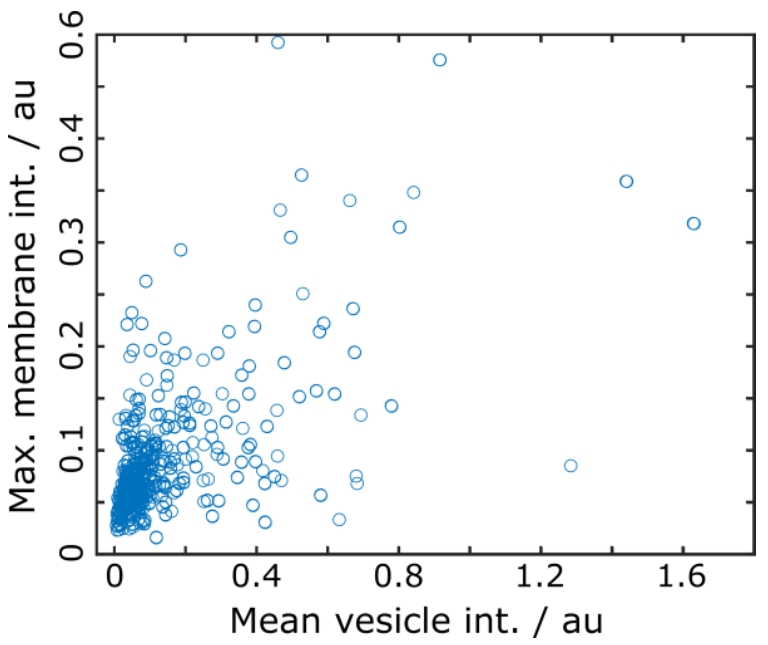

Figure 4.15 Scatter plot of mean vesicle intensities against maximum s-PSM intensities during lipid diffusion into the $3 \mathrm{D}$ postfusion structure of the vesicle. The populations show strong positive correlations with a correlation coefficient of $\rho=0.6022$.

In summary, vesicles can undergo fusion with or without visible lipid mixing (Figure 4.9 and Figure 4.14) with a fusion efficiency of $52 \%$. Figure 4.16 depicts all observed fusion pathways as well as their respective schematic fluorescence intensity time traces which were used to differentiate between the event types. To quantify the likelihood of each fusion pathway, the weighted mean $\bar{x}$, which describes the relative occurrence of each fusion pathway, was calculated using Equation (4-5).

$$
\bar{x}=\frac{1}{\sum_{i=1}^{n} w_{i}} \sum_{i=1}^{n} x_{i} \cdot w_{i}
$$

Here, $n=68$ is the number of independent experiments (individual membrane patches) and $x_{\mathrm{i}}$ the relative amount of each fusion pathway occurring on these patches. $W_{\mathrm{i}}$ is the statistical weighting factor defined as the number of docked vesicles per $\mu \mathrm{m}^{2}$. As the docking efficiency is interpreted as a function of fusion active acceptor complex inside the PSM, the weighting factor will account for large variations in $\triangle \mathrm{N} 49$-complex densities inside the PSM and average out possible outliers originating from membrane patches with low docking efficiencies. Weighted standard deviations $S D$ were calculated using Equation (4-6). 


$$
S D=\sqrt{\frac{\sum_{i=1}^{n} w_{i}}{\left(\sum_{i=1}^{n} w_{i}\right)^{2}-\sum_{i=1}^{n} w_{i}^{2}} \cdot \sum_{i=1}^{n}\left(x_{i}-\bar{x}\right)^{2} \cdot w_{i}}
$$

Overall, $24 \pm 11 \%$ of vesicles fused with complete content release without detectable lipid diffusion into the 3D structure of the vesicle. The remaining $28 \pm 16 \%$ of fusing vesicles showed lipid diffusion over a possible fusion stalk with the majority of vesicles showing a stable $\Omega$-shape. Only $13 \pm 9 \%$ of vesicles showed incomplete content release (25\% of fusing vesicles). The total number $N$ of vesicles fusing over a certain fusion pathway used to extract the aforementioned percentages and create Figure 4.16 is summarized in Table 4-1.

Table 4-1 Total number $N$ of vesicles fusing via a certain fusion pathway used to determine relative occurrences.

\begin{tabular}{l|l} 
Fusion pathway & $N$ \\
\hline Full release & 393 \\
Incomplete release & 202 \\
Full release \& vesicle collapse & 107 \\
Stable 3D postfusion structure & 138 \\
Bursting vesicle & 6 \\
No fusion pore formation & 763
\end{tabular}




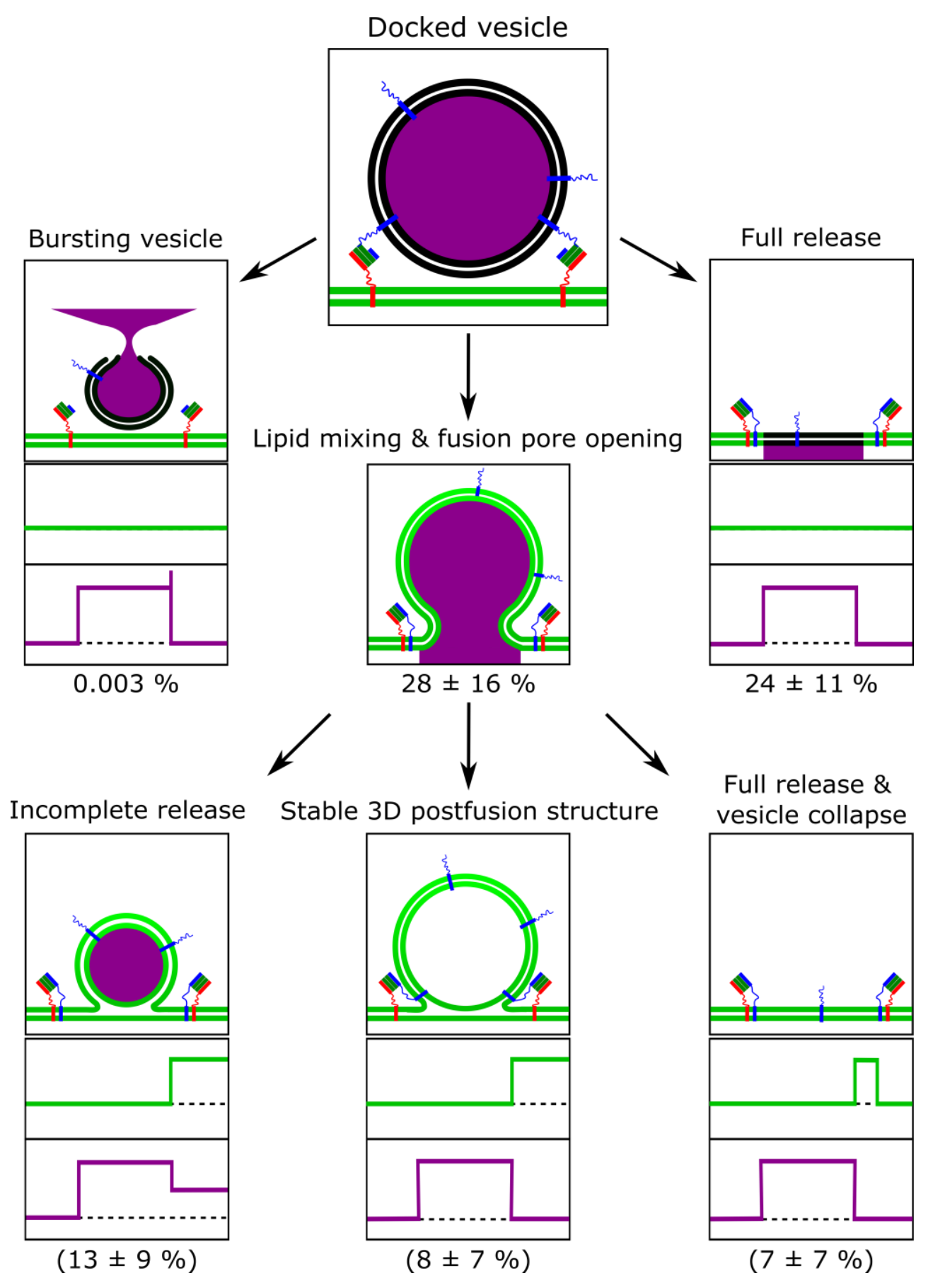

Figure 4.16 Summary of possible fusion pathways monitored during SNARE-mediated membrane fusion of LUVs with s-PSMs. In total, 1609 vesicles docked to the s-PSM of which 840 (52\%) proceeded to fusion and $75 \%$ of these LUVs released the content dye completely after fusion pore formation. $24 \pm 11 \%$ of vesicles fused without detectable lipid mixing while in $28 \pm 16 \%$ of events lipid diffusion over a fusion stalk was detected concomitant to content release. Vesicle bursting was recorded in only $0.003 \%$ of the vesicle population.

While complex 3D post fusion structures were also described and quantified in vivo, the underlying mechanisms that lead to different fusion pathways are unclear. ${ }^{[109]}$ One possibility might be that higher acceptor complex densities, which is reflected in higher docking efficiencies, might influence the fusion pathway probabilities. To test this, correlation coefficients between docking efficiencies of LUVs to different PSM-patches and the 
non-weighted probability of a certain fusion pathway were calculated using Equation (4-2). The results are summarized in Table 4-2 and an exemplarily scatter plot shown in Figure 4.17. For low docking efficiencies the amount of vesicles fusing without visible lipid mixing varies significantly and converge to the global weighted mean value of $24 \%$ (Figure 4.17 , grey horizontal line). This is the case for each individual fusion pathway and is reflected in correlation coefficients close to 0 , ranging from $\rho=-0.0918$ for vesicles that show no fusion pore formation to $\rho=0.2382$ for vesicles that show a stable 3D postfusion structure including a complete release. The docking efficiency and thus the amount of accessible acceptor complex inside the s-PSM seems to have no influence on the fusion pathway of the vesicle.

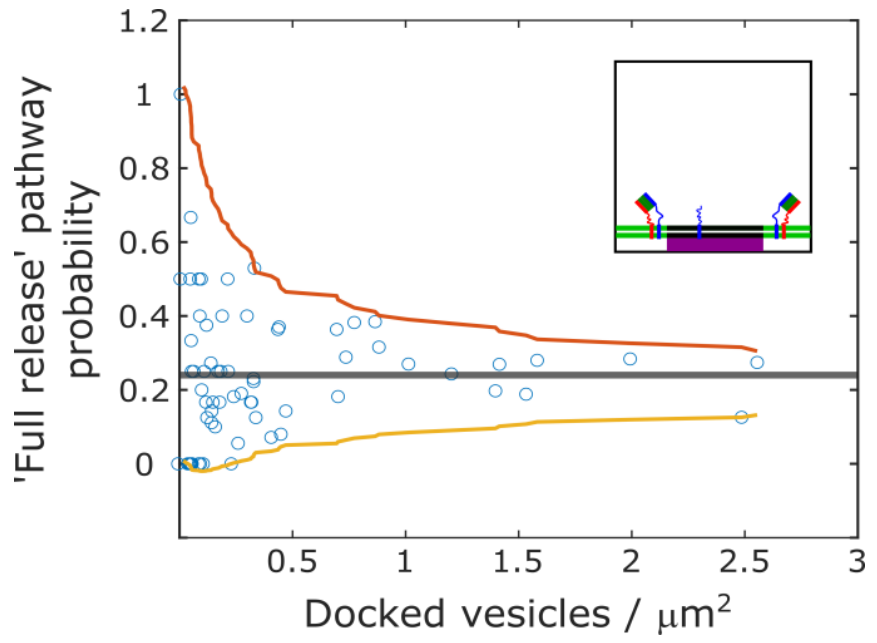

Figure 4.17 Scatter plot of docking efficiencies to PSMs against the probability of the vesicles undergoing fusion via the 'full release' pathway at the respective membrane patch. The data converges for large amounts of docked vesicles per $\mu \mathrm{m}^{2}$ to the global weighted mean of $24 \%$ (horizontal grey line) highlighted by the envelopes of the population. Correlation coefficient $\rho=0.0355$.

Table 4-2 Summary of correlation coefficients of occurrence of fusion pathways obtained from independent experiments with the respective docking efficiency of these experiments.

\begin{tabular}{l|l} 
Fusion pathway & Correlation coefficient \\
\hline Full release & 0.0355 \\
Incomplete release & -0.0102 \\
Full release \& vesicle collapse & -0.042 \\
Stable 3D postfusion structure & 0.2382 \\
No fusion pore formation & -0.0918
\end{tabular}

Besides differences in docking efficiencies to each PSM another factor that varied in the single-vesicle fusion experiments was the size of the fusing LUV, since vesicle diameters 
produced as described in Chapter 3.2.4 were proven to show large distributions. ${ }^{\left[{ }^{[6]}\right.}$ By plotting histograms of mean vesicle fluorescence intensities for each fusion pathway (Figure 4.18) it was tested if different vesicles sizes influenced the fusion pathway of LUVs. Since, as discussed in Chapter 4.4.2, the mean fluorescence is not a quantitative measure for vesicle sizes the data was not fitted or any mean intensity values extracted. However, when looking at the vesicle intensity distributions, certain fusion pathways show a trend to higher vesicle intensities. Only $2 \%$ of vesicles fusing with complete content release and without visible lipid mixing (Figure $4.18 \mathrm{~A}$ ) show fluorescence intensities over $0.2 \mathrm{au}$. On the other hand, $7 \%$ of vesicles that release the content completely with visible lipid diffusion into the 3D structure (Figure 4.18 C, D) show mean intensities over $0.2 \mathrm{au}$. This fraction of larger vesicles inside the population increases to $26 \%$ for LUVs that fused with an incomplete content release. Thus, larger vesicles tend to fuse with a visible $\Omega$ shape and the population of LUVs that release the content incompletely has the highest amount of large vesicles.
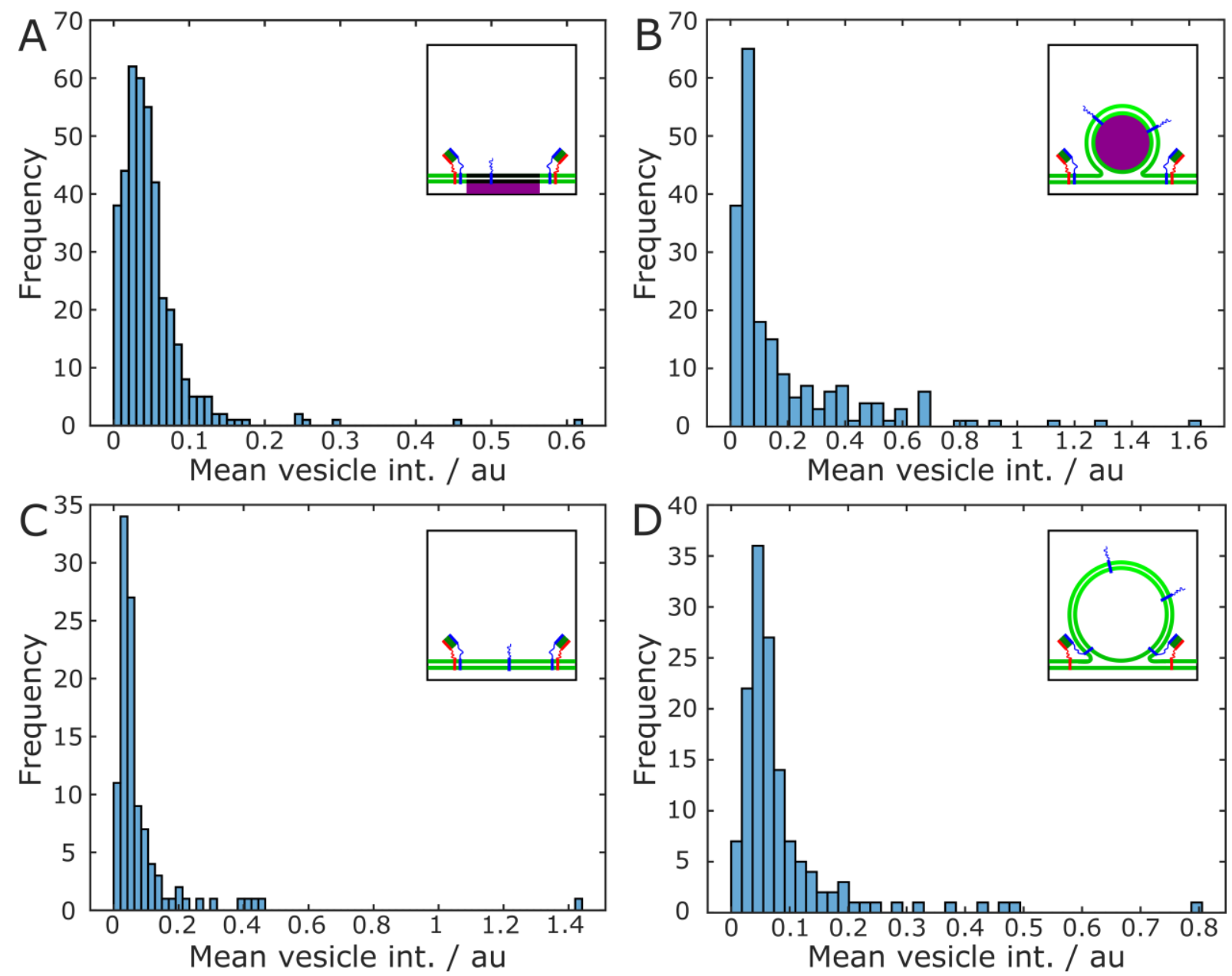

Figure 4.18 Histograms of mean vesicle intensities prior to fusion pore formation against each detected fusion pathway. (A) Full release pathway $(N=393)$ contain vesicle intensities of up to $\sim 0.6$ au with $2 \%$ of the population exhibiting intensities over 0.2. (B) $26 \%$ of LUVs fusing incompletely $(N=202)$ exhibit intensities over 0.2 au. 
$7 \%$ of LUVs showing a full release and vesicle collapse $(N=107)(\mathrm{C})$ as well as a stable 3D postfusion structure $(N=138)(\mathrm{D})$ exhibit intensities over 0.2 au.

\subsubsection{Flickering fusion pores}

In vivo studies showed that the release of neurotransmitters in SNARE-mediated membrane fusion can occur in dynamic openings and closings of the fusion pore. ${ }^{[110-114]}$ This behavior, which is referred to as a flickering fusion pore, was also observed during this work which only used the minimal fusion machinery to trigger the fusion of content labeled LUVs with PSMs. Figure 4.19 A highlights important time points of the fusion pathway of a vesicle fusing with the s-PSM in two consecutive content release events with exemplarily fluorescence intensity time traces depicted in Figure 4.19 B. Vesicle docking to the target membrane (I), as discussed in Chapter 4.4.1, leads to an increase in detected SRB fluorescence (Figure 4.19 B, magenta, lower panel) while s-PSM fluorescence remains quenched. SNARE-mediated fusion of the two outer leaflets (II) leads to the diffusion of Atto655 DPPE molecules into the $\Omega$-shape of the vesicle and results in the dequenching of s-PSM fluorescence. Simultaneously, the fusion pore opens and content molecules are transferred across the target membrane and released into the $2^{\text {nd }}$ aqueous compartment underneath the PSM. The fusion pore rapidly closes again which leads to an incomplete first content release (III). With a likelihood of $51 \%$ the fusion pore opens again and more LUV-content is released in a $2^{\text {nd }}$ fusion event. In the case of a full release as the final state, which happens $25 \%$ of the time, vesicle fluorescence drops down to baseline level and is accompanied by a collapse of the $\Omega$-shape back into the s-PSM (IV). $8 \%$ of all vesicles have shown such a flickering fusion pore which can in some cases open and close more than 2 times. 


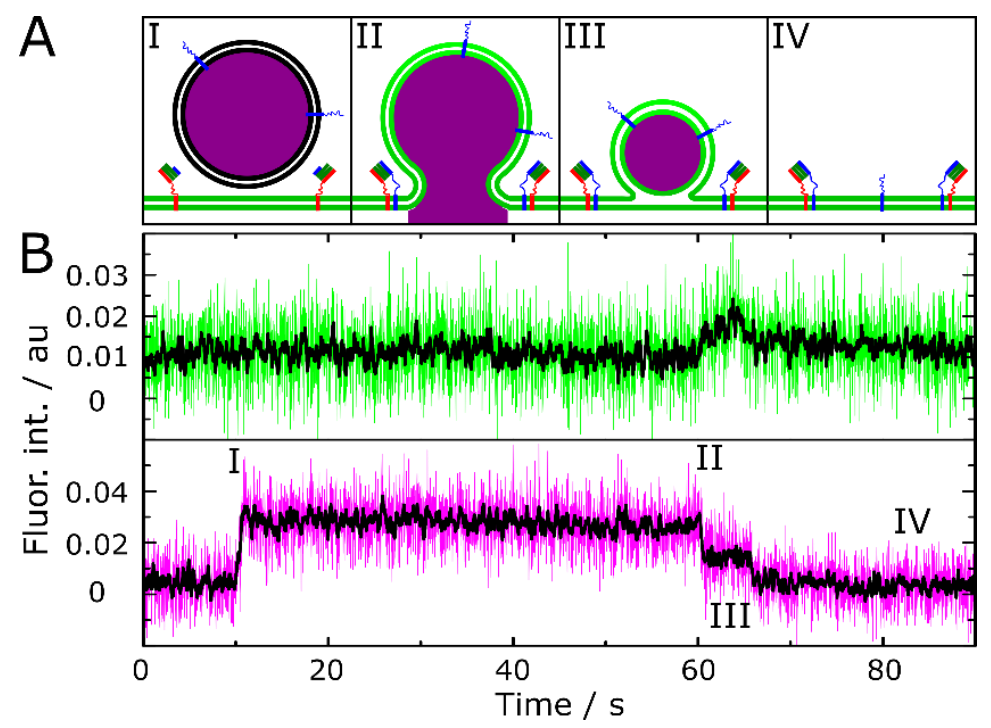

Figure 4.19 (A) Schematic illustration of key steps of a vesicle fusing with a flickering fusion pore. (B) Exemplarily fluorescence intensity time traces of the vesicular content (magenta) and s-PSM (green) with smoothed data (black). After docking to the s-PSM (I) the fusion of the two bilayers (II) leads to the diffusion of lipids from the PSM into the $\Omega$-shape of the vesicle resulting in an increase in Atto655 DPPE fluorescence (green channel). Simultaneous opening and rapid closing of the fusion pore leads to a partial content release (III). Reopening of the fusion pore with a likeliness of $51 \%$ results in a consecutive content release (IV).

To test whether the underlying mechanisms of a $2^{\text {nd }}$ fusion pore formation differ from the formation of the first fusion pore, the lag time between the $1^{\text {st }}$ and $2^{\text {nd }}$ release was extracted from $N=112$ fusion events. The histogram of lag times is depicted in Figure 4.20 and shows an exponential distribution of docking times. Fitting a mono-exponential fit (Equation (4-4)) to the data results in an average lag time of $51 \pm 10 \mathrm{~s}$. The extracted value is close to the average docking lifetime of $\tau_{\text {docking }}=65 \pm 4$ s determined for the lag time between docking and $1^{\text {st }}$ fusion pore formation (Chapter 4.4.2). Performing a Mann-Whitney U-test between the two populations failed to reject the null hypothesis at $5 \%$ significance with $\mathrm{p}=0.0657$. Thus, the two docking lifetimes do not differ significantly from each other. 


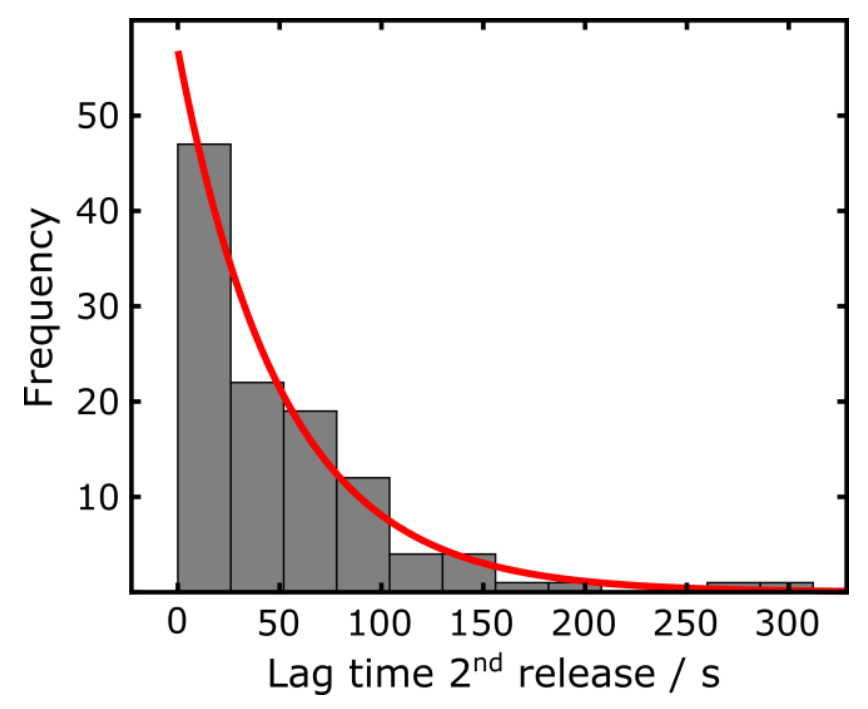

Figure 4.20 Histogram of lag times $(N=112)$ between first and second content release events of vesicles fusion via a flickering fusion pore. Fitting a mono-exponential decay to the data results in an average lag time of $51 \pm 10 \mathrm{~s}$.

\subsubsection{Kinetics of vesicle fusion}

To gather more information about the kinetics of fusion pore formation and lipid diffusion into the $\Omega$-shape, different important time points of the fusion process were determined as described in Chapter 3.3.4 (Figure 4.21 A, B). First, the starting point of lipid diffusion into the 3D structure of the vesicle (Figure 4.21 B, blue x), the onset of the collapse of the vesicle back into the s-PSM, and the end of the collapse (Figure 4.21 B, green $\mathrm{x}$ ) were extracted. Out of these values kinetic information about the time between lipid diffusion into the $\Omega$-shape and the start of the collapse into the membrane $\left(\tau_{\text {rise }}\right)$, the collapse process of the vesicle back into the membrane $\left(\tau_{\text {collapse }}\right)$, and the overall lifetime of the $\Omega$-shape $\left(\tau_{\text {rise }+ \text { collapse }}\right)$ were obtained. Cumulative distribution functions of $\tau_{\text {rise }}, \tau_{\text {collapse, }}$, and $\tau_{\text {rise+collapse }}$ from $N=234$ individual events are depicted in Figure 4.21 C-E. For $\tau_{\text {rise }}$ only a bi-exponential fit was able to properly describe the data suggesting two populations of vesicles one of which starts to collapse back into the membrane more quickly than the other. This also holds true for the collapse process of vesicles $\tau_{\text {collapse }}$ and for the overall lifetime of the $\Omega$-shape $\tau_{\text {rise+collapse. The results of fitting Equation }}$ (4-7) to the data are summarized in Table 4-3. The rise and the collapse process show very similar rate constants that result in average lifetimes of $\tau_{\text {rise }}$ of $23 \pm 2 \mathrm{~s}$ and $2.3 \pm 0.1 \mathrm{~s}$ and $\tau_{\text {collapse }}$ of $23 \pm 1 \mathrm{~s}$ and $2.4 \pm 0.1 \mathrm{~s}$. However, the correlation coefficient of the two different processes of $\rho\left(\tau_{\text {rise }} / \tau_{\text {collapse }}\right)=0.1483$ suggests that longer rise times are not accompanied with longer collapse times. Thus, the increase of fluorescence due to lipid diffusion into the 3D structure of the vesicle and the subsequent collapse of the vesicle into the target membrane happen independently from each other. Rate constants of the overall stability of the $\Omega$-shape 
result in mean lifetimes for the more stable population of $50 \pm 5 \mathrm{~s}$ and for the other vesicle population of $10 \pm 1 \mathrm{~s}$.

$$
y(x)=-a \cdot \exp \left(-k_{1} x\right)-(1-a) \cdot \exp \left(-k_{2} x\right)+1
$$

Table 4-3 Rate constants extracted from the 3D postfusion structure of vesicles.

\begin{tabular}{l|l|l} 
& $k_{1} / \mathrm{s}^{-1}$ & $k_{2} / \mathrm{s}^{-1}$ \\
\hline$\tau_{\text {rise }}$ & $0.043 \pm 0.004$ & $0.43 \pm 0.01$ \\
$\tau_{\text {collapse }}$ & $0.044 \pm 0.001$ & $0.42 \pm 0.01$ \\
$\tau_{\text {rise+collapse }}$ & $0.020 \pm 0.002$ & $0.10 \pm 0.01$
\end{tabular}



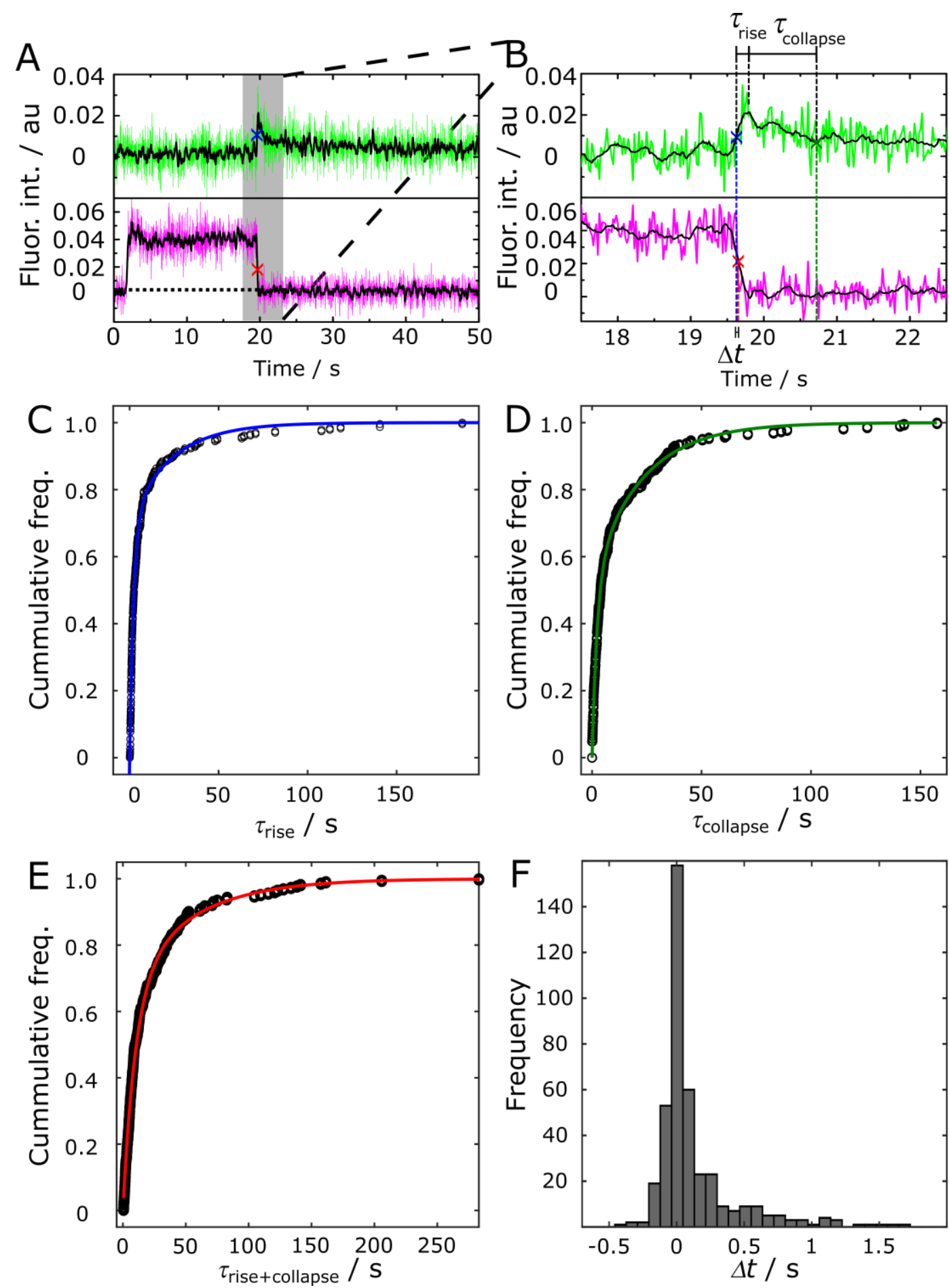

Figure 4.21 (A) Fluorescence intensity time trace of a vesicle (magenta) fusing with the s-PSM (green) with visible lipid mixing with (B) zoom in into the release process and vesicle collapse. Black curves are smoothed data points. The onset of lipid mixing is detected as an increase in s-PSM fluorescence due to diffusion of Atto655 DPPE into the $3 \mathrm{D}$ structure of the vesicle. $\tau_{\text {rise }}$ is defined as the time between the onset of lipid mixing (blue $\mathrm{x}$ ) and the onset of vesicle collapse back into the membrane which is detected as a decrease in fluorescence intensity. $\tau_{\text {collapse }}$ is defined as the time between onset of collapse and the time at which s-PSM fluorescence intensity is constant again (green $\mathrm{x}$ ). $\Delta t$ is defined as the time between lipid mixing (blue $\mathrm{x}$ ) and fusion pore formation (red $\mathrm{x}$ ). (C), (D) Cummulative frequency of $\tau_{\text {rise }}$ and $\tau_{\text {collapse }}(N=234)$. Fitting a bi-exponential equation to the data results in rate constants for $\tau_{\text {rise }}$ of $k_{1}=0.043 \pm 0.004 \mathrm{~s}^{-1}, k_{2}=0.43 \pm 0.01 \mathrm{~s}^{-1}$ and for $\tau_{\text {collapse }}$ of $k_{1}=0.044 \pm 0.001 \mathrm{~s}^{-1}$, $k_{2}=0.42 \pm 0.01 \mathrm{~s}^{1}$. (E) Cummulative frequency of the whole lifetime of the $\Omega$-shape determined by combining $\tau_{\text {rise }}$ and $\tau_{\text {collapse }}(N=234)$. Fitting a bi-exponential equation to the data results rate constants of 
$k_{1}=0.020 \pm 0.002 \mathrm{~s}^{-1}, k_{2}=0.10 \pm 0.01 \mathrm{~s}^{-1}$. (F) Histogram of time differences between lipid mixing and fusion pore formation $(\Delta t, N=455)$. Values lower than -0.7 and higher than $2 \mathrm{~s}$ are not plotted in the histogram $\left(N_{-0.7 s}<\Delta t>2 s=51\right)$.

As described in Chapter 4.4.3, the maximum value to which s-PSM fluorescence increased during the lipid diffusion into the $\Omega$-shape of the vesicle was strongly influenced by the apparent vesicle size with $\rho=0.6022$. To test if vesicle sizes also influenced the different extracted lifetimes, a population analysis was performed. An exemplarily scatter plot of mean vesicle intensity against $\tau_{\text {rise }}$ is depicted in Figure 4.22 and shows no tendencies of larger values of $\tau_{\text {rise }}$ for higher vesicle intensities. This as well as the extracted correlation coefficients summarized in Table 4-4 indicate that all determined lifetimes of the $\Omega$-shape are independent of the vesicle sizes.

Table 4-4 Correlation coefficients of extracted lifetimes of the $\Omega$-shape with the respective mean vesicle fluorescence intensity

\begin{tabular}{l|c} 
& Correlation coefficient \\
\hline$\tau_{\text {rise }}$ & -0.0049 \\
$\tau_{\text {collapse }}$ & 0.0744 \\
$\tau_{\text {rise+collapse }}$ & 0.0344
\end{tabular}

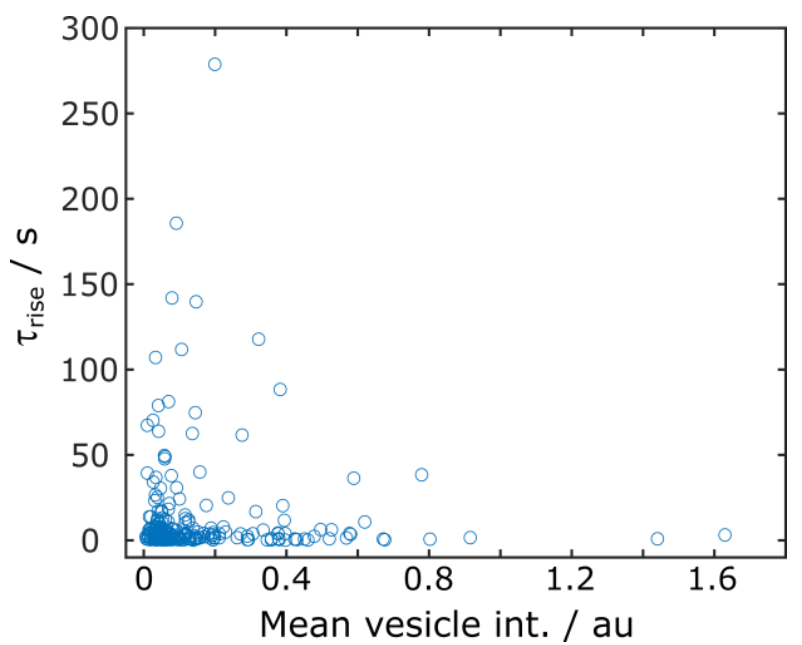

Figure 4.22 Scatter plot of mean vesicle intensities against $\tau_{\text {rise }}$ with a correlation coefficients of $\rho=-0.0049$.

In addition to important time points of the 3D postfusion structure the exact moment of fusion pore formation could be extracted from the SRB fluorescence intensity time trace (Figure 4.21 $\mathrm{B}$, red $\mathrm{x}$ ). Calculating the time difference between the decrease in SRB-fluorescence and increase in s-PSM fluorescence enabled the calculation of the lag time $\Delta t$ between fusion pore formation and lipid diffusion into the $\Omega$-shape. The histogram of $N=455$ extracted values for 
$\Delta t$ is depicted in Figure 4.21 F, where positive values correspond to lipid diffusion into the $\Omega$-shape prior to fusion pore formation. The majority of vesicles show no lag time and the median time difference of $42 \pm 11 \mathrm{~ms}$ suggests that content release and lipid mixing occur almost simultaneously. The error is derived from the resolution limit of $20.83 \mathrm{~ms}$. Besides the exact time point of fusion pore formation in the context of the fusion pathway the release kinetics of SRB were evaluated. As the signal to noise ratios made it impossible to fit individual content release kinetics, peak normalized fluorescence intensity time traces 50 frames prior and after fusion pore formation were extracted from all 840 fusing vesicles and the mean value plotted as a function of time (Figure $4.23 \mathrm{~A}$ ). The black curve is the mean normalized fluorescence intensity with shaded error bars derived from standard deviations and shows that intensity quickly decays starting from $t=1 \mathrm{~s}$. This sharp drop in fluorescence can be associated with the release of SRB into the aqueous space underneath the neighboring f-PSM after the formation of the fusion pore. To extract the mean kinetics of content release of LUVs fusing with the s-PSM a mono-exponential decay function (Equation (4-4)) was fit to the release process (Figure 4.23 B). The mono-exponential fit suggests that content release upon fusion pore formation is a one step process with a rate constant of $k=14.4 \pm 1.4 \mathrm{~s}^{-1}$ and a half-life time calculated as $t_{1 / 2}=\ln (2) \times 1 / k$ of $48 \pm 7 \mathrm{~ms}$. Notably, quantifying the fusion kinetics of e.g. only those vesicles that show full release without visible lipid mixing revealed with $k_{\text {full release }}=12.5 \pm 1.4 \mathrm{~s}^{-1}$ no significant difference.
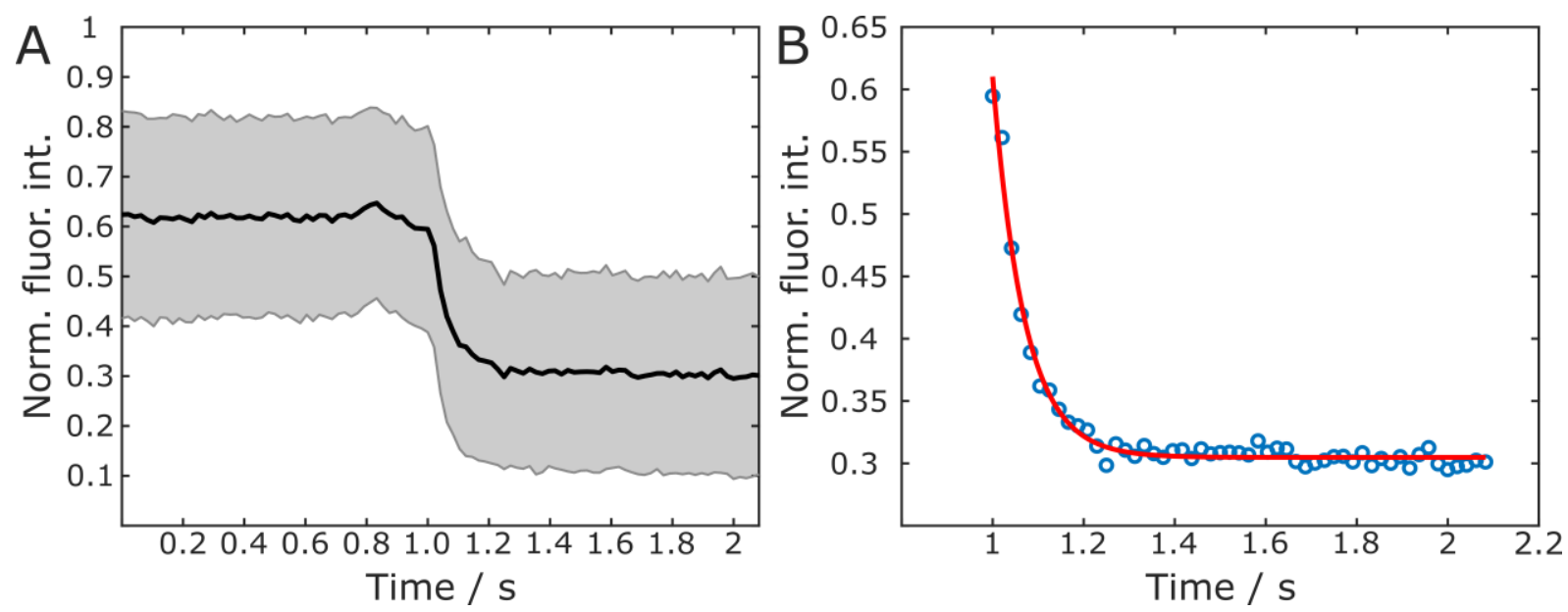

Figure 4.23 (A) Mean normalized content release with SD of all fusing vesicles and (B) mono-exponential decay fit to release process to extract $k=14.4 \pm 1.4 \mathrm{~s}^{-1} \tau=0.069 \pm 0.007 \mathrm{~s}$ and $t_{1 / 2}=0.048 \pm 0.007 \mathrm{~s}$. 


\subsubsection{Docking and fusion of vesicles with the f-PSM}

The vast majority of syb 2 containing large unilamellar vesicles (LUVs) docked immobile to the solid supported part of the $\Delta \mathrm{N} 49$-complex containing pore spanning membrane (s-PSM, open pores, $d=1.2 \mu \mathrm{m})$. However, if vesicles docked to the freestanding part of the PSM (f-PSM) they were found to be fully mobile. To gather information about the mobility of these vesicles and to read out their fluorescence intensity time traces, they were tracked using the ImageJ plugin Mosaic. ${ }^{[103]}$ An exemplarily trajectory of a vesicle docking to and fusing with the f-PSM is depicted in Figure 4.24 A and shows that the vesicle is confined in its movement to one individual f-PSM. This trapped diffusion was observed for all vesicles that dock to the f-PSM leaving only three possible fates: $1^{\text {st }}$ the vesicle remains docked over the whole observation time, $2^{\text {nd }}$ it fuses to the f-PSM, and $3^{\text {rd }}$ it immobilizes at the pore edge. Calculating the current velocity of the vesicle extracted from the trajectory shown in Figure 4.24 A and plotting it as a function of time leads to the velocity map depicted in Figure 4.24 B. The software starts to track the LUV when it is still moving freely inside the 3D solution above the membrane. The calculated velocities prior to docking (black line) are therefore only rough estimates. However, it is clear that the vesicle significantly slows down once it docks to the f-PSM, possibly due to interaction of the respective SNARE-binding domains. It then has a constant velocity of $\sim 10 \mu \mathrm{m} \mathrm{s}^{-1}$ over the whole observation time $\left(D=0.13 \mu \mathrm{m}^{2} \mathrm{~s}^{-1}\right)$. The trajectory was further used to place a mobile ROI on the vesicle, fluorescence intensity was read out, and plotted as a function of time (Figure $4.24 \mathrm{C}$ ). As the vesicle is tracked before it docks to the f-PSM no increase in fluorescence is recorded upon docking. Full content release upon fusion pore formation of the vesicle after $\sim 89 \mathrm{~s}$ can be detected as a sharp decrease in fluorescence intensity to baseline level. Notably, no information about the diffusion of lipids from the f-PSM into the vesicle membrane could be extracted due to the higher baseline level of lipid fluorescence on the unquenched f-PSM. 

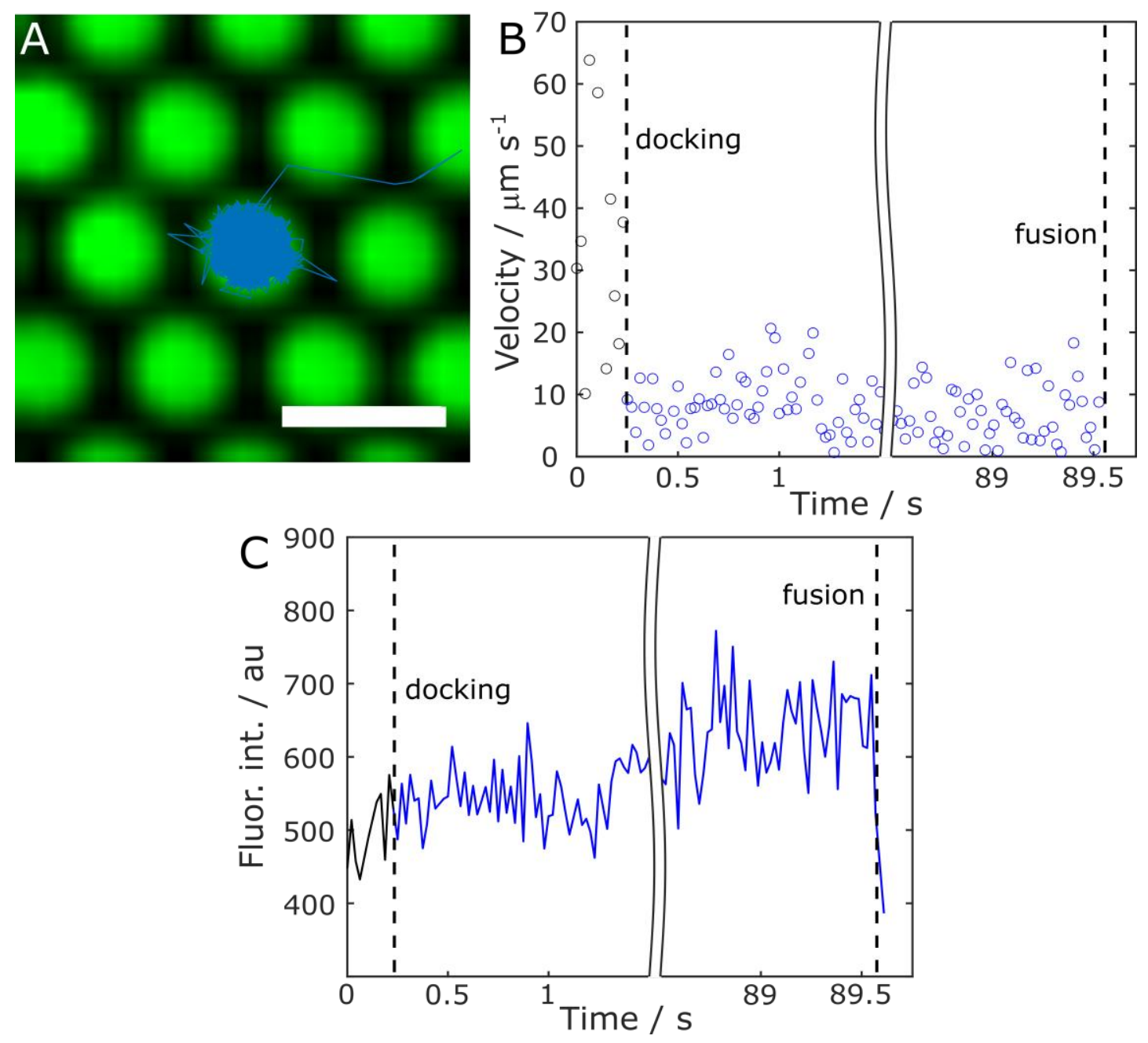

Figure 4.24 Exemplarily event of a vesicle fusing with the f-PSM. (A) Fluorescence micrograph of the PSM (green) and the extracted trajectory of the vesicle (magenta). Scale bar $=2 \mu \mathrm{m}$. (B) Velocity map of the vesicle in the pre-docked state (black) and of the vesicle docked to the f-PSM (blue). The vesicle slows down until it docks to the f-PSM with a mean diffusion coefficient of $D=0.13 \mu \mathrm{m}^{2} \mathrm{~s}^{-1}$. Due to the long docking time, data between 1.5 and $88.5 \mathrm{~s}$ are not displayed in the graph. (C) Respective fluorescence intensity time trace of vesicle fluorescence. Upon fusion pore formation and full content release, fluorescence intensity drops after $\sim 89 \mathrm{~s}$.

As trajectories were split in parts were the vesicle diffused inside the f-PSM and in the bulk solution, the trapped diffusion inside the f-PSM was visible as a plateau in the MSD-plot for longer time scales shown in Figure 4.25 A. Notably, for short time intervals of at least $200 \mathrm{~ms}$ these vesicles showed unhindered diffusion. The diffusion coefficient of $N=14$ vesicles could thus be extracted from the slope $(m)$ of this part of the MSD-plot (Figure 4.25 A, B, blue line) as $m=4 D$. Determined diffusion coefficients of docked vesicles ranged from 0.03 to $0.5 \mu \mathrm{m}^{2} \mathrm{~s}^{-1}$ (Figure 4.25 C) with a mean value of $0.15 \pm 0.15 \mu \mathrm{m}^{2} \mathrm{~s}^{-1}$. 

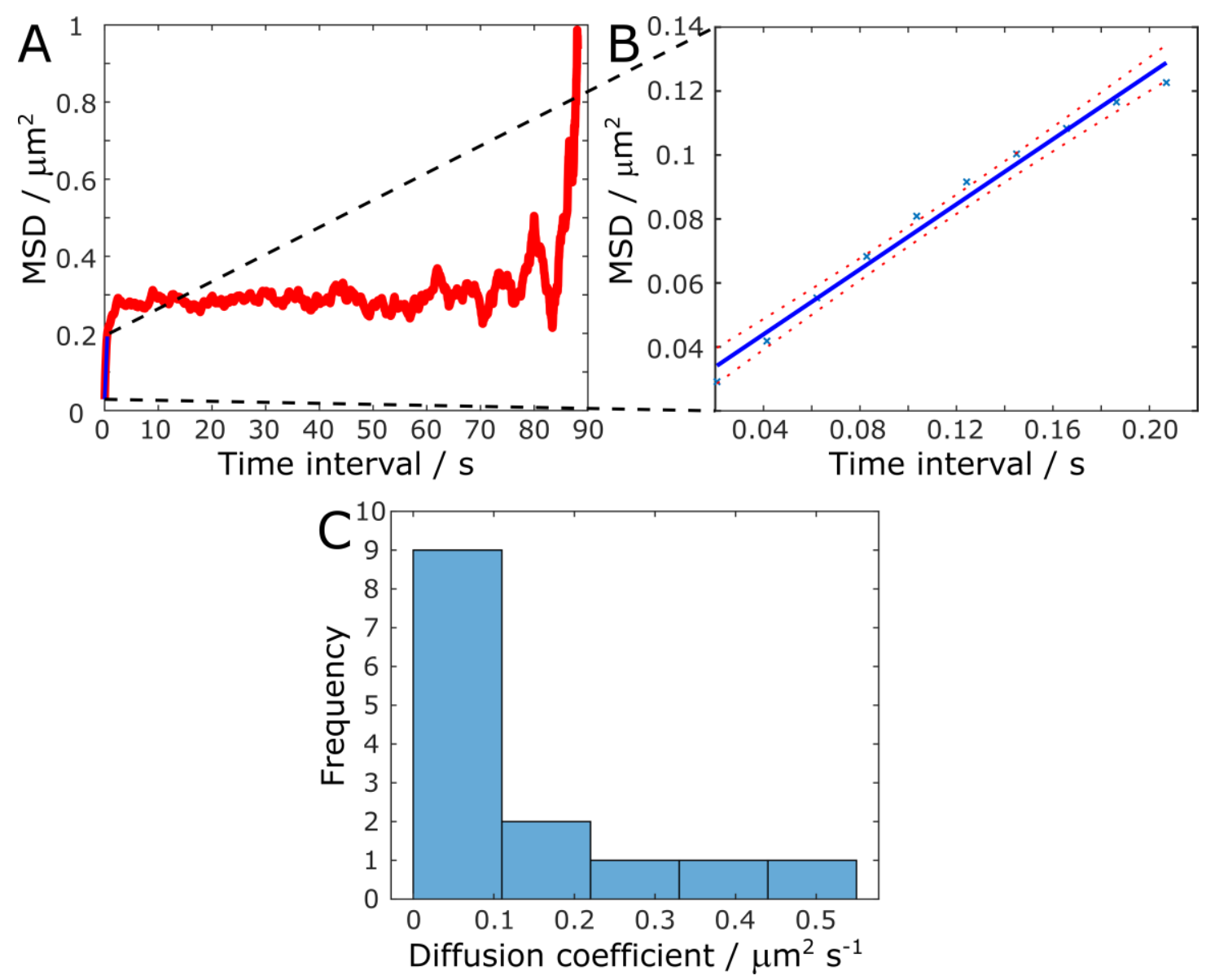

Figure 4.25 (A) Exemplarily mean squared displacement (MSD) extracted from the trajectory shown in Figure 4.24. The vesicle shows confined diffusion over 80 seconds, indicated by the constant value of the MSD of $0.3 \mu \mathrm{m}^{2}$ until it ultimately fuses with the f-PSM. (B) At small time intervals of several hundreds of milliseconds the vesicle diffuses freely indicated by a constant slope of the MSD. The diffusion coefficient $D$ was extracted as the slope (blue line, $4 D$ ) of this part of the MSD plot. (C) Histogram of diffusion coefficients of $N=14$ vesicles that fused with the f-PSM or docked to the f-PSM over the whole observation time.

\subsubsection{Content release into closed cavities}

Single-vesicle content release events of SRB-filled LUVs with PSMs formed on a porous substrate with open pores were used to identify and quantify different fusion pathways as well as kinetics of the fusion process as described in Chapter 4.4.1-4.4.6. Content transfer across the target membrane could be directly visualized as an increase in fluorescence inside the aqueous space underneath the f-PSM following SNARE-mediated fusion pore formation (Figure 4.10). However, due to the vary rapid release kinetics and diffusion of SRB out of the open pore and into the solution underneath the porous substrate, these events could only be observed occasionally. A more elegant way to better visualize the process of content release would be the use of porous substrates with closed compartment that can uptake the released content and still retain the advantages of PSMs compared to SLBs. To achieve this, PSMs were formed by spreading GUVs with reconstituted $\Delta \mathrm{N} 49$-complex 
(DOPC/POPE/POPS/cholesterol/Atto488 DPPE; 5/1.99/1/2/0.01 (n/n), nominal p/1 1:500) as described in Chapter 3.2.5 on porous substrates with a pore diameter of $3.5 \mu \mathrm{m}$ and a pore depth of $250 \mathrm{~nm}$. An exemplarily fluorescence micrograph of a membrane patch used to perform single-vesicle content release experiments is shown in Figure 4.26. Single-vesicle fusion experiments were performed as described in Chapter 3.3.4 with slight modifications. For the first 1200 frames after LUV addition both membrane and vesicle fluorescence intensity were recorded by dynamically switching between the two channels. Then, single-vesicle fusion events were recorded in single channel mode by only detecting SRB-fluorescence with $13.04 \mathrm{~ms}$ per frame over 30000 frames.

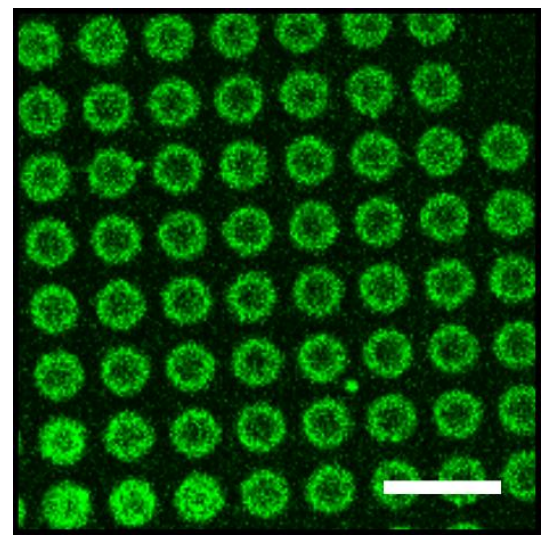

Figure 4.26 Fluorescence micrograph of a Atto488 DPPE labeled PSMs (green) with reconstituted $\triangle$ N49-complex (DOPC/POPE/POPS/cholesterol/Atto488 DPPE; 5/1.99/1/2/0.01 (n/n), nominal p/l 1:500). The PSM was formed on a porous substrate with closed cavities with a depth of $250 \mathrm{~nm}$ and a pore dimeter of $3.5 \mu \mathrm{m}$. Scale bar $=10 \mu \mathrm{m}$.

A representative fluorescence intensity time lapse of a fusion event is depicted in Figure 4.27 A with vesicle fluorescence (false colored in magenta) recorded as a function of time. Target membrane fluorescence was recorded in the first frames of the time series and is used as an overlay to determine the location of vesicle docking and the direction of content release. Figure 4.27 C shows the fluorescence intensity time trace of SRB read out from a $4 x 4$ pixel ROI placed on top of the docked vesicle (1, black) and the aqueous space underneath the f-PSM (2, magenta). Vesicle docking to the edge of the f-PSM leads to an increase in fluorescence intensity of ROI 1 (I) while no change is detected inside ROI 2. After fusion pore formation vesicle content is released into the cavity underneath the f-PSM (II) resulting in a decrease in vesicular intensity concomitant with an increase in fluorescence intensity inside the $2^{\text {nd }}$ aqueous compartment. Due to the shallow depth of the cavity and the thin aqueous layer between s-PSM and solid support, SRB slowly diffuses out of the cavity and into the neighboring compartments. In other cases, vesicular fluorescence intensity exhibits a peak upon fusion pore formation (Figure 4.27 B, D). This increase is a result of dequenching of concentration quenched SRB 
upon fusion pore formation and indicates a hindered content release from the vesicle. Vesicle bursting, as it is the origin for such a peak for vesicles fusing with PSMs formed on open pores, can be ruled out since it would not lead to the content to be transferred into the cavity. A hindered release which leads to a dequenching of SRB fluorescence can be caused by several factors. Firstly, the shallow cavity next to the docking site of the vesicle could be too small to uptake the whole interior solution if the vesicle is too large. To correlate vesicle sizes with an existing peak, the peak was defined as the difference between maximum fluorescence of the vesicle and its mean intensity value. Calculating the correlation coefficient between this value and the mean fluorescence of the vesicle leads to $\rho=0.3836$. Thus, vesicles sizes seem to have an influence on the existence as well as height of the peak which indicates that the dequenching effect is stronger for larger vesicles. In addition, while being docked close to the edge of the pore not necessarily leads to a peak in the intensity time trace (e.g. Figure 4.27 A, C) it seems to be essential for a peak to appear in the first place. Vesicles docked relatively far away from the cavities on s-PSM released their content into different cavities simultaneously without a visible peak.
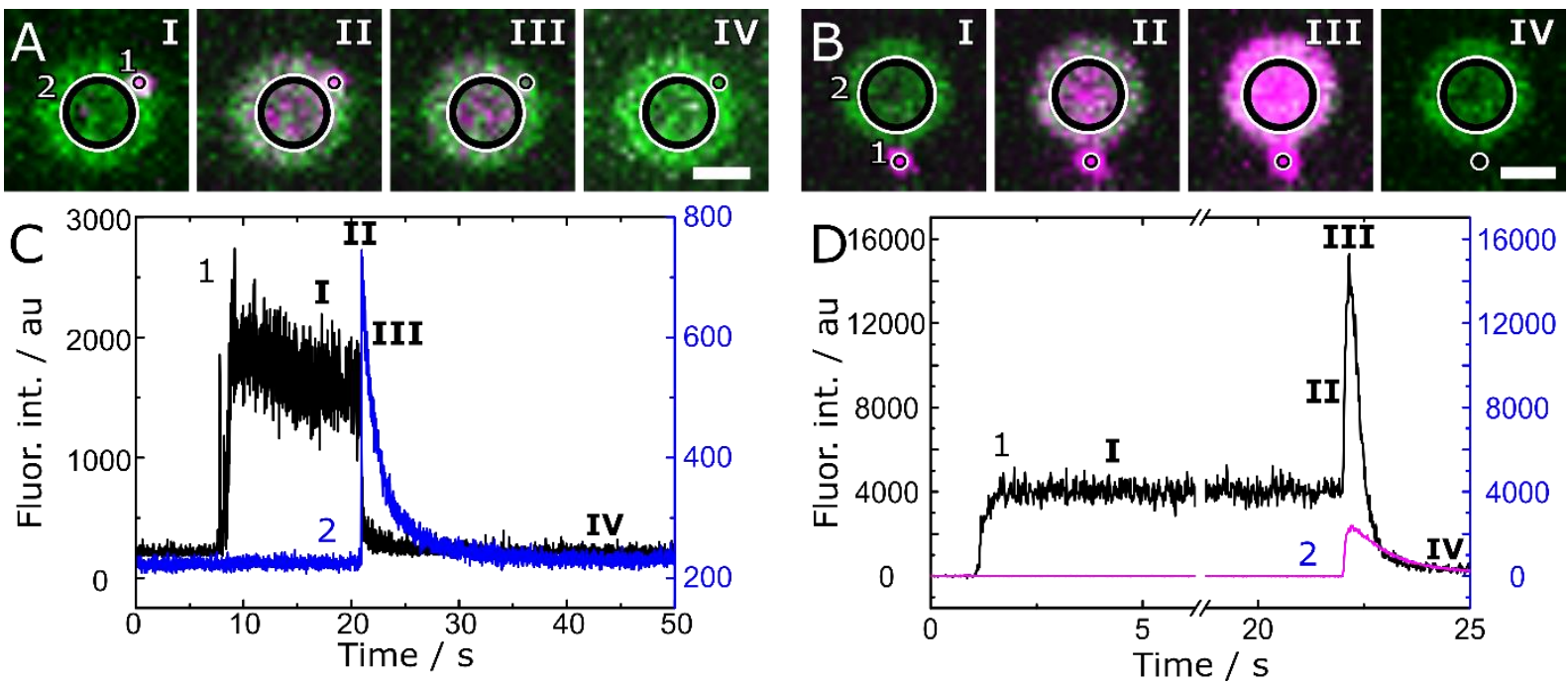

Figure 4.27 Representative single-vesicle content release events using porous substrates with closed compartments. (A) and (B) Time lapse fluorescence images of a SRB-filled syb 2 LUV (false colored in magenta) docked to the Atto488 DPPE doped $\triangle \mathrm{N} 49$ containing s-PSM (green). ROI 1 and 2 were used to read out fluorescence intensity of the vesicle (1) as well as the cavity underneath the f-PSM (2) as a function of time (C, D). Scale bars $=2 \mu \mathrm{m}$. Upon fusion pore formation, vesicle content is released through the fusion pore into the $2^{\text {nd }}$ aqueous compartment underneath the neighboring f-PSM (II-III) resulting in an increase in intensity (ROI 2). The content then slowly diffuses out of the cavity (III-IV). Content release can either be monitored as a direct decrease of vesicular intensity (C) or a first increase due to dequenching of the concentration quenched fluorophore followed by a decrease (D).

In some cases the peak in fluorescence intensity is accompanied by a very slow release kinetic (Figure $4.27 \mathrm{D}$ ). To quantify this effect, the mean normalized release profile of $N=67$ fusion 
events was extracted from the raw data (Figure 4.28 A). The peak in fluorescence upon fusion pore formation described in Figure 4.27 D is clearly visible in the averaged data set. Only a bi-exponential fit (Equation (4-8)) to the data was possible, suggesting either two subpopulations in the data or that the release process is dominated by two different kinetics (Figure $4.28 \mathrm{~B}$ ). The fit resulted in a short half-life time of $t_{1 / 2,1}=0.021 \pm 0.002 \mathrm{~s}$ and a long half-life time of $t_{1 / 2,2}=1.5 \pm 0.1 \mathrm{~s}$.

$$
y(x)=a \cdot \exp \left(-k_{1} x\right)+b \cdot \exp \left(-k_{2} x\right)
$$
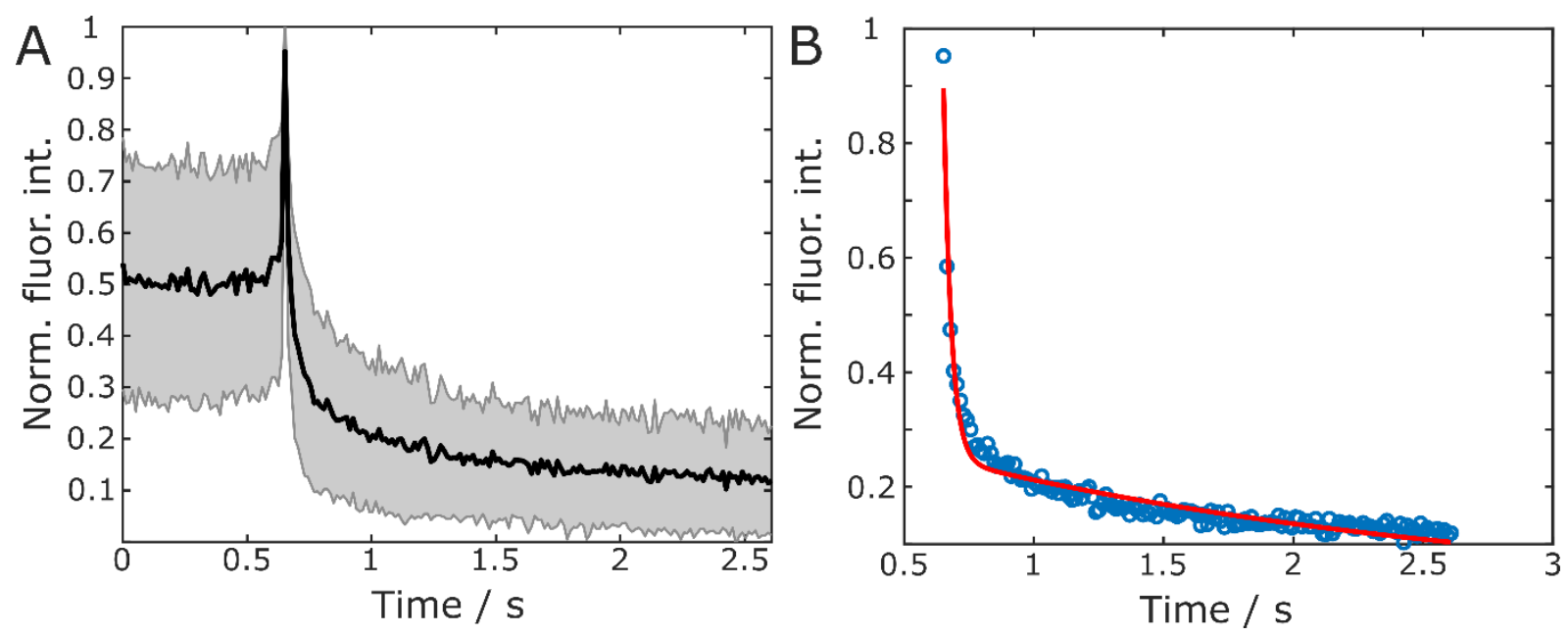

Figure 4.28 (A) Mean normalized content release with SD of all fusing vesicles (gray shaded area, $N=67$ ). The release profile exhibits a sharp increase upon fusion pore formation followed by decrease of fluorescence to baseline level. (B) A bi-exponential decay function (red line) was fit to the release profile to extract $k_{1}=33.7 \pm 3.3 \mathrm{~s}^{-1}$ and $k_{2}=0.45 \pm 0.04 \mathrm{~s}^{-1}$. This results in half-life times of the release process of $t_{1 / 2,1}=0.021 \pm 0.002 \mathrm{~s}$ and $t_{1 / 2,2}=1.5 \pm 0.1 \mathrm{~s}$.

Due to the high signal to noise ratio of individual fluorescence intensity time traces of single fluorescence recordings, it was possible to evaluate the release kinetics of each individual single-vesicle fusion event. The individual release profiles were well described with a mono-exponential decay function (Figure 4.29 A, B) suggesting a process dominated by one rate limiting step. However, the release can either be very fast (Figure 4.29 A) with an exemplarily time constant of $k=24.9 \pm 1.5 \mathrm{~s}^{-1}$ or slow (Figure 4.29 B) with $k=4.6 \pm 0.1 \mathrm{~s}^{-1}$. The distribution of calculated half-life times of $N=67$ individual release kinetics is shown in Figure 4.29 C and exhibits a large population of fast release kinetics and a tail to longer half-life times. Calculating the correlation coefficient between mean vesicle intensity and half-life time of the release process leads to $\rho=0.0981$. This indicates that vesicle size has little to no influence on the observed differences in kinetics of content release. Also the position of the docked vesicle has no influence on the kinetics of the release process, as both slow and fast 
kinetics are observed for vesicles docked close or far away from the $2^{\text {nd }}$ aqueous compartment. One common factor of events with slow release $\left(t_{1 / 2}>100 \mathrm{~ms}\right)$ is a peak in the fluorescence intensity time trace. However, this peak can also be seen in vesicles with fast release kinetics of $t_{1 / 2}<100 \mathrm{~ms}$ thus it is not clear what causes outliers of slow content release. The majority of release events $(84 \%)$ show a short half-life time of the release profile of $t_{1 / 2}<100 \mathrm{~ms}$.

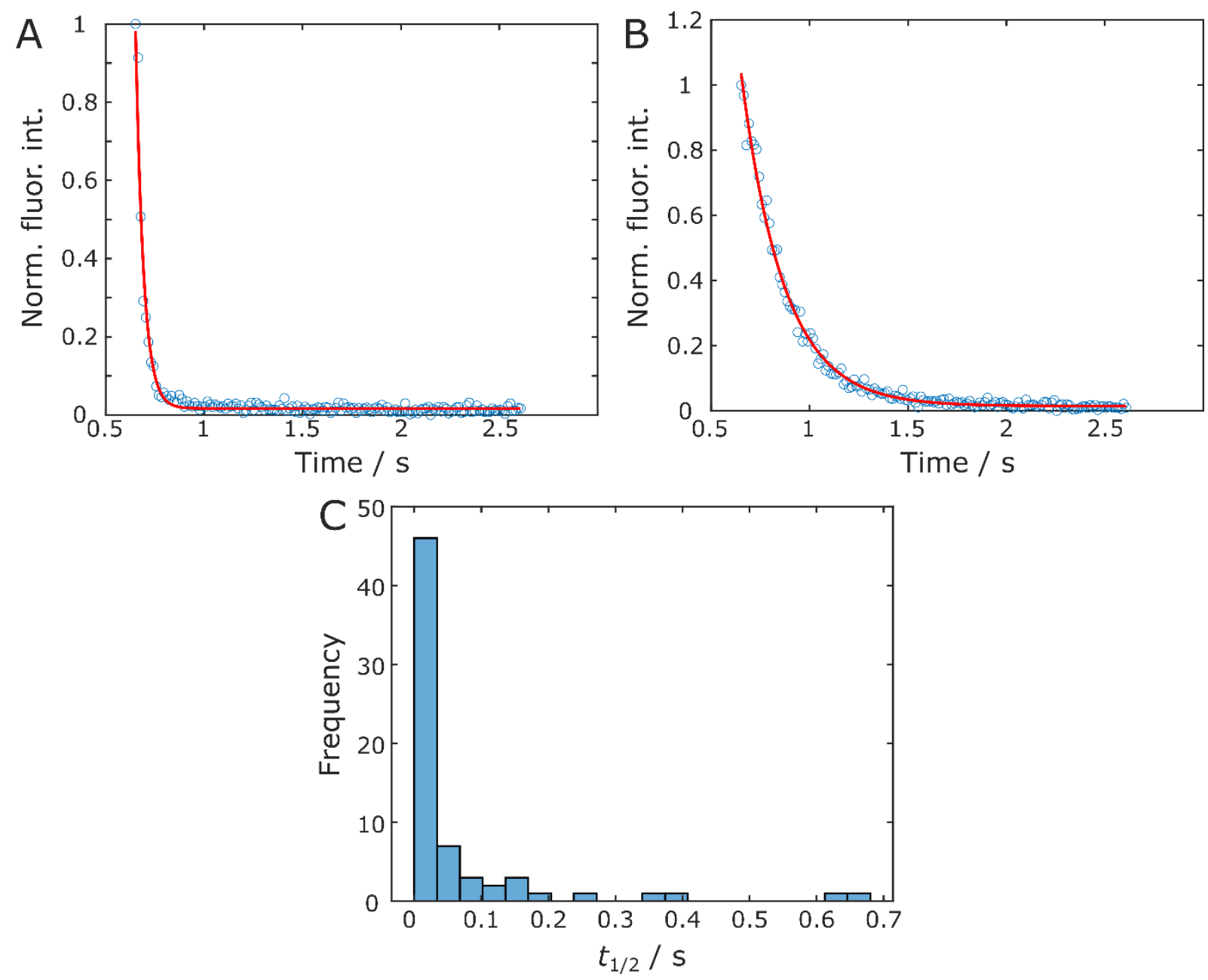

Figure 4.29 Evaluation of $N=67$ individual release profiles. (A) Mono-exponential decay fit to the release profile of an exemplarily fast content release leads to a rate constant of $k=24.9 \pm 1.5 \mathrm{~s}^{-1}$. (A) Mono-exponential decay fit to the release profile of an exemplarily slow content release leads to a rate constant of $k=4.6 \pm 0.1 \mathrm{~s}^{-1}$. (C) Histogram of content release half-life times by fitting a mono-exponential decay to $N=67$ release profiles. 


\section{Discussion}

The goal of this thesis was to establish the simultaneous detection of content release and lipid mixing in a SNARE-mediated single-vesicle fusion assay on pore-spanning membranes (PSMs) to gain new insights in the process of fusion pore formation and possible fusion pathways. To achieve this, sulforhodamine B (SRB) was encapsulated into syb 2 doped large unilamellar vesicles and individual fusion events with $\Delta$ N49-complex containing PSMs investigated by means of two color spinning disc microscopy with $20.83 \mathrm{~ms}$ time resolution. This enabled the direct observation of fusion pore formation, it's interplay with lipid diffusion into the vesicle, and the quantification of both content release kinetics as well as different fusion pathways. ${ }^{[95]}$

\subsection{Reconstitution efficiency of SNAREs into GUVs}

The t-SNARE acceptor complex $\triangle \mathrm{N} 49$ and v-SNARE synaptobrevin 2 (syb 2) were functionally reconstituted into giant and large unilamellar vesicles (GUVs, LUVs), respectively. Proteins were inserted into lipid bilayers composed of DOPC/POPE/POPS/cholesterol; 5/2/1/2 (n/n) thus mimicking the composition in the plasma membrane of synapses and that of synaptic vesicles ${ }^{[88-90]}$ at a nominal protein to lipid ratio of 1:500 as described by Schwenen et al. with slight modifications. ${ }^{[85]}$ As a starting material for LUV and GUV formation, proteo-SUVs were formed from mixed micelles of lipids, proteins, and detergent followed by the removal of the latter through rapid dilution below the critical micelle concentration and size exclusion chromatography done twice. This method has proven to lead to the formation of fusogenic proteo-SUVs with a reconstitution efficiency of $\sim 100 \%{ }^{\left[{ }^{[5,86]}\right.}$ Syb 2 doped LUVs with a mean diameter of $240 \mathrm{~nm}$ used for single-vesicle experiments were shown to have an effective protein to lipid ratio of 1:600 quantified by density gradient and SDS-PAGE analysis. ${ }^{[86]}$ Assuming no preferred direction of insertion during reconstitution, this reflects $\sim 930$ syb 2 molecules/LUV or $\sim 2500$ molecules per $\mu \mathrm{m}^{2}$ facing towards the outer solution. While this value is smaller than the estimated 12000 copies of syb 2 per $\mu \mathrm{m}^{2}$ found in synaptic vesicles, ${ }^{[90]}$ it was proven to be sufficient to drive efficient membrane fusion. ${ }^{[85,115]}$ In this work, the reconstitution efficiency of $\Delta \mathrm{N} 49$ into GUVs was determined in a quantitative fluorescence study of a labeled protein construct and turned out to be $26 \pm 24 \%$ which results in an effective p/l ratio of 1:1900 (see Chapter 4.2). ${ }^{[95]}$ While this number is very high compared to the estimated $<1 \%$ determined by Kuhlmann, it is very low in respect to the quantitative reconstitution of $100 \%$ determined by Witkowska et al. ${ }^{[61,115]}$ In both studies the 
$\triangle$ N49-complex was reconstituted into SUVs and from those, GUVs produced via electroformation on indium tin oxide (ITO) coated glass surfaces. The high reconstitution efficiency reported by Witkowska et al. can be explained due to the use of natural brain lipid extracts or differences in the labeling procedure of the acceptor complex compared to this work. Here, the acceptor complex was fluorescently labeled at the small syb 2 fragment thus ensuring that only the fusion active $\Delta \mathrm{N} 49$-complex is visualized in GUVs. However, in their study the fluorescent label was attached to SNAP25a and it is likely that also the unfusogenic syntaxin/SNAP25a 2:1 complex was accounted for in the reconstitution efficiency. Notably, the reported reconstitution efficiency did not change when ITO coated glass surfaces were exchanged by platinum wires to perform the electroformation. ${ }^{[16]}$ Kuhlmann on the other hand, who had difficulties visualizing the labeled t-SNARE complex inside GUVs, had to increase the nominal $\mathrm{p} / 1$ ratio to $1: 125$ and could only estimate and not quantify the reconstitution efficiency. The only difference between the two protocols is a gentler electroformation procedure used in the present work that involved a slow increase in applied voltage which could cause the change in reconstitution efficiency. It is clear that the final step of electroformation is the main cause for the loss of protein in both studies, as reconstitution into SUVs proved to be quantitative. ${ }^{[86,117,118]}$ In agreement with Kuhlmann large aggregates of lipids with high concentration of proteins were visible inside the obtained GUV-solution that are most likely formed during the drying process of proteo-SUVs prior to electroformation. This drying process is known to cause loss in protein functionality due to denaturation of proteins. ${ }^{[119,120]}$ One possible way to stabilize the protein during drying would be the addition of sucrose to the proteo-SUV solution which lead to the recovery of protein activity. ${ }^{[120]}$ While disaccharides prevent protein denaturation in the absence of water by forming hydrogen bonds with the polar head groups of the protein in a process called preferential exclusion, ${ }^{[121]}$ high concentration of approx. $>0.86 \mathrm{~g}$ sucrose/g lipid inhibit the fusion of proteo-SUVs on the ITO surface and subsequently the formation of GUVs. ${ }^{[120,122]}$ Even thought this effect is diminished at lower sucrose concentrations it is unclear whether it would still influence the fusion behavior of single vesicles to PSMs formed from these GUVs. Hence, an alternative method for the GUV formation could be useful to achieve higher reconstitution efficiencies. Protocols that are based on direct insertion of proteins into preformed GUVs might lead to high reconstitution efficiencies with proteins preferably facing with their cytosolic domain into the bulk solution. ${ }^{[123,124]}$ While this is usually advantageous for studies with proteo-GUVs, the spreading process to produce pore-spanning membranes (PSMs) used in this work results in the outer membrane of the GUV facing towards the porous substrates. As vesicles are added from above 
to the PSM the SNARE-binding motifs of the acceptor complex would not be accessible for the single-vesicle fusion experiments. Other methods based on fusion of LUVs to preformed GUVS $^{[125-127]}$ or droplet transfer ${ }^{[128,129]}$ would either lead to low amounts of protein densities or too small vesicle diameters. A protocol that both avoids drying of the protein as well as produces unilamellar GUVs with large diameters and correct protein orientation is based on the use of microfluidic flow chambers for the formation of droplet stabilized GUVs (dsGUVs). ${ }^{[130]}$ However, it has to be tested if the surfactants used for the formation of dsGUVs are completely excluded from the lipid bilayer when releasing the GUVs in the last preparation step. This is important, as changes in the mechanical properties of the target membrane can influence the fusion efficiency on a single-vesicle level. ${ }^{[131]}$

In summary, the effective protein to lipid ratio of the t-SNARE acceptor complex $\Delta$ N49 inside GUVs was determined to be $1: 1900$ with a mean diameter of $d_{\mathrm{GUV}}=15 \mu \mathrm{m}$. Assuming a mean lipid area of $0.65 \mathrm{~nm}^{2}$ this would lead to $\sim 800$ proteins per $\mu \mathrm{m}^{2}$ PSM accessible for the interaction with syb 2 doped LUVs with large variations in protein densities between individual membrane patches. ${ }^{[107]}$ Considering an estimated syx 1 density in rat chromaffin cells of 540 to $\sim 1800$ molecules per $\mu \mathrm{m}^{2}$ the amount of accessible acceptor complex found in this work should be sufficient to drive membrane fusion. ${ }^{[132,133]}$

\subsection{Mobility of proteins and lipids in model membrane systems}

The process of synaptic vesicle exocytosis requires the lateral mobility of syx 1A and SNAP25 inside the presynaptic membrane, partly as they dynamically form the t-SNARE acceptor complex that is necessary for the interaction with syb $2 \cdot{ }^{[108]}$ In vivo syx $1 \mathrm{~A}$ is anchored inside the membrane via a $C$-terminal transmembrane domain (TMD) and SNAP25 covalently bound to the plasma membrane via a palmitoylated linker connecting the two SNARE motifs. In this work the fusion active $\Delta \mathrm{N} 49$ acceptor complex is solely anchored inside the target membrane via the syx 1A TMD. To better understand the diffusive behavior of the full acceptor complex inside the target PSMs the diffusion coefficients of lipids and $\Delta$ N49-Atto488 were quantified inside the solid supported part of the PSM (s-PSM). To investigate the influence of the underlying substrate on protein mobility, the diffusion coefficients were additionally determined inside the membrane of GUVs using the same vesicle populations. The mobility of $\triangle \mathrm{N} 49-$ Atto488 and Atto390 DPPE inside GUVs was investigated by performing fluorescence recovery after photobleaching (FRAP) experiments on the top plane of the GUV (Chapter 4.3.1). The data was evaluated according to Soumpasis and Kang et al. and lead to a diffusion 
coefficient of $D_{\text {Atto390 DPPE }}=9 \pm 3 \mu \mathrm{m}^{2} \mathrm{~s}^{-1}$ and $D_{\Delta \mathrm{N} 49}=5 \pm 3 \mu \mathrm{m}^{2} \mathrm{~s}^{-1}$ with a mobile fraction of each $100 \%$ (Table 5-1). ${ }^{[97,98]}$ This Protein mobility is similar to the one of syx 1A determined by Bacia et al. (Table 5-1)). ${ }^{[134]}$ Values, however, vary significantly compared to the diffusion coefficients inside GUVs of $D_{\mathrm{PE}}=2.7 \mu \mathrm{m}^{2} \mathrm{~s}^{-1}$ and $D_{\Delta \mathrm{N} 49}=1.2 \mu \mathrm{m}^{2} \mathrm{~s}^{-1}$ determined by Witkowska et al. ${ }^{[115]}$ While they used very similar lipid mixtures and the same $\mathrm{p} / \mathrm{l}$ ratio as in the present work, a more simplified evaluation procedure was applied that does not take into account the diffusion of molecules into the bleach spot during the process of photobleaching. However, as shown by Kang et al. obtained diffusion coefficients can be underestimated up to several orders of magnitude when ignoring the potential difference between nominal and effective bleach radii. ${ }^{[98]}$ Indeed, when only using the nominal bleach radius, very similar diffusion coefficients compared to Witkowska et al. with $D_{\text {Atto390 DPPE }}=4 \pm 1 \mu \mathrm{m}^{2} \mathrm{~s}^{-1}$ and $D_{\Delta \mathrm{N} 49}=2 \pm 1 \mu \mathrm{m}^{2} \mathrm{~s}^{-1}$ were found. The inclusion of the effective bleach radius also enables a better comparability with data obtained by fluorescence correlation spectroscopy (FCS) which usually lead to diffusion coefficients that are larger by a factor of 2-3. ${ }^{[135]}$ Thus the obtained diffusion coefficient of Atto390 DPPE is in good agreement with FCS measurements that, depending on the used lipid mixtures, result in diffusion coefficients between 6 and $12 \mu \mathrm{m}^{2} \mathrm{~s}^{-1} \cdot{ }^{[135-138]}$ FCS was also used previously to investigate the diffusive properties of lipids and proteins in the freestanding part of PSMs (f-PSMs) with obtained values of $D_{\text {DPPE }}($ f-PSM $)=7.7 \pm 0.4 \mu \mathrm{m}^{2} \mathrm{~s}^{-1}, \quad D_{\text {Syx-TMD }}(\mathrm{f}-\mathrm{PSM})=3.4 \pm 0.2 \mu \mathrm{m}^{2} \mathrm{~s}^{-1}, \quad$ and $D_{\text {syx 1A }}(\mathrm{f}-\mathrm{PSM})=2.3 \pm 0.5 \mu \mathrm{m}^{2} \mathrm{~s}^{-1} \cdot{ }^{[85]}$ Diffusion of molecules inside the f-PSM is very similar to the freely suspended membrane of GUVs highlighting its suitability as a planar model membrane with unhindered diffusing components that is easily accessible via fluorescence microscopy. ${ }^{[134]}$ In comparison to this, the diffusion of the pure syx 1A TMD inside the solid supported part of the PSM was previously determined by means of indirect FRAP experiments in combination with finite element simulations to be $D_{\text {Syx-TMD }}$ (s-PSM) $=1.0 \pm 0.5 \mu \mathrm{m}^{2} \mathrm{~s}^{-1}$, suggesting that the membrane-surface interaction induces reduced protein mobility. ${ }^{[86]}$ As the full acceptor complex contains a large cytosolic domain and hence might interact more strongly with the pore rim, the same approach was used to quantify its diffusion coefficient inside the s-PSM. Fluorescence intensity of $\triangle \mathrm{N} 49$-Atto488 was fully bleached inside one f-PSM so that the observed recovery is mainly dominated by the diffusion of molecules from the surrounding pore rim. To account for the faster diffusing of fluorophores once they enter the bleached f-PSM, the obtained data was compared with simulated recovery curves that used a fixed value for the diffusion coefficient inside the f-PSM while varying the coefficient of the s-PSM (see 
Chapter 4.3.2). Thus, diffusion coefficients of $D_{\mathrm{DPPE}}(\mathrm{s}-\mathrm{PSM})=2 \pm 1 \mu \mathrm{m}^{2} \mathrm{~s}^{-1}$ and $D_{\Delta \mathrm{N} 49}(\mathrm{~s}-\mathrm{PSM})=1.0 \pm 0.5 \mu \mathrm{m}^{2} \mathrm{~s}^{-1}$ were obtained showing no difference compared to the pure syx 1A-TMD. ${ }^{[95]}$

While lipids showed a mobile fraction of $100 \%$ during the recovery time of $6 \mathrm{~s}$, a mobile fraction of the acceptor complex of $70 \pm 21 \%$ in the time scale of $20 \mathrm{~s}$ was determined. This number is only an estimate and restricted to the observation time as only mobile proteins are detected during indirect FRAP experiments. It is possible that proteins facing with the cytosolic domain towards the solid support are completely immobilized. However, the fact that a significant amount of proteins can diffuse freely inside the s-PSM is mainly caused by the self-assembled monolayer (SAM) on the pore rim leading to a larger space between membrane and solid support. Ramakrishnan et al. formed PSMs containing the t-SNAREs syx 1A and SNAP25 on silicon dioxide functionalized porous substrates and found a t-SNARE complex that is fully immobile on the pore rim. ${ }^{[139]}$ This high immobile fraction of syntaxin $1 \mathrm{~A}$ inside lipid bilayers that are in direct contact with the surface was also found in pure supported lipid bilayers (SLB) formed on quartz. ${ }^{[72]}$ Uncoupling the SLB from the solid support, similar to the introduction of a SAM, by either introducing poly ethylene glycol (PEG)-spacers or spreading proteo-SUVs on a preformed monolayer produced via Langmuir Blodgett transfer results in increased mobile fractions of Syx $1 \mathrm{~A}$ to $68-83 \% .^{[78]}$

Interaction of the membrane with the solid support also influences the diffusion coefficients of lipids and reduces their mobility by a factor of 4-5 compared to GUVs. For SLBs produced by directly spreading SUVs on solid supports, diffusion coefficients are even more reduced. ${ }^{[72,78,140]}$ While only the lower leaflet directly interacts with the solid support, high frictional coupling of both monolayers leads to equal diffusion coefficients of the two leaflets for solid supported membranes. ${ }^{[140-142]}$ Notably, diffusion coefficients of lipids inside the s-PSM are in the same range of those obtained for inverted plasma membranes sheets $\left(D_{\mathrm{PC}}=0.5 \mu \mathrm{m}^{2} \mathrm{~s}^{-1}\right){ }^{[143]}$

When comparing the diffusion coefficients of lipids and proteins it turns out that protein diffusion is usually by a factor of 2-3 times lower compared to lipids in GUVs, s-PSMs, and f-PSMs. This is in agreement with other studies performed on GUVs and solid supported membranes. ${ }^{[15,120]}$ While a larger hydrodynamic radius of the protein has a minor impact on the diffusion coefficient based on the Saffmann Delbrück model, the main different is caused by the TMD of syntaxin spanning both the upper and lower leaflet. ${ }^{[138,144]}$ 
Additionally, syntaxin-1A-GFP was found to be fully mobile in rat spinal cord neurons with a mean diffusion coefficient at synapses of $0.07 \mu^{2} \mathrm{~s}^{-1} \cdot{ }^{[145]}$ While being fully mobile the smaller diffusion coefficient can be caused by the high protein density inside synapses and clustering of syx 1A with receptor lipids and proteins inside the presynaptic membrane. ${ }^{[78,146,147]}$

In summary, FRAP experiments on GUVs with an improved evaluation procedure, as described previously, were used to obtain diffusion coefficients of $D_{\mathrm{DPPE}}=9 \pm 3 \mu \mathrm{m}^{2} \mathrm{~s}^{-1}$ and $D_{\Delta \mathrm{N} 49}=5 \pm 3 \mu \mathrm{m}^{2} \mathrm{~s}^{-1}$ that are in good agreement with values obtained by means of FCS measurements. ${ }^{[134,137]}$ Performing indirect FRAP experiments to extract the diffusion coefficients of the two molecules inside the s-PSM with the same GUV populations enabled the direct comparison between the two model membrane systems. Lipid and protein mobility was reduced by a factor of 4-5 to $D_{\text {DPPE }}(\mathrm{s}-\mathrm{PSM})=2 \pm 1 \mu \mathrm{m}^{2} \mathrm{~s}^{-1}$ and $D_{\Delta \mathrm{N} 49}(\mathrm{~s}-\mathrm{PSM})=1.0 \pm 0.5 \mu \mathrm{m}^{2} \mathrm{~s}^{-1}$ due to membrane substrate interactions. However, as proteins retain large parts of their mobility the model system is set to mimic the dynamic process of SNARE-mediated membrane fusion. ${ }^{[108]}$ 
Table 5-1 Collection of diffusion coefficients of proteins, lipids and docked vesicles in different model systems.

\begin{tabular}{|c|c|c|c|c|}
\hline Model system \& surface & Particle type & $\begin{array}{c}\text { Lipid mixture \& nominal } \\
\text { p/l ratio }\end{array}$ & $\begin{array}{l}D\left[\mu \mathrm{m}^{2} \mathrm{~s}^{-1}\right] \& \\
\text { technique }\end{array}$ & $\begin{array}{c}\text { Mobile } \\
\text { fraction } \\
{[\%]}\end{array}$ \\
\hline GUV & $\Delta$ N49-complex & $\begin{array}{l}\text { DOPC/POPE/POPS/Chol } \\
5: 2: 1: 2,1: 500\end{array}$ & $5 \pm 3$, FRAP & $100 \pm 7$ \\
\hline GUV & DPPE & $\begin{array}{l}\text { DOPC/POPE/POPS/Chol } \\
5: 2: 1: 2\end{array}$ & $9 \pm 3$, FRAP & $100 \pm 8$ \\
\hline GUV [115] & $\Delta$ N49-complex & $\begin{array}{l}\text { PC/PE/PS/Chol 5:2:2:1, } \\
1: 500\end{array}$ & 1.2, FRAP & \\
\hline GUV [115] & $\mathrm{PE}$ & PC/PE/PS/Chol 5:2:2:1 & 2.7, FRAP & \\
\hline GUV [134] & Syntaxin $1 \mathrm{~A}$ & $\begin{array}{l}\mathrm{PC} / \mathrm{PE} / \mathrm{PS} / \mathrm{PI} / \mathrm{Chol} \\
5: 2: 1: 1: 1,1: 300\end{array}$ & $2.5 \pm 0.5, \mathrm{FCS}$ & \\
\hline Synapse ${ }^{[145]}$ & Syntaxin $1 \mathrm{~A}$ & & $0.07, \mathrm{SPT}$ & 100 \\
\hline f-PSM ${ }^{[85]}$ & $\begin{array}{l}\text { Syntaxin 1A } \\
\text { Syx 1A-TMD }\end{array}$ & $\begin{array}{l}\text { DOPC/POPE/POPS/Chol } \\
5: 2: 1: 2,1: 500\end{array}$ & $\begin{array}{l}2.3 \pm 0.5, \mathrm{FCS} \\
3.4 \pm 0.2, \mathrm{FCS}\end{array}$ & \\
\hline f-PSM ${ }^{[85]}$ & DPPE & $\begin{array}{l}\text { DOPC/POPE/POPS/Chol } \\
5: 2: 1: 2\end{array}$ & $7.7 \pm 0.4, \mathrm{FCS}$ & \\
\hline s-PSM, SAM [95] & $\Delta$ N49-complex & $\begin{array}{l}\text { DOPC/POPE/POPS/Chol } \\
5: 2: 2: 1,1: 500\end{array}$ & $1 \pm 0.5$, FRAP & \\
\hline s-PSM, SAM ${ }^{[95]}$ & DPPE & $\begin{array}{l}\text { DOPC/POPE/POPS/Chol } \\
5: 2: 2: 1\end{array}$ & $2 \pm 1$, FRAP & \\
\hline s-PSM, SAM ${ }^{[86]}$ & Syx-TMD & $\begin{array}{l}\text { DOPC/POPE/POPS/Chol } \\
5: 2: 1: 2,1: 500\end{array}$ & $1 \pm 0.5$, FRAP & \\
\hline $\mathrm{S}$ and f-PSM combined, $\mathrm{SiO}_{2}$ & DOPE & DOPC/DOPS 7:3, 1:1000 & $3.8 \pm 0.4$, FRAP & \\
\hline $\mathrm{s}-\mathrm{PSM}\left(\mathrm{SiO}_{2}\right)^{[139]}$ & t-SNARE & DOPC/DOPS 7:3, 1:1000 & immobile, FRAP & 0 \\
\hline SLB (PEG + Langmuir) ${ }^{[78]}$ & t-SNARE & DOPC:DPS 9.7:0.3 1:500 & $0.75 \pm 0.2$, FRAP & $78 \pm 3$ \\
\hline SLB (PEG + Langmuir) ${ }^{[78]}$ & eggPE & DOPC:DPS 9.7:0.3 1:1000 & $0.65 \pm 0.05$, FRAP & $88 \pm 2$ \\
\hline SLB (quartz + Langmuir) ${ }^{[78]}$ & t-SNARE & $\begin{array}{l}\text { DOPC } \\
1: 500 \\
1: 1000\end{array}$ & $\begin{array}{l}0.9 \pm 0.1, \text { FRAP } \\
0.95 \pm 0.15, \text { FRAP }\end{array}$ & $\begin{array}{l}83 \pm 2 \\
68 \pm 3\end{array}$ \\
\hline SLB (quartz + Langmuir) ${ }^{[78]}$ & eggPE & DOPC 1:1000 & $1.45 \pm 0.25$, FRAP & $79 \pm 5$ \\
\hline SLB (quartz) ${ }^{[72]}$ & Syntaxin & egg PC, 1:4 & 0.07, FRAP & $3-7$ \\
\hline SLB (quartz) ${ }^{[72]}$ & egg PC & egg PC & $1-2$, FRAP & $50-70$ \\
\hline f-PSM ${ }^{[61]}$ & Syb-LUV & $\begin{array}{l}\text { DOPC/POPE/POPS/Chol } \\
5: 2: 1: 2\end{array}$ & $0.42 \pm 0.15, \mathrm{SPT}$ & \\
\hline f-PSM ${ }^{[87]}$ & CGs & & $0.34 \pm 0.06, \mathrm{SPT}$ & \\
\hline s-PSM ${ }^{[87]}$ & CGs & & $0.12 \pm 0.05, \mathrm{SPT}$ & \\
\hline
\end{tabular}




\subsection{Single-vesicle content release assay}

To get a better insight into the process of fusion pore formation in SNARE-mediated membrane fusion, fusion of individual vesicles with pore-spanning membranes was visualized by means of content release and high speed spinning disc confocal microscopy. Simultaneously measuring the influx of lipid into the 3D-structure of the vesicle enabled to investigate the interplay between fusion pore formation and lipid mixing as well as the quantification of different fusion pathways and kinetics.

\subsubsection{SNARE-mediated docking and fusion of LUVs with PSMs}

V-SNARE containing, content labeled LUVs were prepared by re-swelling of a syb 2 doped lipid film in SRB-solution (DOPC/POPE/POPS/cholesterol; 5/2/1/2 (n/n), nominal p/l 1:500, $43 \mathrm{~mm} \mathrm{SRB}$ ). Successful content inclusion and vesicle stability was proven with UV/Vis and bulk fluorescence spectroscopy. SNARE-mediated single-vesicle fusion with pore-spanning membranes harboring the fusion active t-SNARE acceptor complex $\Delta N 49$ (DOPC/POPE/POPS/cholesterol; 5/2/1/2 (n/n), nominal p/l 1:500) was imaged in dual color mode with a time resolution of $20.83 \mathrm{~ms}$ per frame. This high temporal resolution enabled the direct observation of content transfer upon fusion pore formation from the vesicular lumen into the open space underneath the f-PSM as well as the burst of vesicular content into the solution above the membrane (Figure 4.10). While, due to the rapid diffusion of SRB out of the open pore of the substrate, content transfer could only in some cases be directly visualized, the unique fluorescence intensity time trace of bursting vesicles allowed for the efficient discrimination of these two modes of fusion. Evaluating 1609 docked vesicles of 7 different preparations and 68 independent sets of experiments revealed a fusion efficiency of $52 \%$ with a $75 \%$ of chance of a complete content release and an extremely low probability of vesicle bursting of $0.003 \%$. The process of content transfer across the membrane could be visualized more efficiently when forming PSMs on porous substrates with closed cavities (Figure 4.27). Here the released SRB remains inside the confined $2^{\text {nd }}$ aqueous compartment underneath the f-PSM for several seconds and only slowly diffuses into neighboring cavities. Diffusion out of the pore is possible due to its shallow depth of $\sim 250 \mathrm{~nm}$ and shows slow kinetics due to the narrow water layer between the s-PSM and the gold/mercapto-hexanol functionalized pore rim. This direct observation of content transfer enabled by the $2^{\text {nd }}$ aqueous compartment underneath the f-PSM is a unique feature of PSMs and renders them exceptionally useful as a planar model system for the target presynaptic membrane. The process of content transfer was independently observed by 
Ramakrishnan et al. who entrapped a $\mathrm{Ca}^{2+}$ sensitive dye inside the lumen underneath the f-PSM and high concentrations of $\mathrm{Ca}^{2+}$ inside the vesicle population. ${ }^{[62]}$ They then monitored fusion pore formation as a spike in fluorescence due to release of $\mathrm{Ca}^{2+}$ into the cavity. These observations clearly distinguish PSMs from supported lipid membranes (SLBs) that are frequently used to study SNARE-mediated membrane fusion and lack a defined $2^{\text {nd }}$ aqueous compartment. ${ }^{[63,72-75,77]}$ While docking and fusion was repeatedly proven to be a SNARE-dependent process on PSMs, ${ }^{[62,85-87,95]}$ in early studies where SLBs were prepared by direct deposition of proteo-SUVs on glass surfaces fusion was found to be SNAP25 independent, ${ }^{[72,73]}$ thermally induced, ${ }^{[72]}$ or dependent on $\mathrm{Ca}^{2+}$ without the respective calcium sensor synaptotagmin $1 .{ }^{[48]}$ Even if fusion was SNARE-dependent, modeling the kinetics of content release during fusion experiments revealed that vesicles predominantly burst the content into the solution above the membrane (Figure 5.1 A). ${ }^{[74]}$ All these observations can be explained by the strong substrate-membrane interaction which leads to almost complete protein immobility, large immobile fractions (Table 5-1), ${ }^{[72]}$ and a very narrow $1 \mathrm{~nm}$ thick layer of water between the lipid bilayer and the glass surface. ${ }^{[148]}$ This lack of space for the content to be released in likely causes bursting of vesicles and also hinders the uptake of lipid material of vesicles. ${ }^{[63,74]}$ The problems of protein immobility and SNARE unspecificity were overcome by either introducing a polymer cushion between membrane and support ${ }^{[75,76]}$ or preparing SLBs in a two-step process of $1^{\text {st }}$ a Langmuir-Blodgett transfer followed by proteo-SUV deposition and thus decoupling the membrane from the solid support. ${ }^{[77,78]}$ While protein mobility was increased and fusion SNARE-dependent, the target membrane was, especially in the case of a Langmuir-Blodgett transfer, still in direct contact with the underlying substrate. This could influence the capability of the SLB to uptake incoming lipid material and interior solution from the fusing vesicle as well as the content release kinetics. ${ }^{[63]}$ Additionally, content transfer can only be validated indirectly and needs the comparison of each individual fluorescence intensity time trace with diffusion models that were derived using input variables that do not match the experimental conditions (Figure 5.1 B). ${ }^{[82]}$ 
A

Calcein Data (1 ms/frame) vs Models

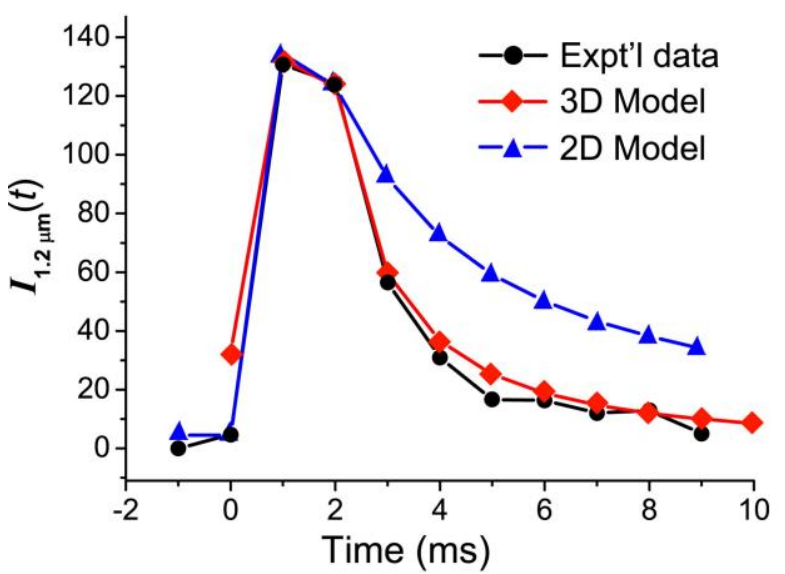

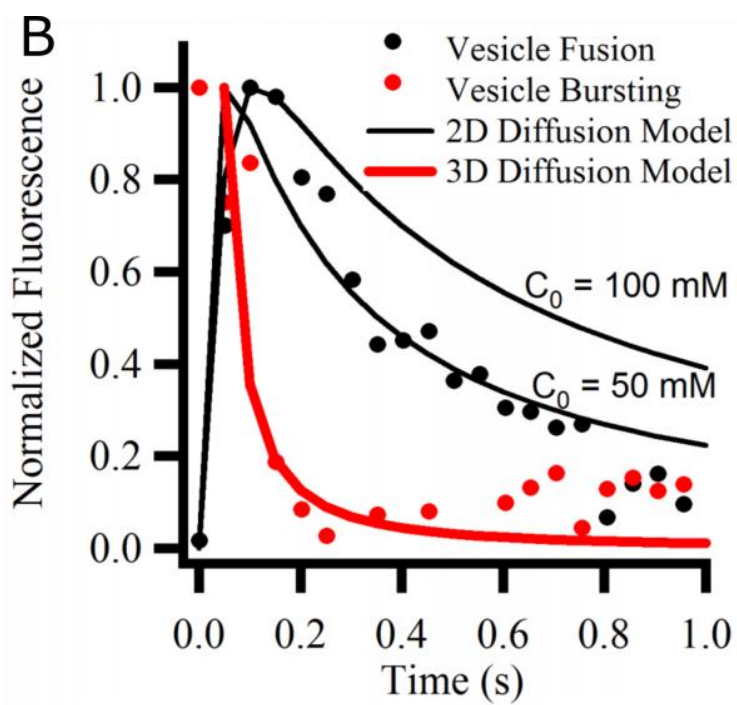

Figure 5.1 2D and 3D diffusion model to determine direction of content release in SLB based fusion setups. (A) Fusion of calcein filled vesicles with SLBs that were formed by direct adsorption of proteo-SUVs to glass leads to the burst of content into the solution above the membrane. Figure adapted from Wang et al. ${ }^{[74]}$ (B) Dye diffusion out of the confocal volume of vesicles filled with $100 \mathrm{mM}$ SRB fusing with a SLB (black dots) and bursting on a supported lipid monolayer (red dots) formed by the Langmuir-Blodgett transfer-technique. The comparison of the release kinetic of fusing vesicles with the 2D diffusion model for $50 \mathrm{mM}$ SRB proves the content transfer across the SLB during the fusion process. Figure adapted from Kreutzberger et al. ${ }^{[82]}$

\section{Docking behavior of proteo-LUVs}

However, while the $2^{\text {nd }}$ aqueous compartment underneath the f-PSM enabled the direct observation of content transfer upon pore formation it has to be noted that $99 \%$ of vesicles docked immobile to and fused with the s-PSM. With a surface ratio of $6: 4$ between solid supported and freestanding parts LUVs clearly show a tendency to dock to the pore rim, more precisely to the edge of the pore which is in accordance to previous studies. ${ }^{[61,149]}$ While this seems to have no influence on the capability of the vesicle to transfer its content into the aqueous space below the f-PSM, the question remains what causes this behavior. Even under the assumption that the first formal contact with the PSM occurs randomly, vesicle immobilization at the pore edge inevitably leads to accumulation of vesicles that are docked to the s-PSM. Such immobilization of v-SNARE doped vesicles on t-SNARE supported membranes is a feature described by all single-vesicle fusion assays that are based on pure SLBs ${ }^{[48,72-75,77]}$ and was independently observed by Ramakrishnan et al. at the edge of the pore rim of PSMs. ${ }^{[139]}$ Different explanations are discussed in literature that cause this immobilization all of which are correlated to the underlying surface of supported membranes. Liu et al. observed $\sim 1 \mu \mathrm{m}$ large defects inside the SLB that are formed during the spreading process of SUVs and to which v-SNARE vesicles docked effectively. ${ }^{[73]}$ As PSMs are formed from GUVs and as such from one continuous bilayer these defects are unlikely to exist on the pore rim and would probably lead to rupture of the f-PSM. A different explanation is that a conformal contact of the docked 
LUV with the supported membrane induced by long ranging interactions with the solid support causes vesicle immobilization. ${ }^{[86]}$ This effect would be comparably large on PSMs formed on a gold/MH functionalized surface due to the large Hamaker constant of gold compared to glass $/ \mathrm{SiO}_{2} \cdot{ }^{[150]}$ Lastly, interaction of v-SNAREs with immobile acceptor complex inside the s-PSMs (see Chapter 5.2) caused by interactions of proteins with the solid support could lead to vesicle immobilization. This is corroborated by the study of Ramakrishnan et al. who correlated vesicle immobilization at the s-PSM with the interaction of v-SNAREs with immobile t-SNARE aggregates that were imaged at the edge of the pore rim. ${ }^{[139]}$ Because of the inverted microscopy setup used to monitor fusion in their study in combination with the opaqueness of the pore grid large fractions of the pore rim are not visualized and consequently the ratio of f-PSM and s-PSM docked vesicles not quantified. Nevertheless, it can be assumed that also in this study a large fraction of vesicles, while undetected, at least dock to and probably fuse with the s-PSM especially as proteins in this setup proved to be entirely immobile on the pore rim. Large improvements in the labeling procedure of the full acceptor SNARE complex and the reconstitution procedure made during this work enabled, for the first time, to directly image reconstituted $\triangle \mathrm{N} 49$-complex inside PSMs. Indeed, aforementioned t-SNARE aggregates were also found here, often times forming at the edge of the pore (Figure 5.2 B), and proved to be entirely immobile. The fact that other PSMs showed no aggregates (Figure 5.2 A) could be explained by variations in cluster size, as smaller clusters would not be visible due to the fluorescence quenching of the underlying gold surface at distances $<15 \mathrm{~nm} .{ }^{[93,99]}$ In addition, FRAP experiments suggest that a significant fraction of proteins is, independent of the existence of visible t-SNARE aggregates at the pore edge, immobile on the pore rim. Notably, large protein clusters were never observed inside the f-PSM but could already be observed inside the membrane of freely floating GUVs (Figure 4.3 B) and showed full mobility. This leads to the assumption that t-SNARE clusters are formed during the reconstitution procedure. Generally, $\mathrm{t}$-SNARE clustering is thought to originate from homotypic interactions of syntaxin $1 \mathrm{~A}$ and its transmembrane regions. ${ }^{[132,151-153]}$ Both varying protein densities inside GUVs and differences in concentration of lipids between vesicles can enhance this process. For example, Liu et al. reported large, AFM resistant t-SNARE aggregates in SLBs when using high protein densities that disappear for low $\mathrm{p} / 1$ ratios. ${ }^{[73]}$ On the other hand, varying concentrations of cholesterol inside the GUV membrane, which is thought to enhance syx 1A clustering, could cause acceptor complex aggregation. ${ }^{[147,154]}$ If the GUV is then spread on the porous substrate immobilization of protein at the pore rim would lead to a higher concentration of SNAREs at the edge of the pore. This might further increase the tendency of vesicles to dock to this specific part of the 
PSM as higher protein densities lead to a higher chance for a first contact between v- and t-SNAREs to occur.

In summary, the preferred docking of vesicles concomitant with their immobilization at the pore rim could be a convolution of increased concentrations of immobile acceptor complex and the conformal contact between vesicles and s-PSM induced by the large Hamaker constant of the gold functionalization.
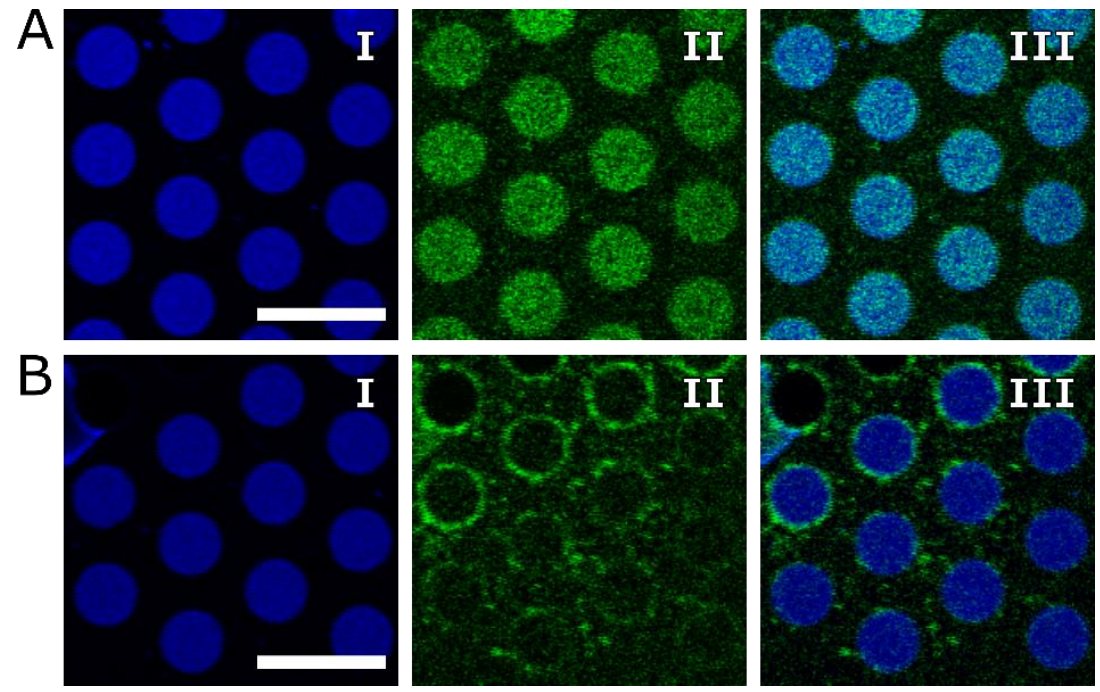

Figure 5.2 Fluorescence micrograph of Atto390-DPPE labeled PSMs (A-B, I, blue), $\Delta$ N49-Atto488 complex (A$\mathrm{B}$, II, green), and overlay of the two channels (A-B, III). (A) The protein can be homogeneously distributed inside the f-PSM with no visible aggregates. (B) Protein aggregation at the pore edge and on the pore rim is visible as increased fluorescence intensity. Scale bars $=10 \mu \mathrm{m}$. Micrograph A-B II were obtained by summing up 300 individual snapshots recorded over $20 \mathrm{~s}$, showing the immobility of visible aggregates.

\section{Docking of vesicles to the f-PSM}

As described in Chapter 4.4.6 proteo-LUVs that interact with the f-PSM, in contrast to those immobilized at the s-PSM, retain their mobility throughout the whole docking process with a mean diffusion coefficient of $D=0.15 \mu \mathrm{m}^{2} \mathrm{~s}^{-1}$. This diffusion coefficient is by one order of magnitude lower compared to the free Syx $1 \mathrm{~A}\left(2.3 \pm 0.5 \mu \mathrm{m}^{2} \mathrm{~s}^{-1}\right)$ inside the f-PSM. This decrease in mobility which was also observed by Ramakrishnan et al. could be explained by the interaction of multiple SNARE-complexes during the docking and fusion process. ${ }^{[62]}$ Indeed, while the exact number is of constant debate, it is usually assumed that several SNARE complexes are necessary to enable vesicle fusion ${ }^{[75,77,155-157]}$ and that this number is increased with larger vesicle diameters. ${ }^{[30]}$ Additionally, the process of SNARE zippering leads to the displacement of the water layer between the opposing membrane and the formation of a large circular contact area that is discussed to be rigid. ${ }^{[56,158]}$ The induced larger frictional coupling between the two membranes could further decrease the mobility of docked LUVs. 
Another important observation is that, in contrast to both lipids and proteins, LUVs show confined diffusion inside one f-PSM, which is reflected by the saturation of the extracted mean square displacement (MSD) at $\sim 0.3 \mu \mathrm{m}^{2}$ (Figure $4.25 \mathrm{~A}$ ). It is likely that the membrane geometry at the pore rim-region is acting as a barrier that is only effecting the docked LUVs. Previous studies showed that the membrane bends in this region about $100 \mathrm{~nm}$ into the pore probably following the gold/MH functionalization (Figure $4.13 \mathrm{~A}) .^{[159,160]}$ The very large contact area between the vesicle and target membrane would thus experience deformation if the vesicle comes into close proximity to the curved edge of the pore. As such a deformation is energetically unfavorable the vesicle would not be able to diffuse over the pore edge. However, if the vesicle overcomes this barrier, it is, as mentioned above, immediately immobilized due to interactions with immobile t-SNAREs or the underlying gold surface. This behavior of confined diffusion and immobilization at the pore edge was also observed in previous studies on PSMs. ${ }^{[62,86,139]}$ However, replacing synthetic vesicles with natural chromaffine granules (CGs) prevented vesicle immobilization at the pore rim and enabled free diffusion over the edge of the pore. ${ }^{[87]}$ The dense protein shell of the chromaffine granules is thought to reduce the contact area between the vesicle and target membrane and thus further uncouples the vesicle from the s-PSM. Consequently, as CGs are mobile on the s-PSM, complete immobilization of synthetic vesicles at the pore edge is more likely caused due to interactions with the underlying substrate and not induced by interaction of v-SNAREs with immobile t-SNARE aggregates. Furthermore, CGs remained mobile even after lipid mixing was occurred on either part of the PSM. While CGs diffused inside the f-PSM prior to lipid mixing with $D=0.34 \pm 0.06 \mu \mathrm{m}^{2} \mathrm{~s}^{-1}$ their mobility was reduced by a factor of 2.8 on the s-PSM (Table 5-1). The decrease in mobility is probably caused by the remaining influence of the gold functionalization and generally lower mobility of t-SNAREs inside the s-PSM compared to the f-PSM.

Notably, the mean diffusion coefficient of CGs on the f-PSM quantified by Hubrich et al. is larger than the one determined in the present work. Moreover, Kuhlmann et al. determined a mean diffusion coefficient of synthetic, $240 \mathrm{~nm}$ sized LUVs of $D=0.42 \mu^{2} \mathrm{~s}^{-1}$ and a MSD that usually reached values of $\sim 1.3 \mu \mathrm{m}^{2}$. Both the decreased MSD and diffusion coefficient can be explained by a decrease in pore diameter from $5 \mu \mathrm{m}$ in the two studies to $1.2 \mu \mathrm{m}$ in the present work. This is endorsed by the work of Raccis et al. who investigated the effect of increased confinement on the diffusion of spherical particles in porous nanostructures and correlated the observed decrease in mobility with increased interactions of the tracer molecules with the walls of the cavity. ${ }^{[161]}$ The strong dependence between cage size and diffusion 
behavior is also reflected in the comparably low diffusion coefficient of $D=5 \cdot 10^{-5} \mu \mathrm{m}^{2} \mathrm{~s}^{-1}$ found for docked synaptic vesicles at the presynaptic membrane with a cage size of $50 \mathrm{~nm} .{ }^{[162]}$ The fact that vesicles dock mobile to the f-PSM highlights their advantage to solid supported based membranes to mimic the dynamic process of SNARE-mediated membrane fusion. However, the low statistics due to immobilization of vesicles at the pore rim induced by the solid support makes it challenging to gather statistically relevant information. Recent studies using CGs suggest that vesicle mobility might be regained by the introduction of PEG-linkers to the membrane of synthetic proteo-liposomes thus mimicking the crowded environment of the membrane of natural vesicles such as CGs.

\section{Docking efficiency}

Another important observation about the docking behavior of vesicles to PSMs was the strongly varying amount of vesicles docked to PSMs. By accounting for differences in membrane patch sizes a mean docking efficiency of $0.43 \pm 0.56$ vesicles per $\mu \mathrm{m}^{2}$ was extracted. Since the reconstitution efficiency of $\Delta \mathrm{N} 49$-complex into GUVs exhibited similar variations with $R=26 \pm 24 \%$, which reflects an effective p/l ratio of $1 / 1900$ or $\sim 800$ complexes per $\mu \mathrm{m}^{2}$, changing docking efficiencies most likely are a function of accessible acceptor complex inside the PSM. A study of Domanska et al. supports this hypothesis who reported an increase in docked liposomes per $\mu \mathrm{m}^{2}$ when increasing the concentration of acceptor complex inside the target membrane that reached a plateau at $\sim 500$ complexes per $\mu \mathrm{m}^{2}{ }^{2}{ }^{[7]]}$ Interestingly, docking efficiencies to membranes in SLB-based setups show significantly less variations which can be explained by the quantitative reconstitution efficiency of proteins into SUVs that are the starting material for the preparations of the acceptor membrane. ${ }^{[53,73,164]}$ This further supports the hypothesis that changing docking efficiencies observed in this work directly correlate with varying protein concentrations inside the PSMs. This raises the question, whether or not also the fusion efficiency is influenced by these variations in acceptor complex density. For example, a study of Ji et al. suggests a cooperative effect of t-SNARE density and fusion probability in a bulk fusion setup. ${ }^{[165]}$ Surprisingly, fusion curves do not reach a plateau in this study which suggests that the presumably lower fusion efficiency might actually be a kinetic effect driven by an increased probability of docking through SNARE-interactions at higher protein densities. This is in agreement with the bulk fusion study of Hernandez et al. who reported identical fusion efficiencies for different syb 2 and $\Delta \mathrm{N} 49$ densities inside SUVs while kinetics decreased for lower densities. ${ }^{[30]}$ To test the influence of SNARE densities in the 
present work, fusion efficiencies of vesicles with individual PSMs were correlated with the respective determined docking efficiency (Figure 5.3). The population scatters strongly for lower efficiencies/total number of docked vesicles, as outliers have a stronger influence on the fusion efficiency, and converges for higher docking efficiencies to the global mean fusion probability of $52 \%$. With a correlation coefficient of $\rho=0.0584$ the increased docking rate due to higher protein concentration clearly does not change the probability for vesicles to fuse with the membrane. In summary, higher protein densities positively correlate with increasing docking efficiencies while not influencing the fusion efficiency of the process.

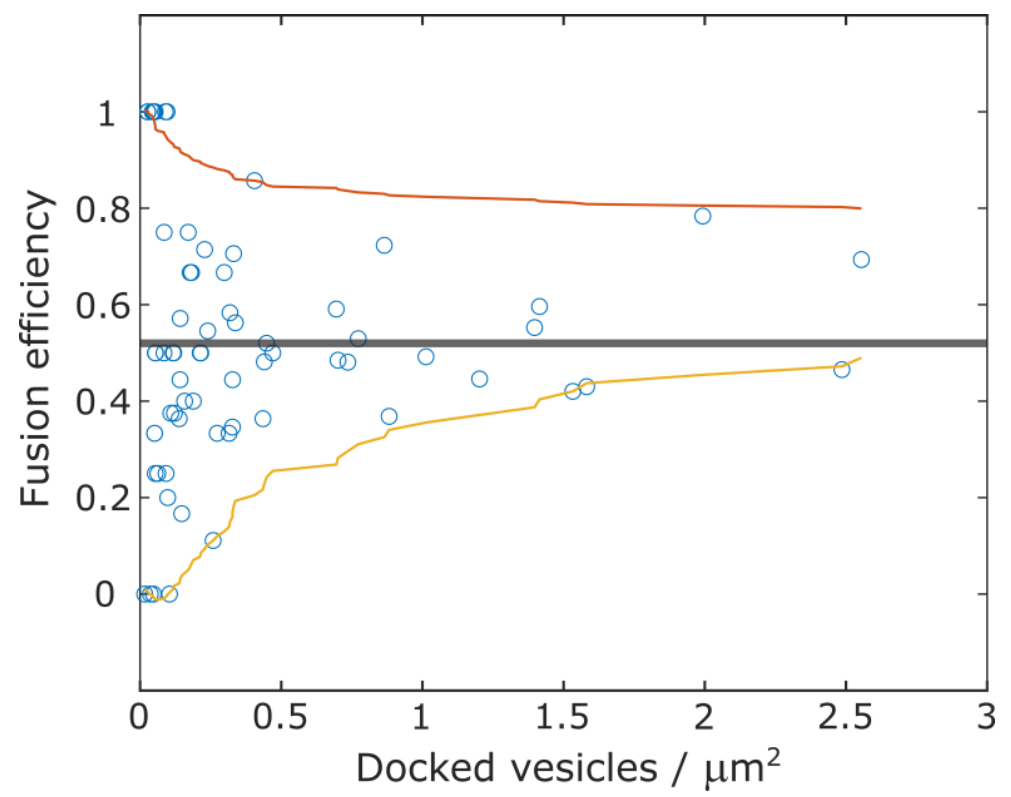

Figure 5.3 Scatterplot of the docking efficiency against the mean fusion efficiency of vesicles with the respective membrane. The data converges for large amounts of docked vesicles per $\mu^{2}$ to the global mean fusion probability of $52 \%$ (horizontal grey line) highlighted by the envelopes of the population with a correlation coefficient of $\rho=0.0584$.

\subsubsection{Docking times and fusion efficiencies of proteo-LUVs}

Two important kinetic observables in the process of SNARE-mediated membrane fusion are the efficiency with which vesicles fuse and the time between the first formal contact of the vesicle with the target membrane and the moment of fusion pore formation, also called docking time. Both are commonly used to describe the capability of a model system to mimic the fusion process of vesicles in live cells, however, especially the latter one is discussed to be influenced by various factors such as lipid composition, used acceptor complex constructs, vesicle size and the type of target membrane. As a result, docking times vary greatly between various single-vesicle model systems (Table 5-2). While these factors make it difficult to compare docking times and fusion efficiencies quantitatively, this paragraph will focus on the discussion 
of fusion kinetics of this work in context with those of other, commonly used model systems to shed some light on possible reasons for major variations.

In this work, docking times were extracted from 840 fusing vesicles, plotted, and a mono-exponential decay was fit to the data resulting in an average docking lifetime of $\tau_{\text {docking }}=65 \pm 4 \mathrm{~s}$ with an overall fusion efficiency of $52 \%$. Previous studies on pore-spanning membranes reported very similar fusion efficiencies. ${ }^{[62,85,86]}$ While fusion assays based on pure SLBs exhibited very low $\mathrm{FE},{ }^{[48]}$ the introduction of PEG-linkers and Langmuir-SLB formation drastically increased the fusion probability to similar values of $\sim 50 \%{ }^{[53,75]}$

Previous studies performed on PSMs reported on mean docking times ranging between 13 and 44 seconds for vesicles fusing with the s-PSM that are independent of vesicle diameter and do not change if synthetic vesicles are exchanged by natural CGs. ${ }^{[85-87]}$ The slightly larger values found in the present work can be explained by a longer observation time which leads to a shift to long docking times as it is more likely that the imaged vesicle fuses within the observation window. Notably, vesicles show similar fusion kinetics on the f-PSM compared to those fusing with the s-PSM. While a docking time could not be quantified due to low statistics, exemplarily fusion events suggest that it is in the same range of several tens of seconds. This is in good agreement with the study of Kuhlman et al., who extracted docking times of vesicles fusing with the f-PSM of up to $60 \mathrm{~s}{ }^{[86]}$ The large docking times of tens of seconds are supposedly the longest docking times for SNARE-mediated single-vesicle fusion assays reported in literature and several orders of magnitude longer than kinetics observed in vivo. ${ }^{[166]}$ They can be explained by $1^{\text {st }}$ strong Van der Waal interactions of the docked vesicle with the underlying gold surface due to its high Hamaker constant. ${ }^{[150]}$ Secondly several studies show that the displacement of the small, stabilizing syb fragment from the $\Delta \mathrm{N} 49$-complex has a half-life time of 19-20 s. ${ }^{[51,167,168]}$ This explains the shorter docking times reported by Ramakrishnan et al. of 0.6-4 s who used a native 1:1 complex of syx 1A (including the Habc domain) and SNAP25 that was formed by co-expression of the two proteins. ${ }^{[62]}$ These kinetics are also in good agreement with the study of Karatekin et al. who reported mean docking times of $0.16 \mathrm{~s}$ on PEG-SLBs using a similar fusion machinery. However, other fusion assays that are based on Langmuir-SLBs or GUVs as target membranes reconstituted the same $\Delta \mathrm{N} 49$-complex used in the present study and measured docking times reduced by 1-3 orders of magnitude ranging from $5 \mathrm{~s}$ down to $20 \mathrm{~ms} .{ }^{[33,77,115]}$ It is puzzling that the half-life time of displacing the small syb fragment of 19-20 s enables such short docking times. While it is possible that, as mentioned above, the large Hamaker constant of the gold surface deposited on porous substrates leads to 
the observed long docking times on s-PSMs, this does not explain the equally long docking times found on f-PSMs. ${ }^{[86]}$ Thus, one would at least suspect a shift to larger docking times when the $\Delta \mathrm{N} 49$-complex is displaced by a more native 1:1 complex within the same model system. However, a study of Kreutzberger et al. compared fusion kinetics using the $\Delta$ N49-complex with two syx:SNAP25 1:1 constructs and surprisingly monitored identical fusion kinetics. ${ }^{[53]}$ While one could argue that differences in protein densities used in this study can cause these changes in docking times, as several studies suggest an optimal p/l ratio for syb 2 of $1 / 200,{ }^{[51,77]}$ a different study reports for an optimal $\mathrm{p} / 1$ ratio of $1 / 600 .{ }^{[75]}$ Additionally, since the observed docking times in the present work vary significantly from those published by Witkowska et al who used identical p/l ratios, the same acceptor complex type and a very similar lipid composition, the effect of syb 2 densities can be ruled out as well. ${ }^{[115]}$ The results of the present work show that also variations in t-SNARE acceptor complex density do not influence docking times significantly (Figure 4.12 A, $\rho=0.137$ ). In addition, mean fusion kinetics of assays based on surface adhered vesicles are usually $>250 \mathrm{~ms}$ up to $6.8 \mathrm{~s} .{ }^{[64,68,69,155]}$ All these observations raise the question to which extent fusion might be induced by other factors in some of the above mentioned model systems such as remaining detergent, small artifacts inside the membranes, the underlying solid support or laser induced heating as it was shown in the study of Bowen et $a l .{ }^{[72]}$

Next to docking times also the number of hidden reaction steps of the fusion process can be extracted by data fitting with the gamma distribution ${ }^{[169]}$ or similar models. ${ }^{[77]}$ Again, independent of the used acceptor complex, different numbers of reaction steps were found ranging from a 1-step process up to 9 independent steps. ${ }^{[53,61,68,82,95,105,149,163]}$ It is thus questionable, if quantitative values can be extracted from the shape of the docking time distribution.

All these factors lead to the hypothesis that docking times should not be used as a measure for the effectiveness of the respective model system to mimic the process of SNARE-mediated fusion and neither to draw quantitative conclusions such as the number of SNAREs involved in the fusion process. However, they are powerful tools to investigate the influence of different variables such as regulatory proteins or vesicle sizes on the fusion kinetics. For example, Kreutzberger et al. observed in a beautiful study using isolated synaptic vesicles $(\sim 40 \mathrm{~nm})$, dense core vesicles, and insulin vesicles $(\sim 200 \mathrm{~nm})$ that the docking time increases from 0.227 up to $9.1 \mathrm{~s}$ with increase in vesicle diameter. ${ }^{[71]}$ This size-dependent fusion kinetic was also observed by Hernandez et al. for both bulk and single-vesicle fusion experiments and is 
commonly correlated with a change in membrane curvature as shown in a simulation study of Malinin et al. ${ }^{[30,60]}$ Surprisingly, Kuhlmann did not observe changes in the fusion kinetics when comparing docking times of vesicles with diameters of 100 and $240 \mathrm{~nm}$ on PSMs. ${ }^{[61]}$ The same was observed in the present study where the vesicle mean intensity, which can be seen as an indicator for apparent vesicle size, shows no correlation with the respective docking time of the LUV (Figure 4.12 B, $\rho=0.0727$ ). It is, however, possible that the model system of PSMs, due to the inherently larger docking times, is not capable of monitoring the above mentioned changes. 
Table 5-2 Collection of docking times ( $t_{\text {docking }}$ ) and fusion efficiencies of different single-vesicle fusion assays using the minimal fusion machinery without regulatory proteins. If not stated otherwise, measurements were performed at room temperature. If not specified, target membrane and vesicle lipid composition is identical.

\begin{tabular}{|c|c|c|}
\hline t-SNARE membrane & v-SNARE membrane & $t_{\text {docking }} \&$ fusion efficiency \\
\hline \multicolumn{3}{|l|}{$\underline{\mathbf{S L B}^{[48]}}$} \\
\hline PC, Syx $1 \mathrm{~A}+\mathrm{SNAP} 25, \mathrm{p} / 1 \mathrm{NaN}$ & SUVs, PC/PS/PE (8.2:1.5:0.3), & $0-50 \mathrm{~s}, 0.35 \%$ \\
\hline Syx 1A- Habc +SNAP25, p/1 NaN & syb 2, p/1 NaN & $0-50 \mathrm{~s}, 10.5 \%$ \\
\hline \multicolumn{3}{|l|}{ PEG-SLB ${ }^{[75]}$} \\
\hline $\begin{array}{l}\text { PC/PS/PEG-PE (8:1.5:0.5), Syx } \\
\text { 1A+SNAP25, 1:10000 }\end{array}$ & SUVs, syb $2,1: 120,27^{\circ} \mathrm{C}$ & $0.16 \mathrm{~s}, 50 \%$ \\
\hline \multicolumn{3}{|l|}{ Langmuir-SLB ${ }^{[77]}$} \\
\hline $\mathrm{PC} / \mathrm{Chol}(8: 2), \Delta \mathrm{N} 49,1: 3000$ & SUVs, syb 2, 1:200 & $\begin{array}{l}0.02-0.25 \mathrm{~s} \\
(37 \%>0.25 \mathrm{~s}), 43 \%\end{array}$ \\
\hline \multicolumn{3}{|l|}{ Langmuir-SLB ${ }^{[53]}$} \\
\hline $\mathrm{PC} / \mathrm{Chol}(8: 2), \Delta \mathrm{N} 49,1: 3000$ & SUVs, syb 2, 1:400 & $0.07-5 \mathrm{~s}, 32 \%$ \\
\hline Syx 1A- Habc:SNAP 25a, 1:3000 & & $0.07-5 \mathrm{~s}, 38 \%$ \\
\hline Syx 1A- Habc:d-SNAP25, 1:3000 & & $0.07-5 \mathrm{~s}, 39 \%$ \\
\hline \multicolumn{3}{|l|}{$\underline{\mathbf{S}-\mathbf{P S M}}{ }^{[87]}$} \\
\hline $\begin{array}{l}\Delta \mathrm{N} 49, \mathrm{PC} / \mathrm{PE} / \mathrm{PS} / \mathrm{Chol} / \mathrm{PI}(4,5) \mathrm{P}_{2} \\
(4.8: 2: 1: 2: 0.2)\end{array}$ & $\begin{array}{l}240 \mathrm{~nm} \text { LUVs, PC/PE/PS/Chol } \\
(5: 2: 1: 2), \text { syb } 2,1: 500\end{array}$ & $44 \mathrm{~s}, 92 \%$ \\
\hline \multicolumn{3}{|l|}{$\underline{\mathbf{S - P S M}}{ }^{[61,86]}$} \\
\hline \multirow[t]{2}{*}{$\Delta \mathrm{N} 49, \mathrm{PC} / \mathrm{PE} / \mathrm{PS} / \mathrm{Chol}(5: 2: 1: 2)$} & 90 nm LUVs, syb 2, 1:500 & $16.7 \mathrm{~s}, 47 \%$ \\
\hline & $240 \mathrm{~nm}$ LUVs, syb 2, 1:500 & $12.5 \mathrm{~s}, 47 \%$ \\
\hline$\underline{\mathbf{f}-\mathbf{P S M}{ }^{[86]}}$ & $240 \mathrm{~nm}$ LUVs, syb 2, 1:500 & $0-60 \mathrm{~s}, \mathrm{NaN}$ \\
\hline$\underline{\mathbf{f}-\mathbf{P S M}}{ }^{[62]}$ & & \\
\hline $\begin{array}{l}\text { PC/PC (7:3), Syx 1A+SNAP25, } \\
1: 1000^{[62]}\end{array}$ & $\begin{array}{l}\text { SUVs, PC/PS/PE (8.2:1.5:0.3), } \\
\operatorname{syb} 2,1: 400\end{array}$ & $0.6-4 \mathrm{~s}, 60 \%$ \\
\hline GUVs ${ }^{[115]}$ & & \\
\hline $\begin{array}{l}\mathrm{PC} / \mathrm{PE} / \mathrm{PS} / \text { Chol }(5: 2: 2: 1), \Delta \mathrm{N} 49, \\
1: 1000\end{array}$ & 100 nm LUVs, syb 2, 1:500 & $0.03 \mathrm{~s}, \mathrm{NaN}, 0.5 \mathrm{mM} \mathrm{Mg}^{2+}$ \\
\hline
\end{tabular}

\subsubsection{Pathways and kinetics of vesicle fusion}

While the detection of content release provided information about the time point of fusion pore formation it was also used to extract the kinetics of the content release process. Additionally, as the target membrane fluorescence intensity indicates the moment when lipid mixing between the vesicle and PSM occurs, it was used to investigate the existence of a hemifusion structure and the lifetimes of 3D postfusion structures. By simultaneously monitoring both processes with high time resolution the interplay of fusion pore formation and lipid mixing enabled the 
quantification of various fusion pathways and the lag time between lipid mixing and pore opening.

\section{Fusion pathways}

Out of $840(52 \%)$ fusing vesicles $75 \%$ released the content dye completely after fusion pore formation while the fusion pore closed again in the remaining $25 \%$. Remarkably, after a first incomplete release the fusion pore could open again with a likelihood of $51 \%$. This dynamic opening and closing of a fusion pore is also termed as flickering fusion pore and resulted in a complete release as the final state for $25 \%$ of this population. Due to the quenching of fluorescence at distances $<15 \mathrm{~nm}$ to the gold surface and the mean vesicle diameter of $240 \mathrm{~nm}$, the mixing of lipids of the unlabeled vesicular membrane and the labeled target membrane was visible as an increase in Atto655-DPPE fluorescence. ${ }^{[86,93]}$ In summary, $24 \pm 11 \%$ of vesicles fused without detectable lipid mixing while in $28 \pm 16 \%$ of events lipid diffusion over a fusion stalk was concomitant to content release and could result in a stable or unstable 3D-postfusion structure. Self-explanatory this 3D-postfusion structure, also called $\Omega$-shape, could not collapse into the target membrane if the fusion pore closed and the release was incomplete. In this case the vesicle likely arrests in a hemifused state as depicted in Figure 4.14 A that can also be observed if the content release was complete $(8 \pm 7 \%)$. This is supported by the hypothesis that the process of fusion pore closing is not a reversal of the opening step and that the pore can reseal even if it is relatively large. ${ }^{[170,171]}$ This suggests that the vesicle is hindered in its full release and/or collapse if the lifetime of the fusion pore is too short. As expected, a complete content release with visible lipid mixing also resulted in the full collapse of the vesicle into the PSM which is reflected by an instable $\Omega$-shape $(7 \pm 7 \%)$ with varying lifetimes. To understand the underlying mechanisms that control the observed fusion pathways of syb 2-LUVs the docking efficiency of vesicles to each of the 68 PSMs as a function of t-SNARE densities were correlated with the occurrence of the respective mode of fusion. The amount of accessible acceptor complex has, deduced from the correlation coefficients summarized in Table 4-2, no influence on the fusion pathway. Whether the v-SNARE density has an impact is, however, unknown. Besides changing $\mathrm{p} / \mathrm{l}$ ratios inside the target membrane another changing variable is the size of the fusing LUV with a mean diameter of $240 \pm 100 \mathrm{~nm} \cdot{ }^{[86]}$ One finds that only $2 \%$ of LUVs that fuse without visible lipid mixing exhibit fluorescence intensities $>0.2$ au while $26 \%$ of vesicles that release their content incompletely and $7 \%$ that show a complete release and visible lipid mixing over a fusion stalk are above this threshold. Since the lower end of vesicle intensities is present in all populations this effect cannot be caused by limitations in the 
sensitivity of the setup to detect the lipid influx into the 3D structure of smaller vesicles. Hence a larger vesicle size likely increases the possibility of a 3D postfusion structure and in particular an incomplete content release. This supports the hypothesis that an incomplete release is caused by the lifetime of the fusion pore being too short for a full release. An alternative explanation for an incomplete release which could also explain the increased fluorescence would be the fusion of vesicle aggregates or a LUV that is either multilamellar or contains vesicle inclusion bodies that could be formed during the extrusion process. ${ }^{[172]}$ Two theoretical considerations about the expected (and observed) fluorescence intensity time traces disprove this possibility. First, vesicle aggregates would not lead to a stable 3D postfusion structure since the lipids between the non-fusing vesicle aggregate and the target membrane would not mix. Furthermore, Figure 5.4 illustrates the theoretical fluorescence intensity time traces of a vesicle containing an inclusion and fusing via a flickering fusion pore. Inevitably, the $2^{\text {nd }}$ fusion step would lead to the content to be released into the bulk solution above the membrane. However, a concomitant peak in fluorescence intensity was not reported for vesicles fusing via such a flickering fusion pore. Thus, at the same time these two considerations also prove that the fusion mode termed "flickering fusion pore" indeed originates from a unilamellar vesicle fusing via consecutive openings and closings of a SNARE-mediated fusion pore.

It is also possible that changes in the used lipid mixture affect the detected fusion pathway, especially the amount of incomplete release, as for example higher concentrations of cholesterol were found to extend the open state of the fusion pore. ${ }^{[36]}$

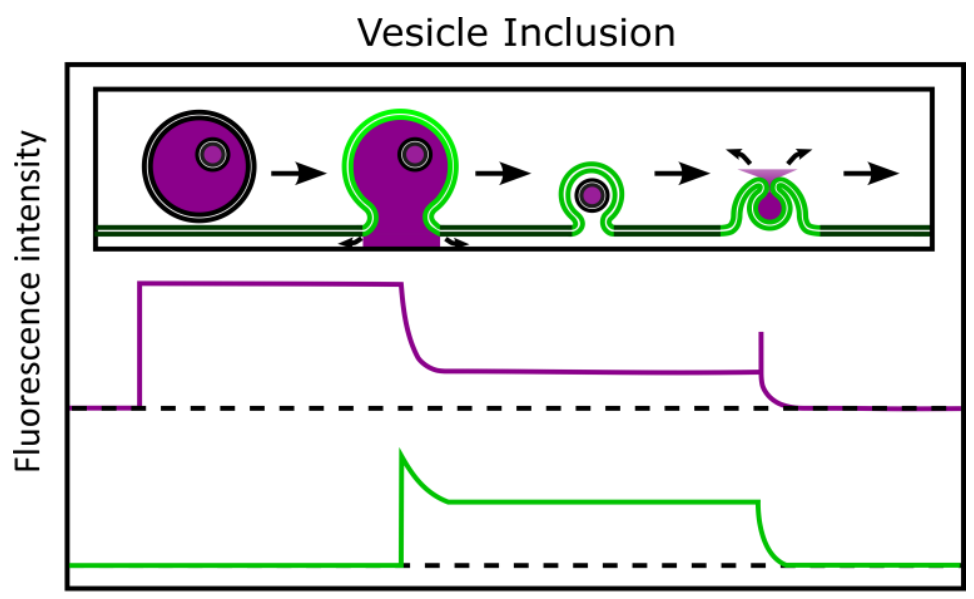

Time

Figure 5.4 Theoretical fluorescence intensity time traces of content marker SRB (magenta) and target membrane fluorescence (green) of a vesicle containing an inclusion. A second theoretical fusion pore formation would result in the release of SRB into the bulk solution above the membrane visible as a peak in the respective fluorescence intensity time trace. 


\section{Off-pathway hemifusion}

Interestingly, a process where only the two outer leaflets mix without the opening of a fusion pore, also called dead-end hemifusion, was negligible in this study. In contrast to productive on-pathway hemifusion, hemifusion as an off-pathway product is usually discussed as an physiologically irrelevant measuring $\operatorname{artifact}^{[33-35,173-175]}$ and thought to eventually occur due to incomplete zippering of the SNARE complex. ${ }^{[176]}$ Nevertheless, stalled hemifusion was reported in different single-vesicle fusion assays with e.g. $\sim 60 \%$ of hemifusion observed by Kreutzberger et al. at $20 \%$ cholesterol, where it negatively correlated with increased cholesterol concentration inside the target membrane. ${ }^{[67,82,177]}$ Controversially, in a follow up study with identical target membrane composition and a less complex vesicle lipid mixture <2\% hemifusion was detected. ${ }^{[53]}$ A recent study of Kuhlmann et al. that solely used lipid mixing as an indicator for fusion found 4-9\% of the vesicle population to likely show dead-end hemifusion. ${ }^{\left[{ }^{86]}\right.}$ The results of the present work, however, suggest that incomplete lipid mixing in these events was concomitant with a rapid opening and closing of the fusion pore that did not allow for complete lipid exchange between the two opposing bilayers to occur. This highlights the importance of the detection of content mixing for the interpretation of single-vesicle fluorescence intensity time traces and the discrimination of fusion pathways. The fact that negligible amounts of off-pathway hemifusion were observed using PSMs as the target membrane demonstrate that they are a useful tool to mimic key physiologic features of SNARE-mediated membrane fusion.

\section{Fusion pore formation}

In previous, lipid mixing based studies performed on PSMs unstable intermediate levels in vesicle membrane fluorescence were discussed in the context of a metastable, productive hemifusion diaphragm. ${ }^{[61,85,149]}$ However, the varying levels in fluorescence intensity did not match with theoretical considerations of the fluorescence intensity time traces and were thought to possibly originate from a transient fusion pore. ${ }^{[61]}$ Indeed the results of the present study support the latter hypothesis. The amount of flickering fusion pores observed here ( $8 \%$ of the vesicle population) fit very well with the $9 \%$ of vesicles thought to fuse via a stable hemifusion intermediate. The use of content release as a direct indicator for the opening of a fusion pore used in the present study thus results in a change of the mechanistic understanding of the process of fusion pore formation. Previously, the lag time between outer and inner leaflet mixing, which was used to determine the delay of lipid mixing and fusion pore formation, was extracted from 
the lifetime of the aforementioned intermediate fusion state with half-life times of 3-4 s. ${ }^{[85,149]}$ Detecting lipid mixing and content release simultaneously with high sensitivity and time resolution allowed for a more correct extraction of the lag time between fusion pore opening and lipid diffusion over a fusion stalk. While also here lipid mixing could occur long before fusion pore formation a median lag time of only $42 \pm 11 \mathrm{~ms}$ was extracted. Notably, a large fraction of vesicles showed no lipid mixing at all concomitant with content release $(46 \%$ of fusing LUVs). This leads to the hypothesis that fusion pore formation occurs almost simultaneously with lipid mixing with a low likelihood of a stable hemifusion diaphragm. These results are in good agreement with other studies performed in cells as well as on SLBs and PSMs. ${ }^{[36,37,62]}$ For example Stratton et al. quantified a lag time of $18.2 \mathrm{~ms}$ and Takahashi et al. of $0.3 \mathrm{~s}$ (Figure 5.5) both of which used SRB as content marker. The latter larger time difference is likely caused by the limited time resolution of the setup of $0.3 \mathrm{~s}$. In this elegant study Takahashi et al. further showed that content markers of $10 \mathrm{kDa}$ or larger are delayed or in some cases hindered completely in their diffusion through the fusion pore. This finding is in agreement with a study of Lai et al. and might explain long hemifusion lifetimes reported by Zhao et al. in living cells who used a very large content marker EGFP-PH (27 kDa). ${ }^{[69,178]}$ Notably in their study only $1 / 3$ of vesicles showed this stable hemifusion diaphragm while the remaining vesicles exhibited direct full fusion behavior which is in good agreement with the present study. In conclusion, the results of the present work strongly indicate that the dominant fusion pathway induced by the minimal fusion machinery does not involve a stable hemifusion intermediate prior to fusion pore formation. ${ }^{[170]}$
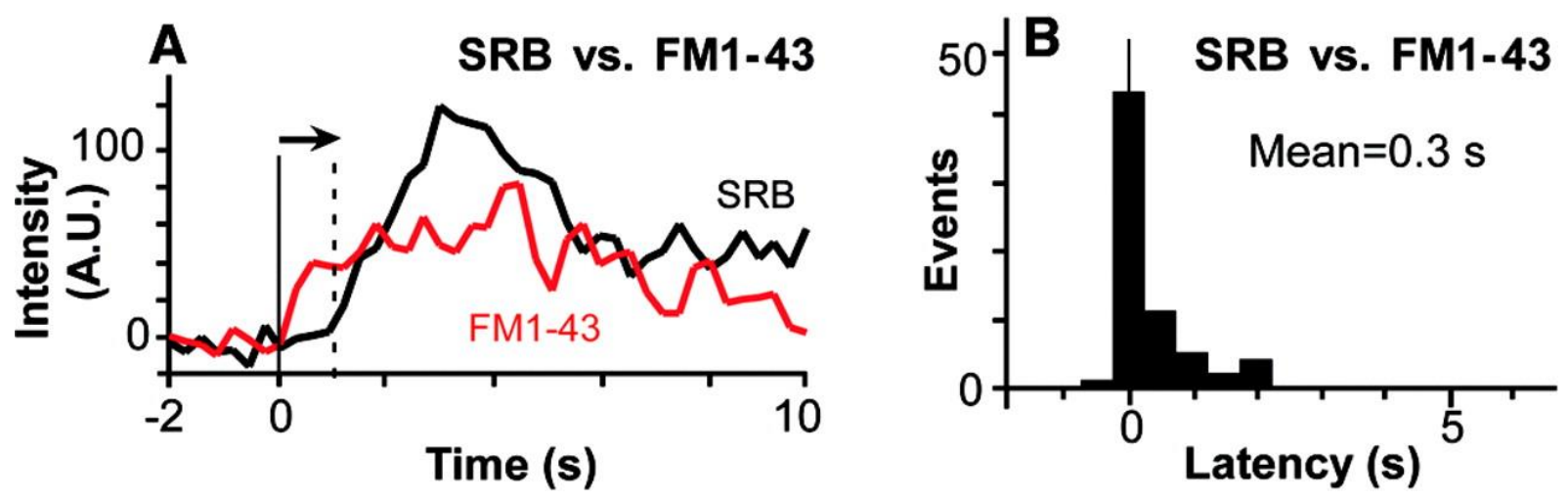

Figure 5.5 Simultaneous imaging of lipid dye (FM1-43) and content marker (SRB) influx into insulin granules. (A) Representative fluorescence intensity time trace of the lag time between lipid and content mixing with (B) the respective histogram of $N=65$ events. Figure adapted according to Takahashi et al. ${ }^{[37]}$

Next to the incorporation of the exact time point of pore opening in the fusion pathway also the kinetics of the content release process were quantified in this work. A mean half-life time of $48 \pm 7 \mathrm{~ms}$ was extracted by fitting a mono-exponential decay function to the averaged release 
profile of all 840 content release events. This mono-exponential fit indicates that the release process is similar between all individual vesicles fusing with PSMs formed on open substrates and follows a one step process. The very fast kinetic of $48 \mathrm{~ms}$ on the other hand highlights that the process of content release is little to not influenced by the fact that the vesicle is docked to the solid supported part of the PSM which makes this system a powerful tool to study the process of fusion pore formation. The same evaluation approach was conducted for vesicles fusing with PSMs formed on porous substrates with closed compartments. It revealed a release profile that could only be modeled with a bi-exponential decay function which possibly originates from a two-step release process or two underlying populations. As these measurements were one channel recordings without the use of the optosplit II, the higher signal to noise ratio enabled the extraction of each individual release profile that could be described with a mono-exponential decay function. Thus, the bi-exponential fit to the averaged data set was a result of two underlying populations. The vast majority of vesicles (83\%) released their content with half-life times $<100 \mathrm{~ms}$ very similar to the above mentioned kinetic. Possibly the slower release with half-life times of up to $700 \mathrm{~ms}$ is caused if the neighboring cavity is too small to uptake the whole vesicular content at once. The fact that the release kinetic was proven to be independent of the apparent vesicle size $(\rho=0.0981)$ indicates the existence of variations in the volume of the cavity covered by the f-PSM. This seems reasonable as spreading of GUVs on closed substrates repeatedly led to membranes adhered to the whole surface of the cavities following the gold/MH functionalization that covered the edge and the bottom of the holes (Figure $3.18 \mathrm{D}$ ). In conclusion, this leads to the hypothesis that the release kinetics are strongly dependent on the available space underneath the PSM in direct opposition to the fusion site. This further highlights the advantage of PSMs as a fusion setup compared to SLB based assays that lack this $2^{\text {nd }}$ aqueous compartment. In these assays the content release is hindered by the lack of space underneath the membrane and takes up to several seconds to reach the baseline. ${ }^{[75,79,82]}$ For example Kreutzberger et al. showed in a nice study that the half-life time of the release process and thus possibly the lifetime of the fusion pore is dependent on the concentration of PE in the target membrane with $\tau=0.3 \mathrm{~s}$ for $20 \%$ up to $\tau=1.5 \mathrm{~s}$ for $0 \%$ PE. ${ }^{[79]}$ In a similar approach, Stratton et al. reported that increasing concentrations of cholesterol also lead to a longer lifetime of the fusion pore. ${ }^{[36]}$ They reported lipid mixing kinetics of $\sim 30-250 \mathrm{~ms}$ that are similar or longer for the content release and postulate from these slow kinetics the presence of a flickering fusion pore. The latter conclusion, however, is very questionable, since the inherently slow efflux of content markers from SUVs in SBL-based model systems is most certainly caused by the narrow aqueous space underneath the membrane. 
These studies again show that kinetic observables can be used to gather meaningful information about the effects of external variables on the fusion process while using them to draw direct conclusions about the mode of fusion need to be done cautiously, as they can be influenced by the respective model system.

Nevertheless, flickering fusion pores are discussed to be an important alternative mode of fusion that is thought to activate larger amounts of postsynaptic receptors for a longer time period. ${ }^{[110]}$ They are frequently observed in vivo with frequencies between two open states ranging from 170 to $4000 \mathrm{~Hz} .{ }^{[10-114]}$ Consequently, pore flickering in the commonly used term with rapid, sub millisecond dynamics is only observed in vivo usually using amperometric recordings. However, in the present study flickering fusion was considered to be a dynamic fusion mode with two or more release events and was also reported as such by Gong et al. ${ }^{[68]}$ Both results show that vesicles can fuse dynamically with two or more consecutive pore openings in a very simplified model system using only the minimal fusion machinery of syb 2, syx 1A and SNAP25a. In their study, Gong et al. further showed that the initial docking time is significantly longer than the dwell time between the $1^{\text {st }}$ and $2^{\text {nd }}$ release event $(6.8 \pm 0.6 \mathrm{~s}$ vs. $0.21 \pm 0.01 \mathrm{~s})$ and explain this finding with a lower activation energy for the second fusion step. However, this difference in the two populations $(65 \pm 4 \mathrm{~s}$ vs. $51 \pm 10 \mathrm{~s})$ was not significant in the present work. As discussed above, the inherently large docking times observed on PSMs could make it impossible to detect the changes in activation energy.

\section{D postfusion structures}

After the formation of the fusion pore and the efflux of content molecules the vesicle had two distinct fates: it either retained its 3D structure or collapsed into the target membrane. The existence as well as lifetime of this commonly called $\Omega$-shape were deduced from the fluorescence intensity time trace of the target membrane. As described above, the influx of lipids from the PSM into the vesicular shape leads to a dequenching in fluorescence when lipids exit the quenching regime of the gold-covered pore rim. In $46 \%$ of the cases vesicle merging concomitant with content release was too rapid to detect a 3D postfusion structure while a stable $\Omega$-shape was detected in $40 \%$ of the cases. The remaining population was separated in two groups with mean overall lifetimes for the postfusion structure of $50 \pm 5 \mathrm{~s}$ and $10 \pm 1 \mathrm{~s}$. The total lifetime of the 3D postfusion structure was further divided in the time until the onset of vesicle collapse $t_{\text {rise }}$ and the collapse process $t_{\text {collapse. }}$ Expectedly, the maximum level of lipid dye fluorescence during $t_{\text {rise }}$ strongly correlated with the apparent vesicle size $(\rho=0.6022)$. However, neither $t_{\text {rise }}, t_{\text {collapse }}$ or $t_{\text {rise+collapse }}$ were influenced significantly by the LUV size. In 
addition, a fast rise time did not correlate with a fast collapse time and vice versa. This raises the question what drives the fusing vesicles to behave so differently in their 3D postfusion structure. The collapse kinetics observed in this work ( $\tau_{\text {collapse }}$ of $23 \pm 1 \mathrm{~s}, 46 \%$ and $2.4 \pm 0.1 \mathrm{~s}$, $54 \%)$ are in good agreement with previous results obtained on PSMs ${ }^{[85,86,149]}$ as well as those observed for insulin granule vesicles. ${ }^{[37]}$ In the latter study Takahashi et al. extracted average

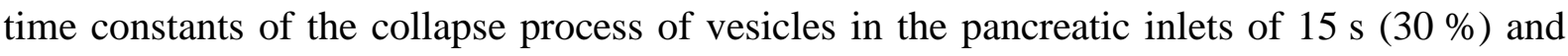
$1.5 \mathrm{~s}(70 \%)$. Previously, the existence of such two populations was also reported by Kuhlmann in a lipid mixing based fusion setup on PSMs. ${ }^{[61]} \mathrm{He}$ correlated longer collapse times with a possibly elongated release kinetic of content molecules due to hindering effects of the underlying substrate. However, the present work shows that the process of content release is unhindered and uniform on porous substrates with open cavities and that the obtained half-life times are 2-3 orders of magnitude smaller than those of the $\Omega$-shape. Alternatively, local differences in membrane tension could induce a fast collapse of the vesicle as shown by in vivo studies that report for a connection between membrane tension and vesicle merging. ${ }^{[179,180]}$ Another explanation is given by in vivo studies of the fusion process of secretory granules that show similar complex and long lived 3D postfusion structures. ${ }^{[109,177,180]}$ These stable postfusion structures can be discussed in the context of kiss-and-run exocytosis (KR) as an alternative fusion pathway to direct full fusion. It is thought that a longer stable 3D postfusion structure would enable fast endocytosis $\left(\sim 1 \mathrm{~s}^{-1}\right)$ and thus the preservation of vesicular shape, its proteins, and even lipids. ${ }^{[182-184]}$ For example, by using super-resolution fluorescence microscopy Chiang et al. directly visualized $\Omega$-profiles of dense-core vesicles (DCVs). The observed $\Omega$-shapes were classified in 7 different modes with the vesicle either enlarging, retaining its size, shrinking completely or incompletely, and the fusion pore staying open or closed. A large fraction of vesicles (49\%) showed a re-closing of the fusion pore which directly correlated with rapid endocytosis. Notably, these percentages are related to the whole vesicle population and vesicle fusion without visible lipid mixing, in contrast to the present study, was not reported in their work. However, when replacing synthetic vesicles with natural chromaffine granules and monitoring their fusion behavior on PSMs, an $\Omega$-shape is present for $100 \%$ of the fusing vesicles with only $1 \%$ merging completely into the target membrane. ${ }^{[87]}$ This finding is in contrast to the observation that an increase in vesicle size might lead to a re-closure of the fusion pore prior to complete release and consequently a stable $\Omega$-shape, as the mean diameter of CGs is with $167 \pm 14.3 \mathrm{~nm}$ slightly lower than the diameter of LUVs used in this work. ${ }^{[185]}$ 
However, differences in the lipid mixture of CGs and its protein-dense membrane might have an influence on the lifetime of the fusion pore as well.

In summary, on the one hand a stable $\Omega$-shape concomitant with the re-closing of the fusion pore can be associated with KR fusion. On the other hand, a preferred full content release via a rapidly collapsing or non-existing 3D postfusion structure show distinctive features of the classical full-collapse fusion mode observed in vivo. The results of this work indicate that these fusion modes that induced by a very simplified fusion machinery might be influenced by the vesicle size and the lifetime of the fusion pore. It is, however, plausible that the underlying mechanisms that determines whether a vesicle fuses via direct full fusion or KR involves several more factors. Differences in lipid compositions, a more densely packed membrane, and larger content molecules most likely influence the fusion pathway and content release kinetics. ${ }^{[36,37,79,82]}$ In addition, factors such as $\mathrm{Ca}^{2+}$ concentration and regulatory proteins are commonly discussed to influence the mode of fusion as well. ${ }^{[38,109]}$ Nevertheless, the results of the present work strongly suggest that the complexity of the fusion process observed in vivo is an intrinsic feature of the minimal fusion machinery and that the straight forward fusion pathway introduced in Figure 1.3 needs to be re-defined. In conclusion, the convolution of important fusion pathways in correlation with quantified key kinetic observables leads to the fusion model proposed in Figure 5.6.

Thus, this study sets the groundwork to investigate the influence of different factors such as lipid composition and vesicle-surface interaction on the fusion pathway, mode of fusion, and important kinetics. 


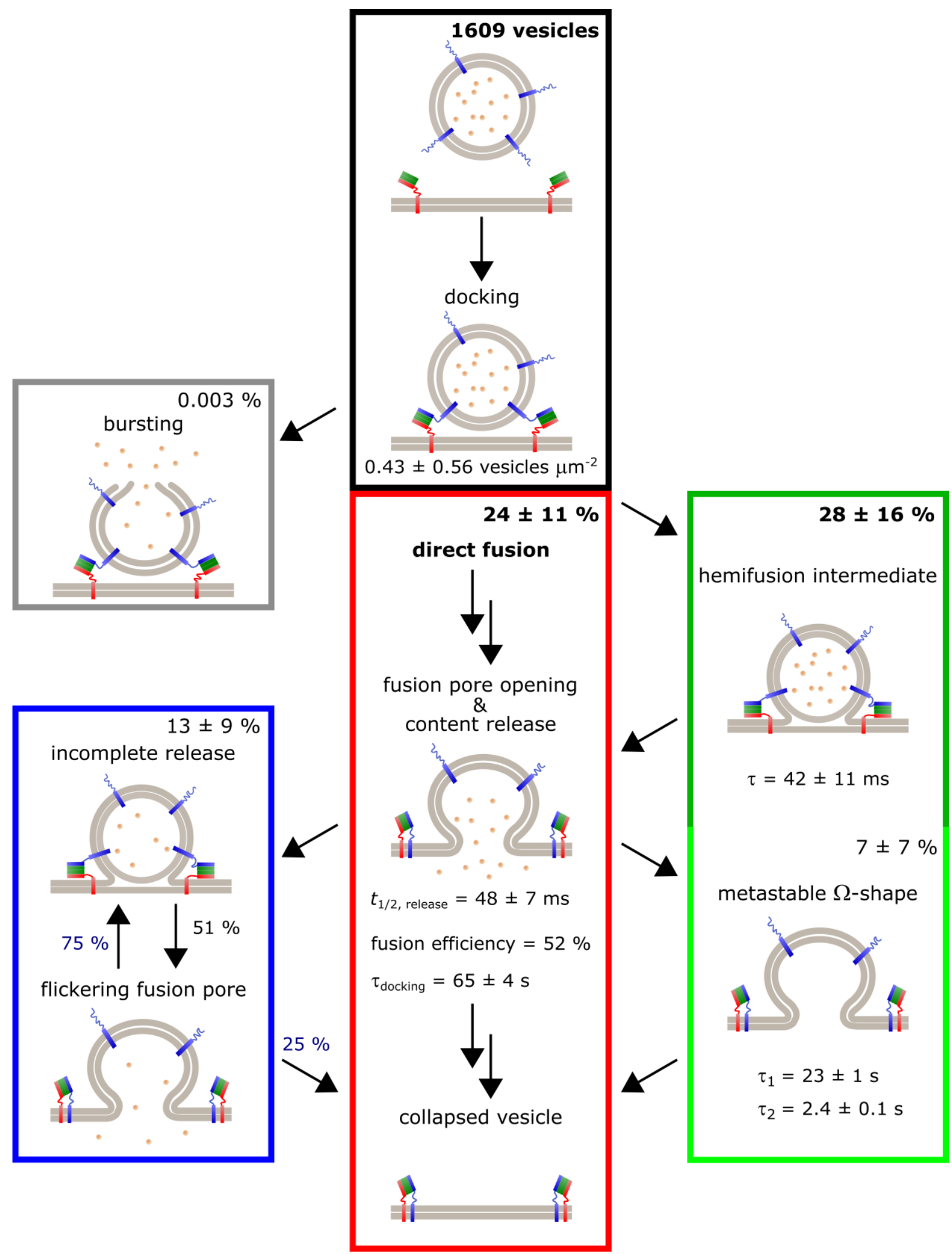

Figure 5.6 Summary of important fusion pathways, their statistics, and key kinetic observables determined for the process of SNARE-mediated membrane fusion of syb 2 doped proteo-LUVs (DOPC/POPE/POPS/cholesterol; $5 / 2 / 1 / 2(n / n), 43 \mathrm{mM} \mathrm{SRB}, d=240 \pm 100 \mathrm{~nm}$, effective p/l 1:600) with t-SNARE acceptor complex $\Delta \mathrm{N} 49$ containing PSMs (DOPC/POPE/POPS/cholesterol/Atto655 DPPE; 5/1.9/1/2/0.1 ( $n / n$ ), effective p/l 1:1900). 


\section{Summary and Conclusion}

The opening of an exocytotic fusion pore and the concomitant transfer of neurotransmitter over the plasma membrane into the synaptic cleft is the key step during neuronal signal transmission and is driven by SNARE proteins. The goal of this thesis was to establish an in vitro model system capable of visualizing this crucial step of content transfer to gather meaningful insights in the process of fusion pore formation and other fusion intermediates to thus analyze possible fusion pathways and quantify important kinetics.

To this end, the minimal fusion machinery comprising of synaptobrevin 2 (syb 2) and the fusion active acceptor complex $\Delta \mathrm{N} 49$ were functionally reconstituted into sulforhodamine $\mathrm{B}$ (SRB) filled large unilamellar vesicles (LUVs) and lipid labeled pore-spanning membranes (PSMs), respectively. PSMs are planar continuous lipid bilayers that are formed on a highly porous substrate and thus contain both solid supported parts (s-PSM) as well as freestanding membranes (f-PSM) and are easily accessible via fluorescence microscopy. While the solid supported part leads to their long term stability, f-PSMs that span over large aqueous compartments provide enough space for an unhindered content transfer to occur. Thus, by using high speed fluorescence microscopy the content transfer of SRB across the target membrane through a SNARE-mediated fusion pore was directly visualized on a single-vesicle level. Simultaneous detection of the diffusion of lipid labels from the PSM into the membrane of the fusing vesicle enabled to monitor and quantify different fusion pathways. Subsequently, combining insights of these two readout parameters was used to gather information about the interplay of fusion pore opening and lipid mixing.

Analyzing the docking behavior of > 1600 proteo-LUVs to 68 individual PSMs revealed a large variation in docking efficiencies of $0.43 \pm 0.56$ docked vesicles per $\mu \mathrm{m}^{2}$ membrane. As the reconstitution efficiency of the $\Delta \mathrm{N} 49$-complex into GUVs was quantified to be $26 \pm 24 \%$, this variance most likely originated from differences in acceptor complex densities inside the PSM. Yet, neither the fusion efficiency nor the docking time of vesicle $\left(t_{\text {docking }}\right)$ was influenced by these variations resulting in an overall fusion efficiency of $52 \%$ and an average docking lifetime of $\tau_{\text {docking }}=65 \pm 4 \mathrm{~s}$. The fact that vesicles preferably docked at the edges of the s-PSM, partially caused by larger protein concentration at this part of the PSM, enabled the direct visualization of content transfer through a fusion pore across the PSM into the $2^{\text {nd }}$ aqueous compartment. Additionally, these events could be differentiated from the rarely $(0.003 \%)$ occurring bursting of vesicles. Furthermore, fusing vesicles exhibited rapid and uniform content release kinetics with a half-life time of the release process of $48 \pm 7 \mathrm{~ms}$. Performing the same 
evaluation for the fusion of vesicles with PSMs that span very small, confined aqueous compartments suggest that unhindered content release is enabled by a large aqueous space underneath the f-PSM in direct vicinity of the fusion site.

Of all vesicles, $24 \pm 11 \%$ fused without detectable lipid mixing and collapsed instantaneously into the target membrane, while $28 \pm 16 \%$ fused via a hemifusion intermediate. The lifetime of this state, defined as the lag time between onset of lipid mixing and content release, was determined to be only $42 \pm 11 \mathrm{~ms}$. Thus, the short lifetime or non-existence of a hemifusion intermediate indicates that the two processes occur almost simultaneously and the preferred fusion pathway does not include a long lived hemifusion intermediate. Additionally, in conjunction with immediate merging of $24 \pm 11 \%$ of vesicles into the PSM, a significant amount of vesicles showed a fast collapse $(\tau=2.4 \pm 0.1 \mathrm{~s})$ into the membrane. Consequently, the preferred fusion pathway mediated by the minimal fusion machinery shows distinctive features of the fusion mode called full-collapse fusion often observed in vivo. However, the 3D structure of the vesicle, also known as $\Omega$-shape, remained intact for $21 \%$ of all events, probably due to a reclosing of the fusion pore which was more likely for larger vesicles. This fusion behavior shows distinct features of kiss-and-run exocytosis observed in vivo during which the synaptic vesicle retains its shape after fusion and is directly retrieved from the presynaptic membrane. Most of the time this closing of the fusion pore is accompanied with an incomplete content release from which the fusion pore can re-open with a $51 \%$ likelihood. This flickering fusion pore leads to an incomplete release in $75 \%$ of events. Thus, in total $13 \pm 9 \%$ of vesicles released their content incompletely as the final state. The observation of these numerous fusion pathways leads to the hypothesis that the complexity of the fusion behavior observed in vivo is already present in a fusion process catalyzed by the minimal fusion machinery. While the fusion modes proved to be independent of different acceptor complex densities they might be influenced by the lifetime of the fusion pore and the size of the vesicle.

The results of this work thus allow for future studies to investigate the influence of different parameters such as lipid composition, vesicle size, and regulatory proteins on the modes of fusion and key kinetic parameters in a well-defined environment. Alternatively, the introduction of PEG linkers to the membrane of SRB filled proteo-liposomes, thus imitating the densely packed environment of membranes in vivo, could enable their unhindered diffusion on both the f-PSM and s-PSM. Their fusion behavior could then be compared with that of content labeled natural vesicles in a model system that recreates important physiological parameters. 


\section{Appendix}

\subsection{Amino acid sequences of SNAREs}

Synaptobrevin 2 (aa 1-116)

10

20

30

40

MSATAATVPP AAPAGEGGPP APPPNLTSNR RLQQTQAQVD

50

60

70

80

EVVDIMRVNV

DKVLERDQKL

SELDDRADAL

QAGASQFETS

90

100

110

AAKLKRKYWW

KNLKMMIILG

VICAIILIII

IVYFST

Syntaxin 1A (aa 183-288)

190

200

210

220

MDSSISKQ

ALSEIETRHS

EIIKLENSIR ELHDMFMDMA

230

240

250

260

MLVESQGEMI

DRIEYNVEHA

VDYVERAVSD TKKAVKYQSK

270

280

ARRKKIMIII

CCVILGIIIA

STIGGIFG

SNAP25a (aa 1-206)

10

20

30

40

MAEDADMRNE LEEMQRRADQ

LADESLESTR

RMLQLVEESK

50

60

70

80

DAGIRTLVML DEQGEQLERI

EEGMDQINKD MKEAEKNLTD

90

100

110

120

LGKSSGLFIS

130

140

150

160

VDEREQMAIS

GGFIRRVTND

ARENEMDENL

EQVSGIIGNL

170

180

190

200

RHMALDMGNE

IDTQNRQIDR

IMEKADSNKT

RIDEANQRAT

KMLGSG 


\subsection{List of symbols and abbreviations}

3D

A

aa

APS

$\mathrm{au}$

$B G$

C

CF

CGs

CLSM

$\mathrm{cm}$

$d$

$D$

DCVs

$D O L$

DOPC

DPPE

dsGUVs

DTT

E. coli

e.g.

EMCCD

F

FCS

fps

f-PSM

FRAP

FRET

GUV

$\mathrm{h}$ three dimensional

area

amino acid

ammonium persulfate

arbituary unit

background

cysteine

correction factor

chromaffine granules

confocal laser scanning microscopy

centimeter

diameter, thickness

diffusion coefficient

dense-core vesicles

degree of labeling

1,2-dioleoyl-sn-glycero-3-phosphocholine

1,2-dipalmitoyl-sn-glycero-3-phosphoethanolamine

droplet stabilized GUVs

dithiothreitol

Escheria choli

exempli gratia

electron multiplying charged coupled device

fluorescence intensity

fluorescence correlation spectroscopy

frames per seconds

freestanding part of the PSM

fluorescence recovery after photobleaching

fluorescence resonance energy transfer

giant unilamellar vesicle

hour 
HEPES 4-(2-hydroxyethyl)-1-piperazineethanesulfonic acid

$\mathrm{Hz} \quad$ Hertz

IEC ion exchange chromatography

$I_{\mathrm{P}} \quad$ protein concentration

IPTG isopropyl- $\beta$-d-thiogalactopyranoside

IQR interquartile range

ITO indium tin oxide

$k \quad$ rate constant

$\mathrm{kDa} \quad$ kilodalton

KR kiss-and-run

L liter

LB-medium lysogeny broth medium

LUV large unilamellar vesicle

M molar

$\mathrm{mg} \quad$ milligram

MH 6-mercapto-1-hexanol

$\min \quad$ minute

$\mathrm{mL} \quad$ milliliter

mOsM milliosmolar

$M_{\text {ref }} \quad$ reference slope

MSD mean squared displacement

$\mathrm{mW} \quad$ milliwat

NA numeric aperture

NBD-PE N-(7-nitro-2,1,3-benzoxadiazole-4-yl)-phosphatidylethanolamine

NEM N-ethylmaleimide

nm nanometer

NSF $\quad N$-ethylmaleimide-sensitive factor

$O D_{600} \quad$ optical thickness at $600 \mathrm{~nm}$

$\mathrm{p} / 1 \quad$ protein to lipid ratio

PEG poly ethylene glycol

pI isoelectric point

POPE 1-palmitoyl-2-oleoyl-sn-glycero-3-phosphoethanolamine 


\begin{tabular}{|c|c|}
\hline POPS & 1-palmitoyl-2-oleoyl-sn-glycero-3-phospho-L-serine \\
\hline PSMs & pore-spanning membranes \\
\hline Q & glutamine \\
\hline $\mathrm{R}$ & arginine \\
\hline$R$ & reconstitution efficiency \\
\hline$r_{e}$ & effective bleach radius \\
\hline $\mathrm{Rh}-\mathrm{PE}$ & $N$-(lissamine rhodamine B sulfonyl)-phosphatidylethanolamine \\
\hline$r_{n}$ & nominal bleach radius \\
\hline ROI & region of interest \\
\hline $\mathrm{rpm}$ & revolutions per minute \\
\hline RT & room temperature \\
\hline s & second \\
\hline $\mathrm{S}$ & serine \\
\hline SAM & self-assembled monolayer \\
\hline$S D$ & standard deviation \\
\hline SDCM & spinning disc confocal microscopy \\
\hline SDS-PAGE & sodium dodecyl sulfate polyacrylamide gel electrophoresis \\
\hline SLB & supported lipid bilayer \\
\hline SNAP & soluble NSF attachment protein \\
\hline SNAP25 & synaptosomal associated protein of $25 \mathrm{kDa}$ \\
\hline SNAREs & soluble $N$-ethylmaleimide-sensitive factor attachment receptors \\
\hline s-PSM & solid supported part of the PSM \\
\hline SPT & single particle tracking \\
\hline SRB & sulforhodamine B (acid chloride) \\
\hline SUV & small unilamellar vesicle \\
\hline syb 2 & synaptobrevin 2 \\
\hline syx $1 \mathrm{~A}$ & syntaxin $1 \mathrm{~A}$ \\
\hline$t_{1 / 2}$ & half-life time \\
\hline TCEP & tris(2-carboxyethyl)phosphine \\
\hline$t_{\text {docking }}$ & docking time \\
\hline TEMED & tetramethylethlenediamine \\
\hline TIRF & total internal reflection fluorescence \\
\hline
\end{tabular}




$\begin{array}{ll}\text { TMD } & \text { transmembrane domain } \\ \text { TRIS } & \text { tris(hydroxymethyl)aminomethane } \\ \text { t-SNARE } & \text { target SNARE } \\ \text { UV/Vis } & \text { Ultraviolet/visible } \\ \text { V } & \text { volt } \\ v / v & \text { volume per volume } \\ \text { v-SNARE } & \text { vesicular SNARE } \\ w / v & \text { weight per volume } \\ w_{\mathrm{i}} & \text { statistical weighting factor } \\ \Delta t & \text { time difference } \\ \varepsilon & \text { exctinction coefficient } \\ \lambda_{\mathrm{abs}} & \text { absorption wavelength } \\ \lambda_{\mathrm{em}} & \text { emission wavelength } \\ \lambda_{\mathrm{ex}} & \text { excitation wavelength } \\ \mu \mathrm{m} & \text { micrometer } \\ \rho & \text { correlation coefficient } \\ \tau & \text { life time } \\ \tau_{1 / 2} & \text { half time of fluorescence recovery }\end{array}$

\subsection{List of chemicals and consumables}

\section{Chemicals}

6-Mercapto-1-hexanol

APS

Argon

Atto488-Maleimide

Atto655/488/390-DPPE

CHAPS

Chloroform

Cholesterol

Coomassie G-250

DOPC
Sigma-Aldrich (Taufkirchen, GER)

Sigma-Aldrich (Taufkirchen, GER)

Linde (München, GER)

Atto-tec (Siegen, GER)

Atto-tec (Siegen, GER)

Carl Roth GmbH (Karlsruhe, GER)

Merck (Darmstadt, GER)

Sigma-Aldrich (Taufkirchen, GER)

Fisher Scientific GmbH (Schwerte, GER)

Avanti Polar Lipids (Alabaster, USA) 
DTT

EDTA

Ethanol

Glucose

Glycerin

Gold, $99.99 \%$

$\mathrm{HCl}$

HEPES

Imidazole

Kanamycinsulfat

$\mathrm{KCl}$

$\mathrm{KOH}$

$\mathrm{NaCl}$

$\mathrm{NaOH}$

$n-\mathrm{OG}$

n-propanole

POPE

POPS

Rotiphorese $\mathrm{Gel}^{\mathrm{TM}}$

SDS

Sephadex ${ }^{\mathrm{TM}} \mathrm{G}-15$

Sucrose

Sulforhodamine B acid chloride

TCEP

TEMED

Thrombine

Titan

TRIS

Tryptone

Urea

Yeast extract
Carl Roth GmbH (Karlsruhe, GER)

Carl Roth GmbH (Karlsruhe, GER)

Sigma-Aldrich (Taufkirchen, GER)

Sigma-Aldrich (Taufkirchen, GER)

Grüssing GmbH (Filsum, GER)

Allgemeine Gold- und Silberscheideanstalt (Pforzheim, GER)

VWR International (Darmstadt, GER)

Carl Roth GmbH (Karlsruhe, GER)

Sigma-Aldrich (Taufkirchen, GER)

Carl Roth GmbH (Karlsruhe, GER)

Fisher Scientific GmbH (Schwerte, GER)

Grüssing GmbH (Filsum, GER)

Carl Roth GmbH (Karlsruhe, GER)

Mallinckrodt Baker B.V. (Deventer, NED)

Carl Roth GmbH (Karlsruhe, GER)

Carl Roth GmbH (Karlsruhe, GER)

Avanti Polar Lipids (Alabaster, USA)

Avanti Polar Lipids (Alabaster, USA)

Carl Roth GmbH (Karlsruhe, GER)

AppliChem (Darmstadt, GER)

GE-Healthcare (Chalfont, UK)

Acros Organics (New Jersey, USA)

Sigma-Aldrich (Taufkirchen, GER)

Carl Roth GmbH (Karlsruhe, GER)

Sigma-Aldrich (Taufkirchen, GER)

Sigma-Aldrich (Taufkirchen, GER)

Elektronen Optik Service GmbH (Dortmund)

Carl Roth GmbH (Karlsruhe, GER)

Carl Roth GmbH (Karlsruhe, GER)

Merck KGaA (Darmstadt, GER)

Carl Roth GmbH (Karlsruhe, GER) 


\section{Consumables}

Copperband

Eco-column, $3 \times 13$ centimeter

ITO-Slides

MonoQ/S column

NAP $^{\mathrm{TM}}-10 \& 25$ Column, G-25

Polycarbonatemembrane, $400 \mathrm{~nm}$

Porous substrates $(d=1.2 \& 5.0 \mu \mathrm{m})$

Porous substrates $(d=3.5 \mu \mathrm{m}, \mathrm{h}=0.25 \mu \mathrm{m})$
Präzisions Glas \& Optik GmbH (Iserlohn)

BioRad Laboratory (Berkeley, USA)

Präzisions Glas \& Optik GmbH (Iserlohn)

GE Healthcare (Little Chalfont, UK)

GE Healthcare (Little Chalfont, UK)

Avestin (Ontario, Canada)

Aquamarijn (Zutphen, The Netherlands)

caesar (Bonn, GER)

\subsection{List of devices and software}

\section{Surface coating}

Zepto plasma cleaner

Sputtercoater 108 auto

Coating System MED020

Fluorescence spectroscopy

JASCO FP 6500

\section{UV/Vis-spectroscopy}

Varian Cary Scan 50

NanoDrop200c
Diener Electronics (Ebbhausen, GER)

Cressington Scientific Instruments (Watford, UK)

Bal-Tec (Balzers, Lichtenstein)

JASCO (Easton, USA)

Thermo Fisher Scientific (Waltham, USA)

EPI fluorescence microscopy

BX 51

Olympus (Tokyo, Japan)

objective: ACHRORPLAN 40Xw, NA 0.8 Carl Zeiss Microscopy (Thornwood, USA)

filter: U-MNB2

Olympus (Tokyo, Japan)

camera: iXon EMCCD

Andor Technology (Belfast, UK)

mercury vapor lamp: U-HGLPS

Andor Technology (Belfast, UK)

\section{Confocal laser scanning microscopy (CLSM)}

FluoView 1200

objective: LUMFLN 60Xw, NA 1.1
Olympus (Tokyo, Japan)

Olympus (Tokyo, Japan) 


\section{Spinning disc confocal microscopy (SDCM)}

spinning disc: Yokogawa CSU-X

stand: custom, based on ix73

AOTF: TF525-250-6-3-GH18A

objective: LUMFLN 60Xw, NA 1.1

laser: iBeam Smart 488-S

laser: iBeam Smart 561-S

laser: iBeam Smart 639-S

camera: iXON 897 Ultra

filter: ZET405/488/561/640

single channel mode

filter: 590 LP ET longpass

filter: 525/50 ET bandpass

\section{two channel mode}

optosplit II

filter: 595/40 ET bandpass

filter: 655 LP ET longpass

H 643 LPXR superflat beamsplitter

\section{Miscelaneous}

ÄKTA purification system

Concentrator 5301

frequency generator: Aglient 333220A

Galaxy mini

LiposoFast $^{\circledR}$

Micro scale: CP225D

MilliQ Gradient A10

Osmomat 030

pH meter: Calimatic 766

thermomixer compact

microfluidizer LM10
Rota Yokogawa GmbH \& Co. KG (Wehr, GER)

Olympus (Tokyo, Japan)

Gooch \& Housego PLC (Ilminster, UK)

Olympus (Tokyo, Japan)

Toptica Photonics AG (Gräflingen, GER)

Toptica Photonics AG (Gräflingen, GER)

Toptica Photonics AG (Gräflingen, GER)

Andor Technology Ltd. (Belfast, UK)

AHF Analysentechnik AG (Tübingen, GER)

AHF Analysentechnik AG (Tübingen, GER)

AHF Analysentechnik AG (Tübingen, GER)

Acal BFi Germany (Dietzenbach, GER)

AHF Analysentechnik AG (Tübingen, GER)

AHF Analysentechnik AG (Tübingen, GER)

AHF Analysetechnik AG (Tübingen, GER)

GE Healthcare (Little Chalfont, UK)

Eppendorf (Hamburg, GER)

Aglient Technology (Santa Clara, USA)

VWR International (Darmstadt, GER)

Avestin (Ontario, CAN)

Satorius (Göttingen, GER)

Millipore (Eschborn, GER)

Gonotec (Berlin, GER)

Knick (Berlin, GER)

Eppendorf (Hamburg, GER)

Microfluidics Corp. (MA, USA)

\section{Software}


ImageJ $1.52 \mathrm{t}$

IQ 2.9

FluoView 1200

Matlab R2019b

OriginPro $8.5 \mathrm{G}$

Solis htttp://imagej.nih.gov/ij

Andor Technology Ltd. (Belfast, UK)

Olympus (Tokyo, Japan)

Math Works (Natick, USA)

OriginLab Corporation (Northampton, USA)

Andor Technology Ltd. (Belfast, UK) 


\section{Bibliography}

1. Block, M. R. et al., Purification of an N-ethylmaleimide-sensitive protein catalyzing vesicular transport. Proc. Natl. Acad. Sci. USA 1988, 85: 7852-7856.

2. Malhotra, V. et al., Role of an N-ethylmaleimide-sensitive transport component in promoting fusion of transport vesicles with cisternae of the Golgi stack. Cell 1988, 54: 221-227.

3. Clary, D. O., I. C. Griff, and J. E. Rothman, SNAPs, a family of NSF attachment proteins involved in intracellular membrane fusion in animals and yeast. Cell 1990, 61: 709-721.

4. Söllner, T. et al., SNAP receptors implicated in vesicle targeting and fusion. Nature 1993, 362: 318-324.

5. Jahn, R., and T. C. Südhof, Membrane fusion and exocytosis. Annu. Rev. Biochem. 1999, 68: 863-911.

6. Munson, M., Synaptic-vesicle fusion: a need for speed. Nat. Struct. Mol. Biol. 2015, 22: $509-511$.

7. Sudhof, T. C., The synaptic vesicle cycle. Annu. Rev. Neurosci. 2004, 27: 509-547.

8. Jahn, R., and D. Fasshauer, Molecular machines governing exocytosis of synaptic vesicles. Nature 2012, 490: 201-207.

9. Südhof, T. C., Neurotransmitter release. The last millisecond in the life of a synaptic vesicle. Neuron 2013, 80: 675-690.

10. Weber, T. et al., SNAREpins. Minimal Machinery for Membrane Fusion. Cell 1998, 92: $759-772$.

11. Fernandez, I. et al., Three-Dimensional Structure of an Evolutionarily Conserved NTerminal Domain of Syntaxin 1A. Cell 1998, 94: 841-849.

12. Fasshauer, D. et al., A structural change occurs upon binding of syntaxin to SNAP-25. J. Biol. Chem. 1997, 272: 4582-4590.

13. Fasshauer, D. et al., Structural changes are associated with soluble N-ethylmaleimidesensitive fusion protein attachment protein receptor complex formation. J. Biol. Chem. 1997, 272: 28036-28041.

14. Fasshauer, D., W. Antonin, V. Subramaniam, and R. Jahn, SNARE assembly and disassembly exhibit a pronounced hysteresis. Nat. Struct. Biol. 2002, 9: 144-151.

15. Sutton, R. B., D. Fasshauer, R. Jahn, and A. T. Brunger, Crystal structure of a SNARE complex involved in synaptic exocytosis at 2.4 A resolution. Nature 1998, 395: 347-353. 
16. Fasshauer, D., R. B. Sutton, A. T. Brunger, and R. Jahn, Conserved structural features of the synaptic fusion complex: SNARE proteins reclassified as Q- and R-SNAREs. Proc. Natl. Acad. Sci. USA 1998, 95: 15781-15786.

17. Weimbs, T. et al., A conserved domain is present in different families of vesicular fusion proteins: a new superfamily. Proc. Natl. Acad. Sci. USA 1997, 94: 3046-3051.

18. Kloepper, T. H., C. N. Kienle, and D. Fasshauer, An elaborate classification of SNARE proteins sheds light on the conservation of the eukaryotic endomembrane system. Mol. Biol. Cell 2007, 18: 3463-3471.

19. Hanson, P. I., J. E. Heuser, and R. Jahn, Neurotransmitter release - four years of SNARE complexes. Curr. Opin. Neurobiol. 1997, 7: 310-315.

20. Stein, A., G. Weber, M. C. Wahl, and R. Jahn, Helical extension of the neuronal SNARE complex into the membrane. Nature 2009, 460: 525-528.

21. van den Bogaart, G., T. Lang, and R. Jahn, Microdomains of SNARE proteins in the plasma membrane. Curr. Top. Membr. 2013, 72: 193-230.

22. Li, F. et al., Energetics and dynamics of SNAREpin folding across lipid bilayers. Nat. Struct. Mol. Biol. 2007, 14: 890-896.

23. Liang, B., V. Kiessling, and L. K. Tamm, Prefusion structure of syntaxin-1A suggests pathway for folding into neuronal trans-SNARE complex fusion intermediate. Proc. Natl. Acad. Sci. USA 2013, 110: 19384-19389.

24. Ellena, J. F. et al., Dynamic structure of lipid-bound synaptobrevin suggests a nucleationpropagation mechanism for trans-SNARE complex formation. Proc. Natl. Acad. Sci. USA 2009, 106: 20306-20311.

25. Álvarez de Toledo, G., M. Á. Montes, P. Montenegro, and R. Borges, Phases of the exocytotic fusion pore. FEBS Letters 2018, 592: 3532-3541.

26. LoGiudice, L., and G. Matthews, The synaptic vesicle cycle: is kissing overrated? Neuron 2006, 51: 676-677.

27. Han, X. et al., Transmembrane Segments of Syntaxin Line the Fusion Pore of Ca2+Triggered Exocytosis. Science 2004, 304: 289-292.

28. van den Bogaart, G. et al., One SNARE complex is sufficient for membrane fusion. Nat. Struct. Mol. Biol. 2010, 17: 358-364.

29. Shi, L. et al., SNARE proteins: one to fuse and three to keep the nascent fusion pore open. Science 2012, 335: 1355-1359.

30. Hernandez, J. M. et al., Variable cooperativity in SNARE-mediated membrane fusion. Proc. Natl. Acad. Sci. USA 2014, 111: 12037-12042. 
31. Risselada, H. J., C. Kutzner, and H. Grubmüller, Caught in the act: visualization of SNARE-mediated fusion events in molecular detail. ChemBioChem 2011, 12: 1049-1055.

32. Risselada, H. J., and H. Grubmüller, How SNARE molecules mediate membrane fusion: recent insights from molecular simulations. Curr. Opin. Struct. Biol. 2012, 22: 187-196.

33. Kweon, D.-H., B. Kong, and Y.-K. Shin, Hemifusion in Synaptic Vesicle Cycle. Front. Mol. Neurosci. 2017, 10: 65.

34. Chernomordik, L. V., and M. M. Kozlov, Membrane hemifusion: crossing a chasm in two leaps. Cell 2005, 123: 375-382.

35. Xu, Y. et al., Hemifusion in SNARE-mediated membrane fusion. Nat. Struct. Mol. Biol. 2005, 12.

36. Stratton, B. S. et al., Cholesterol Increases the Openness of SNARE-Mediated Flickering Fusion Pores. Biophys. J. 2016, 110: 1538-1550.

37. Takahashi, N. et al., Fusion pore dynamics and insulin granule exocytosis in the pancreatic islet. Science 2002, 297: 1349-1352.

38. Alabi, A. A., and R. W. Tsien, Perspectives on kiss-and-run. Role in exocytosis, endocytosis, and neurotransmission. Annu. Rev. Physiol. 2013, 75: 393-422.

39. Harata, N. C. et al., Frequency-dependent kinetics and prevalence of kiss-and-run and reuse at hippocampal synapses studied with novel quenching methods. Neuron 2006, 49: 243-256.

40. Sun, J.-Y., X.-S. Wu, and L.-G. Wu, Single and multiple vesicle fusion induce different rates of endocytosis at a central synapse. Nature 2002, 417: 555-559.

41. He, L., and L.-G. Wu, The debate on the kiss-and-run fusion at synapses. Trends Neurosci. 2007, 30: 447-455.

42. Brunger, A. T., D. J. Cipriano, and J. Diao, Towards reconstitution of membrane fusion mediated by SNAREs and other synaptic proteins. Crit. Rev. Biochem. Mol. Biol. 2015, 50: 231-241.

43. Hanson, P. I., H. Otto, N. Barton, and R. Jahn, The N-ethylmaleimide-sensitive fusion protein and alpha-SNAP induce a conformational change in syntaxin. J. Biol. Chem. 1995, 270: 16955-16961.

44. Lerman, J. C., J. Robblee, R. Fairman, and F. M. Hughson, Structural analysis of the neuronal SNARE protein syntaxin-1A. Biochemistry 2000, 39: 8470-8479.

45. Dulubova, I. et al., A conformational switch in syntaxin during exocytosis: role of munc18. EMBO J. 1999, 18: 4372-4382. 
46. Margittai, M. et al., Single-molecule fluorescence resonance energy transfer reveals a dynamic equilibrium between closed and open conformations of syntaxin 1. Proc. Natl. Acad. Sci. USA 2003, 100: 15516-15521.

47. Parlati, F. et al., Rapid and efficient fusion of phospholipid vesicles by the alpha-helical core of a SNARE complex in the absence of an N-terminal regulatory domain. Proc. Natl. Acad. Sci. USA 1999, 96: 12565-12570.

48. Fix, M. et al., Imaging single membrane fusion events mediated by SNARE proteins. Proc. Natl. Acad. Sci. USA 2004, 101: 7311-7316.

49. Gandasi, N. R., and S. Barg, Contact-induced clustering of syntaxin and munc18 docks secretory granules at the exocytosis site. Nat. Commun. 2014, 5: 3914.

50. Fasshauer, D., and M. Margittai, A transient N-terminal interaction of SNAP-25 and syntaxin nucleates SNARE assembly. J. Biol. Chem. 2004, 279: 7613-7621.

51. Pobbati, A. V., A. Stein, and D. Fasshauer, N- to C-Terminal SNARE Complex Assembly Promotes Rapid Membrane Fusion. Science 2006, 313: 673-676.

52. Li, F., N. Tiwari, J. E. Rothman, and F. Pincet, Kinetic barriers to SNAREpin assembly in the regulation of membrane docking/priming and fusion. Proc. Natl. Acad. Sci. USA 2016, 113: 10536-10541.

53. Kreutzberger, A. J. B., B. Liang, V. Kiessling, and L. K. Tamm, Assembly and Comparison of Plasma Membrane SNARE Acceptor Complexes. Biophys. J. 2016, 110: 2147-2150.

54. Struck, D. K., D. Hoekstra, and R. E. Pagano, Use of resonance energy transfer to monitor membrane fusion. Biochemistry 1981, 20: 4093-4099.

55. McIntyre, J. C., and R. G. Sleight, Fluorescence assay for phospholipid membrane asymmetry. Biochemistry 1991, 30: 11819-11827.

56. Hernandez, J. M. et al., Membrane Fusion Intermediates via Directional and Full Assembly of the SNARE Complex. Science 2012, 336: 1581-1584.

57. Chan, Y.-H. M., B. van Lengerich, and S. G. Boxer, Effects of linker sequences on vesicle fusion mediated by lipid-anchored DNA oligonucleotides. Proc. Natl. Acad. Sci. USA 2009, 106: 979-984.

58. Yu, H. et al., Comparative studies of Munc18c and Munc18-1 reveal conserved and divergent mechanisms of Sec1/Munc18 proteins. Proc. Natl. Acad. Sci. USA 2013, 110: E3271-E3280.

59. Ma, C. et al., Reconstitution of the vital functions of Munc18 and Munc13 in neurotransmitter release. Science 2013, 339: 421-425. 
60. Malinin, V. S., and B. R. Lentz, Energetics of Vesicle Fusion Intermediates: Comparison of Calculations with Observed Effects of Osmotic and Curvature Stresses. Biophys. J. 2004, 86: 2951-2964.

61. Kuhlmann, J. W. 2017, Modulation of lateral membrane tension and SNARE-mediated single vesicle fusion on pore-spanning membranes, Georg-August-Universität Göttingen.

62. Ramakrishnan, S. et al., High-Throughput Monitoring of Single Vesicle Fusion Using Freestanding Membranes and Automated Analysis. Langmuir 2018, 34: 5849-5859.

63. Lira, R. B., and R. Dimova 2019, Chapter Six - Fusion assays for model membranes. A critical review. In Multiresponsive Behavior of Biomembranes and Giant Vesicles. R. Lipowsky, editor. Academic Press, pp. 229-270.

64. Kyoung, M. et al., In vitro system capable of differentiating fast Ca2+-triggered content mixing from lipid exchange for mechanistic studies of neurotransmitter release. Proc. Natl. Acad. Sci. USA 2011, 108: E304-13.

65. Yoon, T.-Y. et al., Multiple intermediates in SNARE-induced membrane fusion. Proc. Natl. Acad. Sci. USA 2006, 103: 19731-19736.

66. Kyoung, M. et al., Studying calcium-triggered vesicle fusion in a single vesicle-vesicle content and lipid-mixing system. Nat. Protoc. 2013, 8: 1-16.

67. Diao, J. et al., Synaptic proteins promote calcium-triggered fast transition from point contact to full fusion. eLife 2012, 1: e00109.

68. Gong, B. et al., High Affinity Host-Guest FRET Pair for Single-Vesicle Content-Mixing Assay. Observation of Flickering Fusion Events. J. Am. Chem. Soc. 2015, 137: 8908-8911.

69. Lai, Y. et al., Fusion pore formation and expansion induced by $\mathrm{Ca} 2+$ and synaptotagmin 1. Proc. Natl. Acad. Sci. USA 2013, 110: 1333-1338.

70. Malinin, V. S., P. Frederik, and B. R. Lentz, Osmotic and Curvature Stress Affect PEGInduced Fusion of Lipid Vesicles but Not Mixing of Their Lipids. Biophys. J. 2002, 82: 2090-2100.

71. Kreutzberger, A. J. B. et al., In vitro fusion of single synaptic and dense core vesicles reproduces key physiological properties. Nat. Commun. 2019, 10: 1-11.

72. Bowen, M. E., K. Weninger, A. T. Brunger, and S. Chu, Single molecule observation of liposome-bilayer fusion thermally induced by soluble $\mathrm{N}$-ethyl maleimide sensitive-factor attachment protein receptors (SNAREs). Biophys. J. 2004, 87: 3569-3584.

73. Liu, T. et al., SNARE-driven, 25-millisecond vesicle fusion in vitro. Biophys. J. 2005, 89: 2458-2472. 
74. Wang, T., E. A. Smith, E. R. Chapman, and J. C. Weisshaar, Lipid Mixing and Content Release in Single-Vesicle, SNARE-Driven Fusion Assay with 1-5 ms Resolution. Biophys. J. 2009, 96: 4122-4131.

75. Karatekin, E. et al., A fast, single-vesicle fusion assay mimics physiological SNARE requirements. Proc. Natl. Acad. Sci. USA 2010, 107: 3517-3521.

76. Karatekin, E., and J. E. Rothman, Fusion of single proteoliposomes with planar, cushioned bilayers in microfluidic flow cells. Nat. Protoc. 2012, 7: 903-920.

77. Domanska, M. K. et al., Single vesicle millisecond fusion kinetics reveals number of SNARE complexes optimal for fast SNARE-mediated membrane fusion. J. Biol. Chem. 2009, 284: 32158-32166.

78. Wagner, M. L., and L. K. Tamm, Reconstituted Syntaxin1A/SNAP25 Interacts with Negatively Charged Lipids as Measured by Lateral Diffusion in Planar Supported Bilayers. Biophys. J. 2001, 81: 266-275.

79. Kreutzberger, A. J. B. et al., Asymmetric Phosphatidylethanolamine Distribution Controls Fusion Pore Lifetime and Probability. Biophys. J. 2017, 113: 1912-1915.

80. Kreutzberger, A. J. B. et al., Reconstitution of calcium-mediated exocytosis of dense-core vesicles. Sci. Adv. 2017, 3: e1603208.

81. Kiessling, V., B. Liang, A. J. B. Kreutzberger, and L. K. Tamm, Planar Supported Membranes with Mobile SNARE Proteins and Quantitative Fluorescence Microscopy Assays to Study Synaptic Vesicle Fusion. Front. Mol. Neurosci. 2017, 10: 72.

82. Kreutzberger, A. J.B., V. Kiessling, and L. K. Tamm, High Cholesterol Obviates a Prolonged Hemifusion Intermediate in Fast SNARE-Mediated Membrane Fusion. Biophys. J. 2015, 109: 319-329.

83. Kocun, M., T. D. Lazzara, C. Steinem, and A. Janshoff, Preparation of Solvent-Free, Pore-Spanning Lipid Bilayers. Modeling the Low Tension of Plasma Membranes. Langmuir 2011, 27: 7672-7680.

84. Höfer, I., and C. Steinem, A membrane fusion assay based on pore-spanning lipid bilayers. Soft Matter 2011, 7: 1644.

85. Schwenen, L. L. G. et al., Resolving single membrane fusion events on planar porespanning membranes. Sci. Rep. 2015, 5: 12006.

86. Kuhlmann, J. W., M. Junius, U. Diederichsen, and C. Steinem, SNARE-Mediated SingleVesicle Fusion Events with Supported and Freestanding Lipid Membranes. Biophys. J. 2017, 112: 2348-2356. 
87. Hubrich, R. et al., SNARE-Mediated Fusion of Single Chromaffin Granules with PoreSpanning Membranes. Biophys. J. 2019, 116: 308-318.

88. Breckenridge, W. C., I. G. Morgan, J. P. Zanetta, and G. Vincendon, Adult rat brain synaptic vesicles. II. Lipid composition. Biochim. Biophys. Acta 1973, 320: 681-686.

89. Cotman, C., M. L. Blank, A. Moehl, and F. Snyder, Lipid composition of synaptic plasma membranes isolated from rat brain by zonal centrifugation. Biochemistry 1969, 8: 46064612.

90. Takamori, S. et al., Molecular anatomy of a trafficking organelle. Cell 2006, 127: 831846.

91. Schägger, H., and G. von Jagow, Tricine-sodium dodecyl sulfate-polyacrylamide gel electrophoresis for the separation of proteins in the range from 1 to $100 \mathrm{kDa}$. Anal. Biochem. 1987, 166: 368-379.

92. Schägger, H., Tricine-SDS-PAGE. Nat. Protoc. 2006, 1: 16-22.

93. Chi, Y. S. et al., Polymeric Rulers. Distance-Dependent Emission Behaviors of Fluorophores on Flat Gold Surfaces and Bioassay Platforms Using Plasmonic Fluorescence Enhancement. Adv. Funct. Mater. 2008, 18: 3395-3402.

94. Aimon, S. et al., Functional reconstitution of a voltage-gated potassium channel in giant unilamellar vesicles. PloS one 2011, 6: e25529.

95. Mühlenbrock, P. et al., Fusion Pore Formation Observed during SNARE-Mediated Vesicle Fusion with Pore-Spanning Membranes. Biophys. J. 2020, 119: 151-161.

96. Axelrod, D. et al., Mobility measurement by analysis of fluorescence photobleaching recovery kinetics. Biophys. J. 1976, 16: 1055-1069.

97. Soumpasis, D. M., Theoretical analysis of fluorescence photobleaching recovery experiments. Biophys. J. 1983, 41: 95-97.

98. Kang, M., C. A. Day, A. K. Kenworthy, and E. DiBenedetto, Simplified equation to extract diffusion coefficients from confocal FRAP data. Traffic 2012, 13: 1589-1600.

99. Schneider, G. et al., Distance-Dependent Fluorescence Quenching on Gold Nanoparticles Ensheathed with Layer-by-Layer Assembled Polyelectrolytes. Nano Lett. 2006, 6: 530536.

100. Gordon, G. W., B. Chazotte, X. F. Wang, and B. Herman, Analysis of simulated and experimental fluorescence recovery after photobleaching. Data for two diffusing components. Biophys. J. 1995, 68: 766-778. 
101. Jönsson, P., M. P. Jonsson, J. O. Tegenfeldt, and F. Höök, A method improving the accuracy of fluorescence recovery after photobleaching analysis. Biophys. J. 2008, 95: $5334-5348$.

102. Gleisner, M. 2016, Interaction of Epsin N-Terminal Homology domain (ENTH) with artificial lipid membranes as a function of lateral tension, Georg-August-Universität Göttingen.

103. Sbalzarini, I. F., and P. Koumoutsakos, Feature point tracking and trajectory analysis for video imaging in cell biology. J. Struct. Biol. 2005, 151: 182-195.

104. Komorowski, K. et al., Vesicle Adhesion and Fusion Studied by Small-Angle X-Ray Scattering. Biophys. J. 2018, 114: 1908-1920.

105. Kiessling, V. et al., Rapid fusion of synaptic vesicles with reconstituted target SNARE membranes. Biophys. J. 2013, 104: 1950-1958.

106. Chen, R. F., and J. R. Knutson, Mechanism of fluorescence concentration quenching of carboxyfluorescein in liposomes. Energy transfer to nonfluorescent dimers. Anal. Biochem. 1988, 172: 61-77.

107. Nagle, J. F., and S. Tristram-Nagle, Structure of lipid bilayers. Biochim. Biophys. Acta, Rev. Biomembr. 2000, 1469: 159-195.

108. Jahn, R., and R. H. Scheller, SNAREs--engines for membrane fusion. Nat. Rev. Mol. Cell Biol. 2006, 7: 631-643.

109. Chiang, H.-C. et al., Post-fusion structural changes and their roles in exocytosis and endocytosis of dense-core vesicles. Nat. Commun. 2014, 5: 3356.

110. Staal, R. G. W., E. V. Mosharov, and D. Sulzer, Dopamine neurons release transmitter via a flickering fusion pore. Nat. Neurosci. 2004, 7: 341-346.

111. Fulop, T., S. Radabaugh, and C. Smith, Activity-dependent differential transmitter release in mouse adrenal chromaffin cells. J. Neurosci. 2005, 25: 7324-7332.

112. Klyachko, V. A., and M. B. Jackson, Capacitance steps and fusion pores of small and large-dense-core vesicles in nerve terminals. Nature 2002, 418: 89-92.

113. Zhou, Z., S. Misler, and R. H. Chow, Rapid fluctuations in transmitter release from single vesicles in bovine adrenal chromaffin cells. Biophys. J. 1996, 70: 1543-1552.

114. Toledo, G. A. d., R. Fernández-Chacón, and J. M. Fernández, Release of secretory products during transient vesicle fusion. Nature 1993, 363: 554-558.

115. Witkowska, A., and R. Jahn, Rapid SNARE-Mediated Fusion of Liposomes and Chromaffin Granules with Giant Unilamellar Vesicles. Biophys. J. 2017, 113: 1251-1259. 
116. Witkowska, A., L. Jablonski, and R. Jahn, A convenient protocol for generating giant unilamellar vesicles containing SNARE proteins using electroformation. Sci. Rep. 2018, 8: 9422.

117. Girard, P. et al., A new method for the reconstitution of membrane proteins into giant unilamellar vesicles. Biophys. J. 2004, 87: 419-429.

118. Garten, M., S. Aimon, P. Bassereau, and G. E. S. Toombes, Reconstitution of a Transmembrane Protein, the Voltage-gated Ion Channel, KvAP, into Giant Unilamellar Vesicles for Microscopy and Patch Clamp Studies. JoVE 2015: 52281.

119. Jørgensen, I. L., G. C. Kemmer, and T. G. Pomorski, Membrane protein reconstitution into giant unilamellar vesicles: a review on current techniques. Eur. Biophys. J. 2017, 46: $103-119$.

120. Doeven, M. K. et al., Distribution, lateral mobility and function of membrane proteins incorporated into giant unilamellar vesicles. Biophys. J. 2005, 88: 1134-1142.

121. J C Lee, and S N Timasheff, The stabilization of proteins by sucrose. J. Biol. Chem. 1981, 256: 7193-7201.

122. Hincha, D. K., E. Zuther, and A. G. Heyer, The preservation of liposomes by raffinose family oligosaccharides during drying is mediated by effects on fusion and lipid phase transitions. Biochim. Biophys. Acta, Rev. Biomembr. 2003, 1612: 172-177.

123. Battle, A. R., E. Petrov, P. Pal, and B. Martinac, Rapid and improved reconstitution of bacterial mechanosensitive ion channel proteins $\mathrm{MscS}$ and $\mathrm{MscL}$ into liposomes using a modified sucrose method. FEBS Lett. 2009, 583: 407-412.

124. Kreir, M. et al., Rapid screening of membrane protein activity: electrophysiological analysis of OmpF reconstituted in proteoliposomes. Lab Chip 2008, 8: 587-595.

125. Varnier, A. et al., A simple method for the reconstitution of membrane proteins into giant unilamellar vesicles. J. Membr. Biol. 2010, 233: 85-92.

126. Galkin, M. A. et al., Detergent-free Ultrafast Reconstitution of Membrane Proteins into Lipid Bilayers Using Fusogenic Complementary-charged Proteoliposomes. J. Vis. Exp. (Journal of Visualized Experiments Abbreviated) 2018.

127. Ishmukhametov, R. R., A. N. Russell, and R. M. Berry, A modular platform for onestep assembly of multi-component membrane systems by fusion of charged proteoliposomes. Nat. Commun. 2016, 7: 13025.

128. Walde, P., K. Cosentino, H. Engel, and P. Stano, Giant vesicles: preparations and applications. ChemBioChem 2010, 11: 848-865. 
129. Göpfrich, K. et al., One-Pot Assembly of Complex Giant Unilamellar Vesicle-Based Synthetic Cells. ACS Synth. Biol. 2019, 8: 937-947.

130. Weiss, M. et al., Sequential bottom-up assembly of mechanically stabilized synthetic cells by microfluidics. Nat. Mater. 2018, 17: 89-96.

131. Kliesch, T.-T. et al., Membrane tension increases fusion efficiency of model membranes in the presence of SNAREs. Sci. Rep., 7: 1-13.

132. Sieber, J. J. et al., Anatomy and dynamics of a supramolecular membrane protein cluster. Science 2007, 317: 1072-1076.

133. Knowles, M. K. et al., Single secretory granules of live cells recruit syntaxin-1 and synaptosomal associated protein 25 (SNAP-25) in large copy numbers. Proc. Natl. Acad. Sci. USA 2010, 107: 20810-20815.

134. Bacia, K. et al., SNAREs prefer liquid-disordered over "raft" (liquid-ordered) domains when reconstituted into giant unilamellar vesicles. J. Biol. Chem. 2004, 279: 37951-37955.

135. Guo, L. et al., Molecular diffusion measurement in lipid bilayers over wide concentration ranges: a comparative study. ChemPhysChem 2008, 9: 721-728.

136. Carrer, D. C., A. W. Schmidt, H.-J. Knölker, and P. Schwille, Membrane DomainDisrupting Effects of 4-Substitued Cholesterol Derivatives. Langmuir 2008, 24: 88078812.

137. Schneider, F. et al., Diffusion of lipids and GPI-anchored proteins in actin-free plasma membrane vesicles measured by STED-FCS. Mol. Biol. Cell 2017, 28: 1507-1518.

138. Ramadurai, S. et al., Lateral diffusion of membrane proteins. J. Am. Chem. Soc. 2009, 131: 12650-12656.

139. Ramakrishnan, S. et al., Synaptotagmin oligomers are necessary and can be sufficient to form a Ca2+-sensitive fusion clamp. FEBS Lett. 2019, 593: 154-162.

140. Przybylo, M. et al., Lipid diffusion in giant unilamellar vesicles is more than 2 times faster than in supported phospholipid bilayers under identical conditions. Langmuir 2006, 22: 9096-9099.

141. Machán, R., and M. Hof, Lipid diffusion in planar membranes investigated by fluorescence correlation spectroscopy. Biochim. Biophys. Acta 2010, 1798: 1377-1391.

142. Zhang, L., and S. Granick, Lipid diffusion compared in outer and inner leaflets of planar supported bilayers. J. Chem. Phys. 2005, 123: 211104.

143. Honigmann, A. et al., Phosphatidylinositol 4,5-bisphosphate clusters act as molecular beacons for vesicle recruitment. Nat. Struct. Biol. 2013, 20: 679-686. 
144. Saffman, P. G., and M. Delbrück, Brownian motion in biological membranes. Proc. Natl. Acad. Sci. USA 1975, 72: 3111-3113.

145. Ribrault, C. et al., Syntaxin1A lateral diffusion reveals transient and local SNARE interactions. J. Neurosci. 2011, 31: 17590-17602.

146. Vaz, W. L.C., F. Goodsaid-Zalduondo, and K. Jacobson, Lateral diffusion of lipids and proteins in bilayer membranes. FEBS Lett. 1984, 174: 199-207.

147. Murray, D. H., and L. K. Tamm, Molecular mechanism of cholesterol- and polyphosphoinositide-mediated syntaxin clustering. Biochemistry 2011, 50: 9014-9022.

148. Sonnleitner, A., G. J. Schütz, and T. Schmidt, Free Brownian Motion of Individual Lipid Molecules in Biomembranes. Biophys. J. 1999, 77: 2638-2642.

149. Hubrich, R. 2018, Pore-spanning membranes - a versatile tool to analyze SNAREmediated single vesicle fusion, Georg-August-Universität Göttingen.

150. Jing, H., S. Sinha, H. S. Sachar, and S. Das, Interactions of gold and silica nanoparticles with plasma membranes get distinguished by the van der Waals forces: Implications for drug delivery, imaging, and theranostics. Colloids Surf., B 2019, 177 : 433-439.

151. Laage, R., J. Rohde, B. Brosig, and D. Langosch, A conserved membrane-spanning amino acid motif drives homomeric and supports heteromeric assembly of presynaptic SNARE proteins. J. Biol. Chem. 2000, 275: 17481-17487.

152. Sharma, S., and M. Lindau, t-SNARE Transmembrane Domain Clustering Modulates Lipid Organization and Membrane Curvature. J. Am. Chem. Soc. 2017, 139: 18440-18443.

153. Sieber, J. J. et al., The SNARE motif is essential for the formation of syntaxin clusters in the plasma membrane. Biophys. J. 2006, 90: 2843-2851.

154. Lang, T. et al., SNAREs are concentrated in cholesterol-dependent clusters that define docking and fusion sites for exocytosis. EMBO J. 2001, 20: 2202-2213.

155. Kiessling, V., B. Liang, and L. K. Tamm, Reconstituting SNARE-mediated membrane fusion at the single liposome level. Methods Cell Biol. 2015, 128: 339-363.

156. Mohrmann, R. et al., Fast vesicle fusion in living cells requires at least three SNARE complexes. Science 2010, 330: 502-505.

157. van den Bogaart, G., and R. Jahn, Counting the SNAREs needed for membrane fusion. J. Mol. Cell Biol. 2011, 3: 204-205.

158. Yavuz, H. et al., Arrest of trans-SNARE zippering uncovers loosely and tightly docked intermediates in membrane fusion. J. Biol. Chem. 2018, 293: 8645-8655. 
159. Böcker, M. et al., Imaging and Patterning of Pore-Suspending Membranes with Scanning Ion Conductance Microscopy. Langmuir 2009, 25: 3022-3028.

160. Schütte, O. M. et al., Size and Mobility of Lipid Domains Tuned by Geometrical Constraints. Proc. Natl. Acad. Sci. USA 2017, 114.

161. Raccis, R. et al., Confined diffusion in periodic porous nanostructures. ACS Nano 2011, 5: 4607-4616.

162. Jordan, R., E. A. Lemke, and J. Klingauf, Visualization of synaptic vesicle movement in intact synaptic boutons using fluorescence fluctuation spectroscopy. Biophys. J. 2005, 89: 2091-2102.

163. Domanska, M. K., V. Kiessling, and L. K. Tamm, Docking and fast fusion of synaptobrevin vesicles depends on the lipid compositions of the vesicle and the acceptor SNARE complex-containing target membrane. Biophys. J. 2010, 99: 2936-2946.

164. Ji, H. et al., Protein determinants of SNARE-mediated lipid mixing. Biophys. J. 2010, 99: 553-560.

165. Wölfel, M., and R. Schneggenburger, Presynaptic Capacitance Measurements and Ca 2+ Uncaging Reveal Submillisecond Exocytosis Kinetics and Characterize the $\mathrm{Ca} 2+$ Sensitivity of Vesicle Pool Depletion at a Fast CNS Synapse. J. Neurosci. 2003, 23: 7059 7068.

166. Cypionka, A. 2009, Neue Einblicke in die SNARE-vermittelte Fusion: Detektion einzelner Proteoliposomen mit einem konfokalen Mikroskop, Georg-August-Universität Göttingen.

167. Stein, A. 2008, Untersuchungen zur Rekonstitution der SNARE-vermittelten Membranfusion. Freie Universität Berlin.

168. Floyd, D. L. et al., Single-particle kinetics of influenza virus membrane fusion. Proc. Natl. Acad. Sci. USA 2008, 105: 15382-15387.

169. Sharma, S., and M. Lindau, The fusion pore, 60 years after the first cartoon. FEBS Lett. 2018, 592: 3542-3562.

170. Spruce, A. E., L. J. Breckenridge, A. K. Lee, and W. Almers, Properties of the fusion pore that forms during exocytosis of a mast cell secretory vesicle. Neuron 1990, 4: 643654.

171. Patty, P. J., and B. J. Frisken, The Pressure-Dependence of the Size of Extruded Vesicles. Biophys. J. 2003, 85: 996-1004.

172. Lu, X., F. Zhang, J. A. McNew, and Y.-K. Shin, Membrane fusion induced by neuronal SNAREs transits through hemifusion. J. Biol. Chem. 2005, 280: 30538-30541. 
173. Chen, Y. A., and R. H. Scheller, SNARE-mediated membrane fusion. Nat. Rev. Mol. Cell Biol. 2001, 2: 98-106.

174. Brunger, A. T., K. Weninger, M. Bowen, and S. Chu, Single-molecule studies of the neuronal SNARE fusion machinery. Annu. Rev. Biochem. 2009, 78: 903-928.

175. Yang, Y. et al., Dissection of SNARE-driven membrane fusion and neuroexocytosis by wedging small hydrophobic molecules into the SNARE zipper. Proc. Natl. Acad. Sci. USA 2010, 107: 22145-22150.

176. Liu, T., T. Wang, E. R. Chapman, and J. C. Weisshaar, Productive hemifusion intermediates in fast vesicle fusion driven by neuronal SNAREs. Biophys. J. 2008, 94: $1303-1314$.

177. Zhao, W.-D. et al., Hemi-fused structure mediates and controls fusion and fission in live cells. Nature 2016, 534: 548-552.

178. Shin, W. et al., Visualization of Membrane Pore in Live Cells Reveals a DynamicPore Theory Governing Fusion and Endocytosis. Cell 2018, 173: 934-945.e12.

179. Wen, P. J. et al., Actin dynamics provides membrane tension to merge fusing vesicles into the plasma membrane. Nat. Commun. 2016, 7: 1-14.

180. Anantharam, A. et al., Localized topological changes of the plasma membrane upon exocytosis visualized by polarized TIRFM. J. Cell Biol. 2010, 188: 415-428.

181. Gandhi, S. P., and C. F. Stevens, Three modes of synaptic vesicular recycling revealed by single-vesicle imaging. Nature 2003, 423: 607-613.

182. Sankaranarayanan, S., and T. A. Ryan, Real-time measurements of vesicle-SNARE recycling in synapses of the central nervous system. Nat. Cell Biol. 2000, 2: 197-204.

183. Aravanis, A. M., J. L. Pyle, and R. W. Tsien, Single synaptic vesicles fusing transiently and successively without loss of identity. Nature 2003, 423: 643-647.

184. Park, Y. et al., Controlling synaptotagmin activity by electrostatic screening. Nat. Struct. Mol. Biol. 2012, 19: 991-997. 


\section{Curriculum vitae}

\section{Personal data}

Name: $\quad$ Peter Mühlenbrock

Date of birth: $\quad 14.11 .1992$

Place of birth: Göttingen, Germany

Citizenship: German

\section{Education}

Since 2017 PhD thesis at the Georg-August University Göttingen (Prof. Dr. Claudia Steinem), title of the thesis: "SNARE-mediated membrane fusion on porespanning membranes - several fusion pathways analyzed by single-vesicle content release".

2014-2016 Graduate studies in chemistry at the Georg-August University Göttingen, degree: Master of Science, title of the Master thesis: "Investigating the mechanical properties of F-actin bound pore-spanning lipid bilayers by means of atomic force microscopy".

2011-2014 Undergraduate studies in chemistry at the Georg-August University Göttingen, degree: Bachelor of Science, title of the Bachelor thesis: "Synthesis and DNA binding studies of a platinum complex/peptide chimera mimicking IHF".

2009-2011 Allgemeine Hochschulreife at Gymnasium Corvinianum Northeim 


\section{Scientific contributions}

\section{Publications}

2020 P. Mühlenbrock, K. Herwig, L. Vuong, I. Mey and C. Steinem Biophys. J. 2020, 119: 151-161.

2020 H. Hubrich, I. Mey, B. R. Brückner, P, Mühlenbrock, S. Nehls, L. Grabenhorst, T. Oswald, C. Steinem, A. Jahnshof Nano Lett. 2020, 20: 6329-6335.

\section{Selected oral presentations}

International Meeting of the DGfB Membrane Section, Several fusion pathways: SNARE-mediated membrane fusion with PSMs visualized by single-vesicle content release.

2019 SFB 803 winter school, Pore-spanning membranes: a tool to study single vesicle content release in SNARE-mediated membrane fusion.

SFB 803 autumn school, SNARE-mediated membrane fusion on a single vesicle level.

\section{Selected poster presentations}

International Meeting of the DGfB Membrane Section, Several Fusion Pathways: SNARE-Mediated Membrane Fusion with Pore-Spanning Membranes Visualized by Single-Vesicle Content Release.

Biennial Meeting of the German Biophysical Society, Pore-spanning membranes: a tool to study single vesicle content release in SNAREmediated membrane fusion.

$19^{\text {th }}$ IUPAB and $11^{\text {th }}$ EBSA congress, Pore-spanning membranes: a tool to study single vesicle content release in SNARE-mediated membrane fusion. 


\section{Danksagung}

An dieser Stelle möchte ich mich herzlichst bei Prof. Dr. Claudia Steinem für die kompetente Betreuung meiner Doktorarbeit und stets hilfreiche wissenschaftliche Diskussionen bedanken.

Als Mitglieder meines Prüfungskomitees gilt mein Dank zudem Prof. Dr. Tim Salditt und Prof. Dr. Silvio O. Rizzoli für Ihr konstruktives Feedback und hilfreichen Diskurs während meiner Arbeit.

Mein spezieller Dank richtet sich an Ingo. Nicht nur hattest du immer ein offenes Ohr für mich, sondern halfst mir meine teilweise wirren Ideen in Matlab-Worte zu fassen, welche Ordnung in den Datendschungel brachten. Auch Jerry und Johannes gilt hier mein Dank, die mir stets bei kleineren Probleme halfen, deren Ursprung wohl oftmals vor dem Rechner saß.

Ich danke meinen Bachelorstudentinnen Loan, Lara, und Kira für die tatkräftige und motivierte Untersuchung ihrer Themen. Eure Betreuung hat mir sehr viel Spaß gemacht und ich hoffe, ihr habt Erfolg auf eurem weiteren Weg.

Danke an Jutta und Micha für Ihre große Hilfe bei meinen alltäglichen Laborarbeiten, denn nur durch euch war das reibungslose Arbeiten im Labor möglich. Danke hier auch an Raphael und Jan für das Einarbeiten in das Thema dieser Arbeit und nachfolgende hilfreiche Tipps.

Ich möchte zudem unseren Sekretärinnen Dana, Marianne und Melanie danken. Nicht nur konnte man sicher sein, bei euch Süßigkeiten und einen kurzen Plausch zu finden, ohne euch hätte ich wohl auch nie einen Dienstreiseantrag fehlerfrei ausgefüllt.

Vielen Dank an Dominik, Yeimar, Tim, Niko, Philipp, und Kristina für das Korrekturlesen dieser Arbeit und eure hilfreichen Anregungen. Ein besonderer Dank geht hier an Nils und Ingo, ihr habt echt den Löwenanteil übernommen.

Dominik und Jutta gilt mein Dank für das Kümmern um die Vielzahl meiner Pflanzen während meiner Abwesenheit. Vor allem die letzten Monate hätten sie ohne eure Hilfe nicht überlebt.

Dem gesamten AK danke ich für die schönen Jahre. Mit euch konnte man nicht nur gute Zeiten teilen, ihr habt einen auch durch schlechte Zeiten gebracht. Ich werde unsere eloquenten Diskussionen im Kaffeeraum, die hitzigen Kicker-Duelle und gemütlichen Grillabende sehr vermissen.

Und zu guter Letzt Danke! an meine Familie, meine Freunde, und Laura für die Unterstützung während der letzten Jahre. Ich freue mich, euch auch für die kommenden Jahre an meiner Seite zu wissen. 